\title{
Stable Isotopes of \\ Platinum: Measurement and Application to \\ Constraining the Formation and Differentiation of Earth
}

by

John Benjamin Creech

A thesis

submitted to the Victoria University of Wellington

in fulfilment of the

requirements for the degree of

Doctor of Philosophy

in Geology.

Victoria University of Wellington

2014 


\begin{abstract}
A wide range of novel, non-traditional, stable isotope systems have been developed over the last decade, largely as a result of the advent of multiplecollector inductively coupled plasma mass spectrometry (MC-ICPMS), and continue to provide valuable new insights in the earth, environmental and planetary sciences. The platinum (Pt) stable isotope system represents a potentially powerful but, as yet, unexplored addition to this suite of stable isotope tracers. Pt has six naturally occurring isotopes, and can exist in a range of oxidation states. The geochemical behaviour of Pt coupled with the relatively large mass difference (ca. $2 \%$ ) between the abundant heavy and light isotopes and its variable oxidation states leads to potential applications in tracing a range of natural processes. In particular, the strong elemental partitioning of $\mathrm{Pt}$ between metals and silicates makes the Pt stable isotope system uniquely suited to tracing processes of Earth's accretion and differentiation. This study aims to develop new techniques for measurement of $\mathrm{Pt}$ stable isotopes in geological samples, and to apply these to terrestrial and meteorite samples to attempt to resolve outstanding questions relating to Earth's early evolution.

A technique was developed for measurement of $\mathrm{Pt}$ stable isotope ratios using multiple collector inductively coupled plasma mass spectrometry (MCICPMS), employing a ${ }^{196} \mathrm{Pt}-{ }^{198} \mathrm{Pt}$ double-spike to correct for instrumental mass fractionation. Results are reported in terms of $\delta^{198} \mathrm{Pt}$, which represents the per mil difference in the ${ }^{198} \mathrm{Pt} /{ }^{194} \mathrm{Pt}$ ratio from the IRMM-010 Pt isotope standard. A range of analytical tests were conducted and show that this approach has a reproducibility of ca. $\pm 0.04 \%$ on $\delta^{198} \mathrm{Pt}$ (i.e., $\pm 0.01 \%$ o $\mathrm{amu}^{-1}$ ) for Pt solution standards, and is insensitive to minor amounts of matrix that may be retained after chemical purification of Pt. Measurements of
\end{abstract}


Pt solution standards conducted using two different MC-ICPMS instruments showed resolvable variations, which suggest that natural fractionations exist that exceed the reproducibility of the technique.

Techniques were also developed for dissolution of natural samples and chemical separation of Pt. Geological standards were digested using a nickel sulphide fire assay technique, which pre-concentrates the highly siderophile elements in a NiS bead that is readily dissolved in acid. This was followed by chemical separation of Pt from digested samples using anion exchange chemical techniques. Elution curves were constructed for a range of synthetic rock matrices. These tests show that Pt separation is achieved with $>90 \%$ Pt yield and ca. 95\% purity. Analytical tests show that this level of $\mathrm{Pt}$ separation is sufficient for accurate determination of Pt stable isotope ratios by double-spike MC-ICPMS. These techniques were then applied to 11 international geological standard reference materials representing mantle peridotites, igneous samples, and Pt ore materials. The reproducibility in natural samples was determined by processing multiple replicate digestions of a standard reference material, and was shown to be ca. $\pm 0.08 \%$ o (2 sd). Pt stable isotope data for the full set of reference materials have a range of $\delta^{198} \mathrm{Pt}$ values with offsets of up to $0.40 \%$ o from the IRMM-010 standard, which are readily resolved with this technique. Mantle samples yielded the lightest (most negative) isotopic compositions of the terrestrial standards, with igneous and Pt ore samples defining a continuous trend towards zero, which is consistent with the IRMM-010 standard being derived from a Pt ore. These results demonstrate the potential of the Pt isotope system as a tracer in geochemical systems.

The techniques developed above were then applied to investigate an outstanding problem relating to Earth's accretion and differentiation. Highly siderophile elements (HSE) are strongly partitioned into the cores of terrestrial planets during core formation, and the abundances of HSE in Earth's mantle compared with primitive meteorites have provided key constraints on models of Earth's early evolution. Two leading models to explain the HSE 
abundances in the silicate Earth involve either a late-veneer of chondritic material that was added after core formation or core formation in a deep magma ocean. The platinum (Pt) stable isotope system represents a novel tool for investigating these processes. Using the techniques developed above, Pt stable isotope ratios were measured in a range of meteorite samples, including enstatite, ordinary and carbonaceous chondrites, primitive achondrites, achondrites and iron meteorites, as well as additional terrestrial mantle xenolith samples. Our data set reveals that the Pt stable isotopic composition of Earth's mantle overlaps with all of the chondrite groups. Primitive achondrite and ureilite samples revealed the heaviest compositions of all meteorite groups. These data suggest that metal-silicate differentiation produces an isotopic fractionation for $\mathrm{Pt}$, with heavy isotopes being preferentially retained in the silicate phase. Thus, Earth's mantle is expected to have been significantly enriched in the heavy isotopes of $\mathrm{Pt}$ during core formation, even if metal-silicate differentiation took place in a magma ocean. The absence of a large fractionation between chondrites, representing the composition of the undifferentiated Earth, and the mantle suggests that the signature of core formation in the mantle has been subsequently overprinted. Considering the overlap between the Pt stable isotopic compositions of the mantle and chondrites, the most likely means for overprinting the composition of the mantle is by addition of a chondritic late-veneer. Mixing calculations show that addition of $0.5 \%$ of Earth's mass by a late-veneer of chondritic material would be sufficient to overprint highly fractionated Pt stable isotope signatures resulting from core-formation. 


\section{Acknowledgments}

Firstly, I must acknowledge my supervisors, Joel Baker, Monica Handler and Martin Bizzarro. Thank you for the opportunity to work on this interesting project, which has allowed me to explore my interests spanning your combined range of expertise. My thanks to Joel, for all of your input and your patient guidance, and for staying involved with my research even when it would have been easier not to. At the end of it all, I have learned a great deal from your experience and insight. To Monica, I am grateful for all of your guidance and support, and for the opportunities to travel to present my work overseas and spend some time working in another laboratory during my PhD. Thank you to Martin for the opportunity to work with you in Denmark, which was a valuable experience for me and also improved the results of parts of this thesis. I would also like to acknowledge Martin Schiller, who has long been one of my best friends, and who I enjoyed having the opportinity to work with during my $\mathrm{PhD}$.

I would like to thank my sources of funding during my $\mathrm{PhD}$ : Victoria University for providing me with a PhD Scholarship which provided my living costs, the VUW post-graduate students association for the PGSA Research Excellence Award which funded my trip to a workshop in Hawaii, SGEES for the Paul Vella Research Excellence Award, and the Royal Society of New Zealand Marsden Fund for funding the wider Pt project which paid for part of my living costs and all of my argon, acids, conference trips, etc. that I could not have done all of this without.

Thank you to Sabrina, our lab technician, for meeting my various de- 
mands for equipment and materials, for sharing my frustration when things didn't happen the way they ought to, and for the effort you put in to your work. Thanks also to Prof. John Spencer from SCPS and Manu and Alan at the SCPS workshop for their willingness to help out with various unusual things. Thanks to Chad Paton and Bence Paul for their work developing Iolite and willingness to help others, and particularly to Bence for helping me to develop a new tool that saved me (and, now, others too) a huge amount of time and difficulty.

I have been lucky to have made some great friends during my years at VUW, amongst fellow students (past and present) and amongst the academic staff. You have been like a big extended family to us and have made my time here much more enjoyable. I also want to acknowledge the support of my non-uni friends, who I don't see as much of as I'd like, but have still somehow not forgotten me. I'm sorry to not name you individually (to do so would start to rival some of the chapters of this thesis), but I am sure you know who you are, and that I am truly grateful for your friendship.

Finally, thank you to my family. Jess, thank you for your incredible patience all the times twenty minutes turned into three (or five, or ten) hours, for being an amazing mum to Jackson, and for the unconditional love and support that you give me. To my parents, who never doubted I could do this and have supported me in every way. To Mum, for going way above and beyond what anybody could hope for, to the point of travelling around the world (twice now!) so I wouldn't have to be apart from Jackson. To Dad, for teaching me to always work hard and always giving me encouragement. To my brothers, Nick and Jake, for doing such interesting and different things that inspire me and also give me fun places to visit. And last, but not least, to Jackson, my bestest buddy who is so clever and fun. You have always given me a breath of fresh air and and a handhold on reality when I needed it, and you never failed to brighten my day. You are all amazing people and you been so good to me. I dedicate this to you. 


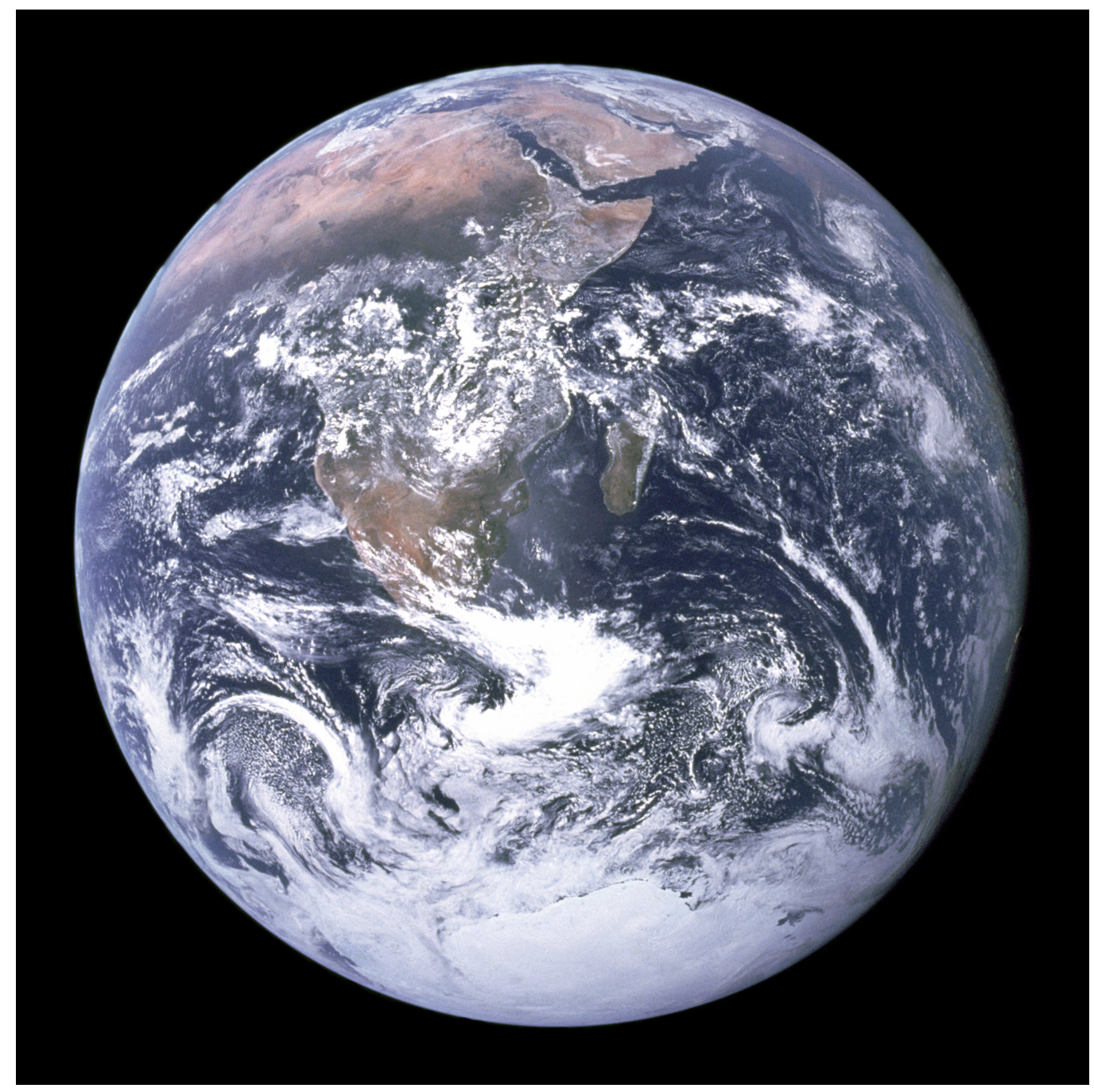

Earth, as photographed from Apollo 17. (Image credit: NASA). 


\section{Contents}

$\begin{array}{lll}1 & \text { Introduction } & 1\end{array}$

1.1 Objectives of this thesis . . . . . . . . . . . . . 1

1.2 Geochemistry of platinum and the platinum group elements . 3

1.2 .1 The mantle . . . . . . . . . . . . . 3

1.2 .2 Igneous systems . . . . . . . . . . . . . . 5

1.2 .3 Platinum ores . . . . . . . . . . . . . . . . . . . 7

1.2 .4 Marine environment . . . . . . . . . . . . . . 8

1.2 .5 Hydrothermal systems . . . . . . . . . . . . 8

1.2 .6 Meteorites . . . . . . . . . . . . . . . . . . . . 9

1.3 Terrestrial planet formation and evolution . . . . . . . . . . . 10

1.3.1 Solar System and planet formation . . . . . . . . . . . 10

1.3.2 Accretion and differentiation of terrestrial planets . . . 11

1.3.3 Models for Earth's accretion and differentiation . . . . 14

1.4 Meteorite samples . . . . . . . . . . . . . . . . . . 23

1.4 .1 Classification of Meteorites . . . . . . . . . . . . . . 24

1.4 .2 Chondrites . . . . . . . . . . . . . . . . . 26

1.4 .3 Non-chondrites . . . . . . . . . . . . . . 32

1.5 Non-traditional stable isotope systems . . . . . . . . . . . . . 38

1.5 .1 Iron stable isotopes . . . . . . . . . . . . . . . 40

1.5 .2 Silicon stable isotopes . . . . . . . . . . . . . 43

1.5 .3 Chromium stable isotopes . . . . . . . . . . . . . 46

1.5 .4 Nickel stable isotopes . . . . . . . . . . . . . . . . . 47 
1.5 .5 Platinum stable isotopes . . . . . . . . . . . . 51

1.6 Thesis structure . . . . . . . . . . . . . . . . . . . . . . 55

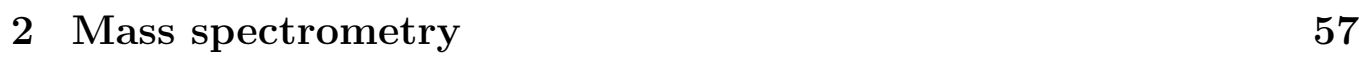

2.1 Introduction . . . . . . . . . . . . . . . . . . . . . . . 58

2.2 Double-spike methodology . . . . . . . . . . . . . . . . . . . 61

$2.2 .1 \quad$ Pt double-spike design . . . . . . . . . . . . . . . . 63

2.3 Materials and methods . . . . . . . . . . . . . . . . . . . . . . 64

2.3 .1 Chemicals and standard solutions . . . . . . . . . . 64

2.3 .2 Preparation of platinum double-spikes . . . . . . . . . 65

$2.3 .3 \quad$ Mass spectrometry $\ldots \ldots \ldots 6$

2.3 .4 Interference correction . . . . . . . . . . . 70

2.3 .5 Data reduction . . . . . . . . . . . . . 70

2.3 .6 Reporting of platinum stable isotope data . . . . . . . 71

2.3 .7 Analytical tests . . . . . . . . . . . . . . 71

2.4 Results and Discussion . . . . . . . . . . . . . . . . . . . . . . 73

2.4 .1 Platinum isotopic composition of the IRMM-010 standard . . . . . . . . . . . . . . . 73

2.4 .2 Calibration of the platinum double-spikes . . . . . . 77

2.4 .3 Internal precision and external reproducibility of double-spike-corrected platinum stable isotope ratios . 79

$2.4 .4 \quad$ Analytical tests . . . . . . . . . . . . . . . 83

2.5 Conclusions . . . . . . . . . . . . . . . . . . . . . . . . . . 87

$\begin{array}{lll}3 & \text { Chemical separation of } \mathrm{Pt} & 90\end{array}$

3.1 Introduction . . . . . . . . . . . . . . . . . . . . . . . . . . 91

3.2 Samples . . . . . . . . . . . . . . . . . . . . . . . . . . . . 92

3.3 Materials and methods . . . . . . . . . . . . . . . . . . . . . . 95

3.3.1 Reagents, standard solutions and spikes . . . . . . . . . 95

3.3 .2 Laboratory environment . . . . . . . . . . . . . . . . . 96

3.3 .3 Sample digestion . . . . . . . . . . . . . . . . . . . 97

3.3.4 Anion-exchange chemistry . . . . . . . . . . . . . . . . 99 
3.3.5 Column calibration by ICP-MS . . . . . . . . . . . . 101

3.3.6 Platinum stable isotope analysis by double-spike MCICPMS . . . . . . . . . . . . . . . . . . . 101

3.4 Results and Discussion . . . . . . . . . . . . . . . . . . . 103

3.4.1 Chemical separation and recovery of $\mathrm{Pt}$. . . . . . . . . 103

3.4 .2 Pt concentrations of standards . . . . . . . . . . . . . 106

3.4.3 Platinum stable isotope results . . . . . . . . . . . 107

3.5 Conclusions . . . . . . . . . . . . . . . . . . . . . . . . . . . 113

4 Terrestrial and extraterrestrial results 118

4.1 Introduction . . . . . . . . . . . . . . . . . . . . . . . 120

4.2 Samples and methods . . . . . . . . . . . . . . . . . . . . . 123

$4.2 .1 \quad$ Terrestrial and meteorite samples . . . . . . . . . . . 123

4.2 .2 Analytical methods . . . . . . . . . . . . . . . . . . . 124

4.3 Results . . . . . . . . . . . . . . . . . . . . . . . . . . . . . 126

4.4 Discussion . . . . . . . . . . . . . . . . . . . . . . . . . 129

4.4.1 The origin of Pt stable isotope variations within meteorite groups . . . . . . . . . . . . . . . . . . 129

$4.4 .2 \quad$ Pt stable isotope composition of Earth's mantle . . . . 131

4.4 .3 Implications of Pt stable isotopes for accretion models 132

4.5 Conclusions . . . . . . . . . . . . . . . . . . . 139

$\begin{array}{lll}5 & \text { Conclusions } & 141\end{array}$

5.1 Techniques for platinum stable isotope measurements . . . . . 142

5.1.1 Precise and accurate measurement of platinum stable isotope ratios . . . . . . . . . . . . . . . . . . 143

5.1 .2 Purification of platinum from natural samples . . . . . 144

5.2 Platinum stable isotopes in terrestrial and meteorite samples and implications for accretion and differentiation models . . . 145

$5.2 .1 \quad$ Platinum stable isotopes in terrestrial samples . . . . . 145

5.2 .2 Platinum stable isotopes in meteorites . . . . . . . . . 146 
5.2.3 Platinum stable isotope implications for accretion and differentiation models . . . . . . . . . . . . . . . . . . . 147

5.3 Suggestions for future work . . . . . . . . . . . . . . . . . . . 148

$\begin{array}{ll}\text { Combined References } & 151\end{array}$

\begin{tabular}{ll}
\hline Appendices & 185
\end{tabular}

\begin{tabular}{ll}
\hline A Samples & 186
\end{tabular}

B Reprint of JAAS paper 193

C Reprint of Chemical Geology paper 207

D Preprint of GGR paper (IsoSpike) 216 


\section{List of Figures}

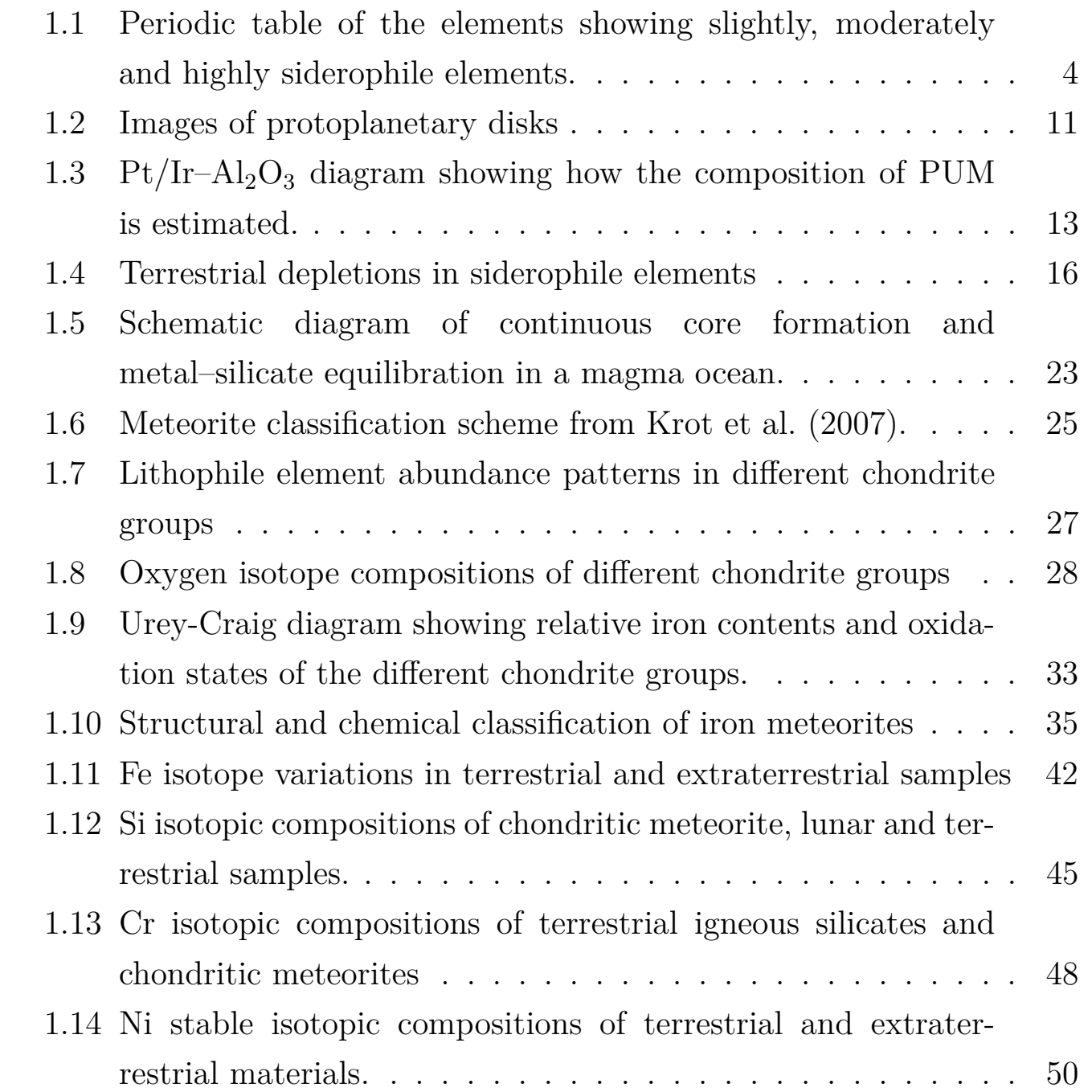


List of Figures $\quad$ ix

$2.1 \quad$ Isotopic composition of natural Pt / IRMM-010 . . . . . . . . 72

$2.2 \quad$ Double spike calibration mixtures . . . . . . . . . . . . . . 73

2.3 Reproducibility of DS-corrected MC-ICPMS measurements . . 76

2.4 Pt stable isotope variations in laboratory standards . . . . . . 80

2.5 Results of element doping tests . . . . . . . . . . . . . . 83

$2.6 \quad$ Results of low intensity tests and peak position tests . . . . . 87

$3.1 \quad$ Flow chart illustrating the Pt separation scheme . . . . . . . . 100

3.2 Elution profiles for synthetic rock mixtures . . . . . . . . . . . 104

3.3 Pt stable isotopic measurements of replicate digestions of WPR-1, PTA- 1, SARM-6 and OKUM . . . . . . . . . . 107

$3.4 \quad$ Pt stable isotope variations in standard reference materials relative to IRMM-010 . . . . . . . . . . . . . . . . . . . . . . . 111

$4.1 \quad$ Pt stable isotope results for terrestrial and meteorite samples. 128

4.2 Modelling the effect of the late-veneer (LV) or addition of core material (CM) on the abundance and isotopic composition of Pt in the mantle. . . . . . . . . . . . . . . . 135

4.3 Model of Pt stable isotope evolution of Earth's core and mantle. 138 


\section{List of Tables}

1.1 Abundances of PGE in different environments. . . . . . . . . . 6

1.2 Key properties of chondrite groups . . . . . . . . . . . . . . 29

1.3 Thermal neutron capture cross sections of nuclides in the mass range of Pt. . . . . . . . . . . . . . 54

$2.1 \quad$ Isotopic composition (in \%) of natural platinum, Oak Ridge isotopic spikes and prepared double-spikes . . . . . . . . . 62

2.2 Instrumental operating and measurement conditions . . . . . . 67

$2.3 \quad$ Natural Pt isotope ratios measured for IRMM-010 . . . . . . . 69

2.4 Table of elemental doping test results . . . . . . . . . . . . . . 82

2.5 MC-ICPMS collector configurations and relevant interferences 89

3.1 Details of the standard reference materials analysed in this study. . . . . . . . . . . . . . . . . . . . . . 93

$3.2 \quad$ Pt stable isotope compositions of terrestrial standard reference materials. . . . . . . . . . . . . 108

3.3 Synthetic rock mixture compositions used for testing anion exchange chemistry. . . . . . . . . . . . . . . . 115

$3.4 \quad$ Pt stable isotope results for individual analyses, with weighted means for each replicate as given in the main text. . . . . . . 116

$4.1 \quad$ Pt stable isotope and isotope dilution results for terrestrial and extraterrestrial samples . . . . . . . . . . . . . . 127

4.2 Model parameters used in mixing models shown in Fig. 4.2]. . 134 


\section{Chapter 1}

\section{Introduction}

\subsection{Objectives of this thesis}

A number of new non-traditional stable isotope systems have been developed in recent years, largely as a result of the advent of multiple-collector inductively coupled plasma mass spectrometry (MC-ICPMS). Many of these new stable isotope tracers are providing valuable insights in the earth, environmental and planetary sciences (e.g. Halliday et al., 1998, Belshaw et al. 1998, 2000; Maréchal et al., 1999; Beard and Johnson, 1999; Zhu et al., 2000, Young and Galy, 2004; Williams et al., 2004; Anbar and Rouxel, 2007; Fitoussi et al., 2009). The platinum (Pt) stable isotope system represents a potentially powerful addition to this suite of stable isotopic tracers, with a variety of possible applications, yet it remains unexplored. The overlying aim of this thesis is to develop the necessary methods for measuring Pt stable isotope variations in geological materials and to make the first application of this new tracer to a geological problem. The geochemical behaviour of platinum makes it uniquely suited to investigating a range of different processes. In particular, Pt is strongly partitioned into planetary cores during planetary differentiation, and stable isotope ratios may provide key insights into the accretion and differentiation of Earth.

To enable Pt stable isotope measurements to be made, two major ana- 
lytical challenges first had to be overcome. First, reproducible methods for sample digestion and separation of $\mathrm{Pt}$ from various natural samples had to be established with sufficiently high yields and high purity of Pt. Second, analytical methods had to be developed for accurate determination of Pt stable isotope ratios, with sufficient precision to resolve small natural fractionations. The development of these techniques comprised a significant portion of this research, which is reflected in the structure of this thesis.

Once suitable techniques were established, Pt stable isotope compositions were measured in a range of terrestrial igneous and mantle samples and in different types of meteorites. The goals of this phase of research were: 1) to constrain the Pt isotopic compositions of the major terrestrial reservoirs, and the magnitude of Pt stable isotopic fractionations in high-temperature Earth processes; 2) to investigate the range of $\mathrm{Pt}$ stable isotopic variations amongst primitive meteorites (chondrites); 3) to evaluate the magnitude of isotopic fractionations associated with core formation in differentiated planetary bodies (achondrites and iron meteorites); and 4) to use the above data to test models of Earth's formation and differentiation and to place new constraints on these processes.

This introduction chapter provides information on the chemical and geochemical behaviour of platinum, which defines the areas in which these techniques may be applied; the formation of terrestrial planets, to provide a context for the problem to which the Pt stable isotope system is applied in this thesis; the meteorite samples that are used in this study; and case studies of several recently developed non-traditional stable isotope systems demonstrating how these can be applied to investigating planetary processes, including a discussion of the Pt stable isotope system in this context. 


\subsection{Geochemistry of platinum and the plat- inum group elements}

The platinum group elements (PGE; Rh, Ru, Pd, Os, Ir and Pt) are a cluster of transition metals in the periodic table (Fig. 1.1) that show coherent group behaviour that is distinct from their congeners ( $\mathrm{Fe}, \mathrm{Ni}, \mathrm{Co})$. They are all highly unreactive (noble) metals, being strongly resistant to forming oxygenated compounds. As a rule they are found associated together in nature, and generally uncombined (Clarke, 1920). In cosmochemical terms, they are all refractory with $50 \%$ condensation temperatures ranging from $1360 \mathrm{~K}$ (Pd) to $1800 \mathrm{~K}$ (Os) (Taylor, 2001); Pt is moderately refractory, with a 50\% condensation temperature of $1410 \mathrm{~K}$ (Taylor, 2001).

The PGE are highly siderophile, preferring bonds with iron over silicon and oxygen, and are preferentially removed into the metallic cores of planets during planetary differentiation. The relative distribution of highly siderophile elements (HSE; PGE plus Re and Au; Fig. 1.1) between the core and silicate mantle provides key constraints on models of planetary differentiation (Section 1.3). The PGE also exhibit chalcophile behaviour and in the conditions found in the mantle and crust they are most commonly found in sulphide components of ultramafic complexes. The geochemical behaviour of the PGE generally, and Pt specifically, is outlined briefly in this section with respect to different geological settings. This behaviour generally defines the types of geological problems to which the Pt stable isotope system may be applied.

\subsubsection{The mantle}

The mantle is an important geochemical reservoir, comprising $65 \%$ of the Earth's mass (Bennett, 2003). The composition of the mantle is characterised through studies of mantle derived peridotites sampled from orogenic massifs, abyssal oceanic peridotites and as xenolithic fragments in kimberlitic and 


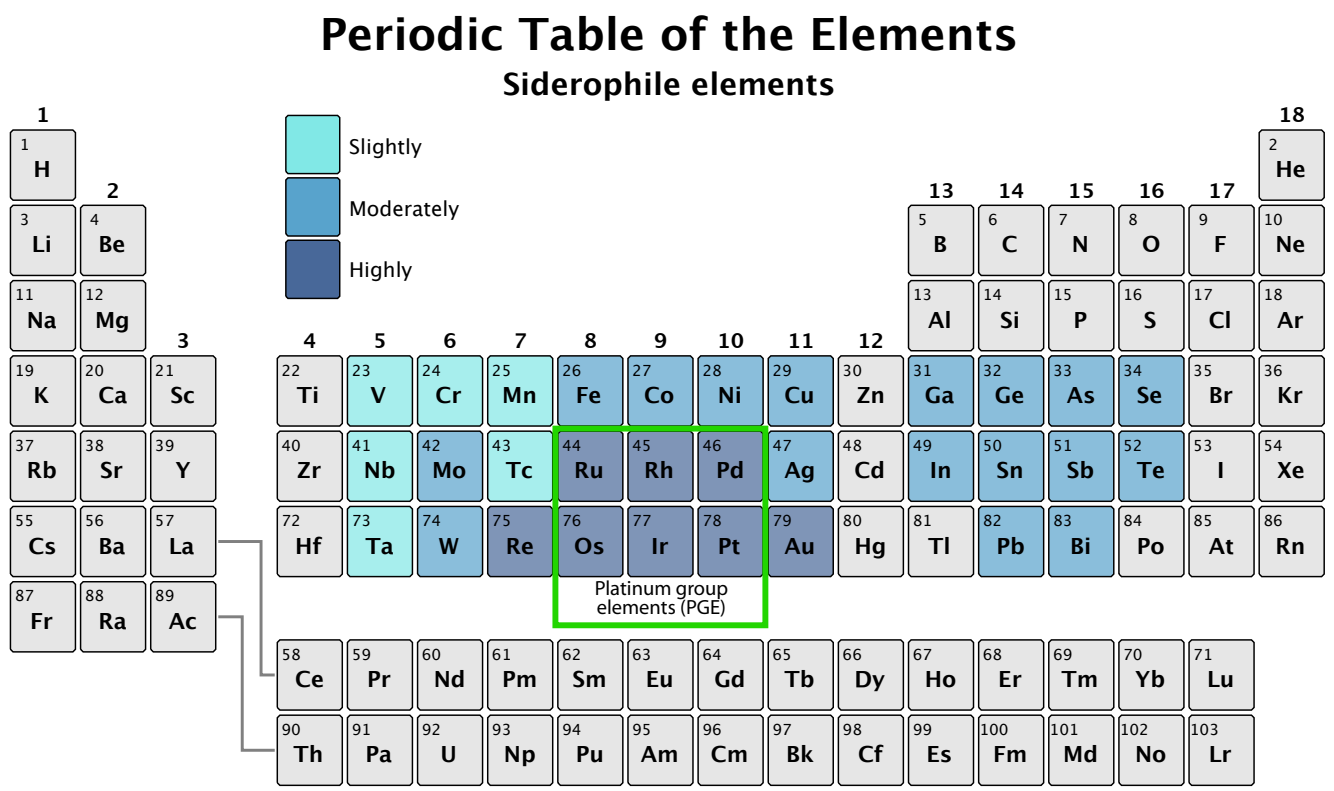

Figure 1.1: Periodic table of the elements showing slightly, moderately and highly siderophile elements. The highly siderophile elements (HSE) include the platinum group elements (PGE; indicated), Re and Au. Figure based on (Righter 2003).

basaltic rocks. These samples have been used to infer the composition of the 'primitive upper mantle' (PUM; Table 1.1), a hypothetical reservoir that has never undergone depletion by partial melting to form basalts (e.g., Becker et al. 2006 ${ }^{1}$. The abundances of HSE in the mantle have played a key role in constraining the accretion of Earth and the formation of the core. This aspect of the mantle geochemistry of HSE is discussed extensively in Section 1.3 , and therefore omitted from this section.

PGE analytical work in peridotites has been challenging due to their low concentrations (Table 1.1) and the presence of PGE-rich micro-nuggets (e.g., Mitchell and Keays, 1981; Lorand et al., 2008). Less than 10\% of PGE in the mantle are thought to be hosted in silicates (Handler and Bennett,

\footnotetext{
${ }^{1}$ Note: the term PUM is used in preference to bulk silicate earth (BSE) for the reasons outlined by Becker et al. (2006). This term is used in place of BSE throughout this introduction, including when published work is cited. The composition of PUM is discussed further in Section 1.3
} 
1999, Burton et al., 2002, Lorand et al., 2010). Within the range of oxygen and sulphur fugacities found in the upper mantle and crust, the PGE show strongly chalcophile behaviour and are strongly concentrated in (volumetrically minor) Fe-Ni-Cu metal sulphide phases in the mantle (Naldrett and Duke, 1980; Ballhaus and Stumpfl, 1986; Handler and Bennett, 1999; Alard et al., 2000; Lorand and Alard, 2001; Lorand et al., 2008; Mondal, 2011). Peridotite samples from the upper mantle show considerable variation in chemical and mineralogical composition. These variations largely represent physical processes in the mantle such as melt extraction, metasomatism and mixing between distinct mantle reservoirs (e.g., Shirey and Walker, 1998; Handler and Bennett, 1999; Luguet et al., 2001; Bennett, 2003; Becker et al. 2006: Mondal, 2011). Radiogenic isotope (e.g., ${ }^{187} \mathrm{Re}^{-}{ }^{187} \mathrm{Os},{ }^{190} \mathrm{Pt}-{ }^{186} \mathrm{Os}$ ) systematics of HSE have provided important tools for investigating these mantle heterogeneities and mantle dynamics (Shirey and Walker, 1998; Walker, 1997; Pearson et al., 2004; Lorand et al., 2008), potential interactions between the core and mantle (Walker et al., 1995, Brandon et al., 1998), and intraplate volcanism (e.g., Day, 2013). Mantle melting is the most important Earth process for fractionating the HSE (Lorand et al., 2008). Subtle differences in the geochemical behaviour of PGE during melting in the mantle leads to variations in their relative abundances in different mantle and mantle-derived samples, and these have also been important for tracing mantle processes (Mitchell and Keays, 1981; Rehkämper et al., 1997, 1999a b; Handler and Bennett, 1999; Lorand and Alard, 2001; Luguet et al., 2001; Lorand et al. 2008, 2010).

\subsubsection{Igneous systems}

Undepleted fertile peridotites have near-chondritic relative abundances of the PGE, and deviations from these abundances in mafic and ultramafic rocks reflect fractionations of PGE relative to one another during partial melting, magma differentiation and fractional crystallisation. These processes have been investigated by studying PGE compositions of peridotites and mantle- 
Table 1.1: Abundances of PGE in different environments.

\begin{tabular}{|c|c|c|c|c|c|c|}
\hline Element & $\mathrm{Ru}(\mathrm{ppb})$ & $\mathrm{Rh}(\mathrm{ppb})$ & $\mathrm{Pd}(\mathrm{ppb})$ & Os (ppb) & $\operatorname{Ir}(p p b)$ & $\mathrm{Pt}(\mathrm{ppb})$ \\
\hline CI chondrite ${ }^{a}$ & 710 & 130 & 550 & 490 & 455 & 1010 \\
\hline Iron meteorites ${ }^{\mathrm{b}}$ & 10600 & 2200 & 4000 & 15400 & 10400 & 14700 \\
\hline Bulk Earth ${ }^{c}$ & 1310 & 242 & 1015 & 900 & 835 & 1866 \\
\hline Core $^{c}$ & 4000 & 740 & 3100 & 2750 & 2550 & 5700 \\
\hline BSE / PUM ${ }^{c}$ & 4.97 & 0.91 & 3.85 & 3.43 & 3.19 & 7.07 \\
\hline Continental crust $^{\mathrm{d}}$ & 0.10 & 0.06 & 0.40 & 0.05 & 0.05 & 0.40 \\
\hline Manganese nodules ${ }^{e}$ & 8 & 13 & 6 & 2 & 7 & 200 \\
\hline Pelagic sediments ${ }^{\mathrm{e}}$ & 0.2 & 0.4 & 6.0 & 0.1 & 0.4 & 5.0 \\
\hline Seawater ${ }^{\mathrm{f}}(\mathrm{ng} / \mathrm{L})$ & & & 0.0426 & 0.0017 & 0.00115 & 0.117 \\
\hline
\end{tabular}

Data sources: ${ }^{2}$ McDonough and Sun (1995); ${ }^{b}$ Based on average values from D'Orazio and Folco (2003) and Petaev and Jacobsen (2004); ‘ McDonough (2003); d Wedepohl (1995); ${ }^{\mathrm{Li}}(1991) ; \sqrt{\text { Albarède }}$ (2003).

derived lavas such as komatiites and basalts. The role of different major or minor mineral phases in these fractionations is widely discussed in the literature (e.g., Barnes and Picard, 1993, Brenan et al., 2003, Handler and Bennett, 1999, Pearson et al., 2004; Puchtel and Humayun, 2000, 2001; Rehkämper et al., 1999b).

The PGE are often separated into two groups that show different group behaviour: iridium-PGE (IPGE: Ir, Os, Ru) and palladium-PGE (PPGE: Pt, $\mathrm{Rh}, \mathrm{Pd})$. Re shows similar geochemical behaviour to the PPGE, and is often included in studies of PGE in igneous systems. PPGE and IPGE are generally considered to be controlled by separate phases in mafic and ultramafic igneous systems. The IPGE are more refractory and are generally compatible in silicate (olivine) during partial melting (Barnes and Picard, 1993; Rehkämper et al., 1999b; Puchtel and Humayun, 2001; Brenan et al., 2003), but may also be retained in refractory Os-Ir alloy phases (Barnes et al., 1985, Barnes and Picard, 1993; Rehkämper et al., 1999b) or Ru-Os-Ir sulphides (Pearson et al. 2004). The PPGE and Re are incompatible during melt extraction, and are thought to be dominantly controlled by partitioning into sulphide phases (e.g., Jagoutz et al., 1979: Pattou et al., 1996; Handler and Bennett, 1999, Puchtel and Humayun, 2001; Pearson et al., 2004). Sulphur-saturation in a magma leads to the development of an immiscible sulphide liquid which 
concentrates the PPGE (Rehkämper et al., 1999b). Komatiite magmas are considered to be S-undersaturated, while basalts are generally considered to be S-saturated, resulting in higher and lower PPGE concentrations, respectively (Rehkämper et al., 1999a; Puchtel and Humayun, 2001).

The different behaviour of PPGE and IPGE during melt extraction results in highly depleted PPGE in the residual peridotite and highly fractionated chondrite-normalised PGE patterns (e.g., Rehkämper et al., 1999b; Pearson et al. 2004). Most mafic extrusive rocks have $\mathrm{Pd} / \mathrm{Pt}$ ratios greater than the mantle, indicating that $\mathrm{Pt}$ is generally more compatible during partial melting than Pd (Barnes and Picard, 1993). In general, Ir is considered the most compatible PGE and Pd is the least compatible, with compatibility decreasing in the order $\mathrm{Ir} \sim \mathrm{Os} \sim \mathrm{Ru}>\mathrm{Pt}>\mathrm{Pd}$ (Rehkämper et al., 1999b; Pearson et al. 2004). Other minor phases, such as spinel and chromite, may play a minor role in fractionation of PGE (e.g., Puchtel and Humayun, 2001).

\subsubsection{Platinum ores}

PGE are relatively enriched in ultramafic rocks compared with intermediate and mafic rocks (Table 1.1), and most economic concentrations of PGE are associated with large ultramafic complexes (Maier, 2005; Mondal, 2011). As described above, the PGE show a strong affinity for sulphides under mantle conditions, and commonly exist as solid-solutions with base metal sulphides and as sulphide minerals (Fleet et al. 1999a b; Mondal, 2011; Brenan and McDonough, 2005; Maier, 2005; Godel et al., 2007). PGEs can form >150 mineral species, including native metals, alloys, arsenides, tellurides, selenides, antimonides and oxides, as well as sulphides (Bannister, 1932 ; Cabri, 1992 Mondal, 2011). Important Pt mineral phases in ore deposits include sulphides, such as cooperite $((\mathrm{Pt}, \mathrm{Pd}) \mathrm{S})$, braggite $((\mathrm{Pt}, \mathrm{Pd}, \mathrm{Ni}) \mathrm{S})$ and malanite $\left(\mathrm{Cu}(\mathrm{Pt}, \mathrm{Ir}, \mathrm{Co})_{2} \mathrm{~S}_{4}\right)$, arsenides, such as sperrylite $\left(\mathrm{PtAs}_{2}\right)$, and tellurides, such as moncheite $\left((\mathrm{Pt}, \mathrm{Pd})(\mathrm{Te}, \mathrm{Bi})_{2}\right)$ (Bannister, 1932, Anthony et al., 2004, Mondal, 2011). Pt is not readily incorporated into silicate structures because of its inability to form stable ionic bonds (Hagen, 1954). Magmatic PGE ores 
are thought to form during generation of ultramafic magmas by gravity separation of denser sulphide phases which form an immiscible sulphide liquid. The PGE are concentrated in the immiscible sulphide liquid in which major sulphide minerals (e.g., pyrrhotite, chalcopyrite, pentlandite and pyrite) form accompanied or followed by the crystallisation of discrete PGE minerals (Naldrett and Duke, 1980). Economic deposits of PGE are also found in secondary 'placer' deposits in alluvium (Mondal, 2011).

\subsubsection{Marine environment}

$\mathrm{Pt}$ and $\mathrm{Pd}$ in the oceans are thought to exist primarily in the divalent $(+2)$ state as anionic chlorocomplexes, as a consequence of the high concentration of chloride ion in seawater (Goldberg, 1987). Pt is relatively unreactive in the water column, forming stable chloro- and bromo-complexes, and hence has a long residence time in the ocean $\left(10^{6} \mathrm{y}\right.$; Goldberg et al., 1986; Colodner, 1991). Enrichments in Pt relative to the other PGE have been observed in authigenic marine sediments such as ferromanganese crusts and nodules (Table 1.1). In modern oxic environments, these ferromanganese concretions form as $\mathrm{Mn}$ is oxidised from the +2 to the +4 state by dissolved oxygen gas (Goldberg, 1987). These ferromanganese concretions incorporate other oxidisable metals, in particular $\mathrm{Ca}, \mathrm{Pb}$ and $\mathrm{Co}$, but also the PGE (particularly $\mathrm{Pt}$, and to a lesser extent $\mathrm{Ru}$ and Ir). Platinum, which can be oxidised to the tetravalent $(+4)$ state, is markedly enriched (an order of magnitude or more) in ferromanganese nodules over $\mathrm{Pd}$, which is thought to only occupy the divalent state in the ocean (Table 1.1; Hodge et al., 1985, Goldberg, 1987; Colodner, 1991; Banakar et al., 2007). Fe-Mn crusts are the major sink for Pt in the modern oxic oceans.

\subsubsection{Hydrothermal systems}

High concentrations (ca. 3-4 ppm) of Pt have been found in hydrothermal copper and nickel deposits associated with mafic igneous rocks McCallum 
et al., 1976, Rowell and Edgar, 1986). A geochemical study of such deposits showed that $\mathrm{Pt}$ and $\mathrm{Rh}$ are substantially enriched at strongly oxidised ore horizons (McCallum et al., 1976). The solubility of $\mathrm{Pt}$ is dependent on the temperature and chemistry of the hydrothermal fluid as well as redox conditions. Pt can be present as chloride complexes, particularly $\mathrm{PtCl}_{4}{ }^{-2}$ and $\mathrm{PtCl}_{6}{ }^{-2}$ (complexes of $\mathrm{Pt}(\mathrm{II})$ and $\mathrm{Pt}(\mathrm{IV})$, respectively; Gammons, 1996), or bisulphide and/or hydroxide complexes (Mountain and Wood, 1988). Gammons (1996) suggested that the stability of Pt(II) and Pt(IV) chloride complexes does not change with temperature, and that the stability of $\mathrm{Pt}(\mathrm{IV})$ in hydrothermal fluids is dependent on the aqueous Pt concentration. Thus, Pt(IV) may be rare in high-temperature hydrothermal fluids (Mountain and Wood, 1988; Gammons, 1996). However, under certain (strongly oxidised) conditions, Pt(IV) may be important in ore-forming environments Jaireth. 1992; Gammons, 1996).

\subsubsection{Meteorites}

PGE concentrations in iron meteorites are high compared with chondrites, reflecting the siderophile nature of these elements during formation of planetary cores (Table 1.1). The siderophile nature of PGE is also expressed in their relatively low concentrations in the mantle compared with meteorites (Table 1.1). The relationship between meteoritic PGE abundances and the composition of Earth's mantle is discussed further in Sections 1.3 and 1.4). The relatively high concentrations of PGE in extraterrestrial materials has led to their use as indicators of cosmic inputs, particularly meteorite impacts, in sedimentary horizons (e.g. Evans and Chai, 1997, and references therein). One of the best known examples of this is the enrichment in Ir found in deposits from the Cretaceous-Paleogene boundary, which led to the meteorite impact hypothesis for the mass extinction event at that time Alvarez et al. 1980). Pt-Re-Os systematics in iron meteorites have also been used to investigate fractional crystallisation in Fe-Ni metallic cores of asteroid parent bodies (Cook et al., 2004). 


\subsection{Terrestrial planet formation and evolu- tion}

While the processes of planetary accretion and differentiation are broadly understood, questions remain regarding the nature of the building blocks that accreted to form Earth and the relative timing and physical processes of planetary differentiation. Key constraints on models of Earth's accretion and differentiation come from the distribution of siderophile elements between the core and the mantle. Various, conflicting, models exist that seek to explain the observed siderophile element distributions. One objective of this thesis is to apply the $\mathrm{Pt}$ stable isotope system to look for isotopic variations in terrestrial and extraterrestrial samples in order to test such models. This section describes the broad processes of terrestrial planet formation and then details the existing models for the accretion and differentiation of Earth with the relevant supporting evidence.

\subsubsection{Solar System and planet formation}

The nebular hypothesis is the most widely accepted model for the formation and evolution of the solar system. In this model, the Sun formed by the gravitational collapse of a relatively small region of a giant molecular cloud, perhaps occurring spontaneously or potentially triggered by a nearby supernova explosion (Cameron and Truran, 1977). Cosmochemical, astrophysical and astronomical studies have placed constraints on the astrophysical environment, timescales and the processes of solar system formation. As the molecular cloud condensed around the forming Sun, it formed a rotationally supported disk in which the planets formed by accretion of disk material. Such protoplanetary disks have now been observed in a number of star forming regions in our galaxy (Fig 1.2). Terrestrial planets formed within the inner, hot part of the disk, which was enriched in refractory materials (silicates and metals), while gas planets formed in the outer part of the disk where 

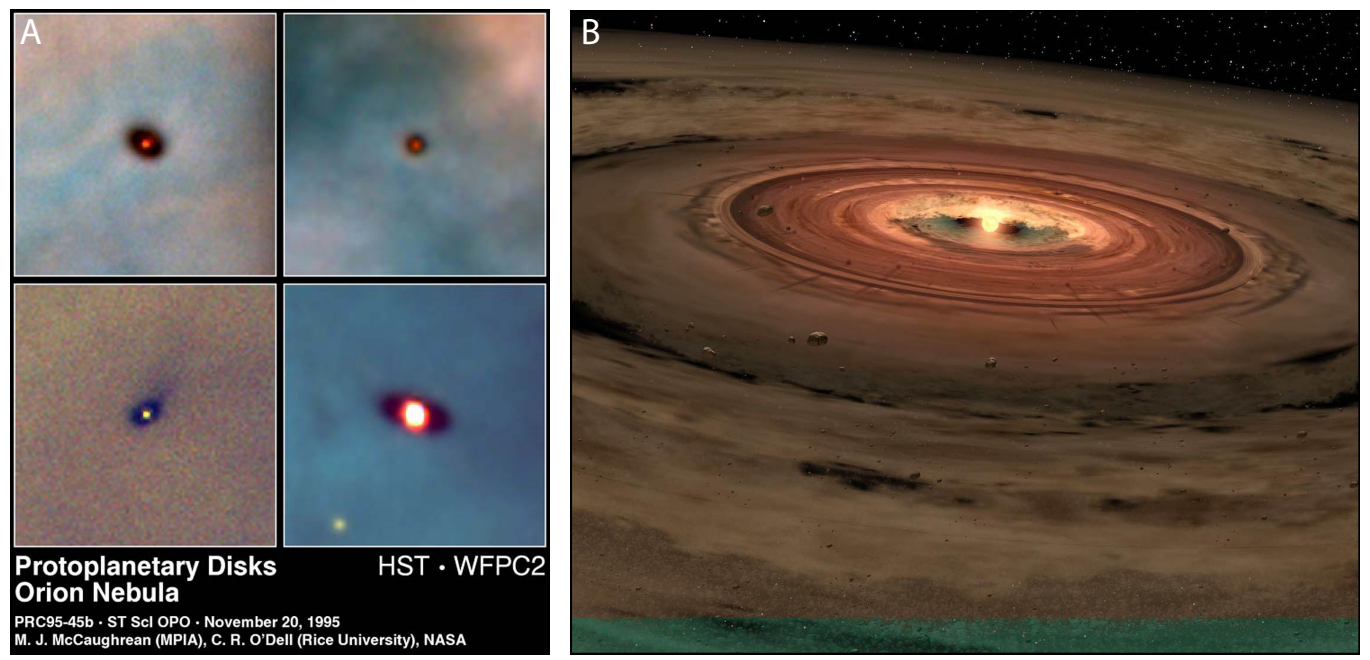

Figure 1.2: A) Examples protoplanetary disks in active star forming regions of our galaxy as imaged by the Hubble Space Telescope. Image credit: NASA. B) Artists impression of a protoplanetary disk. Accretion of terrestrial planets occurs in the region near to the star, where only metals and silicates can condense. Image credit: NASA.

cooler temperatures allowed gases and water ice to condense. The first planetesimals (up to ca. $1 \mathrm{~km}$ in diameter) formed within ca. 1-2 Myr (Bizzarro et al., 2005, Scherstén et al., 2006). Once planetesimals formed, they interacted with one another through gravity, and the largest bodies grew at the highest rate, known as runaway growth (Kokubo and Ida, 2002). When the largest bodies exceeded $1000 \mathrm{~km}$, runaway growth slowed down and the growth mode changed to oligarchic growth, where just the largest bodies continue to accrete planetesimals (Kokubo and Ida, 2002). These large planetary embryos gradually collided to form the terrestrial planets (Chambers, 2007).

\subsubsection{Accretion and differentiation of terrestrial plan- ets}

It is generally accepted that Earth accreted from nebular condensates that are best represented by primitive undifferentiated meteorites, i.e., chondrites (e.g., Drake and Righter, 2002, Halliday, 2003; for details of meteorite clas- 
sification see Section 1.4. CI chondrites have elemental compositions that closely match the solar photosphere (Anders and Grevesse, 1989, Grevesse and Sauval, 1998), and are often taken to represent the composition of the solar nebular and, thus, the undifferentiated composition of Earth. However, no group of primitive meteorites provides a perfect match for Earth. Large variations are observed in oxygen isotope compositions of the different groups of chondrites (see Section 1.4.2), and Earth and the Moon have distinct compositions from carbonaceous chondrites (Clayton, 2003). Enstatite chondrites are the only group that lie on the same O isotopic fractionation line as the Earth and Moon (Clayton et al. 1984), and while they have distinctly different elemental chemical compositions from Earth, enstatite chondrites are found to have very similar isotopic compositions for a number of other elements (N, Mo, Ru, Os and Ti) (Javoy et al., 2010, and references therein). Ordinary chondrites also have isotopic similarities to Earth, in $\mathrm{Ti}$ (Leya et al., 2008) and Sm (Carlson et al., 2007) isotopes for example. It is likely that Earth accreted a variety of different primitive materials (Albarède, 2003). However, chondrites provide a useful comparison for approximating bulk Earth composition, and most studies use the mean CI chondrite composition to represent the composition of Earth (Drake and Righter, 2002 Albarède, 2003; Halliday, 2003).

As discussed in Section 1.2.1, the term primitive upper mantle (PUM) represents a hypothetical composition that defines the composition of the silicate Earth after core formation and before any extraction of melts to form the crust. This term is preferred by some workers (as opposed to primitive mantle or bulk silicate Earth) as it does not assume that the lower mantle has the same composition (e.g., Greenough and Owen, 1992, Becker et al., 2006 Walker, 2009). The composition of PUM is estimated by extrapolation from measured compositions of mantle peridotite samples, which are derived from the upper mantle (e.g., Fig 1.3, Becker et al., 2006; Walker, 2009). The goal of accretion and differentiation models is to find mechanisms that can reproduce the PUM from a chondritic starting composition. 


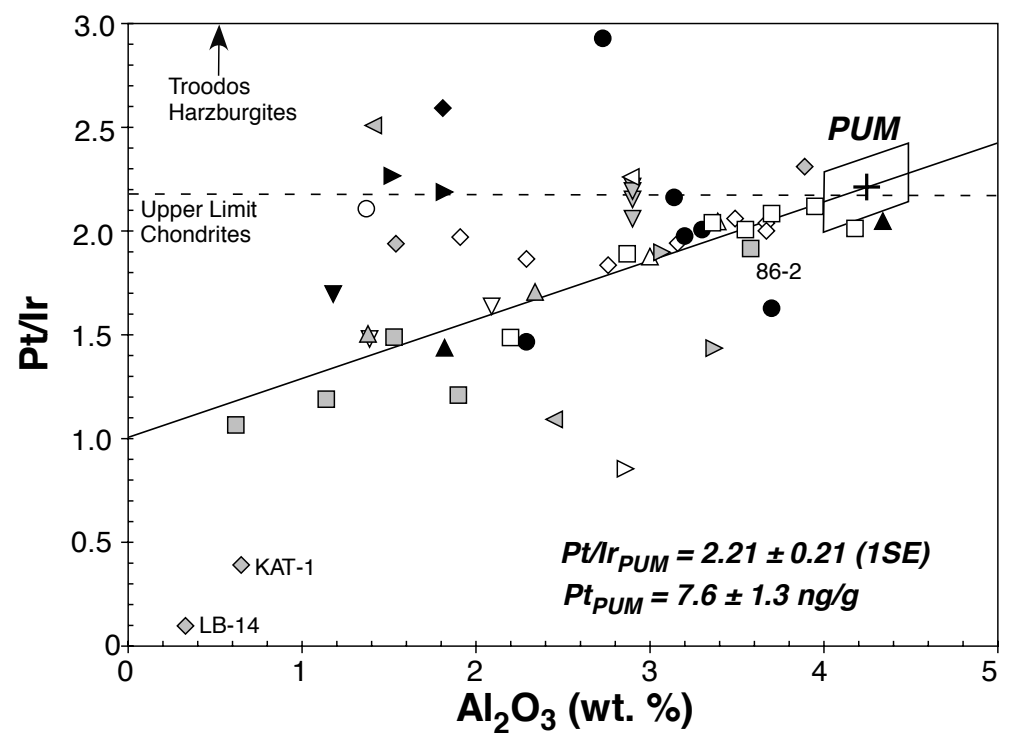

Figure 1.3: $\mathrm{Pt} / \mathrm{Ir}-\mathrm{Al}_{2} \mathrm{O}_{3}$ diagram from Becker et al. (2006), showing how the composition of PUM is estimated by extrapolation of measured peridotite data using an index for the degree of melt extraction, in this case $\mathrm{Al}_{2} \mathrm{O}_{3}$.

Timescales of planet formation and differentiation are provided by radioactive decay systems. The absolute age information of the formation of planets are provided by a range of long-lived chronometers (Wadhwa, 2007). The ${ }^{235,238} \mathrm{U} \_207,206 \mathrm{~Pb}$ system is notable, as it provides the highest time resolution of all the long-lived chronometers that have been applied to dating early solar system materials (e.g., Patterson, 1956; Amelin et al., 2002; Connelly et al., 2008, 2012). A number of short-lived (now extinct) radionuclides were present in the early solar system (McKeegan and Davis, 2003), and materials that formed while these radionuclides were live contain measurable excesses of their daughter nuclides, providing high temporal resolution chronometers for solar system processes, including planetary differentiation. For example, the decay of ${ }^{107} \mathrm{Pd}$ to ${ }^{107} \mathrm{Ag}$ has a half-life of $6.5 \mathrm{Myr}$, and the presence of ${ }^{107} \mathrm{Ag}$ excesses in iron meteorites has been used to show that planetesimals accreted, melted and differentiated while ${ }^{107} \mathrm{Pd}$ was still live in the Solar System, i.e., within ca. 10 Myr (Chen and Wasserburg, 1990). The 
hafnium-tungsten system is based on the decay of short-lived ${ }^{182} \mathrm{Hf}$ to ${ }^{182} \mathrm{~W}$ with a half-life of ca. 9 Myr (McKeegan and Davis, 2003; Kleine et al., 2004b). The Hf-W system is ideal for tracing core formation in planets as the parent, Hf, is lithophile while the daughter, $\mathrm{W}$, is siderophile, and thus the parent and daughter are fractionated by removal of metals into the core. Hf-W studies suggest core formation on Mars took just 5-20 Myr (Halliday et al., 2001; Kleine et al., 2004a; Dauphas and Pourmand, 2011), and core formation on Earth was largely complete by ca. 30 Myr after the start of the Solar System (Kleine et al. 2002; Yin et al., 2002, Kleine et al., 2004b). Most models consider that core formation was complete by the time of the Moon-forming impact (Hartmann and Davis, 1975, Cameron and Ward, 1976, Stevenson. 1987; Benz et al., 1987; Newsom and Sims, 1991; Canup and Asphaug, 2001; Halliday et al. 2000), and Hf-W and Sm-Nd isotopic evidence suggest that the Moon formed around 60-110 Myr after the origin of the Solar System (Touboul et al., 2007; Halliday, 2008; Halliday and Wood, 2009).

A number of fundamental questions over the details of Earth's accretion and differentiation remain outstanding. For example: What was the nature of the building blocks that accreted to form Earth? Did the nature of the accreting material change significantly in composition over time? What were the physical conditions and mechanisms of core formation? Did the core form in equilibrium with the mantle? Did core formation occur in a single event or continuously as Earth accreted? Was the accreting material unmodified primitive material or did the Earth accrete smaller differentiated bodies with pre-existing cores? An ideal model should be able to answer all of these questions.

\subsubsection{Models for Earth's accretion and differentiation}

Key constraints on accretion and core formation come from siderophile elements. Siderophile element abundances in chondrites are several orders of magnitude higher than those of the PUM (Fig. 1.4). The relative depletion of the siderophile elements in PUM compared with chondrites, as well 
as the relatively high siderophile element concentrations in iron meteorites, suggests that most of Earth's siderophile elements were partitioned into the core during core formation. Similar depletions are observed in the silicate components of all differentiated bodies for which we have samples (Righter and Drake, 1996). Experimental determinations of the partitioning behaviour of siderophile elements between metal and silicate at low pressure and moderate temperature (e.g., Capobianco et al., 1993) suggest that metal-silicate differentiation should have quantitatively removed these elements from the mantle (Borisov et al. 1994; Ertel et al., 2006, Righter et al., 2008; Brenan and McDonough, 2009; Holzheid et al., 2000; Cottrell and Walker, 2006; Ohtani et al., 1997; Fortenfant et al., 2003). It has long been recognised that siderophile element abundances in the PUM are significantly higher than would be expected if the core formed under low pressure and low temperature equilibrium conditions (Chou, 1978; Jagoutz et al., 1979). This has become known as the 'excess siderophile elements problem' (e.g. Capobianco et al., 1993). The discrepancies between predicted and observed abundances are a factor of 10-100 for moderately siderophile elements (e.g., Ni, Co, W, Mo) and a factor of $>1000$ for highly siderophile elements ( $\mathrm{Ru}, \mathrm{Rh}, \mathrm{Pd}, \mathrm{Re}$, Os, Ir, Pt and Au; Fig. 1.4). Mechanisms to explain these abundances are a key component of terrestrial accretion models.

The composition of Earth's core is inferred from mass balance with the mantle and by geophysical observations. Geophysical measurements show that Earth's core is less dense than would be expected for pure Fe-Ni metal, and thus the core must contain ca. $5-10 \%$ of a light element to explain the mass deficit. Proposed candidates for the light element present in the core include H, C, O, S and Si (e.g., Allègre et al., 1995; McDonough, 2003) or some combination of these. The abundant light element in the core is of importance for accretion models as it determines whether core formation was dominated by high pressure or low pressure processes. For example, presence of $\mathrm{S}$ in the core indicates low pressures and thus core formation may have been occurring during accretion (Newsom and Sims, 1991). By contrast, Si 


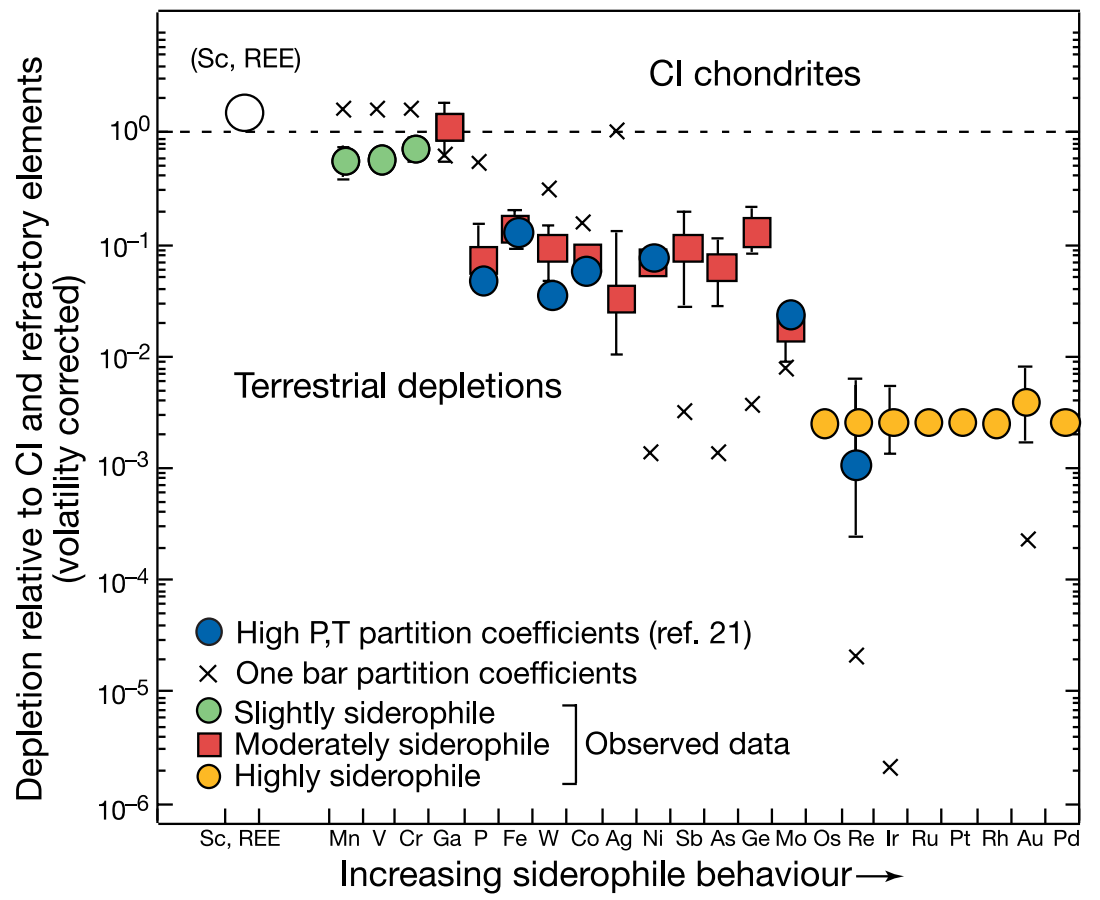

Figure 1.4: A 'depletion' diagram showing the abundances of elements in Earth's upper mantle compared with chondrites, corrected for incomplete incorporation into Earth because of relative volatility. Figure modified from Drake and Righter (2002).

may only enter the core under extremely high pressure conditions (Allègre et al., 1995; McDonough, 2003, Gessmann et al., 2001; Fitoussi et al., 2009, Armytage et al., 2012).

The following models all have geochemical consequences that must be taken in to account, and no existing model is consistent with all observations. Most workers agree that some combination of these processes likely occurred.

\section{Core formation}

Early models for the accretion of terrestrial planets were divided between two broad mechanisms: homogeneous accretion and heterogeneous accretion. Homogeneous accretion models assume that the terrestrial planets accreted from nebular condensates similar to primitive meteorites, and the accreting material did not significantly change in composition over time (i.e., accret- 
ing material sampled the same mixture of compositions over time). Core formation occurred either during or after accretion by sinking and coalescence of molten metal. To allow metals to sink into the core, temperatures must have been high enough to cause partial melting. Heat sources during the accretion process were likely abundant. Accretional energy would have provided a significant amount of heat, with the forming planet accreting objects up to one third of its mass (Wetherill, 1985; Tonks and Melosh, 1992). The sinking of dense material towards the core would also have released a significant amount gravitational potential energy as heat (Ringwood, 1966. Brett, 1976: Tonks and Melosh, 1992). Metals are thought to have collected in a viscous boundary layer, with large masses of metal sinking to the core as metal diapirs. Some models include a magma ocean of varying scales up to global scale (e.g., Newsom and Sims, 1991). In homogeneous accretion models, the metallic core should be in equilibrium with the silicate mantle, and thus siderophile element depletions are expected, although the magnitude of these depletions depends on the partitioning behaviour of those elements under the conditions of core formation, which is discussed further below.

A proposed alternative to homogenous accretion models is heterogeneous accretion. Early heterogeneous accretion models predicted, based on nebular condensation theory, that metal should condense first out of a hot nebular gas, followed by silicates (Eucken, 1944). Eucken's and other subsequent models suggested that the core material was the first to condense from the solar nebula, and then silicates subsequently condensed and accreted to the planet forming the mantle (e.g., Eucken, 1944, Turekian and Clark, 1969). Heterogeneous accretion models have continued to be developed, and the most widely supported version of the this model is attributed to Wänke and others (e.g., Wänke and Gold, 1981; Wänke et al., 1984). In their model, the first $80-90 \%$ of Earth accreted from very reduced materials, where most elements were present as metals and all but the very lithophile elements would be extracted into the core. The next 10-20\% was progressively more oxidised, and all but the most siderophile elements would be retained in the 
mantle. The final ca. $1 \%$ of accreting material would be so oxidised that virtually no metallic Fe would be present, and even the highly siderophile elements would be retained in the mantle (Wänke and Gold, 1981, Wänke et al., 1984). This model can thus explain the stair-step distribution of the siderophile elements in the terrestrial upper mantle (Fig. 1.4). It may also be dynamically plausible that accretion was fed from different spatial (compositional) regions over time (Drake, 2000, Drake and Righter, 2002 Chambers, 2007).

These models suggest different degrees of equilibration between Earth's core and mantle. Heterogeneous accretion models allow core formation with little or no core-mantle equilibration. Models of metal-silicate differentiation during or at the end of homogeneous accretion suggests that the core should have formed in chemical equilibrium with the mantle. The planetesimals accreting to form Earth may have already undergone metal-silicate differentiation to varying degrees, and disequilibrium models of core formation suggest that the metallic cores of these planetary embryos could directly enter Earth's core without equilibrating with Earth's mantle (Rudge et al. 2010). Compositional differences may also exist between the upper and lower mantle (Walker, 2009). The degree of equilibrium between these major reservoirs affects how observations should constrain accretion models.

\section{Late-veneer}

The relative abundances of siderophile elements in the mantle are similar to chondrites. For this similarity to result from metal-silicate fractionation is considered too coincidental by some authors (e.g., Jagoutz et al., 1979), who suggest that processes occurring during core formation would be expected to overprint the chondritic signature. An alternative explanation for both the absolute and relative siderophile element abundances is proposed by the the 'late-veneer' hypothesis. As in heterogeneous accretion models, the lateveneer hypothesis proposes that the last ca. $0.5 \%$ (ca. $10^{22} \mathrm{~kg}$ ) of Earth material was accreted after core formation (e.g. Kimura et al., 1974, Chou, 
1978). Mixing of this siderophile-rich late-veneer material into the mantle may explain the siderophile element abundances. The exact composition of the late-veneer is unknown although suggested compositions have included carbonaceous (Chou, 1978; Morgan, 1986, Morbidelli et al., 2000; Dauphas and Marty, 2002), enstatite (Shirey and Walker, 1998; Meisel et al., 1996; Walker et al., 2001, Righter, 2003; Fischer-Gödde et al., 2011) and ordinary (Meisel et al., 1996, Shirey and Walker, 1998; Walker et al., 2001; Drake and Righter, 2002, Fischer-Gödde et al., 2011) chondrite material, or combinations of these. Some have also suggested that cometary material may have played a role to some extent (Morbidelli et al., 2000; Dauphas and Marty, 2002 Drake and Righter, 2002). Estimates of the mass of late-veneer material required to reproduce siderophile element and noble gas abundances vary from ca. $0.1-1.0 \%$ of Earth's total mass (Chou, 1978; Holzheid et al., 2000; Dauphas and Marty, 2002). Mantle concentrations of siderophile elements, as measured in Proterozoic and Archaean komatiites, appear to have increased through Earth's early history up until ca. 2.9 Gyr ago, which has been interpreted to reflect progressive mixing of a late-veneer into the mantle (Maier et al. 2009). This has also been supported by recent $\mathrm{W}$ isotope evidence, which shows small $\mathrm{W}$ isotopic enrichments that can be reconciled with the present day mantle composition by addition of chondritic material (Willbold et al. 2011). Studies of siderophile element abundances in meteorite samples suggest that late accretion may have similarly affected other differentiated bodies in the Solar System (Walker et al., 2004, Day et al., 2012, Dale et al. 2012).

The late-veneer may be linked to a period of late accretion in the inner solar system known as the Late Heavy Bombardment (LHB) or Lunar cataclysm. The Moon, despite forming after the main accretionary period of the terrestrial planets, is heavily cratered. This indicates that there must have been a period of intense bombardment in the inner Solar System after the time the Moon formed. Dating of impact melt rocks brought back by the Apollo missions suggests that most of the Lunar cratering occurred within a 
narrow interval of time (Tera et al., 1974; Wetherill, 1975; Hartmann et al. 2000). The LHB may have been triggered by migration of the giant planets that destabilised a zone of planetesimal debris (Gomes et al., 2005), or may have occurred over a longer interval fed by a stock of planetesimals that inhabited highly-inclined orbits (Morbidelli et al. 2001). This hypothesised late influx of meteoritic material is presumed to have similarly been delivered to Earth and the other terrestrial planets (e.g., Kring and Cohen. 2002). The late-veneer has also been suggested to provide a low-temperature mechanism for delivering volatile elements and compounds (e.g. water) to Earth (Drake and Righter, 2002; Albarède, 2009; Schönbächler et al., 2010. Wang and Becker, 2013). However, the LHB is not universally accepted (e.g., Hartmann, 1975, 2003, Norman, 2009), and alternative hypotheses for the delivery of water and volatiles to the inner Solar System have been proposed (Morbidelli et al., 2000; Schönbächler et al., 2010).

While a late-bombardment almost certainly did occur (as evidenced by cratering on the Moon), there is a question over whether it had sufficient mass to to account for the siderophile element abundances in Earth's mantle Kleine, 2011). In addition, the late-veneer model has also been challenged on the viability of physically mixing the late-veneer into the upper mantle (Jones and Drake, 1986), although this problem may be overcome if the late bombardment resulted in a magma ocean (Walker et al., 1993).

\section{Metal-silicate equilibration in a magma ocean}

The major problem with early models of equilibrium core formation was their inability to explain the siderophile element abundances in the mantle, which could not be reconciled with low pressure and moderate temperature partition coefficients. However, it has long been recognised that the partitioning behaviour of the siderophile elements may be different under higher pressure and temperature conditions or different oxygen fugacities in the lower mantle (e.g., Brett, 1971; Ringwood, 1977; Brett, 1984; Murthy, 1991; Capobianco et al. 1993; Righter and Drake, 1997; Li and Agee, 2001). These factors 
could result in less removal of siderophile elements from the mantle during core formation, potentially obviating the need for a chondritic late-veneer. Early considerations of high pressure and temperature partitioning relied on extrapolations from low pressure and moderate temperature experimental data (e.g., Murthy, 1991, Capobianco et al., 1993). Over recent years the experimental database of element partitioning data has been extended to higher pressures and temperatures and a range of oxygen fugacities, which have yielded reduced partition coefficients for highly siderophile elements (e.g., $\mathrm{D}(\mathrm{Pt})_{\text {met/sil }} \approx 10^{3}-10^{4}$ compared with ca. $10^{8}-10^{13}$ for low temperature/pressure partitioning; Righter, 2003; Cottrell and Walker, 2006; Walker, 2009). These have led to new models of core formation with metal-silicate equilibration in a deep (possibly hydrous) magma ocean (Figure 1.5;e.g., Schmitt et al., 1989; Murthy, 1991; Ohtani et al., 1997; Righter and Drake, 1999 Cottrell and Walker, 2006; Righter et al., 2008; Wood et al., 2008; Halliday and Wood, 2009, Cottrell et al., 2009; Righter, 2011; Righter et al. 2011; Rubie et al., 2011). These studies suggest that siderophile element abundances can be reconciled in scenarios with an inferred magma ocean of intermediate depth, e.g., 750-1100km (25-30 GPa, 1900-2100 ${ }^{\circ} \mathrm{K}$; Li and Agee, 1996, Righter and Drake, 1997; Ohtani et al., 1997), or higher pressures and/or temperatures in a magma ocean of ca. 1200-1450 km depth (40-60 GPa and 2200-4000 ${ }^{\circ} \mathrm{C}$; Li and Agee, 2001; Halliday and Wood, 2009). Estimates are strongly influenced by the choice of oxygen fugacity in models (e.g., Chabot and Agee, 2003; Wood et al., 2008; Righter, 2011; Righter and Drake, 2003), and to some extent, melt composition and water content (Hillgren et al., 1996, Righter and Drake, 1997, Jaeger and Drake, 2000 Righter and Drake, 2003). Models of continuous equilibrium core formation at the base of a magma ocean can reproduce the mantle concentrations of a number of siderophile elements, including $\mathrm{Ni}$, Co, Mo, W, P, Mn, V, $\mathrm{Cr}, \mathrm{Ga}, \mathrm{Cu}, \mathrm{Pd}$ and $\mathrm{Nb}$ (Li and Agee, 2001; Wade and Wood, 2001; Wood et al., 2008, Righter, 2011). Some of these require that the composition of the forming Earth changed from reduced to more oxidised, similar to hetero- 
geneous accretion models (Wood et al., 2008). Magma ocean models resolve some aspects that had been problematic for earlier models. For example, settling of metals from a magma ocean removes the need for a mechanism for metals to percolate through solid mantle (Stevenson, 1990; Tonks and Melosh, 1992). These models continue to grow increasingly complex and more robust, and recent models include quantitative predictive expressions for up to 11 elements simultaneously (e.g., Righter, 2011).

Despite the successes of magma ocean models, they are not yet universally accepted. While experimental data have been extended to high pressures and temperatures, some of the scenarios described above envision physical conditions in a magma ocean that remain beyond the reach of current experimental techniques, and thus these models continue to rely on extrapolations. Predictions of partitioning of elements in a magma ocean involve a number of parameters and often rely on simplified thermodynamic equations, and can yield solutions that are non-unique (Righter and Drake, 2003). Furthermore, experimental data from other workers have suggested that even at extremely high pressures ( $\geq 60 \mathrm{GPa}$ ) partition coefficients of highly siderophile elements are not sufficiently reduced to be consistent with metal-silicate fractionation in a magma ocean (Ertel et al., 2006, Mann et al., 2012). These workers suggest that partition coefficients of highly siderophile elements at high pressures differ from one another by $0.5-3$ orders of magnitude, and thus the chondritic relative abundances of these elements in the mantle cannot be reproduced by metal-silicate differentiation (Mann et al., 2012).

\section{Core-mantle interaction}

Another alternative for the source of siderophile elements in the mantle that has received some attention is the possibility of exchange between the PGErich liquid outer core and the lower mantle at the base of mantle plumes (Walker et al., 1995, Brandon et al. 1998; Puchtel and Humayun, 2000, Brandon and Walker, 2005). Late-veneer models are partially based on the observation of near-chondritic PGE abundances in the mantle. Non- 


\section{Continuous core segregation during accretion}

A

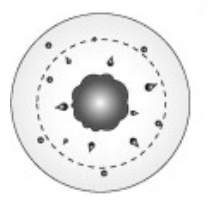

B

C

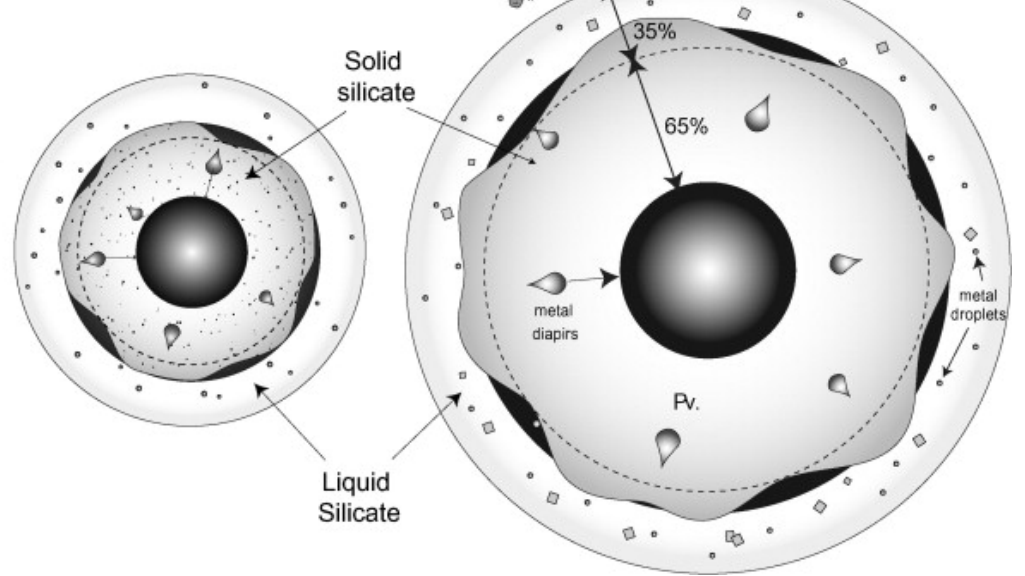

Figure 1.5: Schematic diagram of continuous core formation and metal-silicate equilibration in a magma ocean. Accreting metal is presumed to equilibrate with the mantle at the base of the magma ocean. The metal then passes diapirically through the lower mantle into the core. Figure from Wood et al. (2008).

chondritic PGE concentration ratios have been found in several studies of peridotites (Pattou et al., 1996, Snow and Schmidt, 1998). These suggested that if the relative abundances cannot be linked to a meteorite source, the most likely source for PGE is influx of material derived from the outer core and delivered into the upper mantle through mantle plumes (Pattou et al. 1996. Snow and Schmidt, 1998). However, this interpretation is not widely supported, and other PGE workers suggest that variations from chondritic relative abundances may be an artefact of within-mantle processes such as melt extraction and mantle metasomatism Rehkämper et al., 1999a; Handler and Bennett, 1999, Alard et al., 2000; Luguet et al., 2001).

\subsection{Meteorite samples}

Meteorites provide our only direct samples of the deep interior of planets and of primitive materials from the early solar system. As such, they are 
invaluable in the study of early Solar System processes. A number of meteorite samples are measured as part of this study to investigate Pt stable isotope variations in different types of meteorites, and to constrain the isotopic compositions of different reservoirs that play a role in models of Earth's differentiation, as described in the previous section. This section outlines classification scheme used to group meteorites, and provides additional detail for those meteorite groups that are used in this study. Information specific to the samples used in this study is provided in Appendix A.

\subsubsection{Classification of Meteorites}

The goal of all classification schemes is to group meteorite specimens that share common properties and, ideally, genetic relationships. Various classification schemes for meteorites have been proposed and continue to evolve over time with new data and new specimens. In the classification scheme of Krot et al. (2007, Fig. 1.6), meteorites are separated into two broad groups on the basis of their bulk composition and textures - chondrites and nonchondrites - which are further subdivided based on chemistry, mineralogy, petrography and oxygen isotope systematics. These groupings and the classification hierarchy are summarised in Fig. 1.6. An order called a clan is also included in this classification scheme, which represents groups that have petrogenetic relationships but have other characteristics that challenge group relationships (Krot et al., 2007).

Chondrites are undifferentiated meteorites that condensed directly from the molecular cloud from which the solar system formed, and have been subjected to little or no modification since by melting or differentiation of their parent bodies. They represent the most primitive solar system materials, and have elemental compositions similar to that of the solar photosphere. Some non-chondrites are compositionally similar to chondrites, retaining relatively high concentrations of metal and siderophile elements, but their textures show evidence of metamorphism and/or low degrees of partial melting. These are known as primitive achondrites, and are thought to have come 


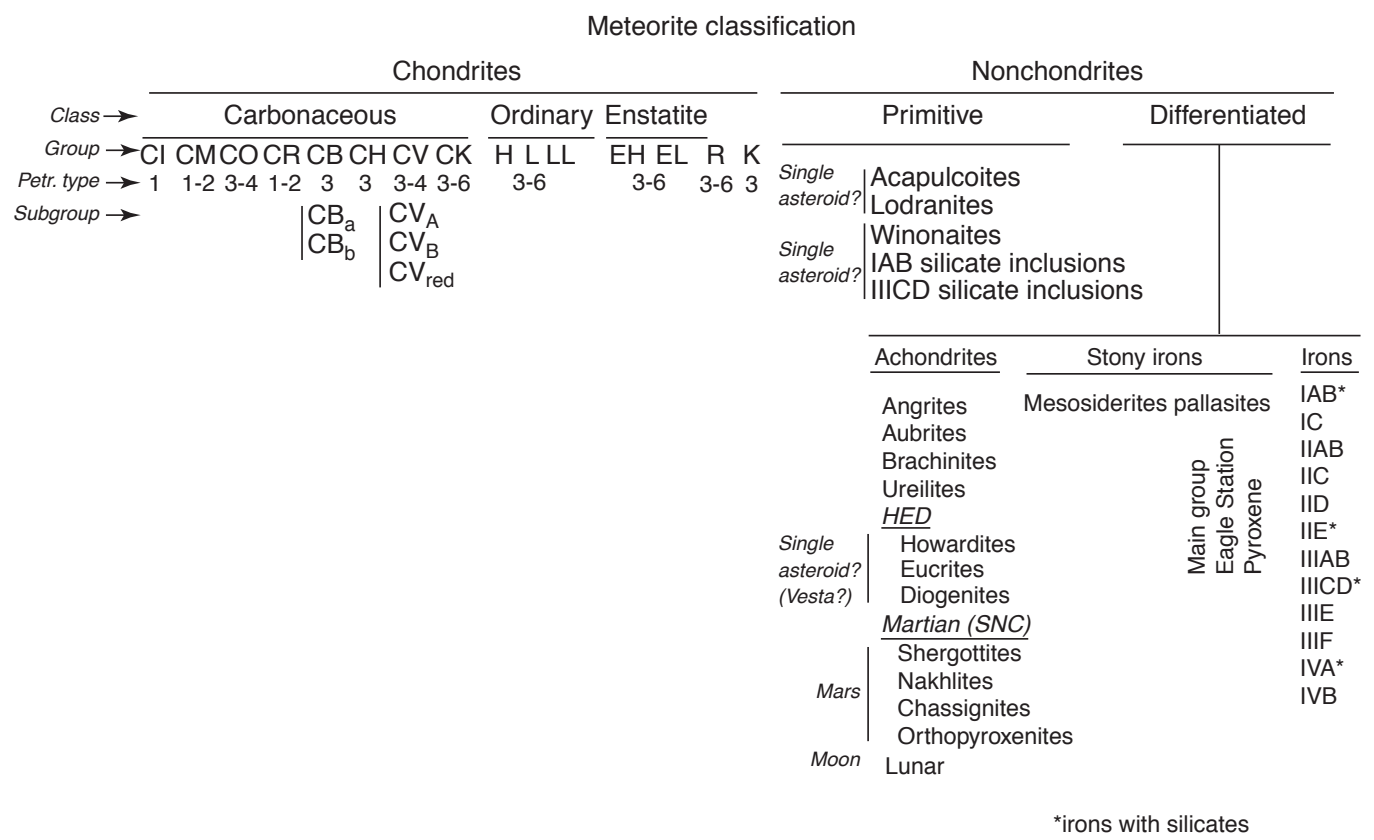

Figure 1.6: Meteorite classification scheme from Krot et al. (2007).

from parent bodies that underwent a lesser degree of melting, and thus did not fully differentiate (Krot et al. 2007). Achondrites are igneous products of crust-mantle differentiation on differentiated asteroid parent bodies. They have compositions that are highly fractionated from those of primitive solar system material, and different meteorites sample different parts of the silicate portions of their respective parent bodies. Iron meteorites consist largely of iron-nickel alloy, and originate from the cores of asteroidal parent bodies. Stony-iron meteorites contain roughly equal amounts of metals and silicates, and are thought to originate from the core-mantle boundary region of small planetesimals. Further detail on the classification of specimens within these groups follows, focusing primarily on those groups that were utilised in this study. Further details on the classification and the specific characteristics of the various classes of meteorites can be found in Krot et al. (2007), Weisberg et al. (2006), Scott and Krot (2007), Mittlefehldt (2007), Haack and McCoy (2007), Goldstein et al. (2009) and references therein. 


\subsubsection{Chondrites}

The primary classification of chondrites is based on their petrology (mineralogy and texture) and bulk chemical and oxygen isotopic compositions, although additional characteristics are sometimes used. Currently there are 15 groups of chondrites recognised, 13 of which comprise the three major classes of chondrites - carbonaceous, ordinary and enstatite chondrites. A number of chondrite specimens do not fit into any existing group, and are known as ungrouped chondrites. The primary classification is denoted by the letters in each group name (e.g. CV, LL, etc.), which are discussed further below. Chemical groupings are made on the basis of the relative abundances of lithophile (Fig. 1.7) and siderophile elements. A secondary classification scheme groups chondrites into petrologic types $1-6$, which are appended to the group name. Petrological types are intended to provide a guide to the nature and extent of asteroidal processing, although this classification is not rigorous. Types 1 and 2 describe the amount of aqueous alteration of the meteorite, with type 1 being more altered. Type 3 chondrites are thought to be the least altered and metamorphosed chondrites. Types 4-6 are thought to represent an isochemical metamorphic sequence, with increasing numbers representing increasing degrees of petrologic equilibration and recrystallisation (Weisberg et al., 2006, Krot et al., 2007).

\section{Carbonaceous chondrites}

Carbonaceous chondrites consist of eight different groups - CI, CM, CR, CO, $\mathrm{CV}, \mathrm{CK}, \mathrm{CB}$ and $\mathrm{CH}$. With the exception of the $\mathrm{CH}$ group, the second letter refers to the type meteorite for that group (e.g., Vigarano for CV; Table 1.2). In the $\mathrm{CH}$ group, the $\mathrm{H}$ refers to the high metal abundances in that group (Table 1.2). Each of the groups has specific properties, which are discussed in detail by Krot et al. (2007), Scott and Krot (2007) and Weisberg et al. (2006).

Carbonaceous chondrites are characterised by high abundances of refract- 


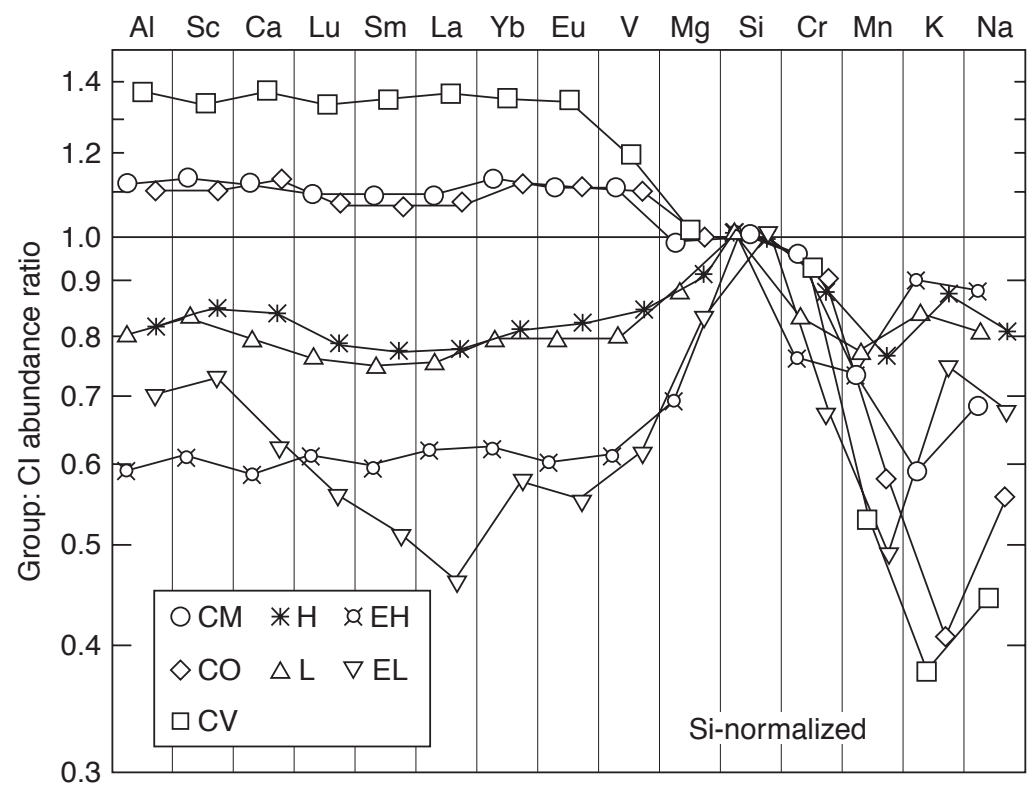

Figure 1.7: Lithophile element abundances in seven chondrite groups normalised to $\mathrm{Cl}$ chondrites and silicon, arranged in order of increasing volatility. Refractory elements are enriched in carbonaceous chondrites and depleted in ordinary and enstatite chondrites. Volatile elements are all depleted relative to $\mathrm{Cl}$ chondritelron meteorites

ory lithophile elements (Fig. 1.7) and oxygen isotope compositions that form a separate, mass independent trend that plots below the terrestrial fractionation line in three oxygen isotope space (Fig. 1.8). They show a much greater range in oxygen isotopic compositions than the ordinary or enstatite chondrites (Fig. 1.8). The carbonaceous chondrites contain variable amounts (ca. $0.1-13 \%$ by volume) of refractory material (Ca-Al-rich inclusions (CAI), amoeboid olivine aggregates (AOA)). CAI represent high-temperature condensates from the solar nebula and are the oldest dated materials that are thought to have formed in the early Solar System (Amelin et al. 2002). As such, CAI from carbonaceous chondrites have played an important role in establishing the chronology of the early solar system. CI chondrites are also notable as they provide best match in elemental abundances (especially volatile elements) to the solar photosphere (Anders and Grevesse, 1989: Grevesse and Sauval, 1998), which has led to them being considered the most primitive 


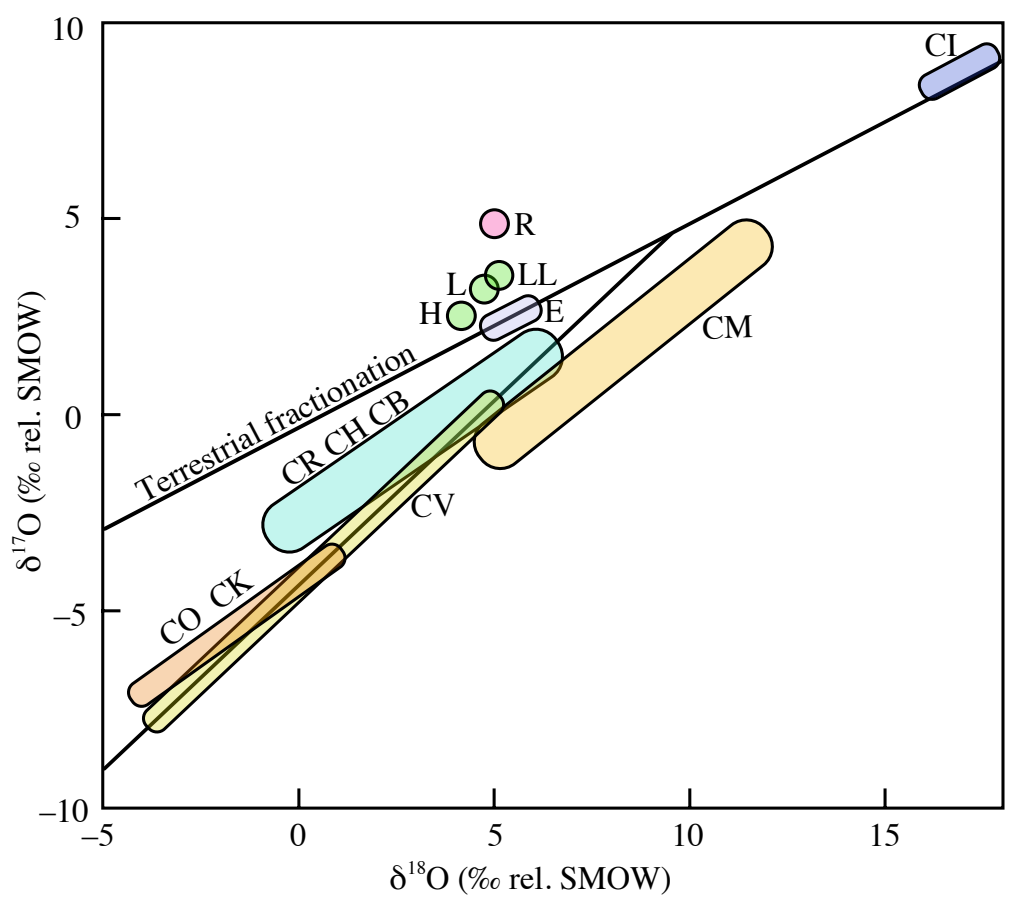

Figure 1.8: Schematic representation of the location and ranges of whole-rock isotopic compositions of major chondrite classes. Mass-dependent fractionation of oxygen isotopes produces a slope $=0.52$, as observed in Earth and Lunar samples which fall on the terrestrial fractionation (TF) line. The carbonaceous chondrite anhydrous mineral (CCAM) reference line is defined by mass-independent fractionations observed in $\mathrm{Ca}-\mathrm{Al}$-rich inclusions from the Allende carbonaceous chondrite meteorite. Figure modified from Clayton (2003).

meteorite samples available. However, the CI chondrites have been subjected to extensive, multi-stage aqueous alteration on their parent asteroid(s), which have erased their primary mineralogy and petrography McSween. 1979 Endress and Bischoff, 1993, 1996; Endress et al., 1996).

Seven carbonaceous chondrites were analysed in this study. Information on these specimens can be found in Appendix A

\section{Enstatite chondrites}

Enstatite chondrites have a unique mineralogy, including a variety of unusual sulphide, metal and nitride phases (Keil, 1968, Weisberg et al., 2006), indicating they formed under highly reducing conditions (Weisberg et al. 


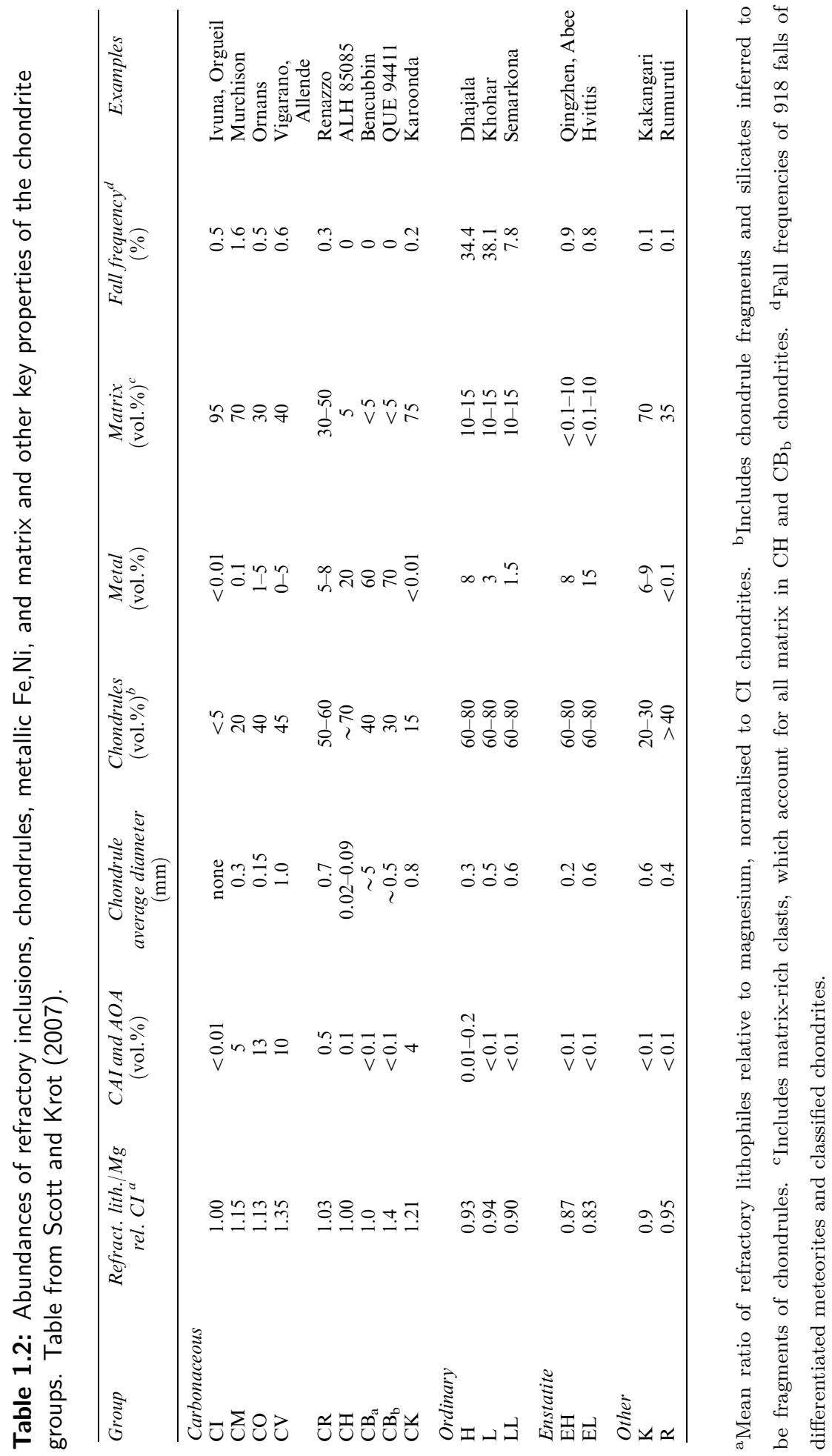


2006, Krot et al. 2007). They contain high abundances of cryptocrystalline, enstatite-rich chondrules (60-80 volume \%; Table 1.2), from which they derive their name. Enstatite chondrites are subdivided into EH and EL groups on the basis of metallic iron content: the high-Fe and high siderophile EH group, and the low-Fe and low siderophile EL group (Sears et al., 1982). The EH and EL groups are clearly resolved on the basis of mineralogy and bulk chemistry. The EH chondrites are more reduced than EL chondrites, and contain niningerite $((\mathrm{Mg}, \mathrm{Fe}, \mathrm{Mn}) \mathrm{S})$ and alkali sulphides, whereas the EL group is characterised by the presence of alabandite $((\mathrm{Mn}, \mathrm{Fe}) \mathrm{S})$ (Weisberg et al., 2006, Krot et al., 2007). Other systematics (e.g. degree of shock metamorphism; Rubin et al., 1997) are also used in the classification of enstatite chondrites. Although there is some overlap in cosmic ray exposure (CRE) ages from the two groups (EH: 0.5-7 Ma; EL: 4-18 Ma), the two distinct enstatite chondrite groups are considered to be distinct, representing two separate bodies that formed at similar distances from the Sun (Sears et al. 1982).

The enstatite chondrites are the only group with oxygen isotope compositions that plot along the terrestrial fractionation line, similar to Earth and the Moon. While the enstatite chondrites have significant chemical dissimilarities to Earth, isotopic similarities between Earth and enstatite chondrites have been found in number of other isotope systems (N, Mo, Ru, Os and Ti; Javoy et al., 2010, and references therein). It has been suggested that these similarities are evidence that the main building material for Earth was similar in composition to enstatite chondrites (e.g. Javoy, 1995, Javoy et al. 2010). However, this is not widely accepted, and mass balance equations based on Si isotope offsets between enstatite chondrites and the silicate Earth require unfeasibly large amounts of $\mathrm{Si}$ (ca. 26\%) to have been partitioned into Earth's core during core formation (Fitoussi and Bourdon, 2012, Savage and Moynier, 2013).

One enstatite chondrite was analysed in this study. Specimen details can be found in Appendix A. 


\section{Ordinary chondrites}

Ordinary chondrites are the by far the most commonly found meteorites on Earth (ca. 80\% of all meteorite falls; Krot et al., 2007). They include include the LL, L and H groups, where the letters indicate increasing metal and total iron content. Ordinary chondrites are characterised by a high abundance of large (mm-scale) chondrules, low matrix abundance, and rare refractory inclusions (Table 1.2, Weisberg et al., 2006). The ordinary chondrites have oxygen isotope compositions that plot above the terrestrial fractionation line (Fig. 1.8), and they are relatively depleted in refractory lithophile elements (Fig. 1.7). The ordinary chondrite groups have overlapping petrologic characteristics and their oxygen isotopic compositions define a common fractionation line, suggesting a single common source for the chondrules (Weisberg et al. 2006). The slight difference in the bulk isotopic composition may be related to size sorting, as chondrule sizes increase in the order $\mathrm{H}-\mathrm{L}-\mathrm{LL}$ (Rubin, 1989).

Three ordinary chondrites were analysed in this study. Information on these specimens can be found in Appendix A.

\section{$\mathrm{K}$ and $\mathrm{R}$ type chondrites}

The $\mathrm{R}$ chondrite (Rumuruti-like) group and the K (Kakangari-like) grouplet differ from the other classes of chondrites in their mineralogy and their oxygen isotope compositions, and are thought to represent independent groups of meteorites (Krot et al., 2007). The K chondrites have O isotope compositions similar to carbonaceous chondrites, are highly reduced like enstatite chondrites, and have refractory lithophile abundances similar to ordinary chondrites. $\mathrm{R}$ chondrites have a number of properties in common with ordinary chondrites, including types of chondrules, few refractory inclusions and similar chemical compositions for most elements. However, they have a higher matrix content (Table 1.2), are more oxidised, and have higher ${ }^{17} \mathrm{O} /{ }^{16} \mathrm{O}$ ratios than the ordinary chondrites (Fig. 1.8, Krot et al. 2007). 
No K or R type chondrites were analysed in this study.

\section{Oxidation state of chondritic meteorites}

A number of other secondary classification parameters for chondrite meteorites exist (Krot et al., 2007; Weisberg et al., 2006), which are not detailed here. However, stable isotopic fractionations may be related to oxidation conditions, and therefore a brief discussion of the oxidation states of chondrites follows.

A wide range of oxidation states are recorded by the different chondrite groups. The role of nebular and asteroidal processes in the oxidation states of chondrites is controversial, and the oxidation state of each group does not necessarily reflect that of its individual components (e.g., all CAI formed under reducing conditions; Krot et al., 2007). The oxidation state of a particular group is determined by the distribution of iron amongst its three common oxidation states (0 in FeNi-metal and Fesulphides, +2 in silicates and +3 in oxides), which are represented in a Urey-Craig diagram (Fig. 1.9). Generally, oxidation states in chondrites increase in the order enstatite- $\mathrm{K}$-ordinary-carbonaceous $+\mathrm{R}$ chondrite classes (Fig. 1.9). Within the ordinary chondrites, oxidation state increases (and siderophile element abundances decrease) from $\mathrm{H}$ to $\mathrm{L}$ to LL (Krot et al. 2007). The oxidation state of carbonaceous chondrites increases in the order $\mathrm{CB}-\mathrm{CH}-\mathrm{CR}-\mathrm{CO}-\mathrm{CV}-\mathrm{CK}-\mathrm{CM}-\mathrm{CI}$.

\subsubsection{Non-chondrites}

Aside from meteorites from Mars and the Moon, the non-chondrites represent a number of small planetesimals that formed within the first few Myr of the Solar System. Some of the planetesimals only experienced minor degrees of melting, the so-called 'primitive achondrites'. Primitive achondrites have retained metal and siderophile element abundances similar to chondrites and have not isotopically re-equilibrated, yet they have metamorphic and/or ig- 


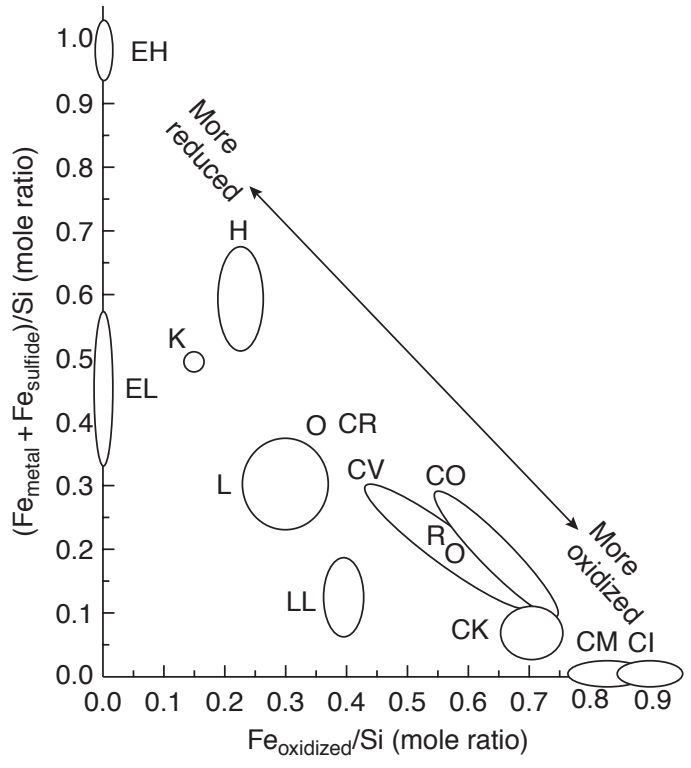

Figure 1.9: Urey-Craig diagram showing relative iron contents and oxidation states of the different chondrite groups. Figure from Krot et al. (2007).

neous textures. Other planetesimals experienced planetesimal-wide melting and differentiation, forming $\mathrm{Fe}-\mathrm{Ni}$ cores and silicate mantles and crusts. Differentiated non-chondrites sample all parts of these bodies, from their cores (iron meteorites) and core-mantle boundaries (stony-irons) to their mantles and crusts (achondrites).

\section{Iron meteorites}

Iron meteorites are largely made up of the $\mathrm{Fe}-\mathrm{Ni}$ alloys kamacite and taenite. They may also contain various other alloys as well as accessory sulphides, carbides, nitrides, phosphides, oxides and phosphates and, in some cases, substantial amounts of silicate (Haack and McCoy, 2007; Goldstein et al. 2009). Iron meteorites are classified on the basis of their structural and chemical properties. Structural assessments are based on the appearance of polished sections that have been etched with acid, which reveals structures such as the characteristic Widmanstätten patterns shown in Fig. 1.10A, that differ depending on the relative abundances of different $\mathrm{Fe}-\mathrm{Ni}$ alloys 
(i.e., nickel content). They are divided into hexahedrites $(\mathrm{H}$; low $\mathrm{Ni}$, no Widmanstätten patterns), octahedrites (O; with Widmanstätten patterns, sub-classified according to lamellae width) and ataxites (D; high Ni content, no Widmanstätten patterns). Octahedrites are further subdivided on the basis of kamacite lamellae width as follows: Ogg — coarsest, bandwidth $>3.3 \mathrm{~mm}$; Og — coarse, $1.3-3.3 \mathrm{~mm}$; Om - medium, 0.5-1.3 mm; Of fine, $0.2-0.5 \mathrm{~mm}$; Off - finest, $<0.2 \mathrm{~mm}$. The kamacite bandwidth decreases with increasing $\mathrm{Ni}$ content, and relates to the cooling rate of the planetary cores that they sample (Haack and McCoy, 2007; Goldstein et al., 2009).

Chemical classification schemes are based on the proportions of welldetermined trace elements (particularly Ga, Ge and Ir) versus nickel content. Plots of these elements (e.g., Fig 1.10B) reveal distinct groupings, and on the basis of these iron meteorites are divided into 13 chemical groups that are designated by combinations of roman numerals and letters. Each of these groups is thought to represent a distinct parent body, however about $15 \%$ of all known iron meteorites do not belong to any of the chemical groups. Between the grouped and ungrouped types, iron meteorites are thought to sample between 30 and 50 separate asteroidal parent bodies (Burbine et al. 2002, Haack and McCoy, 2007).

Seven iron meteorites were analysed in this study. Information on these specimens can be found in Appendix A.

\section{Stony irons}

Stony iron meteorites include the mesosiderites and pallasites. Pallasites are the most abundant group of stony irons, and contain variable amounts of silicate (mostly olivine) suspended in Fe-Ni metal. Pallasites are thought to sample the core-mantle boundaries of differentiated asteroid parent bodies (Burbine et al., 2002; Haack and McCoy, 2007). Mesosiderites are breccias of silicate and metal clasts with irregular textures, thought to have formed by impact mixing of a core fragment on the surface of a basaltic (i.e. differentiated) asteroid (Burbine et al., 2002), possibly followed by collisional 

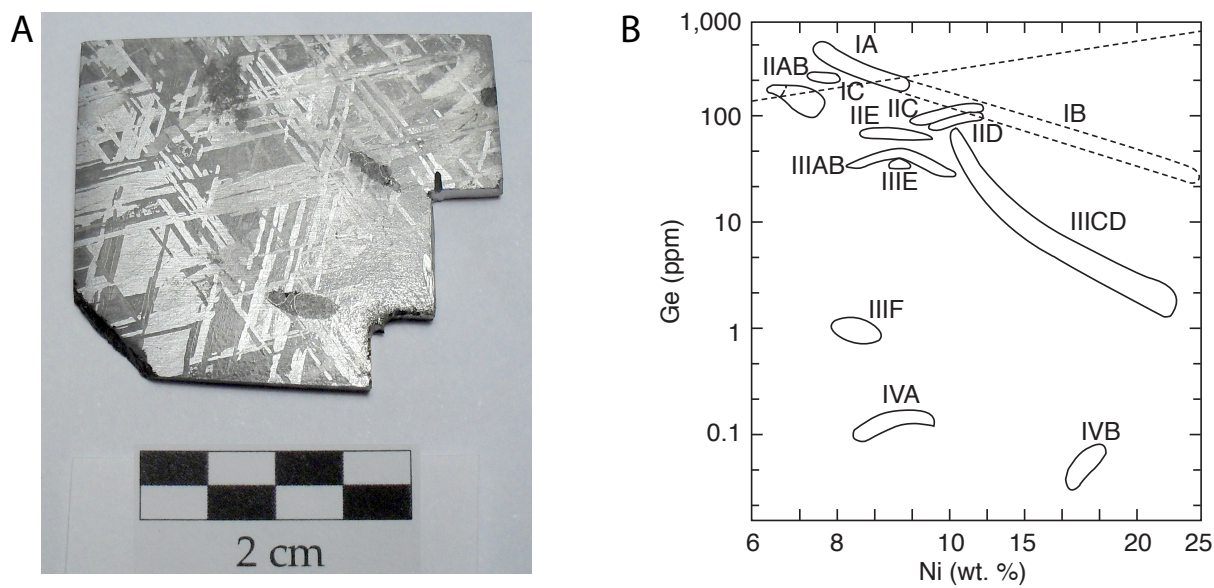

Figure 1.10: Structural and chemical classification of iron meteorites. A) Photograph of a specimen of Gibeon, a type IVA iron meteorite, that has been etched to show the Widmanstätten patterns, which are characteristic of iron meteorites within the octahedrite structural classification. This specimen also shows several dark blobs, which are troilite (FeS) inclusions. B) An example of chemical classification of iron meteorites, using a plot of $\mathrm{Ni}$ vs. $\mathrm{Ge}$. This plot, taken from Krot et al. (2007), shows the fields for the 13 chemical groupings of iron meteorites.

disruption and reassembly of the asteroid (Rubin and Mittlefehldt, 1993).

No stony-iron meteorite samples were used in this study.

\section{Achondrites}

The achondrites are igneous products of silicate differentiation on asteroidal parent bodies. They include angrites, aubrites, brachinites, ureilites and the howardite-eucrite-diogenite (HED) clan, as well as Martian and Lunar meteorites (Mittlefehldt, 2007). Achondrites sample the silicate mantles and crusts of differentiated asteroids, with each group thought to represent a distinct parent body. Ureilites were the only achondrite group sampled in this study, so further detail is only provided for that group.

Ureilites comprise the second largest group of achondrites. They exhibit features of both primitive and differentiated achondrites, and are thought to represent the mantle of an asteroid that only partially melted. Lithophile element contents of ureilites are highly fractionated from chondritic abund- 
ances and they are depleted in incompatible lithophile elements (Al, Na and $\mathrm{K}$ ), which suggests they are highly fractionated igneous rocks (Mittlefehldt, 2007, Rankenburg et al., 2008). However, they are enriched in refractory siderophiles and contain noble gas abundances that are difficult to reconcile with igneous processing. Ureilites also show extreme oxygen isotope heterogeneity, with compositions parallel to the carbonaceous chondrite anhydrous mineral (CCAM) line (Clayton and Mayeda, 1988). These heterogeneities would be expected to be erased if the ureilite parent body had undergone extensive melting and re-equilibration. Ureilites have ultramafic compositions, but the modal abundances of the major silicate minerals vary significantly amongst members of the class. Ureilites exhibit widely varying degrees of shock, but most are unbrecciated or monomict (Mittlefehldt, 2007). Some polymict ureilites contain a large variety of other meteoritic material that is distinct from monomict ureilites (Guan and Crozaz, 2001), which is taken as evidence for impact events on the ureilite parent body Goodrich et al. 2004). Ureilites contain up to $10 \%$ interstitial material that is rich in elemental carbon in the form of graphite or nano-diamonds, which may have formed as a result of impacts (Mittlefehldt, 2007). While a number of models for the genesis of ureilites have been considered (a review is provided by Goodrich, 1992), no model yet proposed can explain all of the features of ureilite samples.

One ureilite was used in this study. Specimen information can be found in Appendix A.

\section{Primitive achondrites}

Primitive achondrites have bulk chemical compositions similar to chondrites, but have achondritic textures. Primitive achondrites are thought to represent either metamorphosed chondrites or residues from a small degree of partial melting (McCoy et al., 1997a b; Krot et al., 2007). In the classification scheme of Krot et al. (2007), the primitive achondrites consist of the acapulcoites, lodranites and winonaites, with potential links to groups from other classes. 
Acapulcoites and lodranites have similar oxygen isotope compositions (Clayton et al., 1992), as well as similar mineralogies, mineral compositions, thermal histories and cosmic ray exposure ages (McCoy et al., 1997b), which suggest they are derived from a common parent body, and they are collectively known as the acapulcoite-lodranite clan. Acapulcoites have chondritic bulk chemical compositions, and some contain relict chondrules $(\overline{\mathrm{Ru}}-$ bin, 2007). Lodranites are distinctly non-chondritic, with a modal mineralogy that suggests extraction of a basaltic component (Mittlefehldt, 2007). Average bulk compositions of the acapulcoite-lodranite clan are similar to ordinary chondrites, and they are widely considered to have formed by partial melting and/or extensive high temperature anatexis of primitive, reduced chondritic material (Rubin, 2007; Mittlefehldt, 2007)

Winonaites also have similar mineralogy and chemistry to acapulcoites and lodranites, but have distinct oxygen isotopic compositions indicating they sample a different parent body (Burbine et al., 2002). Silicate inclusions in IAB and IICD iron meteorites have similar mineralogy and composition to chondrites but have non-chondritic recrystallised textures, and these have been linked to winonaites through similarities in oxygen isotope compositions (Clayton and Mayeda, 1996). There is substantial heterogeneity in the chemical and textural compositions within this group, and the mixture of primitive silicate material with iron provides a considerable challenge to explain the origin of the winonaite-IAB-iron silicate inclusion clan. Current petrogenetic models for this group suggest a chondritic precursor material that underwent metamorphism, partial melting and incomplete differentiation, potentially followed by catastrophic impact breakup and reassembly of the parent body (Benedix et al., 1998, 2000).

Four primitive achondrites - two acapulcoites and two winonaites - were used in this study. Information on these specimens can be found in Appendix A. 


\subsection{Non-traditional stable isotope systems}

The potential of stable isotopes for investigating geological processes has long been recognised (e.g., Nier and Gulbransen, 1939, Dole and Slobod, 1940, Urey, 1947, 1948). Until relatively recently, the practical measurement of stable isotopes was limited to light elements such as H, C, N, O and S, which can be readily measured in a gaseous form. The field of 'non-traditional' (i.e., elements other than those listed above) stable isotope geochemistry has advanced rapidly over the past two decades, particularly since the advent of multiple collector inductively coupled plasma mass spectrometers (MC-ICPMS). Improvements in these instruments and in measurement techniques have allowed progressively smaller natural variations to be precisely and accurately determined. The list of non-traditional stable isotope systems that have now been measured in natural samples is growing increasingly long (e.g., Li, Mg, Si, Cl, Ca, Cr, Fe, Cu, Zn, Se, Mo, Hg and Tl), and many of these have provided valuable new insights in the earth, environmental and planetary sciences (e.g. Halliday et al., 1998, Maréchal et al., 1999, Zhu et al. 2000; Belshaw et al., 1998, 2000; Beard and Johnson, 1999; Young and Galy, 2004, Anbar and Rouxel, 2007; Fitoussi et al., 2009).

The following section presents brief discussions of four non-traditional stable isotope systems that have been developed in recent years: Fe, Si, $\mathrm{Cr}$ and Ni. This review is intended to be illustrative of how other stable isotope systems have been applied to similar problems as those considered here, rather than a comprehensive account of the uses of these isotopic systems, and necessarily omits a great deal of important work in these fields. It focuses particularly on mass-dependent fractionations by high temperature (especially planetary) processes. A brief discussion of the Pt stable isotope system is then presented in this context.

Note: this section makes use of delta $(\delta)$ notation, which expresses the parts-per-thousand (i.e., per mil; \%o) difference in a measured isotope ratio 
in a sample compared with the same ratio in a standard:

$$
\delta=\left[\frac{R_{\text {sample }}}{R_{\text {standard }}}-1\right] \times 1000
$$

where $\mathrm{R}$ is a ratio of two isotopes of the element of interest. In studies of mass-dependent isotopic fractionation, it is also common to express fractionations in terms of delta units divided by the difference in mass between the numerator and denominator isotope (i.e., \%o $\mathrm{amu}^{-1}$ ), and these are generally used in the discussion below. The isotopic standard used varies between isotope systems and sometimes amongst different workers within the same isotopic system. For the purposes of this discussion the standard used is generally omitted, and data comparisons used are taken from literature sources where inter-calibrations between standards have already been taken in to account.

Dauphas and Rouxel (2006) describe three main processes that can produce isotopic fractionations at high temperatures in natural systems: 1) diffusion in liquids, solids or gases; 2) evaporation and condensation; 3) phase partitioning. The magnitude of equilibrium isotopic fractionations is temperature dependent (providing the basis for stable isotope geothermometers), and predicted to decrease with increasing temperatures. Thus, high temperature equilibrium isotopic fractions are likely to require highly precise measurements to be resolved. Non-equilibrium high temperature isotopic fractionations have received increasing attention recently, and resolvable fractionations in high-temperature processes have now been measured in a range of isotope systems (Richter et al., 2009). Recent theory, experiments, and meteorite measurements suggest high-temperature isotopic fractionations can result from bonding differences between silicate and metallic liquids (Polyakov, 2009). 


\subsubsection{Iron stable isotopes}

Iron ( $\mathrm{Fe}$ ) is the most abundant element on Earth, comprising $32 \%$ of Earth's overall mass and $85.5 \%$ of its core (McDonough, 2003). Despite the concentration of $\mathrm{Fe}$ in the core, Fe is still a major constituent of the silicate Earth, making up ca. $6.3 \%$ of the mass of the mantle, and is the fourth most abundant element in the crust. Fe has four naturally occurring stable isotopes $\left({ }^{54} \mathrm{Fe},{ }^{56} \mathrm{Fe},{ }^{57} \mathrm{Fe}\right.$ and $\left.{ }^{58} \mathrm{Fe}\right)$, and can exist in a range of oxidation states, but commonly occurs in nature as Fe(II) and Fe(III).

In a seminal high-precision Fe stable isotope study, Beard and Johnson (1999) found that a range of terrestrial igneous rocks have approximately homogeneous Fe isotopic compositions, and that the silicate Earth is enriched in heavy isotopes of Fe by $0.039 \pm 0.008 \%$ amu $\mathrm{am}^{-1}$ compared to chondrites (Beard and Johnson, 1999, Fig. 1.11). This finding has been supported by a number of subsequent studies (Beard et al., 2003; Rouxel et al., 2003, Dauphas et al. 2004b c; Poitrasson et al., 2004, Williams et al., 2004). Zhu et al. (2001) found that the Fe isotopic compositions of a range of terrestrial and meteoritic samples plot on a mass-dependent fractionation line, suggesting that the solar nebula was isotopically homogeneous for Fe, and was subsequently fractionated in a mass-dependent manner either before or during the formation of chondrules and asteroidal parent bodies. Vesta and Mars are isotopically lighter than Earth, and have approximately the same isotopic composition as chondrites and the IRMM-14 iron isotope standard (Poitrasson et al., 2004). Poitrasson et al. (2004) found that Lunar samples were relatively enriched in heavy isotopes compared to chondrites and to Earth $(0.069 \pm 0.010 \%$ o $\mathrm{amu}^{-1}$ ). However, Weyer et al. (2005) suggested that the heavy lunar mantle Fe isotope signature found by (Poitrasson et al., 2004) arose from analysing predominantly high-Ti basalts, and showed that picritic and other high-Mg lunar basalts had Fe isotopic compositions very similar to terrestrial basalts. Lunar regolith is found to be relatively enriched in the heavy isotopes of iron, and these enrichments correlate with soil maturity index, suggesting that exposure to 'space weathering' on the Lunar surface results in vaporisation of 
Fe (and other elements), which preferentially removes the lighter isotopes (Wiesli et al., 2003; Moynier et al., 2006).

The cause of isotopically heavy composition of Earth and the Moon has been debated. Core-mantle differentiation is not considered to provide an adequate explanation, as metals from pallasite meteorites have been shown to be isotopically heavier than the coexisting silicate phases (Zhu et al. 2001; Mullane et al., 2002; Kehm et al., 2003; Poitrasson et al., 2005), which implies the silicate Earth should be isotopically lighter than the core. However, recent estimates of equilibrium Fe isotope fractionation factors suggest that the direction of the fractionation may be reversed at ultra-high pressures, and that the heavy Fe isotopic composition of the PUM indicates that silicate-metal differentiation occurred under high pressure conditions (Polyakov, 2009). This scenario also requires that core formation was essentially complete before the moon-forming giant impact (Polyakov, 2009). Poitrasson et al. (2004) suggested that the enrichment in heavy isotopes of the Earth and Moon was the result of evaporative loss of Fe during the Moon forming impact, although, to fit other isotope constraints, they further argue that the loss of iron was primarily from the metal cores of the planets, which should show related effects in other isotope systems (Dauphas and Rouxel, 2006). Another possibility is that the enrichment of heavy Fe isotopes in terrestrial and Lunar samples may be related to the oxidation state of iron, and that during core-mantle differentiation isotopically heavy ferric iron $\left(\mathrm{Fe}^{3+}\right)$ was preferentially released from the proto-mantle into a magma ocean (leaving the mantle enriched in ferrous iron, $\mathrm{Fe}^{2+}$ ), while isotopically light native iron $\left(\mathrm{Fe}^{0}\right)$ was preferentially incorporated into the core (Williams et al., 2006).

Iron meteorites from all groups are also enriched in heavy Fe relative to chondrites by $0.032 \pm 0.010 \% \mathrm{amu}^{-1}$ (Zhu et al., 2001, Kehm et al., 2003 . Poitrasson et al. 2005). Measurements of bulk iron meteorites may be complicated by fractionations between Fe-Ni alloy (kamacite and taenite) phases (Zhu et al., 2002; Poitrasson et al., 2005; Horn et al., 2006, Weyer et al. 


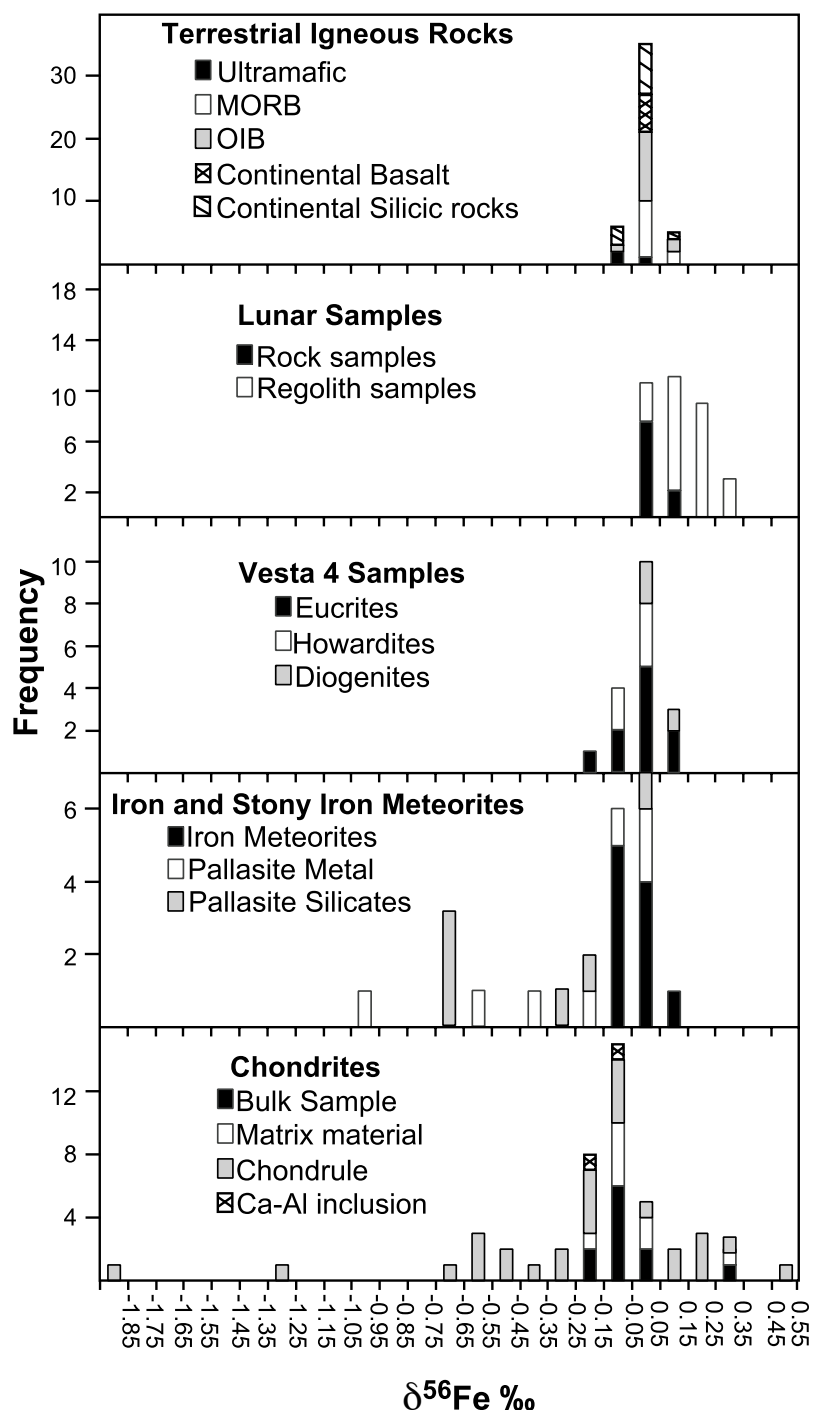

Figure 1.11: Frequency diagram of Fe isotope compositions measured in terrestrial and extraterrestrial samples. The bin range is $0.1 \% 0$ in $\delta^{56} \mathrm{Fe}$, which is typical of the external precision of the measurements. Figure is from Beard and Johnson (2004), and includes data from a number of sources (Beard et al., 2003 Wiesli et al. 2003 Zhu et al. 2001: Mullane et al. 2002 2003a b c Kehm et al. 2003) corrected for inter-laboratory bias and choice of standards. Data have been renormalised such that zero is centred around the composition of terrestrial igneous rocks. Note that the $\delta^{56} \mathrm{Fe}$ notation represents the \%o variation in the ${ }^{56} \mathrm{Fe} /{ }^{54} \mathrm{Fe}$ ratio of the sample relative to the IRMM-014 standard and thus variations in this figure must be divided by two to be compared with those described in the text, which are expressed in $\% \mathrm{amu}^{-1}$. 
2007), and between metals and phosphide (schreibersite) and sulphide (troilite) inclusions (Williams et al., 2006). Yet, Fe isotope compositions reported from bulk sampling of iron meteorites generally reveal homogeneous compositions (Fig. 1.11) (Zhu et al., 2001; Kehm et al., 2003; Williams et al. 2006).

\subsubsection{Silicon stable isotopes}

Silicon ( $\mathrm{Si}$ ) is the third most abundant element on Earth, comprising 16.1\% of Earth's mass (McDonough, 2003), and the second most abundant element in the crust (ca. $28 \%$ by mass). Si has three stable isotopes: ${ }^{28} \mathrm{Si},{ }^{29} \mathrm{Si}$ and ${ }^{30} \mathrm{Si}$.

Mass independent stable $\mathrm{Si}$ isotopic fractionations have been used to investigate the stellar environment and astrophysical setting of the forming Solar System. Silicon isotope compositions measured in pre-solar SiC grains show exotic compositions that are related to their nucleosynthetic production in stars. As such, stable isotopes of Si have played a role in constraining models of galactic chemical evolution (e.g., Nittler and Dauphas, 2006). Silicon isotopes could also have been fractionated by nebular processes such as evaporation and impact volatilisation. Large Si isotopic fractionations are observed in $\mathrm{Ca}-\mathrm{Al}-$ rich inclusions (CAI) and chondrules, which are explained by kinetic evaporation and condensation processes (Chakrabarti and Jacobsen, 2010). Based on Si isotope data, recent models of the evaporation of CAI under various conditions simulating the solar nebula suggest that CAI were molten on timescales of days to weeks (Shahar and Young, 2007), which places strong constraints on the timescales and mechanisms of the related heating event(s).

Mass-dependent fractionation of Si stable isotopes by planetary processes have been the focus of a number of studies, and Si isotope compositions have been measured in samples from Earth, the Moon, Mars and a range of primitive and differentiated meteorites (Molini-Velsko et al., 1986; Georg et al., 2007, Chakrabarti and Jacobsen, 2009, Ziegler et al., 2009, Fitoussi 
et al., 2009; Chakrabarti and Jacobsen, 2010; Ziegler et al., 2010; Savage et al. 2011; Armytage et al. 2011; Fitoussi and Bourdon, 2012; Armytage et al. 2012). While there has been some disagreement in the literature, most studies agree that the PUM has a non-chondritic Si isotopic composition, which is isotopically heavier than chondrites (Fig 1.12).

The most common explanation for this offset is that $\mathrm{Si}$ is one of the light elements in the core. Based on the density of Earth's core inferred from seismic studies, the core is thought to contain a significant fraction of elements lighter than Fe and Ni Birch, 1964; Poirier, 1994, McDonough, 2003. Li and Fei, 2007). The terrestrial mantle has Mg/Si ratios higher than found in chondrites, which has been used to argue that $\mathrm{Si}$ is the dominant light element in the core (Allègre et al., 1995; Palme and O'Neill, 2007; Wade and Wood, 2005). The removal of some silicon into the core is consistent with partitioning experiments, which show that Si becomes more siderophile under increasing temperature and pressure conditions and decreasing oxygen fugacity (Kilburn and Wood, 1997; Gessmann et al., 2001; Wade and Wood, 2005; Shahar et al., 2009, 2011). Theoretical fractionation calculations (Schauble et al., 2007) and natural isotopic fractionations observed between metals and silicates in some meteorites (Ziegler et al., 2009, 2010) also suggest that partitioning of some Si into the core should impart a measurable stable isotopic fractionation in the PUM, with isotopically lighter Si fractionating into the metallic core (Georg et al., 2007; Fitoussi et al., 2009). Using mass-balance equations, the isotopic offset between chondrites and PUM can be used to estimate the amount of Si present in the core. Based on the range of published offsets, calculated Si content in the core span a wide range (0 to $>20$ wt\%), but estimates of ca. 6-9 wt\% (e.g., Shahar et al., 2009; Armytage et al. 2011) are consistent with estimates derived using other chemical and physical constraints (McDonough, 2003, Wade and Wood, 2005).

Lunar rocks have an average $\mathrm{Si}$ isotopic composition of $\delta^{30} \mathrm{Si}=-0.29 \pm$ 0.08 Armytage et al. 2012), which is identical to the PUM value of Savage et al. (2011). However, samples from other differentiated bodies (including 


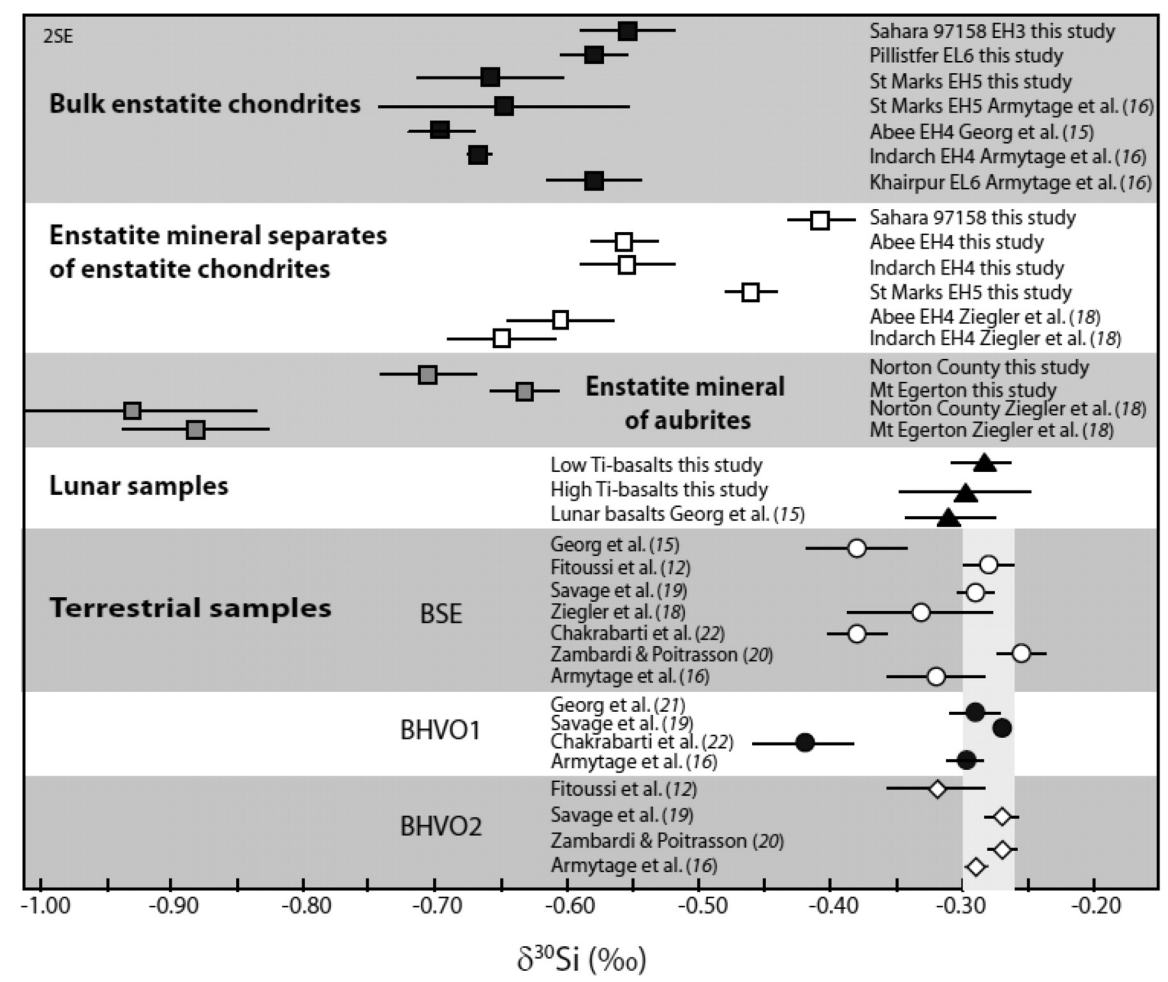

Figure 1.12: $\mathrm{Si}$ isotopic compositions of chondritic meteorite, lunar and terrestrial samples. The vertical band shows the mean BSE (i.e., PUM) value from Fitoussi et al. (2009). Figure taken from Fitoussi and Bourdon (2012), and annotations on the figure refer to references therein.

Mars and Vesta) have compositions identical to chondrites Armytage et al. 2011). The unique composition of the Earth is thought to reflect partitioning of Si into the core that only occurred under the comparatively high temperatures and pressures of core formation on Earth (Gessmann et al., 2001), and did not happen on the other smaller differentiated bodies sampled or, presumably, the putative Mars-sized Moon-forming impactor. Hydrodynamic simulations of Moon formation by a giant impact with a Mars sized body (ca. 0.1 Earth masses) suggest that most of the moon forming material should have derived from the impactor (Canup and Asphaug, 2001). One proposed explanation for the common Si isotopic compositions of Earth and the Moon is that the giant vapour cloud from which the Moon condensed had 
sufficient time to isotopically equilibrate with the proto-Earth in the aftermath of the Giant Impact (Pahlevan and Stevenson, 2007). However, it has recently been proposed that the Earth-Moon system could have lost angular momentum through a resonance between the Sun and the Moon Ćuk and Stewart, 2012), which potentially allows for a larger impactor (ca. 0.4 Earth masses) and results in the Moon forming disk being comprised primarily of material from Earth (Canup, 2012), providing another possible explaination for their isotopic similarities.

\subsubsection{Chromium stable isotopes}

Chromium (Cr) is the Earth's 10th most abundant element (McDonough, 2003) and has four naturally occurring stable isotopes: ${ }^{50} \mathrm{Cr},{ }^{52} \mathrm{Cr},{ }^{53} \mathrm{Cr}$ and ${ }^{54} \mathrm{Cr}$. It is a very important industrial metal with numerous applications. Chromium most commonly occurs naturally in the trivalent state, $\mathrm{Cr}$ (III), which is considered an essential nutrient, but can also exist in the hexavalent state, $\mathrm{Cr}(\mathrm{VI})$, which is an industrial pollutant that is known to be highly toxic. The mobility of $\mathrm{Cr}$ in the environment depends strongly on its redox state. Isotope ratio shifts have been found to occur during reduction reactions and, thus, the first applications of $\mathrm{Cr}$ stable isotope measurements were in tracing the reduction of $\mathrm{Cr}(\mathrm{VI})$ to $\mathrm{Cr}(\mathrm{III})$ in the environment Ellis et al. 2002, 2004, Johnson and Bullen, 2004). Cr stable isotopes have also been used recently to trace the oxidation history of the ocean and atmosphere over geological time scales (Frei et al., 2009).

Chromium is a compatible element in the mantle, and decreases in concentration from ultramafic igneous rocks (ca. $1000 \mathrm{ppm}$ ) to felsic rocks (ca. 20 ppm in granites; Faure, 1998). The first measurements of Cr stable isotope variations in terrestrial igneous rocks (including mantle xenoliths, ultramafic cumulates, oceanic and continental basalts, and Cr ores) were published by Schoenberg et al. (2008). These authors found that melting mantle rocks to form basalts, which under most conditions involves a change in oxidation state from $\mathrm{Cr}$ (III) to $\mathrm{Cr}$ (II) (Wood et al., 2008), does not cause any meas- 
urable $\mathrm{Cr}$ isotopic fractionation. The only significant $\mathrm{Cr}$ isotopic differences were found in crocoites, a lead chromate mineral, which were attributed to repeated redox cycling in hydrothermal processes. The limited variations in $\mathrm{Cr}$ stable isotope compositions in terrestrial silicates constrain the $\mathrm{Cr}$ isotopic composition of the PUM to within ca. $0.05 \% \mathrm{amu}^{-1}$ (Schoenberg et al. 2008).

Earth's mantle is found to be depleted in Cr compared to chondritic meteorites, which may suggest that $\mathrm{Cr}$ was partitioned into the core (Ringwood et al. 1990; Allègre et al., 1995, McDonough, 2003) or, given that $\mathrm{Cr}$ is a moderately volatile element, the depletion relative to chondrites may be related to evaporation/condensation processes (Drake et al., 1989). Moynier et al. (2011) measured Cr stable isotope compositions in a range of chondritic meteorites, and found that all classes of chondrites are isotopically lighter than PUM by up to $0.4 \% \mathrm{amu}^{-1}$ (Fig. 1.13). Based on mass balance calculations, they conclude that the difference in $\mathrm{Cr}$ isotopic compositions between silicate earth and chondrites is controlled by partitioning into Earth's core during core formation (Moynier et al., 2011). Isotope data from more volatile systems $(\mathrm{Zn}$ and $\mathrm{Cu}$ ) do not correlate with $\mathrm{Cr}$, suggesting that volatility does not play a role in the $\mathrm{Cr}$ isotopic fractionations. Moynier et al. (2011) also found that ordinary chondrites generally have heavier $\mathrm{Cr}$ isotope compositions than carbonaceous chondrites (Fig. 1.13), and that the isotopic compositions of carbonaceous chondrites broadly correlate with the abundance of refractory inclusions. These data suggest that the solar nebula contained at least two reservoirs with distinct $\mathrm{Cr}$ isotopic compositions, and that $\mathrm{Cr}$ stable isotopes may be useful to place constraints on mixing in the solar nebula and chondrule formation (McDonough, 2011).

\subsubsection{Nickel stable isotopes}

Nickel (Ni) has five stable isotopes: ${ }^{58} \mathrm{Ni},{ }^{60} \mathrm{Ni},{ }^{61} \mathrm{Ni},{ }^{62} \mathrm{Ni}$ and ${ }^{64} \mathrm{Ni}$. It is the fifth most abundant element on Earth, although due to its siderophile behaviour, ca. 92\% resides in the core (McDonough, 2003). Despite its 


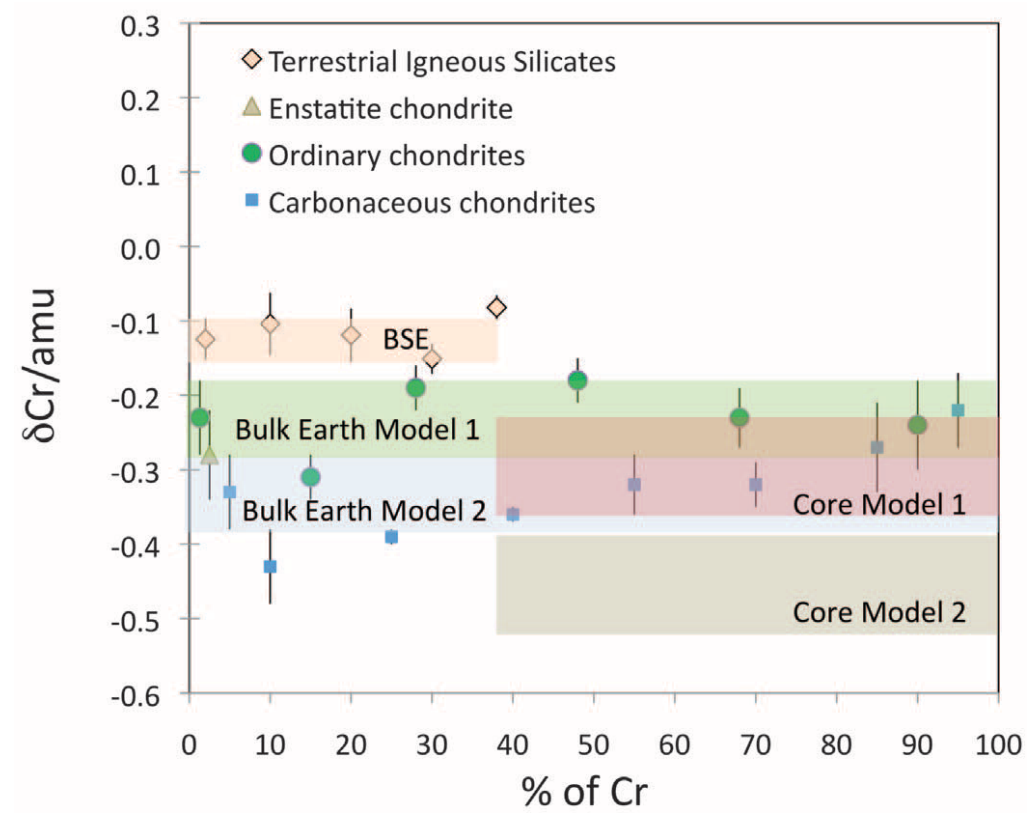

Figure 1.13: $\mathrm{Cr}$ isotopic compositions of terrestrial igneous silicates and chondritic meteorites, with modelled compositions for BSE (i.e., PUM), Earth's core, and the bulk Earth. Figure from Moynier et al. (2011)

enrichment in the core, $\mathrm{Ni}$ remains the ninth most abundant element in the silicate Earth (McDonough, 2003), and the seventh most abundant transition metal in the Earth's crust (Gall et al., 2012). Like all transition metals Ni can exist in a range of different oxidation states. However, unlike other transition metals it is essentially only present as $\mathrm{Ni}$ (II) in natural samples, and does not change state in redox reactions. Thus, any $\mathrm{Ni}$ stable isotope fractionations in nature must be caused by other processes, such as changes in speciation or coordination. Nickel also plays a key role in some biological reactions that are important in the carbon cycle (Ragsdale, 2007).

Most isotopic studies of $\mathrm{Ni}$ have focused on extraterrestrial materials, searching for nucleosynthetic anomalies or excesses in ${ }^{60} \mathrm{Ni}$, which may have been produced by decay of short-lived ${ }^{60} \mathrm{Fe}$ in the early Solar System, both of which can give insights into the astrophysical environment at the birth of the Solar System (Cook et al., 2006b; Quitté et al., 2006a b; Bizzarro et al. 
2007: Regelous et al., 2008; Cook et al., 2008; Chen et al., 2009, Steele et al., 2011).

Several studies have published Ni stable isotope measurements in terrestrial rocks (Cameron et al., 2009, Gall et al., 2012; Gueguen et al., 2013). These have shown that most terrestrial mantle and igneous rocks occupy a small range in Ni isotopic compositions compared with meteorites (Fig. 1.14). An obvious exception is found in komatiites and associated Ni-sulphide mineralisation, which show a large range of isotopic compositions that extend to much lighter compositions than are found in other materials. These have been attributed to high temperature magmatic processes relating to the production of sulphide mineralisation.

Mass-dependent $\mathrm{Ni}$ isotopic fractionations in meteoritic metals have been studied to investigate the formation processes and evolution of planetary cores. Metals from chondrites, pallasites and iron meteorites show a range of $\mathrm{Ni}$ isotopic compositions spanning ca. $0.5 \% 0 \mathrm{amu}^{-1}$ (Fig. 1.14) that follow a mass dependent fractionation trend (Cook et al., 2007; Moynier et al., 2007). Chondrites and iron meteorites have similar Ni isotopic compositions, which are distinct from that found in pallasite metals. The similarity between irons and chondrites is interpreted to reflect that they derived their Ni from a common stock. Isotopic variations in ordinary chondrites are attributed to reactions between solid and vapour phases and subsequent mineral sorting during accretion. Ni isotopic similarities are found between some groups of meteorites (e.g., IVA irons and L-chondrites, IIIAB and pallasites) that are consistent with hypothesised genetic links inferred from oxygen isotope data of Clayton and Mayeda (1996) (Moynier et al., 2007). The fact that $\mathrm{Ni}$ isotope data from all of the meteorite groups lie on a mass fractionation line from terrestrial samples implies that any nucleosynthetic anomalies in the solar nebula were homogenised by the time of planet accretion. No significant isotopic difference was found between Eagle Station and Main Group pallasites (Cook et al., 2007, Moynier et al., 2007). Cook et al. (2007) speculated that isotopic compositions of the pallasites may reflect fractionation 


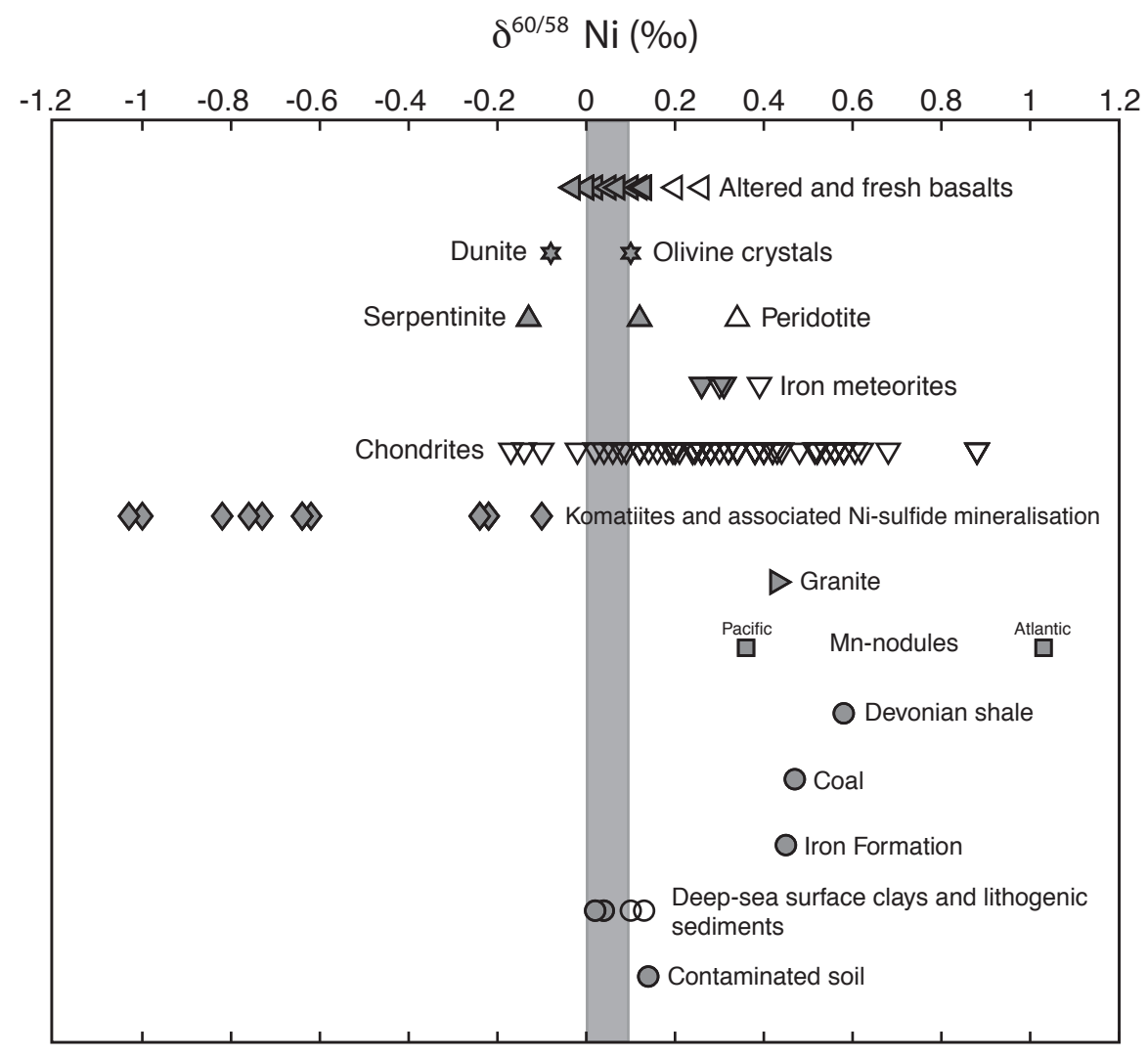

Figure 1.14: Ni stable isotopic compositions of terrestrial and extraterrestrial materials. Figure taken from (Gueguen et al. 2013), which includes literature data from Cook et al. (2007); Moynier et al. (2007); Cameron et al. (2009); Steele et al. (2011) represented by open circles. The grey bar represents the BSE (PUM) value as determined by Gueguen et al. (2013).

between metallic core and overlying mantle silicates, although recent measurements of equilibrium $\mathrm{Ni}$ isotope fractionation between metal and silicate phases (Lazar et al., 2012) suggest that metal-silicate differentiation processes are unlikely to result in significant $\mathrm{Ni}$ isotopic fractionation.

IIIAB irons are considered to sample a wide range of degrees of fractional crystallisation of the metallic core of an asteroidal parent body (e.g. Haack and Scott, 1993). Ni isotope compositions in IIIAB irons were found to span the entire range of compositions found in magmatic irons (Cook et al., 2006a). However, the isotopic variations do not vary between the two putative end 
members of the core crystallisation sequence (early crystallising IIIA, and late crystallising IIIB) and do not correlate with Ni content, suggesting that core crystallisation did not significantly fractionate Ni isotopes Cook et al. 2006a). Thus, Ni isotopic variations in iron meteorites are instead considered to reflect a combination of events including fractionation between 1) liquid metal and silicate during core segregation, 2) immiscible melts in a molten core, and 3) metallic and non-metallic (i.e., sulphide or phosphide) phases during core solidification (Cook et al., 2006a, 2007).

The relative proportions and size distribution of different $\mathrm{Fe}-\mathrm{Ni}$ alloy phases (kamacite and taenite) in iron meteorites have been used to study cooling rates in asteroidal cores (e.g., Wood, 1964, Goldstein and Ogilvie, 1965, Haack and Scott, 1993; Hopfe and Goldstein, 2001; Yang and Goldstein, 2006), which are important in constraining the size of these planetary bodies and the heat source responsible for their differentiation. Clearly resolvable (ca. $0.36 \% 0 \mathrm{amu}^{-1}$ ) Ni stable isotopic fractionations between these phases in iron meteorites have been reported (Bourdon et al., 2006, Quitté et al. 2006a; Cook et al., 2007). The Ni isotopic fractionation between the alloy phases is thought to be a kinetic effect related to diffusion of $\mathrm{Ni}$ during cooling and development of the Widmanstätten pattern (Cook et al., 2007. Dauphas, 2007). Ni isotopic measurements combined with computer models can place tighter constraints on cooling rates in the cores of asteroidal parent bodies (Dauphas, 2007). Fe and Ni stable isotope variations in iron meteorites do not show any correlation (Cook et al., 2007), suggesting that whatever process fractionated Ni isotopes did not affect Fe isotopes to same degree.

\subsubsection{Platinum stable isotopes}

Platinum has six naturally occurring isotopes $-{ }^{190} \mathrm{Pt},{ }^{192} \mathrm{Pt},{ }^{194} \mathrm{Pt},{ }^{195} \mathrm{Pt}$, ${ }^{196} \mathrm{Pt}$ and ${ }^{198} \mathrm{Pt}$ - with relative abundances of ca. $0.01 \%, 0.78 \%, 32.86 \%$, $33.78 \%, 25.21 \%$ and $7.36 \%$, respectively (Berglund and Wieser, 2011). Only ${ }^{190} \mathrm{Pt}$ is radioactive $\left(\mathrm{t}_{1 / 2} \sim 10^{11}\right.$ years $)$ and despite its long half-life, is the 
basis of the ${ }^{190} \mathrm{Pt}-{ }^{186} \mathrm{Os}$ decay system that has been used to infer interactions between Earth's core and mantle (Walker et al., 1995, Brandon et al., 1998; Brandon and Walker, 2005). Pt is a transition metal with a range of possible oxidation states, but principally occurs as $\mathrm{Pt}^{0}, \mathrm{Pt}^{2+}$ and $\mathrm{Pt}^{4+}$ Cotton and Wilkinson, 1966). Pt is highly chemically unreactive and refractory. It is also highly siderophile, and is strongly partitioned into $\mathrm{Fe}-\mathrm{Ni}$ cores of terrestrial planets during planetary differentiation. Compared with some other nontraditional stable isotope systems, including those discussed previously in this section, the geochemistry of Pt is relatively simple: Pt is refractory, it is highly-siderophile and thus ultra-depleted in Earth's mantle, and it is not biologically utilised.

\section{Pt stable isotope testing of accretion and differentiation models for Earth}

As described in Section 1.3, two main competing models for Earth's accretion and differentiation are currently favoured in the literature: 1) homogeneous accretion with core formation occurring at the base of a deep $(\geq 700 \mathrm{~km})$ magma ocean, and 2) heterogeneous accretion with core formation at lower temperatures and pressures followed by accretion of a late-veneer of oxidised chondritic material after formation of the core that was subsequently mixed into the upper mantle. While both of these scenarios have wide support and compelling supporting evidence, at present neither of these models can satisfactorily explain all of the available constraints. Alternative methods for investigation of these problems are desirable, and non-traditional stable isotope systems, such as those described in this section, have begun to play a role in this debate. For example, Fe isotope compositions of Earth and the Moon have been shown to be consistent with fractionation by disproportionation in a magma ocean (Gessmann et al., 2001; Williams et al., 2006. Shahar et al., 2009), although this model has further implications such as a greater than Mars-sized Moon-forming impactor or that the fraction of the Lunar mantle that is derived from the impactor is less than previously sug- 
gested (Williams et al., 2006). Similarly, stable .isotopic compositions of Cr, which is thought to be partitioned into the core during core formation ( $\mathrm{Mc}$ Donough, 2003, Ringwood et al., 1990; Allègre et al., 1995), show distinct differences between the mantle and chondrites, which has been suggested to reflect fractionation in a magma ocean at mid-mantle depth (Moynier et al. 2011). The addition of a highly siderophile stable isotope system, like Pt, may provide new insights into these processes. The different hypotheses for accretion and core formation are likely to have fractionated $\mathrm{Pt}$ stable isotopes differently, resulting is distinct isotopic fingerprints in Earth's mantle. By measuring the composition of Earth's mantle and comparing that with compositions of different types of meteorites, this study aims to constrain the main source of siderophile elements in Earth's mantle.

Given the extreme leverage of metals over siderophile elements, extraction of siderophile elements into the core is expected to result in relatively large $\mathrm{Pt}$ stable isotopic fractionations in the complementary mantle, particularly if changes in oxidation state were involved. Terrestrial mantle samples, i.e., xenolith and massif peridotites, were measured to constrain the composition of PUM. As described in Section 1.3, the Earth is considered to have a bulk composition similar to chondrites. Thus, the Pt stable isotopic composition of chondrite meteorites yields the composition of the undifferentiated Earth, which provides a starting composition against which any isotopic fractionation can be referenced. A large offset between the chondritic and mantle compositions would be indicative of an isotopic fingerprint of core formation. Chondrites are also thought to represent the composition of the late-veneer material, and thus a chondrite composition in the mantle would represent a fingerprint of the late-veneer. Other meteorite groups used in this study also provide samples of planetesimals at different stages of planetary differentiation, providing a means to investigate the change in isotopic compositions through these processes, and place minimum constraints on the direction and magnitude of isotopic fractionations resulting from metal-silicate planetary differentiation. 
Table 1.3: Thermal neutron capture cross sections of nuclides in the mass range of $\mathrm{Pt}$.

\begin{tabular}{lccccccccc}
\hline Isotope & ${ }^{190} \mathrm{Pt}$ & ${ }^{191} \mathrm{Ir}$ & ${ }^{192} \mathrm{Pt}$ & ${ }^{193} \mathrm{Ir}$ & ${ }^{194} \mathrm{Pt}$ & ${ }^{195} \mathrm{Pt}$ & ${ }^{196} \mathrm{Pt}$ & ${ }^{197} \mathrm{Au}$ & ${ }^{198} \mathrm{Pt}$ \\
Relative abundance $\left.{ }^{\mathrm{a}} \%\right)$ & 0.01 & 37.3 & 0.78 & 62.7 & 33 & 33.8 & 25.2 & 100 & 7.16 \\
Cross section $^{\mathrm{b}}$ (barns) & 147 & 954 & 10 & 111 & $\sim 1$ & 28 & $\sim 0.6$ & 99 & 3.7 \\
\hline
\end{tabular}

${ }^{\mathrm{a}}$ Isotope abundances from Berglund and Wieser (2011); ${ }^{\mathrm{b}}$ Thermal neutron capture cross sections from Mughabghab (2003).

\section{Considerations for $\mathrm{Pt}$ stable isotope studies of meteorites}

Recently, Pt isotope ratios in meteorite have been proposed for use as a dosimeter for cosmogenic effects in iron meteorites that can disturb the Hf-W isotope system (Kruijer et al., 2013; Wittig et al., 2013). The utility of Pt for this purpose derives from the fact that ${ }^{191} \mathrm{Ir}$, which comprises $37.3 \%$ of Ir (Table 1.3), has a large thermal neutron capture cross section and is converted through neutron capture to ${ }^{192} \mathrm{Pt}$, which has a low natural abundance of $0.78 \%$ (Table 1.3 . Thus, large ${ }^{192} \mathrm{Pt}$ anomalies can be produced during exposure of meteorites to cosmic radiation. Based on the isotopic abundances and thermal neutron capture cross-sections shown in Table 1.3, there is potential for mass-independent isotopic effects on several Pt stable isotope ratios, which could significantly disturb Pt stable isotope measurement, particularly in iron meteorites.

These effects must be taken into account when applying this isotope system to meteorite samples, particularly when using a double-spike without an unspiked sample measurement, which assumes that all fractionation is massdependent, and care must be taken in the interpretation of data. Cosmogenic effects would be expected to have the most severe impact on iron meteorites with long cosmic ray exposure (CRE), and cosmogenic effects may be evident through correlations between $\mathrm{Pt}$ stable isotope ratios and CRE ages. There may also be potential to apply a cosmogenic correction to Pt stable isotopes using another isotope system (e.g., Os), similar to the use of Pt isotope ratios for correcting W isotope data (Kruijer et al., 2013, Wittig et al., 2013). 


\subsection{Thesis structure}

This research largely comprised the development of experimental methods, which is reflected in the structure of this thesis. Two chapters are dedicated to describing the major technical developments - protocols for chemical separation of Pt from natural samples, and methods for isotopic measurement by mass spectrometry. The later part of this thesis is concerned with the application of these techniques to tracing the accretion and differentiation of Earth through measurement of terrestrial and meteoritic samples. The chapters of this thesis are structured around these key objectives.

Chapter 1 introduces the $\mathrm{Pt}$ stable isotope system in the context of its geochemical behaviour and of other non-traditional stable isotope systems that have been applied to study similar high temperature planetary processes. It also includes a broad overview of terrestrial planet formation and outlines the unresolved problems to which the analytical techniques will be applied in this thesis.

The chemical and mass spectrometric methods developed in this work are presented separately in Chapters 2 and 3. Chapter 2 presents the method for $\mathrm{Pt}$ stable isotope ratio measurements by double-spike MC-ICPMS. Chapter 3 presents the protocols for chemical separation of $\mathrm{Pt}$ from natural samples, and includes Pt stable isotope data for a number of internationally available terrestrial standard reference materials.

Chapter 4 presents results of the application of these techniques to terrestrial and meteorite samples to investigate outstanding problems associated with the formation and differentiation of Earth.

Chapter 5 presents the conclusions of this thesis, with suggestions for future work.

Three appendices are also attached to this thesis. Appendix A contains detailed information on the terrestrial and meteorite samples used in this study, including photographs and reference data. Appendix B contains a reprint of the published version of the manuscript presented in Chapter 2 . 
Lastly, a new computer tool was developed during this research for processing of double-spike mass spectrometry data. This tool, called IsoSpike, has been described in a manuscript that, at the time of writing, is currently under review for publication in Geostandards and Geoanalytical Research. A draft version of this manuscript is included as Appendix C. 


\section{Chapter 2}

\section{Mass spectrometry}

This chapter was prepared as a journal article and is now published in the Journal of Analytical Atomic Spectrometry* ${ }^{*}$. The article is entitled Platinum Stable Isotope Ratio Measurements by Double-Spike Multiple Collector $I C P M S$. References have been gathered to the combined references section at the end of this thesis. The published article is included with this thesis as Appendix B. This chapter also describes reduction of double-spike data using a tool put forward by Creech and Paul (2014). This tool is now named IsoSpike, and a preprint of the paper describing, currently in press in Geostandards and Geoanalytical Research, is included as Appendix D.

प्य

\section{Abstract}

We present a new technique for the precise determination of platinum (Pt) stable isotope ratios by multiple-collector inductively coupled plasma mass spectrometry (MC-ICPMS) using two different Pt double-spikes $\left({ }^{192} \mathrm{Pt}-{ }^{198} \mathrm{Pt}\right.$ and $\left.{ }^{196} \mathrm{Pt}-{ }^{198} \mathrm{Pt}\right)$. Results are expressed relative to the IRMM-010 Pt isotope

* Author list: John Creech, Joel Baker, Monica Handler, Martin Schiller and Martin Bizzarro

$\dagger$ DOI: $10.1039 / \mathrm{c} 3 \mathrm{ja} 50022 \mathrm{e} /$ 
standard as the parts per million difference in ${ }^{198} \mathrm{Pt} /{ }^{194} \mathrm{Pt}$ ratios $\left(\mu^{198} \mathrm{Pt}\right)$. Repeated measurements of the IRMM-010 Pt standard in two different laboratories, consuming ca. 40-85 ng of $\mathrm{Pt}$, show that a long-term external reproducibility for $\mu^{198} \mathrm{Pt}$ of $\leq 40 \mathrm{ppm}\left(2 \mathrm{sd}\right.$; equivalent to $\leq 10 \mathrm{ppm} \mathrm{u}^{-1}$, where $\mathrm{u}$ is the unified atomic mass unit) can be obtained on Pt stable isotope ratios with either double-spike. Elemental doping tests reveal that double-spike corrected $\mathrm{Pt}$ stable isotope ratios are insensitive to the presence of relatively high (up to $10 \%$ ) levels of matrix elements, although the ${ }^{192} \mathrm{Pt}-{ }^{198} \mathrm{Pt}$ double-spike is affected by an isobaric interference on ${ }^{192} \mathrm{Pt}$ from ${ }^{192} \mathrm{Os}$. The ${ }^{196} \mathrm{Pt}-{ }^{198} \mathrm{Pt}$ double-spike does not use ${ }^{192} \mathrm{Pt}$ in the double-spike inversion and is unaffected by Os contamination, and is our recommended double-spike for use with natural samples. As part of this study, we re-determined the natural Pt isotopic composition of IRMM-010 by MC-ICPMS using external element $(\mathrm{Pb})$ doping to correct for instrumental mass bias and have identified relative $\mathrm{Pt}$ isotope differences of up to $10 \%$ from the reference values for this standard. The new isotopic composition of the IRMM-010 standard $\left({ }^{190} \mathrm{Pt}=0.01289 \%,{ }^{192} \mathrm{Pt}=0.7938 \%,{ }^{194} \mathrm{Pt}=32.81 \%,{ }^{195} \mathrm{Pt}=33.79 \%,{ }^{196} \mathrm{Pt}\right.$ $=25.29 \%$ and ${ }^{198} \mathrm{Pt}=7.308 \%$ ) results in a redefined $\mathrm{Pt}$ atomic weight of 195.08395 \pm 0.00068 . Using our technique we have measured small, reproducible and statistically significant offsets in $\mathrm{Pt}$ stable isotope ratios between different Pt element standards and the IRMM-010 standard, which potentially indicates that natural Pt stable isotope fractionations exist that are larger than the reproducibility of our technique.

\section{$2.1 \quad$ Introduction}

A wide range of novel, non-traditional, stable isotope systems have recently been developed, largely as a result of the advent of multiple-collector inductively coupled plasma mass spectrometry (MC-ICPMS). These stable isotope systems are providing valuable new insights in the earth, environmental and planetary sciences (e.g. Halliday et al., 1998; Albarède, 2004; Anbar, 2004 
Beard and Johnson, 2004, Young and Galy, 2004, Anbar and Rouxel, 2007, Bizzarro et al., 2011). The platinum (Pt) stable isotope system represents a potentially powerful, but as yet unexplored, addition to this new suite of stable isotope systems. Platinum is a highly siderophile transition metal with six naturally occurring isotopes - ${ }^{190} \mathrm{Pt},{ }^{192} \mathrm{Pt},{ }^{194} \mathrm{Pt},{ }^{195} \mathrm{Pt},{ }^{196} \mathrm{Pt}$ and ${ }^{198} \mathrm{Pt}$ - with relative abundances of $\sim 0.01 \%, 0.78 \%, 32.86 \%, 33.78 \%, 25.21 \%$ and $7.36 \%$, respectively (Berglund and Wieser, 2011). Only ${ }^{190} \mathrm{Pt}$ is radioactive $\left(\mathrm{t}_{1 / 2} \sim 10^{11} \mathrm{yr}\right)$, and despite its long half-life, is the basis of the ${ }^{190} \mathrm{Pt}-{ }^{186} \mathrm{Os}$ decay system that has been used to infer interactions between Earth's core and mantle (Brandon and Walker, 2005). Platinum can exist in a range of oxidation states, but principally occurs as $\mathrm{Pt}^{0}, \mathrm{Pt}^{2+}$ and $\mathrm{Pt}^{4+}$ Cotton and Wilkinson, 1966). Unlike most previously studied non-traditional stable isotope systems, Pt is not utilised by any known essential biological mechanisms. However, the relatively large mass difference $(2 \%)$ between the abundant heavy and light isotopes of $\mathrm{Pt}$, coupled with its variable redox states in the Earth's core, mantle and surficial environment, means that it has potential to exhibit significant stable isotope variations in Earth materials. The bulk of Earth's Pt budget is thought to reside in the core as evidenced by the relative depletion of $\mathrm{Pt}$ and other highly siderophile elements in Earth's upper mantle relative to chondritic meteorites (e.g. Righter, 2003). Typical Pt concentrations in mantle rocks are ca. 1-100 ng $\mathrm{g}^{-1}$ as compared with 1-10 $\mu \mathrm{g} \mathrm{g}^{-1}$ in chondrites, iron meteorites and, presumably, Earth's core. As a result, the relative distributions of elements such as Pt between these different reservoirs are important in constraining models of planetary differentiation (Righter, 2003; Lorand et al., 2008). Enrichments in Pt relative to the other platinum group elements (PGE; Rh, Ru, Pd, Os and Ir) have also been observed in authigenic marine sediments such as ferromanganese crusts and nodules (Hodge et al., 1985, Colodner, 1991; Halbach et al., 1989. Goldberg and Koide, 1990). This Pt enrichment may be related to its variable oxidation states (Hodge et al., 1985; Banakar et al., 2007). As such, Pt stable isotopes hold considerable promise for use as an isotopic tracer 
for redox in surficial and marine environments and for processes relating to planetary accretion and differentiation.

In addition to being a potentially valuable geochemical tracer, $\mathrm{Pt}$ has a wide range of uses, including industrial, medical and scientific, where other potential applications for a Pt stable isotope tracer may be found. Various $\mathrm{Pt}$ compounds are used in medicine and, in particular, several different $\mathrm{Pt}$ complexes (cisplatin, carboplatin and oxaliplatin) are used in chemotherapy drugs (Roberts et al., 1979; Abrams and Murrer, 1993; Guo et al., 1999; Ho et al. 2003). Platinum has applications in various manufacturing processes, usually as a catalyst, and is widely used in the automotive industry in catalytic converters (Heck and Farrauto, 1995; Kašpar et al., 2003). Given its use in automotive catalytic converters, $\mathrm{Pt}$ is also used for environmental monitoring of vehicle emissions by measuring $\mathrm{Pt}$ concentrations in roadside soils (Ek et al. 2004, Ely et al. 2001).

Despite the potential range of uses for Pt stable isotopes, previous isotopic studies of Pt have been extremely limited. White et al. (1956) measured the isotopic composition of $\mathrm{Pt}$ using a double focusing mass spectrometer with a thermal ionisation source (Table 2.1). This Pt isotopic composition was used in IUPAC reports of elemental isotopic compositions and atomic weights until 1995, when a revised isotopic composition determined by Taylor et al. (1994) using gas source mass spectrometry and electron impact ionisation of gaseous $\mathrm{Pt}\left(\mathrm{PF}_{3}\right)_{4}$ was adopted (Coplen, 1997, Taylor et al., 1994)(Table 2.1. In the late 1990s, two studies documented procedures for measurement of Pt isotopes by negative thermal ionisation mass spectrometry (N-TIMS) (Briche et al. 1997; Hattori et al., 1998). One of the groups subsequently developed a Pt isotope reference material (IRMM-010) for the Institute for Reference Materials and Measurements, for which certified values were determined using quadrupole ICPMS (Wolff Briche et al., 2002) (Table 2.1). Later studies of IRMM-010 have been limited to its use in testing the analytical performance of axial inductively coupled plasma time-of-flight mass spectrometer instruments (Emteborg et al., 2000, Tian et al., 1999). Two 
recently published studies used internally normalised Pt isotope ratios as a dosimeter for neutron capture in iron meteorites (Kruijer et al., 2013, Wittig et al. 2013). However, to-date there has been no investigation of the Pt stable isotopic system.

Herein, we describe a method for highly precise determination of Pt stable isotope ratios using ${ }^{192} \mathrm{Pt}-{ }^{198} \mathrm{Pt}$ and ${ }^{196} \mathrm{Pt}-{ }^{198} \mathrm{Pt}$ double-spikes (DS) to correct for instrumental mass bias. We show that this approach is capable of measuring Pt stable isotopes on ca. 40-85 ng of natural Pt with an external reproducibility of $\leq \pm 10 \mathrm{ppm} \mathrm{u}^{-1}(2 \mathrm{sd}$; $\mathrm{u}$ is the unified atomic mass unit), and identify measurable Pt stable isotope variations amongst commercially available Pt standards. Moreover, during the course of this work, we identified that the previously adopted abundances of some Pt isotopes are in relative terms up to $10 \%$ inaccurate and, as such, we present a revised schedule of the isotopic abundances and atomic weight of Pt.

\subsection{Double-spike methodology}

The DS technique has been extensively used in highly precise $\mathrm{Pb}$ isotope analysis, and has also been applied to a wide range of other isotope systems, including S, Ca, Cr, Zn, Fe, Mo, Cd, Ba and Os, by both MC-ICPMS and TIMS analysis (e.g. Anbar et al., 2001; Arnold et al., 2010; Baker et al. 2004; Beard and Johnson, 1999; Beard et al., 1999; Dideriksen et al., 2006; Eugster et al., 1969; Gopalan et al., 2006; Mann and Kelly, 2005; Markey et al., 2003; Ripperger and Rehkämper, 2007; Rosman, 1972; Schoenberg et al., 2008, Siebert et al., 2001). The fundamentals of the DS approach for isotopic analysis have been well documented (Dodson, 1963, Compston and Oversby, 1969; Hamelin et al., 1985; Powell et al., 1998; Galer, 1999; Johnson and Beard, 1999, Rudge et al., 2009), and mathematical solutions have been developed utilising both algebraic (Rudge et al., 2009; Albarède and Beard, 2004) and geometric (Siebert et al., 2001; Galer, 1999; Johnson and Beard, 1999, Russell, 1971, Markey et al., 2003) approaches. Here we 


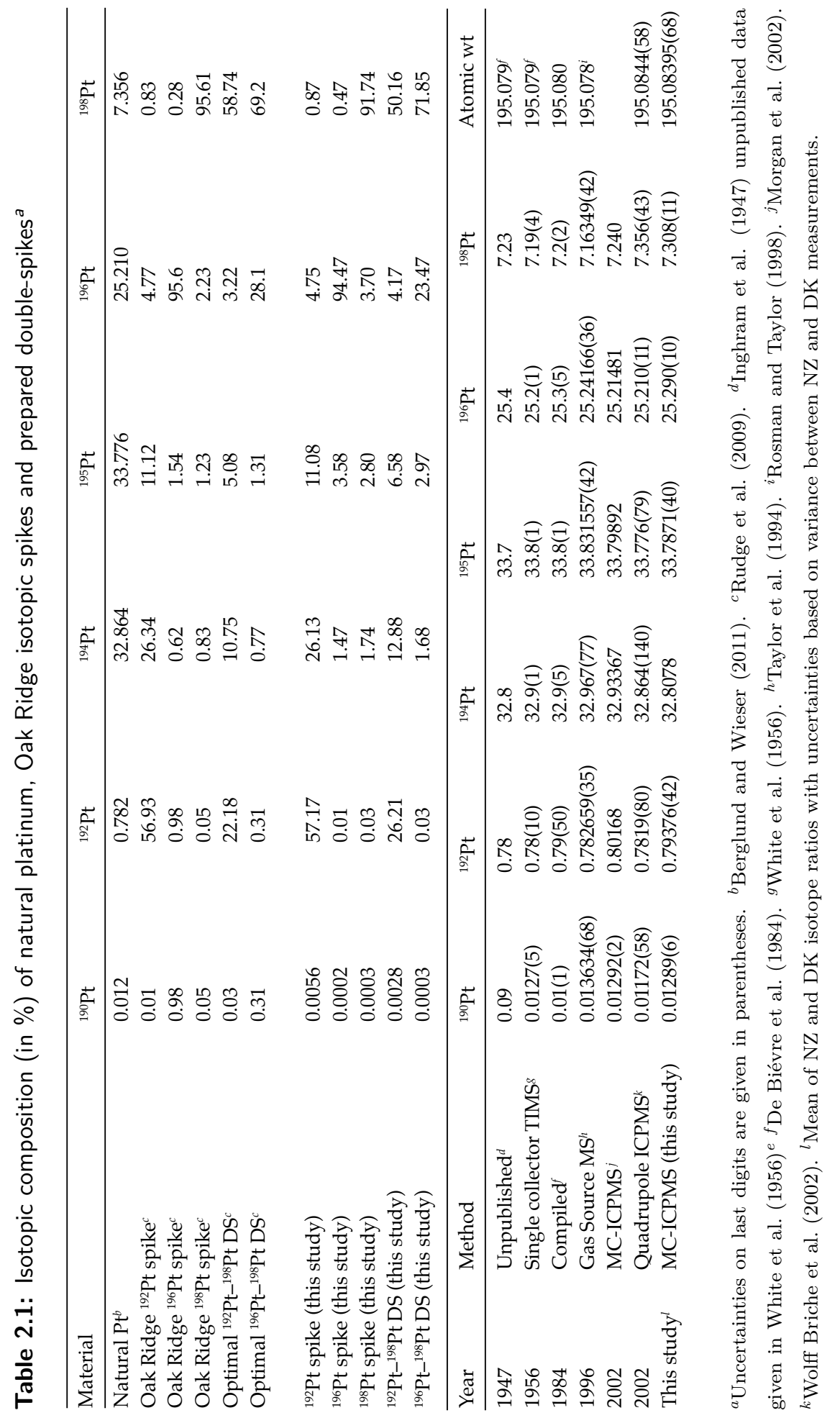


use the algebraic method of Rudge et al. (2009) (Rudge et al. 2009).

The DS method is commonly accepted as the most rigorous method available for correcting for instrumental mass fractionation as it has several advantages over other methods such as standard-sample bracketing, which are: (1) for stable isotope systems where mass-dependent fractionation is being studied, DS-corrected isotope ratios can be obtained from a single analysis (Rudge et al., 2009); (2) the DS can potentially correct for any mass-dependent effects to the sample after addition of the DS, such as isotopic fractionations that might occur as a result of incomplete yields during chemical separation of the element from natural samples (Rudge et al. 2009 Albarède and Beard, 2004); (3) the DS method has been shown to be less sensitive to the presence of minor amounts of remaining matrix elements than sample-standard bracketing methods (Dideriksen et al., 2006).

\subsubsection{Pt double-spike design}

With five stable isotopes and one with an extremely long half-life $\left({ }^{190} \mathrm{Pt}\right.$, $\mathrm{t}_{1 / 2} \sim 10^{11} \mathrm{yr}$ ), Pt is ideally suited to the DS approach. Error propagation in DS calculations needs to be minimised by selection of an optimal spike composition and sample-spike mixtures. A database of theoretically ideal spike compositions and spike/sample ratios for all elements with four or more naturally occurring isotopes was presented by Rudge et al. (2009), which includes optimal DS compositions based on available spikes from the Oak Ridge National Laboratory, USA. Two platinum DS were prepared as part of this study, as described below.

\section{${ }^{192} \mathrm{Pt}-{ }^{198} \mathrm{Pt}$ double-spike}

The ${ }^{192} \mathrm{Pt}-{ }^{198} \mathrm{Pt}$ DS comprises a mixture of $38.9 \%{ }^{192} \mathrm{Pt}$ and $61.1 \%{ }^{198} \mathrm{Pt}$ spikes. This DS combination has one of the smallest error propagations predicted by the double-spike toolbox of Rudge et al. (2009) (ca. $32 \mathrm{ppm} \mathrm{u}^{-1}$, 
$1 \mathrm{sd})$. The ${ }^{192} \mathrm{Pt}-{ }^{198} \mathrm{Pt}$ DS inversion was performed using ${ }^{192} \mathrm{Pt},{ }^{194} \mathrm{Pt},{ }^{196} \mathrm{Pt}$ and ${ }^{198} \mathrm{Pt}$. Given that the spiked isotopes ${ }^{192} \mathrm{Pt}$ and ${ }^{198} \mathrm{Pt}$ have relatively low natural abundances $(0.78 \%$ and $7.36 \%$, respectively; Berglund and Wieser 2011) and the two unspiked isotopes in the inversion $\left({ }^{194} \mathrm{Pt}\right.$ and $\left.{ }^{196} \mathrm{Pt}\right)$ have high natural abundances, this DS permits precise isotopic determinations over a wide range of sample/spike ratios. The use of this DS is, however, complicated by a potentially large direct isobaric interference from Os on ${ }^{192} \mathrm{Pt}$ and the fact that, in some natural materials that might be targets for $\mathrm{Pt}$ stable isotope analysis, $\mathrm{Pt}$ and $\mathrm{Os}$ are both of similar abundance (e.g. chondritic and iron meteorites, Earth's mantle). ${ }^{190} \mathrm{Pt}$ and ${ }^{192} \mathrm{Pt}$ isotopes have isobars with abundant isotopes of osmium $\left({ }^{190} \mathrm{Os}\right.$ and ${ }^{192} \mathrm{Os}$ with relative abundances of $26.2 \%$ and $40.8 \%$, respectively). Thus, the presence of even small amounts of Os in a sample can significantly affect the Pt signal on those masses, producing inaccurate ${ }^{192} \mathrm{Pt}-{ }^{198} \mathrm{Pt}$ DS corrected ratios.

${ }^{196} \mathrm{Pt}-{ }^{198} \mathrm{Pt}$ double-spike

The ${ }^{196} \mathrm{Pt}-{ }^{198} \mathrm{Pt}$ DS comprises a mixture of $27.7 \%{ }^{196} \mathrm{Pt}$ and $72.3 \%{ }^{198} \mathrm{Pt}$ spikes. The hypothetical error propagation on the ${ }^{196} \mathrm{Pt}-{ }^{198} \mathrm{Pt}$ DS is slightly larger than that of the ${ }^{192} \mathrm{Pt}-{ }^{198} \mathrm{Pt}$ DS (ca. $40 \mathrm{ppm} \mathrm{u}^{-1} ; 1 \mathrm{sd}$ ). However, as alluded to above, the ${ }^{196} \mathrm{Pt}-{ }^{198} \mathrm{Pt}$ DS has some potential advantages over the ${ }^{192} \mathrm{Pt}-{ }^{198} \mathrm{Pt}$ DS in that the ${ }^{196} \mathrm{Pt}-{ }^{198} \mathrm{Pt}$ double-spike inversion is performed on masses ${ }^{194} \mathrm{Pt},{ }^{195} \mathrm{Pt},{ }^{196} \mathrm{Pt}$ and ${ }^{198} \mathrm{Pt}$, and this DS should thus be unaffected by the presence of Os.

\subsection{Materials and methods}

\subsubsection{Chemicals and standard solutions}

Optima ${ }^{\mathrm{TM}}$ grade nitric $\left(\mathrm{HNO}_{3}\right)$ and hydrochloric $(\mathrm{HCl})$ acids were used as supplied without further purification. Acid dilutions were performed with 
ultra-pure (>18 M $\Omega . c m)$ water. All sample handling and preparation was performed in a class-100 ultra-clean laboratory at Victoria University of Wellington.

The Pt isotope standard IRMM-010 was purchased from the Institute for Reference Materials and Measurements, Belgium (Wolff Briche et al. 2002). The standard was supplied in the form of Pt wire segments, which were cleaned prior to digestion by sequentially rinsing in ultra-pure water, analytical grade methanol and dilute $(5 \%) \mathrm{HNO}_{3}$. The IRMM-010 material was carefully weighed, and then digested in aqua regia $\left(\mathrm{HCl}: \mathrm{HNO}_{3}\right.$ prepared in a volume ratio of 3:1) in a Savillex ${ }^{\mathrm{TM}}$ teflon beaker. The IRMM-010 Pt solution was dried down, refluxed with concentrated $\mathrm{HNO}_{3}$, evaporated to dryness, and finally redissolved in $7 \mathrm{M} \mathrm{HNO}_{3}$. At the time this standard was prepared, sample introduction was carried out in $\mathrm{HNO}_{3}$. This was later changed to $\mathrm{HCl}$, therefore a portion of this standard was converted to chloride form and stored in $20 \% \mathrm{HCl}$.

Two commercially available Pt ICPMS standards were used in this study: J.T. Baker $1000 \mu \mathrm{g} \mathrm{mL} \mathrm{m}^{-1} \mathrm{Pt}$ ICP std in 20\% HCl (Lot\# H44N77), and SPEX CertiPrep $1000 \mu \mathrm{gL}^{-1} \mathrm{Pt}$ ICP standard in 10\% $\mathrm{HCl}$ from Fisher Scientific (Lot\# 15-181PT; hereafter referred to as the Baker and Spex standards, respectively). Additionally, a piece of Pt metal ribbon used for TIMS filaments was obtained and digested for use as an in-house standard (hereafter referred to as the Ribbon standard). The Pt Ribbon standard was pre-cleaned and digested in the same manner as described above for IRMM-010. The digested filament was evaporated to dryness several times in concentrated $\mathrm{HCl}$, and finally redissolved in $20 \% \mathrm{HCl}$.

\subsubsection{Preparation of platinum double-spikes}

Three single Pt isotope spikes $\left({ }^{192} \mathrm{Pt},{ }^{196} \mathrm{Pt}\right.$ and $\left.{ }^{198} \mathrm{Pt}\right)$ were obtained from the Oak Ridge National Laboratory, USA (Batch numbers 186140, 235601 and 235701, respectively). Reference Pt isotopic compositions for each isotopic spike are shown in Table 2.1 as well as the measured compositions obtained 
in this study. The ${ }^{192} \mathrm{Pt}$ spike was supplied as a segment of Pt metal ribbon, and the ${ }^{196} \mathrm{Pt}$ and ${ }^{198} \mathrm{Pt}$ spikes were provided as very fine-grained metallic powders. The ${ }^{192} \mathrm{Pt}$ spike ribbon was sequentially cleaned prior to digestion by rinsing with ultra-pure water, analytical grade methanol and dilute (5\%) $\mathrm{HNO}_{3}$. Each spike was digested independently with aqua regia in Savillex ${ }^{\mathrm{TM}}$ teflon beakers. Once the spike material had been digested, the spike solutions were repeatedly evaporated to dryness in concentrated $\mathrm{HCl}$, and finally redissolved in $20 \% \mathrm{HCl}$.

Aliquots of each single spike solution were taken to determine their concentrations (and isotopic compositions). These were measured by MCICPMS using a standard bracketing approach with the IRMM-010 standard. The two DS were then prepared by mixing the appropriate two spikes in the proportions calculated for optimal ${ }^{192} \mathrm{Pt}-{ }^{198} \mathrm{Pt}$ and ${ }^{196} \mathrm{Pt}-{ }^{198} \mathrm{Pt}$ DS mixtures as given by Rudge et al. (Rudge et al., 2009). Each DS mixture was then evaporated to dryness, taken up in a small volume of concentrated $\mathrm{HCl}$, evaporated to dryness again, and finally taken up in $6 \mathrm{M} \mathrm{HCl}$. Accurate isotopic determination by the DS technique requires calibration of the DS composition. The method for calibration of the DS prepared here and the accuracy of the calibrations are described below.

Platinum standards were spiked immediately prior to MC-ICPMS measurement in proportions close to that for optimal spiking as given in Rudge et al. (2009). Reference Pt isotopic compositions for each isotopic spike and DS are shown in Table 2.1 along with the compositions measured by MC-ICPMS for the spikes and DS used in this study.

\subsubsection{Mass spectrometry}

Platinum isotope measurements were performed using MC-ICPMS instruments in two different laboratories. Most analyses were carried out with a $\mathrm{Nu}$ Plasma HR MC-ICPMS (Nu Instruments) at Victoria University of Wellington, New Zealand. Repeated measurements of selected standards were also undertaken with a ThermoFisher Neptune MC-ICPMS located at the 
Table 2.2: Instrumental operating and measurement conditions

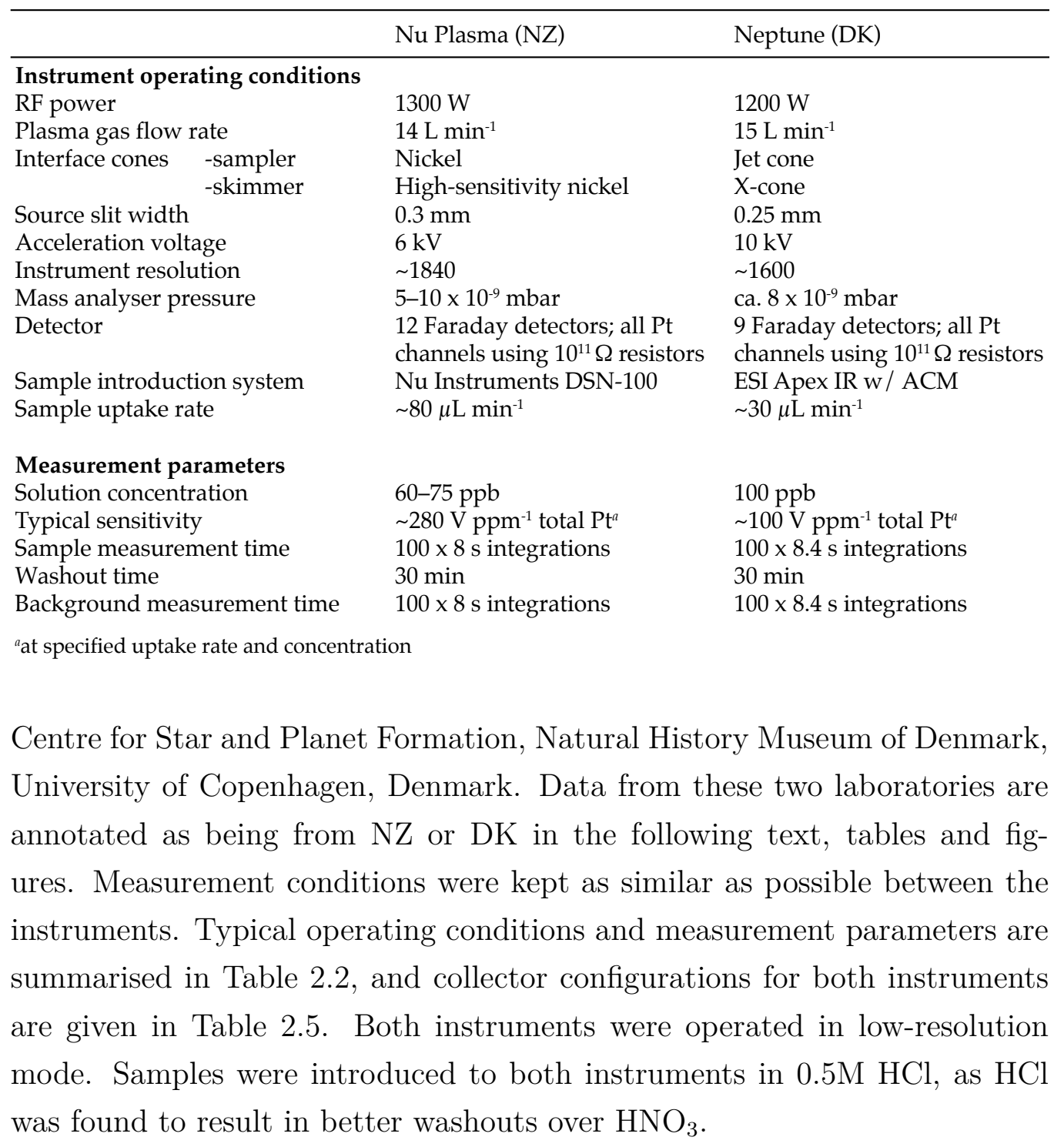

\section{Nu Plasma MC-ICPMS (NZ)}

The Nu Plasma MC-ICPMS was operated in low-resolution mode, with a mass resolution of $\sim 1840$ (M/ $\Delta \mathrm{M}$; peak edge width from $5-95 \%$ full peak height). A Nu Instruments DSN-100 desolvation nebuliser system with an 
ESI PFA micro-flow nebuliser was used for Pt solution introduction to the mass spectrometer. Measurements were typically made on $0.5 \mathrm{M} \mathrm{HCl}$ solutions with 75 or $60 \mathrm{ng} \mathrm{mL}-1$ (for the ${ }^{192} \mathrm{Pt}-{ }^{198} \mathrm{Pt}$ or ${ }^{196} \mathrm{Pt}-{ }^{198} \mathrm{Pt} \mathrm{DS}$, respectively) of natural $\mathrm{Pt}$, with an almost equivalent contribution from DS Pt. The sample uptake rate was ca. $80 \mu \mathrm{L} \mathrm{min}^{-1}$, and the sensitivity of the instrument was typically ca. $280 \mathrm{~V} \mathrm{ppm}^{-1}$ total $\mathrm{Pt}$ under these conditions. Peak centering was performed at the start of each session and checked at the end of the session. Each individual analysis comprised $100 \times 8$ s integrations, corresponding to $13.3 \mathrm{~min}$ of data acquisition, and consumed ca. $80 \mathrm{ng}$ of natural Pt. Baselines were obtained by measuring on-peak for $100 \times 8 \mathrm{~s}$ integrations prior to each analysis. A washout time of ca. 30 min was applied between analyses and baseline measurements. Using this approach, background levels were typically $<<1 \mathrm{mV}$ on all $\mathrm{Pt}$ isotopes.

\section{Thermo Neptune Plus MC-ICPMS (DK)}

The Neptune MC-ICPMS was operated in low-resolution mode, with a mass resolution of $\sim 1600$ ( $\mathrm{M} / \Delta \mathrm{M}$ as defined by the peak edge width from $5-95 \%$ full peak height). An ESI Apex IR desolvating nebuliser sample introduction system was used without nitrogen input and with an Actively Cooled Membrane (ACM) add-on module for additional membrane desolvation. The sample uptake rate was ca. $30 \mu \mathrm{L} \mathrm{min}{ }^{-1}$, and the sensitivity of the instrument under these conditions was typically $\sim 100 \mathrm{~V} \mathrm{ppm}^{-1}$ total Pt. Solutions were prepared with a natural Pt concentration of $100 \mathrm{ng} \mathrm{mL}^{-1}$ and an almost equivalent additional Pt contribution from DS. Peak centering was performed at the start of each analysis, and each analysis comprised $100 \mathrm{x}$ $8.4 \mathrm{~s}$ integrations. Each analysis corresponded to $14 \mathrm{~min}$ of data acquisition and consumed ca. $40 \mathrm{ng}$ of natural Pt. Baselines were obtained by on-peak measurements for 100 x $8.4 \mathrm{~s}$ integrations prior to and following each analysis. A washout time of 30 min was used between sample analyses and baseline measurements. Using this approach, background levels were typically $<1 \mathrm{mV}$ 


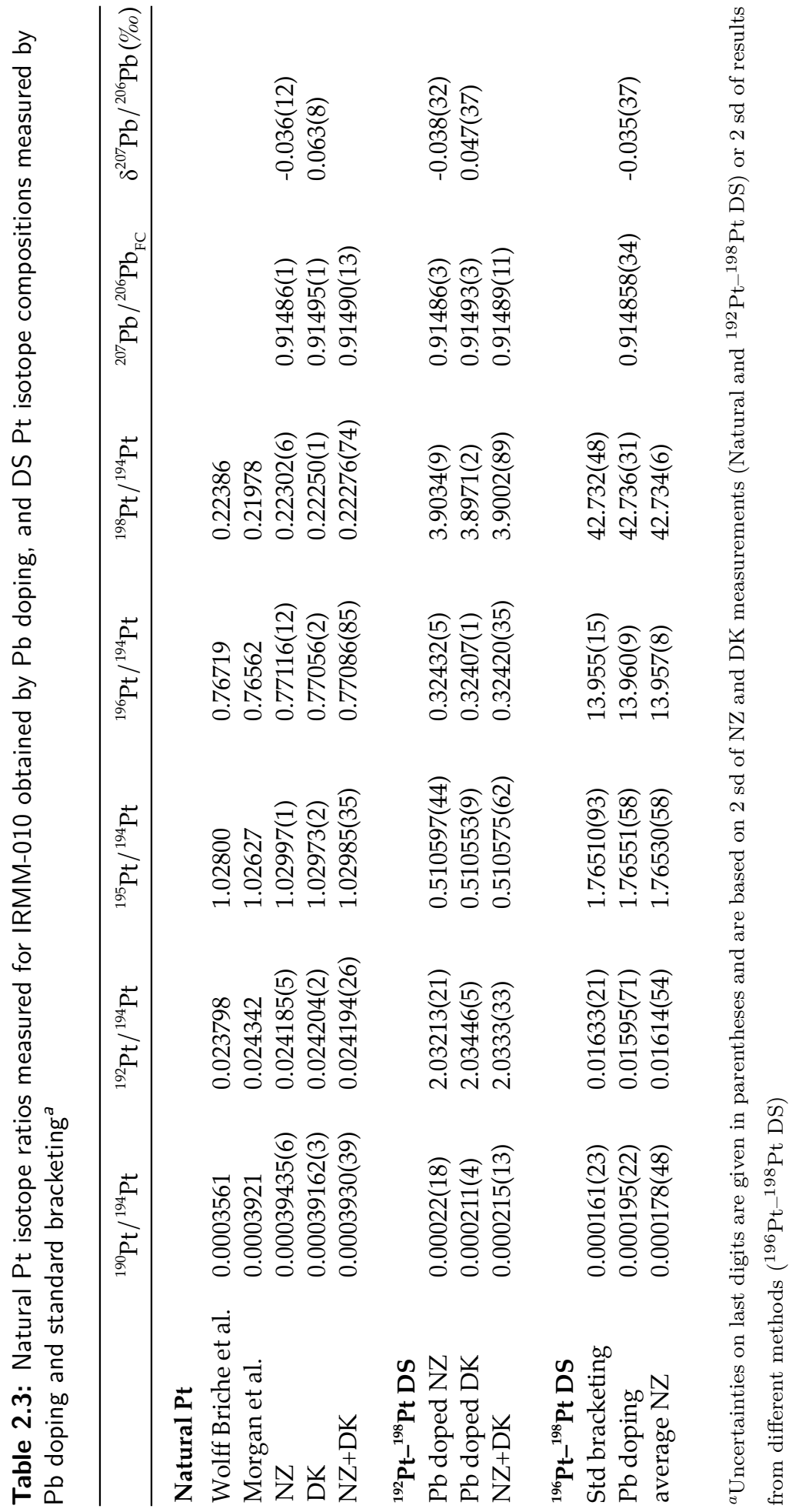


for all Pt isotopes.

\subsubsection{Interference correction}

Isobaric interferences on Pt exist from Os (masses 190 and 192) and $\mathrm{Hg}$ (masses 196 and 198). These overlapping masses, as well as most relevant molecular species, have mass differences that are too small to be resolved at the mass resolving capability of the MC-ICPMS instruments used in this study (Table 2.5). Contributions from $\mathrm{Os}$ and $\mathrm{Hg}$ were monitored using interference-free masses ${ }^{188} \mathrm{Os}$ and ${ }^{200} \mathrm{Hg}$. Due to its extremely volatile nature, interferences from $\mathrm{Hg}$ in natural samples are unlikely to be significant. However, due to the high relative abundances of ${ }^{190}$ Os and ${ }^{192}$ Os $(26.4 \%$ and $41 \%$, respectively), $\mathrm{Pt}$ isotope analyses at these masses are likely to be severely impacted by the presence of even small amounts of Os. In all of the undoped analyses presented here, signals on monitored $\mathrm{Os}$ and $\mathrm{Hg}$ masses were negligible (i.e. $<15 \mu \mathrm{V}$ ). An interference correction procedure for Os based on Scherstén et al. (2006) was tested. The interference-free ${ }^{189} \mathrm{Os} /{ }^{188}$ Os ratio (1.21967; Shirey and Walker, 1998) was used to calculate the Os mass bias, which was then used to calculate the Os interference corrections at masses 190 and 192 from the ${ }^{188}$ Os signal intensity.

In all of the undoped analyses presented here, signals on monitored Os and $\mathrm{Hg}$ masses were indistinguishable from the baseline noise.

\subsubsection{Data reduction}

All data reduction was conducted off-line using Iolite, a freely available data reduction package that runs within the Igor Pro software from Wavemetrics (Paton et al., 2011). An add-on has been developed for processing DS data within Iolite (Creech and Paul, 2014), which can be adapted for any DS system. This procedure will be made freely available for download from the Iolite website (www.iolite.org.au). The DS calculations are performed algebraically using the equations of Rudge et al. (2009). 


\subsubsection{Reporting of platinum stable isotope data}

In non-traditional stable isotope systems, particularly in the intermediateto heavy-mass range, it is conventional to report isotopic compositions as a ratio of a heavy over a light isotope for a given element (e.g. ${ }^{44} \mathrm{Ca} /{ }^{40} \mathrm{Ca}$ ), as is the case for traditional stable isotope systems (Johnson et al., 2004). We report results here using that approach. Given that the abundances of the two lightest $\mathrm{Pt}$ isotopes $\left({ }^{190} \mathrm{Pt}\right.$ and $\left.{ }^{192} \mathrm{Pt}\right)$ are very small, we use ${ }^{194} \mathrm{Pt}$ as the denominator isotope. Typically, in stable isotope studies, the highest mass isotope of a reasonably high abundance would be chosen for the numerator isotope. Therefore, we report $\mathrm{Pt}$ stable isotopes in terms of ${ }^{198} \mathrm{Pt} /{ }^{194} \mathrm{Pt}$ ratios. Given that mass-dependent natural stable isotope fractionations in Pt isotopes might be expected to be relatively small (i.e. parts-per-million), we have adopted a $\mu$ notation representing the parts per million deviation of the ${ }^{198} \mathrm{Pt} /{ }^{194} \mathrm{Pt}$ ratio from the same ratio for the IRMM-010 $\mathrm{Pt}$ isotope standard. Isotopic compositions are thus reported as $\mu^{198} \mathrm{Pt}$ :

$$
\mu^{198} P t=\left[\frac{\frac{198 P t}{194} \text { Pt sample }}{{ }^{198} P t}-1\right] \times 10^{6}
$$

Assuming fractionation occurs through a mass-dependent process, stable isotope fractionations may also be expressed in terms of the relative difference per unified atomic mass unit (i.e. $\mathrm{ppm} \mathrm{u}^{-1}$ ).

\subsubsection{Analytical tests}

We conducted the following tests to demonstrate the veracity of our technique: (1) mixtures of the IRMM-010 and both DS were measured over a range of different mixing proportions to test the accuracy of the DS calibrations; (2) replicate DS measurements of the IRMM-010 standard were made over many months to assess both the within-session and long-term reproducibility of the technique; (3) a range of element doping tests were conducted to test the sensitivity of the technique to the presence of contaminants (par- 


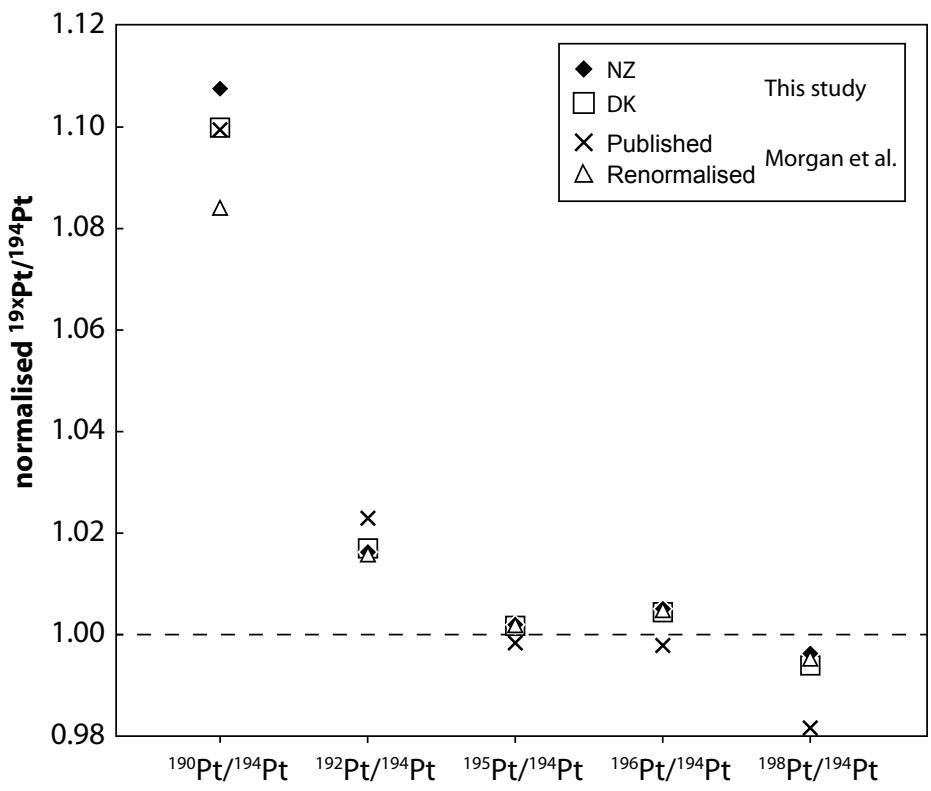

Figure 2.1: Natural Pt isotopic composition from Morgan et al. (2002) and $\mathrm{Pb}$-mass-bias-corrected MC-ICPMS analysis of the IRMM-010 standard in NZ and DK from this study normalised to the reference composition of Wolff Briche et al. (2002). Morgan et al. data represent published data internally normalised using ${ }^{194} \mathrm{Pt} /{ }^{195} \mathrm{Pt}=0.9744$ (crosses), and the same data renormalised to ${ }^{194} \mathrm{Pt} /{ }^{195} \mathrm{Pt}=0.9710$ as determined for natural $\mathrm{Pt}$ in this study (triangles).

ticularly Os) that may be present, for example, following chemical separation of Pt from natural samples; (4) the potential effects arising from imperfect peak positioning or lower ion beam intensities were examined by analysis off the peak centre and in low Pt concentration solutions; (5) Pt stable isotope compositions of three different Pt standards were measured to investigate whether any variability in Pt stable isotope compositions exists. 

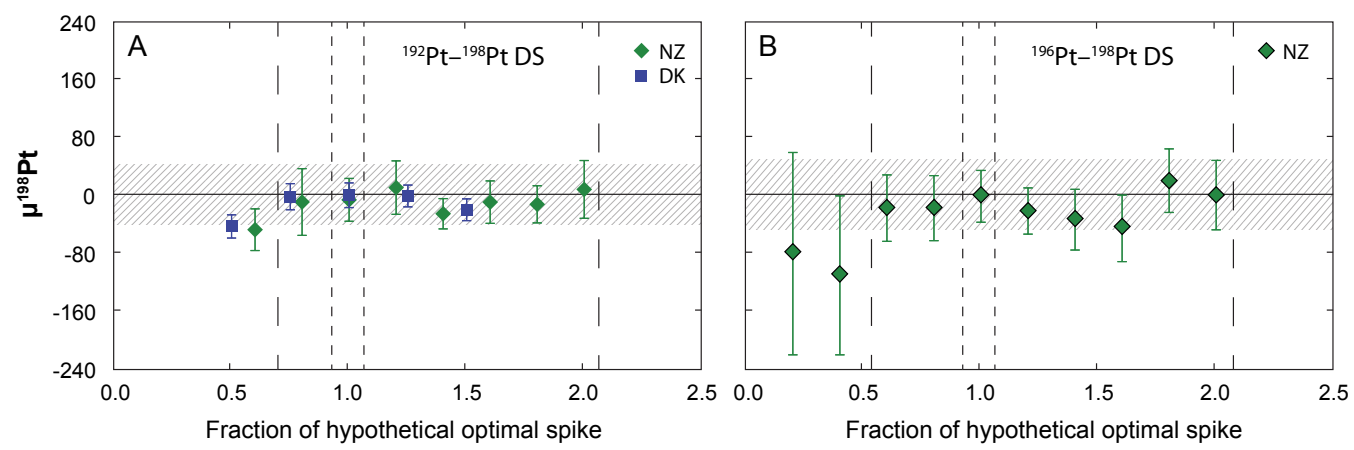

Figure 2.2: Plot of $\mu^{198} \mathrm{Pt}$ for a range of sample-spike mixtures expressed as a proportion of the hypothetical optimal sample-spike mixture. (A) Results for the ${ }^{192} \mathrm{Pt}-{ }^{198} \mathrm{Pt}$ double-spike from both NZ and DK. (B) Results for the ${ }^{196} \mathrm{Pt}-{ }^{198} \mathrm{Pt}$ double-spike from NZ only. These results demonstrate that $\mathrm{DS}$-corrected stable $\mathrm{Pt}$ isotope ratios are relatively insensitive to the sample-spike ratio. Coarse-dashed vertical lines represent the minimum range over which the calibration appears to be accurate. Fine-dashed vertical lines represent the approximate range of sample-spike mixtures used in acquiring the data presented elsewhere in this paper.

\subsection{Results and Discussion}

\subsubsection{Platinum isotopic composition of the IRMM-010 standard}

The currently accepted reference Pt isotope composition for IRMM-010 was determined using partially calibrated analyses by quadrupole ICPMS in 2002 (Wolff Briche et al., 2002). However, Pt isotope ratios reported by Morgan et al. (2002) measured by MC-ICPMS show significant differences in isotopic composition for some $\mathrm{Pt}$ isotopes as compared with those of Wolff Briche et al. (2002). During this study, it was found that no DS calibration would yield consistent DS-corrected isotope ratios using the natural Pt isotopic composition reported by Wolff Briche et al. (2002), suggesting that the reference values for IRMM-010 may be inaccurate. Therefore, we elected to remeasure the isotopic composition of IRMM-010 by MC-ICPMS. Platinum isotope measurements of IRMM-010 were carried out using external normalisation to the $\mathrm{Pb}$ isotope standard SRM981. Lead was selected for performing the mass bias correction as it is the closest element in mass to $\mathrm{Pt}$ that does not 
have any overlapping isobaric interferences on $\mathrm{Pt}$, and $\mathrm{Pb}$ isotopic reference standards with well constrained isotope ratios are available. Solutions of IRMM-010 were doped with the $\mathrm{Pb}$ isotope standard SRM981 at ca. 10\% of the Pt concentration and an internal mass bias correction was performed using a ${ }^{208} \mathrm{~Pb} /{ }^{206} \mathrm{~Pb}$ ratio of 2.16775 (Baker et al., 2004). This ${ }^{208} \mathrm{~Pb} /{ }^{206} \mathrm{~Pb}$ ratio for SRM981 was determined using the $\mathrm{Pb}$ DS approach referenced to a ${ }^{208} \mathrm{~Pb} /{ }^{206} \mathrm{~Pb}$ ratio of 1.00016 for the $\mathrm{Pb}$ isotope standard SRM982. The mass-bias-corrected ${ }^{207} \mathrm{~Pb} /{ }^{206} \mathrm{~Pb}$ ratio $\left({ }^{207} \mathrm{~Pb} /{ }^{206} \mathrm{~Pb}\right.$ FC $)$ was also measured and compared with the reference value of 0.91489 (Baker et al., 2004). The following factors must be taken into account when considering the accuracy of this approach to determining $\mathrm{Pt}$ isotope ratios:

(1) The potential presence of $\mathrm{Pt}$ oxide and nitride interferences on $\mathrm{Pb}$ isotopes (Table 2.5), which must either be insignificant or corrected for. To account for these potential interferences, measurements of $\mathrm{Pb}$-doped $\mathrm{Pt}$ standards were bracketed with analyses of an undoped Pt standard solution with an identical Pt concentration, and the signals observed in the undoped $\mathrm{Pt}$ standard at the $\mathrm{Pb}$ masses were subtracted from $\mathrm{Pb}$ doped analyses. The contributions on $\mathrm{Pb}$ masses from interferences were ca. $1 \mathrm{mV}$ for undoped $150 \mathrm{ppb}$ IRMM-010 solutions, compared with signals of $2-5 \mathrm{~V}$ in $\mathrm{Pb}$ doped analyses.

(2) That the relative mass bias behaviour of $\mathrm{Pb}$ and $\mathrm{Pt}$ are similar. Although the mass bias behaviour is unlikely to be identical for $\mathrm{Pt}$ and $\mathrm{Pb}$, this assumption is still sufficient to explore and further refine the Pt isotopic composition of IRMM-010 given that the studies of Wolff Briche et al. (2002) and Morgan et al. (2002) yield differences of 1-10\% in most Pt isotopic ratios.

(3) The accuracy of the $\mathrm{Pb}$-corrected $\mathrm{Pt}$ isotope ratios are dependent on the reference values for the $\mathrm{Pb}$ isotope standard SRM981 and, in turn, SRM982 which are considered to be accurate to $\pm 0.036 \%$ Catanzaro et al. 1968).

$\mathrm{Pb}$-corrected $\mathrm{Pt}$ isotope ratio measurements using the approach described above are shown in Table 2.3 . For all these measurements, ${ }^{207} \mathrm{~Pb} /{ }^{206} \mathrm{~Pb} \mathrm{FC}_{\mathrm{F}}$ 
ratios were within ca. $65 \mathrm{ppm}$ of the SRM981 reference value. Measured $\mathrm{Pt}$ isotope ratios are shown in Fig. 2.1 normalised to the reference composition from Wolff Briche et al. (2002). The most significant offsets in our data from the values of Wolff Briche et al. (2002) are for the minor isotope ratios ${ }^{190} \mathrm{Pt} /{ }^{194} \mathrm{Pt}$ and ${ }^{192} \mathrm{Pt} /{ }^{194} \mathrm{Pt}$, which show differences of ca. $10 \%$ and $1.7 \%$, respectively. Corrected Pt isotope ratios are in good agreement between the two instruments used in this study, with relative differences of $0.02 \%-0.2 \%$. For all ratios, measured IRMM-010 Pt isotopic compositions from the two instruments in this study are in significantly better agreement with one another and with those of Morgan et al. (2002) than they are with those of Wolff Briche et al. (2002). We have thus adopted the mean of the ratios from NZ and DK in this study as the composition of the IRMM-010 standard.

The analytical uncertainties associated with the $\mathrm{Pb}$-corrected $\mathrm{Pt}$ isotope ratios are relatively small compared with the differences from the reference composition (Wolff Briche et al., 2002). Estimated combined uncertainties were calculated for corrected ratios according to the uncertainty propagation law (jcg, Williams, 2010). However, the propagated uncertainties are significantly smaller than the variance between NZ and DK Pt isotope measurements. We have therefore conservatively adopted the 2 sd of the combined $\mathrm{NZ}$ and DK values as the uncertainty in the mean of the natural Pt isotope ratios.

Although the Pt isotope ratios measured in NZ and DK exhibit a closer agreement with one another than those of Wolff Briche et al. (2002), differences between the ratios measured in NZ and DK exceed the internal precision of the isotope ratio measurements by a factor of ca. $2-8$. The differences are greatest for the ratio involving the minor ${ }^{190} \mathrm{Pt}$ isotope and for ratios where the relative mass difference between the numerator and denominator isotope is highest (i.e., ${ }^{198} \mathrm{Pt} /{ }^{194} \mathrm{Pt}$ ). The cause of this discrepancy is not entirely clear. The abundance sensitivity of both of the mass spectrometers used in this study is sufficiently low that tails from abundant Pt isotopes 

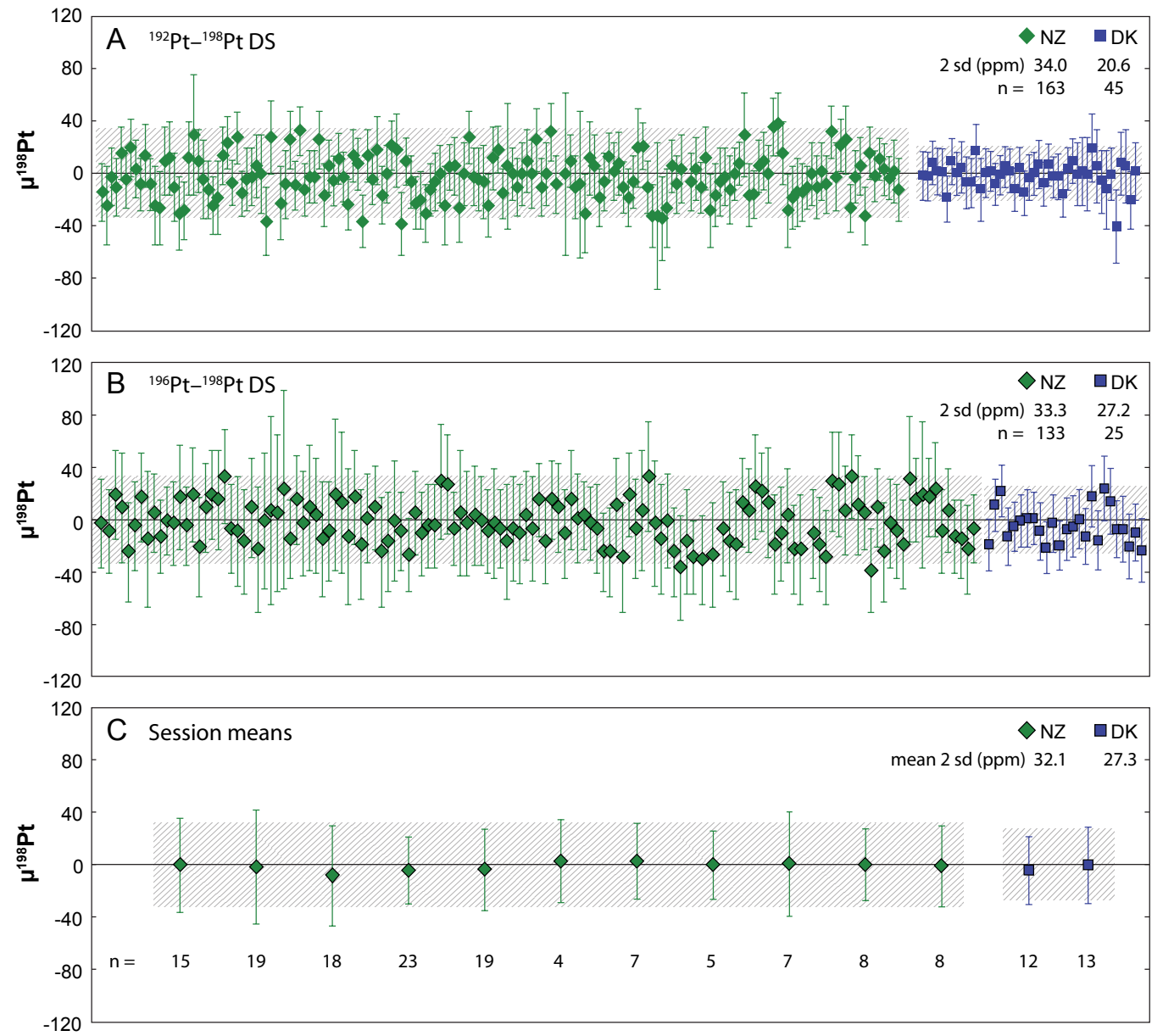

Figure 2.3: Reproducibility of double-spiked MC-ICPMS stable Pt isotope measurements from analytical sessions over a period of several months. The first two panels show all analyses from sessions using the ${ }^{192} \mathrm{Pt}-{ }^{198} \mathrm{Pt}(\mathrm{A})$ and ${ }^{196} \mathrm{Pt}-{ }^{198} \mathrm{Pt}(\mathrm{B})$ double-spikes, and error bars representing the 2 se error for each analysis. Hatched fields represent the \pm 2 sd of all analyses. The bottom panel (C) shows the mean and 2 sd (error bars) for each analytical session for ${ }^{196} \mathrm{Pt}-{ }^{198} \mathrm{Pt}$ DS data, which is our preferred double-spike. The number of analyses in each session is shown. Hatched fields represent the combined \pm 2 sd of all sessions from each laboratory.

onto less abundant Pt isotopes cannot readily explain the difference. Similarly, the opposite offsets observed for ${ }^{198} \mathrm{Pt} /{ }^{194} \mathrm{Pt}$ and ${ }^{192} \mathrm{Pt} /{ }^{194} \mathrm{Pt}$ between the two laboratories cannot be attributed to contamination or memory from use of the ${ }^{192} \mathrm{Pt}-{ }^{198} \mathrm{Pt}$ DS (the natural measurements were made prior to development of the $\left.{ }^{196} \mathrm{Pt}-{ }^{198} \mathrm{Pt} \mathrm{DS}\right)$. The difference in measured ${ }^{190} \mathrm{Pt} /{ }^{194} \mathrm{Pt}$ 
$(0.7 \%)$ obtained in the two laboratories is most likely a result of the very small ion beam size of the ${ }^{190} \mathrm{Pt}$ isotope (ca. $3 \mathrm{mV}$ ). The differences in the other Pt isotope ratios appear to broadly correspond to a mass fractionation relationship as the two sets of ratios lie within $40-180 \mathrm{ppm}$ of a mass fractionation line that passes through the mean composition of both sets of data, implying that subtle differences in the relative mass bias of $\mathrm{Pt}$ and $\mathrm{Pb}$ characterised the two mass spectrometers used in this study.

Relative Pt isotope abundances were calculated from the mean NZ-DK Pt isotope ratios in Table 2.3. Uncertainties in the abundances were calculated by partitioning ratio uncertainties between the numerator and denominator isotope following an error propagation law and using measured ion beam intensities (Williams, 2010). Given that the IRMM-010 Pt isotope standard is accepted as a reference for the natural composition of Pt (Berglund and Wieser, 2011), the new composition for IRMM-010 presented here should also be representative of natural $\mathrm{Pt}$. Our Pt isotopic composition is compared with previously published values for the natural composition of Pt in Table 2.1

We have also recalculated the atomic weight of Pt from the new IRMM010 isotopic composition presented here using the isotopic masses of Audi et al. (2003). We obtain an atomic weight for Pt of $195.08395 \pm 0.00068$. Despite the significant differences in the Pt isotopic composition reported in this study and (Wolff Briche et al., 2002), this new atomic weight is very similar to the value of $195.0844 \pm 0.0058$ from (Wolff Briche et al. 2002 ), although the uncertainty of our new value is significantly smaller than previous estimates (Table 2.1).

\subsubsection{Calibration of the platinum double-spikes}

Accurate isotopic determination by the DS technique requires calibration of the DS composition. Several approaches for calibrating the DS are possible. Here, we determined the compositions of the two DS in two ways: (1) externally normalised by doping with $\mathrm{Pb}$ in the same way as described above for 
the natural Pt isotopic composition of IRMM-010; and (2) external standard-spike bracketing versus IRMM-010. Isotopic abundances for both of the Pt DS used in this study are shown in Table 2.1 and expressed as ratios in Table 2.3. The calibrated DS compositions are not identical to the optimal DS from Rudge et al. (2009), which is due to subtle differences in the pure spike compositions from those used in the DS toolbox (Table 2.1).

\section{${ }^{192} \mathrm{Pt}-{ }^{198} \mathrm{Pt}$ double-spike}

In the case of the ${ }^{192} \mathrm{Pt}-{ }^{198} \mathrm{Pt} \mathrm{DS}, \mathrm{Pt}$-based oxide and nitride interferences on $\mathrm{Pb}$ isotopes were more significant than for IRMM-010 due to the higher abundance of ${ }^{192} \mathrm{Pt}$. Potential levels of interferences on $\mathrm{Pb}$ isotopes were monitored in undoped Pt DS solutions with identical Pt concentrations to the SRM981-Pt DS mixture and were subtracted from Pb-doped analyses. $\mathrm{Pb}$-doped measurements of the ${ }^{192} \mathrm{Pt}-{ }^{198} \mathrm{Pt}$ DS were repeated in NZ and DK. Pb-corrected ${ }^{207} \mathrm{~Pb} /{ }^{206} \mathrm{~Pb}_{\mathrm{FC}}$ ratios in both $\mathrm{NZ}$ and $\mathrm{DK}$ measurements were within ca. $50 \mathrm{ppm}$ of the reference value for SRM981. Pt isotopic compositions of this DS measured in NZ and DK are in good agreement, and yielded mean ratios of ${ }^{192} \mathrm{Pt} /{ }^{194} \mathrm{Pt}=2.0333 \pm 0.0033,{ }^{196} \mathrm{Pt} /{ }^{194} \mathrm{Pt}=0.32420$ \pm 0.00035 and ${ }^{198} \mathrm{Pt} /{ }^{194} \mathrm{Pt}=3.9002 \pm 0.0089$ for the three ratios used in the DS calculations, where the uncertainties represent the $2 \mathrm{sd}$ of the NZ and DK isotope measurements (Table 2.3).

\section{${ }^{196} \mathrm{Pt}-{ }^{198} \mathrm{Pt}$ double-spike}

The ${ }^{196} \mathrm{Pt}-{ }^{198} \mathrm{Pt}$ DS composition was determined by both $\mathrm{Pb}$ doping and IRMM-010 standard-spike bracketing, although these measurements were only carried out in NZ. Both sets of results are very consistent, with compositions agreeing to within $0.04 \%$. The $\mathrm{Pt}$ isotope ratios obtained for the ${ }^{196} \mathrm{Pt}-{ }^{198} \mathrm{Pt}$ DS were ${ }^{195} \mathrm{Pt} /{ }^{194} \mathrm{Pt}=1.76530 \pm 0.00058,{ }^{196} \mathrm{Pt} /{ }^{194} \mathrm{Pt}=13.957$ 
\pm 0.008 and ${ }^{198} \mathrm{Pt} /{ }^{194} \mathrm{Pt}=42.734 \pm 0.006$, where the uncertainties represent the $2 \mathrm{sd}$ of the ratios obtained by the two different methods.

\section{Accuracy of the double-spike calibrations}

The accuracy of the DS calibrations was tested by measuring DS-IRMM-010 mixtures in different proportions. DS-corrected results for 8-10 calibration mixtures for both DS, where the mixtures were varied from $0.2-2.0$ times the approximate 'optimal' sample-spike ratio are shown in Fig. 2.2. Varying the sample/spike ratios from $\sim 0.6-2.0$ times the optimal sample-spike proportion for either DS has no influence on the accuracy of the DS correction, indicating that the two DS have been well calibrated and that a relatively wide window is acceptable for measuring sample-DS mixtures without significant degradation of the results.

\subsubsection{Internal precision and external reproducibility of double-spike-corrected platinum stable isotope ratios}

Internal precision on DS-corrected $\mathrm{Pt}$ isotope ratios varied slightly from session-to-session depending on the stability of the sample introduction systems. However, typical internal $(2 \mathrm{se})$ precision on $\mu^{198} \mathrm{Pt}$ was ca. $\pm 30 \mathrm{ppm}$ and $\pm 16 \mathrm{ppm}$ for NZ and DK analyses, respectively. The improved internal precision in DK compared with NZ mainly reflects the greater stability of the Apex sample introduction system.

DS-corrected Pt isotope results for IRMM-010 from a number of analytical sessions in both NZ and DK and using both DS are shown in Fig. 2.3. The external reproducibilities using the two DS are very similar. Within measurement sessions that are typically 1-2 days in length, DS-corrected IRMM-010 measurements have external reproducibilities $(2 \mathrm{sd})$ on $\mu^{198} \mathrm{Pt}$ of ca. $\pm 35 \mathrm{ppm}$ for $\mathrm{NZ}$ analyses and ca. $\pm 25 \mathrm{ppm}$ for DK analyses. External 


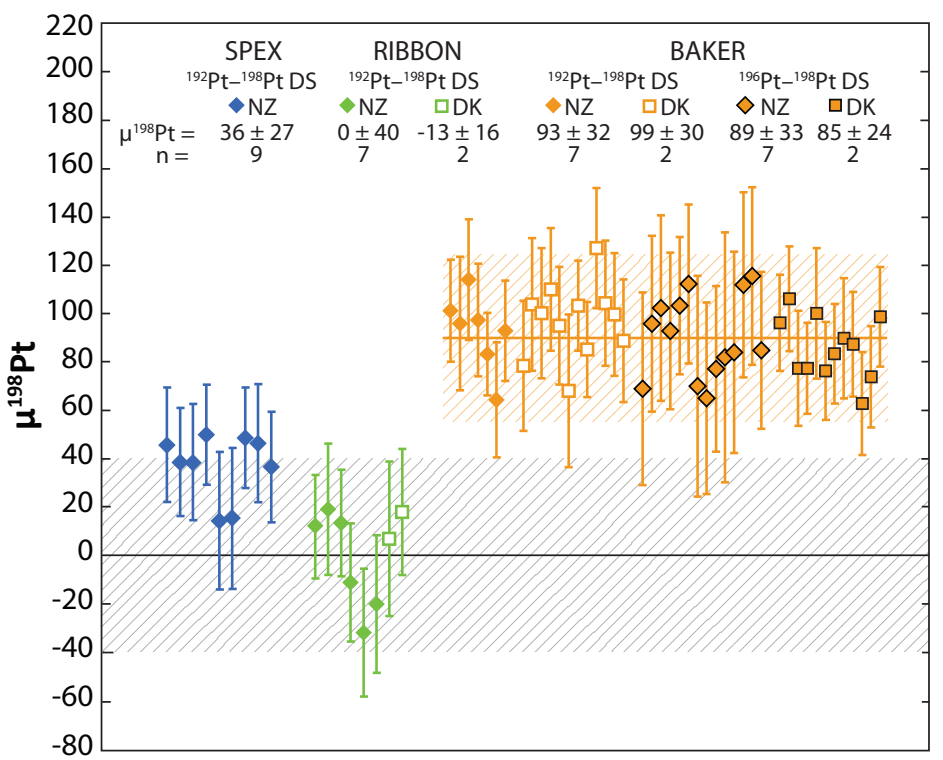

Figure 2.4: Measurements of $\mu^{198} \mathrm{Pt}$ in the additional Pt standards described in the text. The Ribbon standard is compositionally identical to the IRMM-010 standard. The Spex standard shows a potential offset from the IRMM-010 composition, although the offset is approximately equal to the reproducibility of the technique. However, the Baker standard show a clear offset from the IRMM-010 standard of $89 \pm 33 \mathrm{ppm}$ that is reproducible between laboratories and using both types of DS. Grey hatched area represents the reproducibility of the technique. Orange horizontal line and hatched area represent the mean and 2 sd, respectively, of all analyses of the Baker standard.

reproducibility over a large number of sessions is similar to reproducibility attained in a single session (Fig. 2.3).

Small offsets (ca. 30-50 ppm) were sometimes observed between absolute DS-corrected Pt isotope ratios from different sessions. These offsets might possibly be related to small changes in the abundance sensitivity of the MCICPMS, drifts in the relative gains of the Faraday collectors, or a variable component of non-exponential mass bias that is not readily corrected for by the DS method. To account for these variations, we combined the DS correction method with a standard-sample bracketing approach, whereby analyses of the double-spiked standard are repeated throughout the session and alternated with "unknown" analyses. The analyses of "unknowns" are then normalised to the mean value of the bracketing standards. The long- 
term external reproducibility of these corrected ratios is approximately the same as the within-session reproducibility. The external reproducibility of our technique suggests that mass-dependent Pt stable isotope fractionations $>10 \mathrm{ppm} \mathrm{u}^{-1}$ should be resolvable by this technique. This external reproducibility is comparable to those recently achieved for other non-traditional stable isotope systems such as $\mathrm{Mg}$, Fe and Cd (e.g. Bizzarro et al., 2011. Millet et al., 2012, Schmitt et al., 2009).

\section{Platinum stable isotope variations in standards}

Repeated DS-corrected Pt stable isotope measurements were made of three different Pt element standards (Baker, Ribbon and Spex; Table 2.3. Fig. 2.4. ${ }^{192} \mathrm{Pt}-{ }^{198} \mathrm{Pt}$ DS-corrected measurements were made of all three standards in both NZ and DK. Measurements using the ${ }^{196} \mathrm{Pt}-{ }^{198} \mathrm{Pt}$ DS were only repeated in both laboratories for the Baker standard. The Spex standard may show a subtle variation from the IRMM-010 standard, however, both Spex and Ribbon are within error of the IRMM-010 composition. The Baker standard shows the largest offset in $\mu^{198} \mathrm{Pt}$ of ca. $89 \pm 33 \mathrm{ppm}$ from IRMM010, which was reproducibly observed between different analytical sessions in both NZ and DK and using both DS. The Baker standard thus represents a useful secondary standard with a known offset from IRMM-010 to monitor the quality of $\mathrm{Pt}$ isotope data within each analytical session. Moreover, these stable Pt isotopic differences between the IRMM-010 and Baker standards suggest that stable isotopic fractionations characterise terrestrial $\mathrm{Pt}$, although it is currently unclear if this is due to variations in the natural $\mathrm{Pt}$ source material or those induced during preparation of the Pt metal used to make these standards. 
Table 2.4: Results of elemental doping tests to assess potential matrix effects. Optimally spiked IRMM-010 samples were doped with a range of elements.

\begin{tabular}{|c|c|c|c|c|c|c|c|}
\hline \multirow[b]{2}{*}{ Dopant } & \multirow{2}{*}{$\begin{array}{l}\text { Doping } \\
\text { level }\end{array}$} & \multicolumn{3}{|c|}{${ }^{192} \mathrm{Pt}-{ }^{198} \mathrm{Pt}$ DS } & \multicolumn{3}{|c|}{${ }^{196} \mathrm{Pt}-{ }^{198} \mathrm{Pt} \mathrm{DS}$} \\
\hline & & $\mu^{198} \mathrm{Pt}$ & $2 \mathrm{sd}$ & $n$ & $\mu^{198} \mathrm{Pt}$ & $2 \mathrm{sd}$ & $n$ \\
\hline Undoped & & 0.0 & 63.0 & 39 & 0.0 & 43.0 & 49 \\
\hline \multirow{2}{*}{$\mathrm{Mg}$} & $5 \%$ & -54.5 & 38.4 & 2 & -45.0 & 23.0 & 3 \\
\hline & $10 \%$ & -30.5 & 31.9 & 2 & -11.0 & 25.2 & 3 \\
\hline \multirow{2}{*}{$\mathrm{Al}$} & $5 \%$ & -31.0 & 86.5 & 3 & -31.3 & 31.3 & 3 \\
\hline & $10 \%$ & -70.8 & 72.1 & 4 & -8.7 & 30.9 & 3 \\
\hline \multirow{2}{*}{$\mathrm{Ca}$} & $5 \%$ & 9.0 & 32.6 & 2 & -37.3 & 22.3 & 3 \\
\hline & $10 \%$ & -50.0 & 31.8 & 2 & -5.7 & 24.9 & 3 \\
\hline \multirow{2}{*}{$\mathrm{Ti}$} & $5 \%$ & 82.0 & 35.1 & 2 & -25.0 & 34.2 & 3 \\
\hline & $10 \%$ & 58.5 & 31.6 & 2 & -14.7 & 23.0 & 3 \\
\hline \multirow{2}{*}{$\mathrm{Cr}$} & $5 \%$ & 18.0 & 63.0 & 1 & 7.0 & 21.6 & 3 \\
\hline & $10 \%$ & 90.0 & 63.0 & 1 & -2.0 & 26.4 & 3 \\
\hline \multirow{2}{*}{$\mathrm{Mn}$} & $5 \%$ & -18.0 & 45.3 & 2 & -12.3 & 24.0 & 3 \\
\hline & $10 \%$ & -6.0 & 38.0 & 2 & -6.7 & 27.4 & 3 \\
\hline \multirow{2}{*}{$\mathrm{Fe}$} & $5 \%$ & -11.3 & 48.6 & 6 & -11.3 & 59.2 & 3 \\
\hline & $10 \%$ & -8.8 & 53.9 & 6 & -20.7 & 22.4 & 3 \\
\hline \multirow{2}{*}{$\mathrm{Ni}$} & $5 \%$ & & & & 29.0 & 28.4 & 3 \\
\hline & $10 \%$ & -19.0 & 63.0 & 1 & 7.0 & 23.2 & 3 \\
\hline \multirow{2}{*}{$\mathrm{Cu}$} & $5 \%$ & & & & -17.3 & 23.4 & 3 \\
\hline & $10 \%$ & & & & -7.7 & 23.0 & 3 \\
\hline \multirow{2}{*}{ Mo } & $5 \%$ & & & & 1.0 & 29.9 & 3 \\
\hline & $10 \%$ & & & & -18.0 & 41.5 & 3 \\
\hline \multirow{2}{*}{ W } & $5 \%$ & & & & -13.3 & 25.4 & 3 \\
\hline & $10 \%$ & & & & 15.3 & 41.0 & 4 \\
\hline \multirow{2}{*}{$\mathrm{Rh}$} & $5 \%$ & -50.0 & 63.0 & 1 & -8.7 & 43.3 & 3 \\
\hline & $10 \%$ & 9.0 & 63.0 & 1 & 14.5 & 48.8 & 4 \\
\hline \multirow{2}{*}{$\mathrm{Pd}$} & $5 \%$ & -11.0 & 63.0 & 1 & -22.7 & 31.3 & 3 \\
\hline & $10 \%$ & -8.0 & 63.0 & 1 & 28.0 & 34.5 & 3 \\
\hline \multirow{2}{*}{ Ir } & $5 \%$ & -21.0 & 63.0 & 1 & -5.0 & 23.1 & 2 \\
\hline & $10 \%$ & -17.0 & 63.0 & 1 & 9.5 & 42.0 & 2 \\
\hline \multirow{2}{*}{$\mathrm{Au}$} & $5 \%$ & 20.0 & 63.0 & 1 & -2.3 & 25.5 & 3 \\
\hline & $10 \%$ & 1.0 & 63.0 & 1 & 48.0 & 25.5 & 3 \\
\hline \multirow{7}{*}{ Os } & $0.1 \%$ & -2.0 & 135.8 & 2 & & & \\
\hline & $0.2 \%$ & 15.0 & 65.1 & 2 & & & \\
\hline & $0.5 \%$ & 117.0 & 45.3 & 2 & -23.3 & 22.1 & 3 \\
\hline & $0.9 \%$ & 584.0 & 11.3 & 2 & -15.3 & 24.2 & 3 \\
\hline & $1.8 \%$ & 1063.0 & & 1 & & & \\
\hline & $4.5 \%$ & 3022.5 & 58.0 & 2 & 2.7 & 32.0 & 3 \\
\hline & $9.0 \%$ & & & & -24.0 & 34.2 & 3 \\
\hline
\end{tabular}

${ }^{a}$ Uncertainties are propagated 2 sd errors. Italicised 2 sd values are where only one analysis was made and the session $2 \mathrm{sd}$ is taken as the uncertainty. No Os interference correction for ${ }^{192} \mathrm{Pt}-{ }^{198} \mathrm{Pt} \mathrm{DS}$ data was applied. 

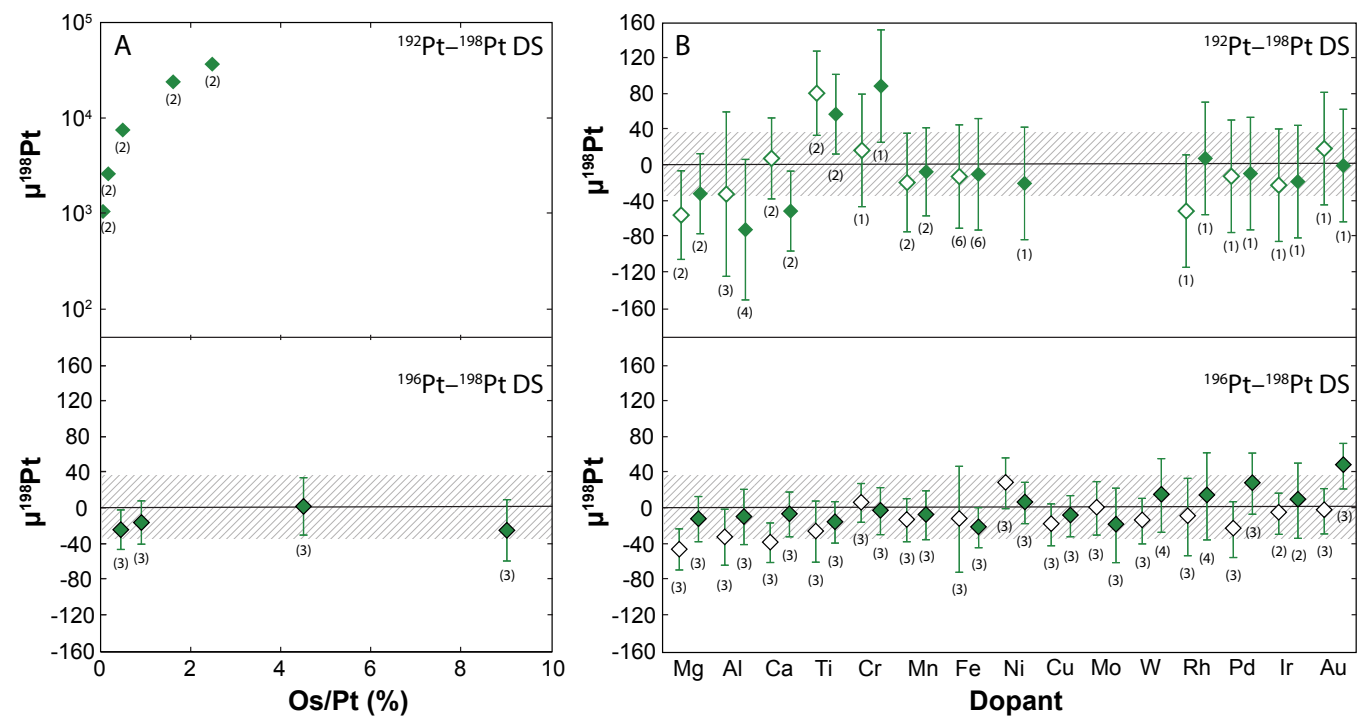

Figure 2.5: Results of doped IRMM-010 standard measurements with both double-spikes from NZ. (A) Results of doping tests with a range of Os concentrations up to $10 \%$ of the $\mathrm{Pt}$ concentration of the standard. The ${ }^{192} \mathrm{Pt}-{ }^{198} \mathrm{Pt}$ DS-corrected $\mu^{198} \mathrm{Pt}$ data are sensitive to very small amounts of Os contamination (note: data shown have no Os correction applied). However, the ${ }^{196} \mathrm{Pt}-{ }^{198} \mathrm{Pt}$ DS corrected $\mu{ }^{198} \mathrm{Pt}$ appears to be unaffected at the Os doping levels tested here. Hatched areas represent the 2 sd reproducibility of undoped measurements (not shown for the ${ }^{192} \mathrm{Pt}-{ }^{198} \mathrm{Pt} \mathrm{DS}$ ). (B) Results of doping tests with a range of common rock forming elements and siderophile elements for both double-spikes. Solutions of IRMM- 010 were doped at $5 \%$ and $10 \%$ of the Pt concentration, represented by hollow and filled symbols, respectively. Hatched areas represent the 2 sd reproducibility of undoped measurements for each DS.

\subsubsection{Analytical tests}

\section{Spectral matrix effects}

Due to the high relative abundances of ${ }^{190} \mathrm{Os}$ and ${ }^{192} \mathrm{Os}(26.4 \%$ and $41 \%$, respectively), Pt isotope analyses at these masses are likely to be severely impacted by the presence of even small amounts of Os, particularly where one of these two Pt isotopes would be used in the DS calculations. In order to constrain the potential effects of Os isobaric interferences, such as those that might result from imperfect chemical separation of $\mathrm{Pt}$ from Os in natural samples, Os doping tests were carried out on DS-IRMM-010 mixtures using 
both the ${ }^{192} \mathrm{Pt}-{ }^{198} \mathrm{Pt}$ DS and ${ }^{196} \mathrm{Pt}-{ }^{198} \mathrm{Pt}$ DS (Fig. 2.5A). Os doping levels ranged from $0.1-10 \%$ of the $\mathrm{Pt}$ concentration . The ${ }^{196} \mathrm{Pt}-{ }^{198} \mathrm{Pt}$ spike does not use ${ }^{192} \mathrm{Pt}$ in the DS calculations, and is unaffected by the presence of Os even at the highest doping levels. However, DS-corrected Pt isotope data using the ${ }^{192} \mathrm{Pt}-{ }^{198} \mathrm{Pt}$ DS are highly sensitive to the presence of small amounts of Os. At Os $/ \mathrm{Pt}$ ratios $>0.1 \%$, DS corrected $\mu^{198} \mathrm{Pt}$ analyses are inaccurate by $>1000$ ppm. The VUW Nu Plasma collector configuration does not allow for ${ }^{189} \mathrm{Os} /{ }^{188}$ Os to be measured in the same cycle as Pt isotopes, so this approach to calculating Os corrections on ${ }^{190} \mathrm{Pt}$ and ${ }^{192} \mathrm{Pt}$ was tested by measuring ${ }^{189} \mathrm{Os} /{ }^{188} \mathrm{Os}$ in a second cycle to determine the Os mass bias, and using ${ }^{188} \mathrm{Os}$ signal measured in the same cycle as $\mathrm{Pt}$ to calculate the magnitude of the corrections. While the Os correction was somewhat effective, it was found to be not sufficiently reliable to enable accurate isotope determinations using the ${ }^{192} \mathrm{Pt}-{ }^{198} \mathrm{Pt}$ DS in standards even with only very minor amounts of Os contamination (i.e. Os $/ \mathrm{Pt}<0.1 \%$ ).

The influence of Os in ${ }^{192} \mathrm{Pt}-{ }^{198} \mathrm{Pt}$ DS Pt stable isotope measurements may limit the application of this DS to natural samples where Os and Pt may have broadly similar concentrations. Whilst chemical separation techniques can be used to quantitatively separate Os from Pt, and the presence of Os can at least be monitored using non-overlapping masses (e.g., ${ }^{188} \mathrm{Os}$ ), it is difficult to rigorously correct for the presence of even minor amounts of Os. As such, the ${ }^{196} \mathrm{Pt}-{ }^{198} \mathrm{Pt}$ DS is our preferred Pt DS for use with terrestrial samples. Furthermore, a recent study has shown that, due to their relatively large thermal neutron capture cross sections, Pt isotope compositions can be significantly altered by neutron capture in iron meteorites with long cosmic ray exposure ages (Kruijer et al., 2013). These effects are significantly larger for ${ }^{192} \mathrm{Pt}$ than ${ }^{196} \mathrm{Pt}$. Therefore, the ${ }^{196} \mathrm{Pt}-{ }^{198} \mathrm{Pt}$ DS is also our preferred DS for use with extraterrestrial samples. 


\section{Non-spectral matrix effects}

Non-spectral matrix effects from elements that have no direct isobaric interferences with the element of interest can arise from a range of possible factors and can have an influence on instrumental mass bias Albarède and Beard (2004). In order to investigate potential non-spectral matrix effects, a range of elemental doping tests were performed whereby solutions of optimally double-spiked IRMM-010 were doped at levels of $5-10 \%$ of the Pt concentration, including a range of common rock-forming elements $(\mathrm{Mg}, \mathrm{Al}$, $\mathrm{Ca}, \mathrm{Ti}, \mathrm{Cr}, \mathrm{Mn}, \mathrm{Fe}, \mathrm{Ni}$ and $\mathrm{Cu}$ ) as well some siderophile elements (Mo, W, $\mathrm{Rh}, \mathrm{Pd}, \mathrm{Ir}$ and $\mathrm{Au}$ ). The results of these tests are shown in Fig. 2.5B. The ${ }^{192} \mathrm{Pt}-{ }^{198} \mathrm{Pt}$ DS-corrected data for IRMM-010 doped with this range of elements at levels of 5-10\% are largely within analytical uncertainty of undoped IRMM-010, although Ti, Cr and Al may have a moderate (ca. $<100$ ppm) effect on the accuracy of $\mathrm{Pt}$ stable isotope measurements using the ${ }^{192} \mathrm{Pt}-{ }^{198} \mathrm{Pt}$ double-spike (Table 2.4; Fig. 2.5B). Element-doped IRMM-010 measured using the ${ }^{196} \mathrm{Pt}-{ }^{198} \mathrm{Pt}$ DS yielded Pt stable isotope ratios that were almost all within analytical precision of undoped IRMM-010. However, IRMM-010 doped with $\mathrm{Au}$ yielded a mean $\mu^{198} \mathrm{Pt}$ of $48.0 \pm 27.5 \mathrm{ppm}$, which might reflect a small hydride interference $\left({ }^{197} \mathrm{Au}^{1} \mathrm{H}^{+}\right)$on ${ }^{198} \mathrm{Pt}$.

These elemental-doping tests show that for most elements tested, impurities of up to $10 \%$ of the Pt concentration do not significantly compromise the accuracy of the DS-corrected Pt isotope ratios. These results provide some constraints on the efficiency of chemical separation procedures that will be required to separate sufficiently pure Pt from natural samples for accurate $\mathrm{Pt}$ stable isotope analysis. 


\section{Effect of Pt signal intensity}

To test the influence of signal intensity on DS-corrected Pt stable isotope measurements, DS-IRMM-010 mixtures were measured at ca. 30\% and 3\% of the typical Pt concentration analysed in this study (i.e. $7 \mathrm{~V}$ and $0.8 \mathrm{~V}$ total Pt signal compared with $24 \mathrm{~V}$ at the typical Pt concentration; Fig. 2.6 A). At $7 \mathrm{~V}$ total $\mathrm{Pt}$ signal, the DS-corrected Pt stable isotope ratios were within error of those measured at optimal intensities, although the internal precision and external reproducibility were slightly higher (ca. $35 \mathrm{ppm}$ and $70 \mathrm{ppm}$, respectively). At $0.8 \mathrm{~V}$ total $\mathrm{Pt}$ signal, the internal precision and external reproducibility were much larger (ca. $150 \mathrm{ppm}$ and $240 \mathrm{ppm}$, respectively). These data show that it will be possible to measure the Pt stable isotopic composition $\left(\mu^{198} \mathrm{Pt}\right)$ of natural samples to $\pm 70 \mathrm{ppm}$ on sample of $25 \mathrm{ng}$ of $\mathrm{Pt}$, and to $\pm 240 \mathrm{ppm}$ on a sample of $5 \mathrm{ng}$ of $\mathrm{Pt}$.

\section{Effect of peak position}

When operated in low-resolution mode, the Nu Plasma MC-ICPMS produces flat-topped peaks that are ca. $0.39 \mathrm{u}$ wide (full width at half maximum) for Pt masses. We tested the potential effects on Pt stable isotope ratio measurements that might result from imperfect alignment of the Pt isotope peaks, peak centering, and peak flatness by measuring DS-corrected Pt isotope ratios on IRMM-010 in different peak measurement positions. Analyses were made by manually setting the axial mass to a range of positions up to 0.1 amu either side of the peak centre and performing analyses of IRMM-010 using the ${ }^{196} \mathrm{Pt}-{ }^{198} \mathrm{Pt}$ DS. These tests showed that DS-corrected Pt isotope ratios were within analytical precision of those at the peak centre even when positioned up to ca. $\pm 0.075 \mathrm{u}$ (ca. $\pm 25 \%$ of the plateau width) from the peak centre (Fig. 2.6B). This range is significantly broader than any peak drift that is likely to occur during an analytical session and, thus, subtle 

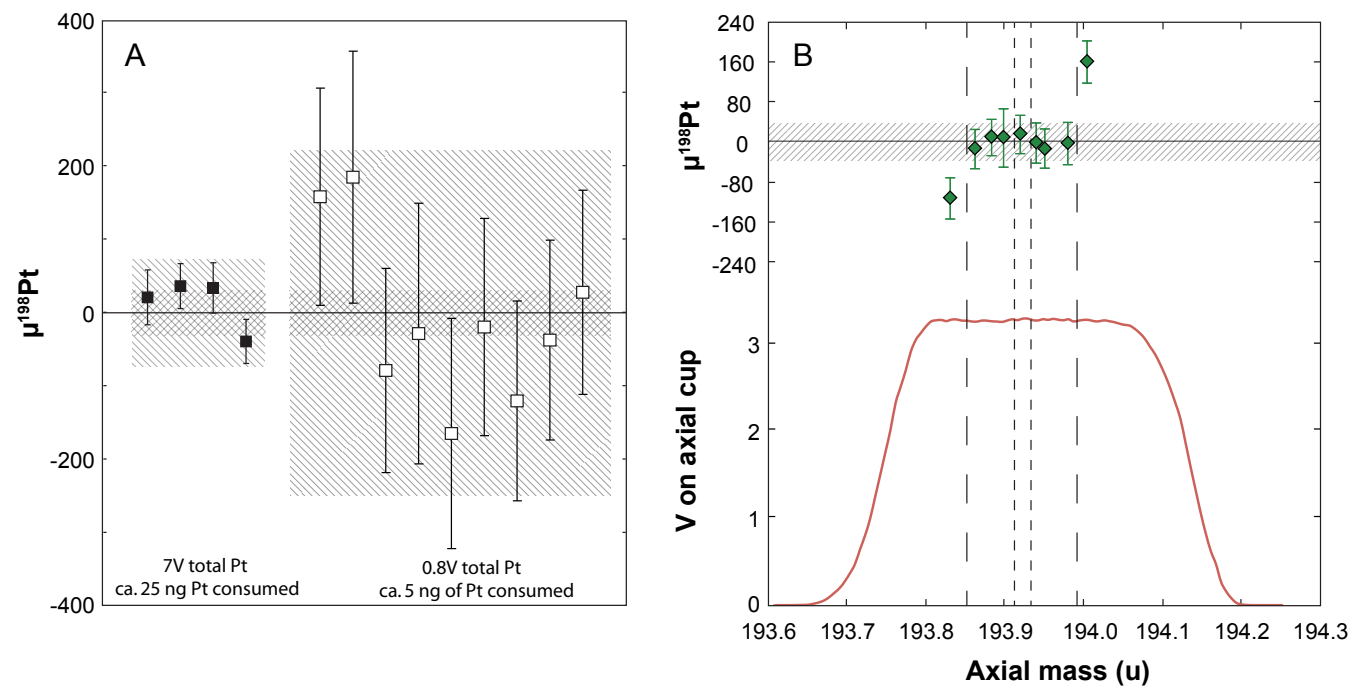

Figure 2.6: (A) Repeat measurements of IRMM-010 with the ${ }^{192} \mathrm{Pt}-{ }^{198} \mathrm{Pt}$ DS at $7 \mathrm{~V}$ (filled symbols) and ${ }^{196} \mathrm{Pt}-{ }^{198} \mathrm{Pt} \mathrm{DS}$ at $0.8 \mathrm{~V}$ total signal on $\mathrm{Pt}$ (usually $24 \mathrm{~V}$ at typical measurement concentration). Internal and external precision are concomitantly larger (ca. $35 \mathrm{ppm}$ and 73 $\mathrm{ppm}$, respectively, for $7 \mathrm{~V}$ signal, and ca. $150 \mathrm{ppm}$ and $240 \mathrm{ppm}$, respectively, for $0.8 \mathrm{~V}$ signal). Hatched area represents the 2 sd error for each group of samples. Cross-hatched areas represent the reproducibility of the technique using typical measurement concentrations. (B) Peak scan from the Nu Plasma MC-ICPMS in low resolution, with measurements of $\mu^{198} \mathrm{Pt}$ for IRMM-010 using the ${ }^{196} \mathrm{Pt}-{ }^{198} \mathrm{Pt}$ DS superimposed at their respective analysis peak positions (expressed in unified atomic mass units, u). The coarse dashed vertical lines represent the range over which the peak position had no effect. The fine dashed vertical lines represent the range of axial masses of normal measurements in this study. The hatched area represents the reproducibility of the technique.

changes in peak position are unlikely to have any effect on the accuracy of our measurements.

\subsection{Conclusions}

We have established a DS method for the precise measurement of Pt stable isotopes by MC-ICPMS using both ${ }^{192} \mathrm{Pt}-{ }^{198} \mathrm{Pt}$ and ${ }^{196} \mathrm{Pt}-{ }^{198} \mathrm{Pt}$ doublespikes. The latter spike is preferable for application to natural terrestrial and extraterrestrial samples as it is (relatively) insensitive to the presence of minor amounts of Os and any cosmogenic effects that can affect Pt isotopes 
in meteorite samples with long cosmic ray exposure ages (Kruijer et al., 2013 Wittig et al., 2013). Repeated measurements of Pt standards show that an external precision of ca. $\pm 10 \mathrm{ppm} \mathrm{u}^{-1}$ is routinely achievable using this technique, even in the presence of significant amounts of matrix elements. One of the analysed Pt standards clearly shows an offset from the IRMM-010 Pt isotope standard that suggest terrestrial stable Pt isotope variations exist that exceed our analytical uncertainty.

While Pt stable isotope variations in nature might be expect to be small, this new isotopic tracer may hold considerable promise for use in a range of areas, including; (1) Provenancing of global Pt sources and precious metal archeological artefacts; (2) Understanding the global Pt geochemical cycle and, in particular, using redox-controlled $\mathrm{Pt}$ stable isotope variations in marine authigenic sediments to trace and understand past changes in ocean and atmospheric chemistry; (3) Metal-silicate and liquid-solid metal fractionation associated with the differentiation of asteroids and terrestrial planets in the young Solar System.

\section{Acknowledgements}

We thank André Poirier for providing a segment of platinum ribbon, and Vickie Bennett for supplying additional PGE solutions. The authors also wish to thank H. Williams and one anonymous reviewer for their constructive comments on the manuscript. This research was supported by a Royal Society of New Zealand Marsden Grant to MH and JB. JC is funded by a VUW PhD scholarship. The Centre for Star and Planet Formation is funded by the Danish National Research Foundation and the University of Copenhagen's Programme of Excellence. 


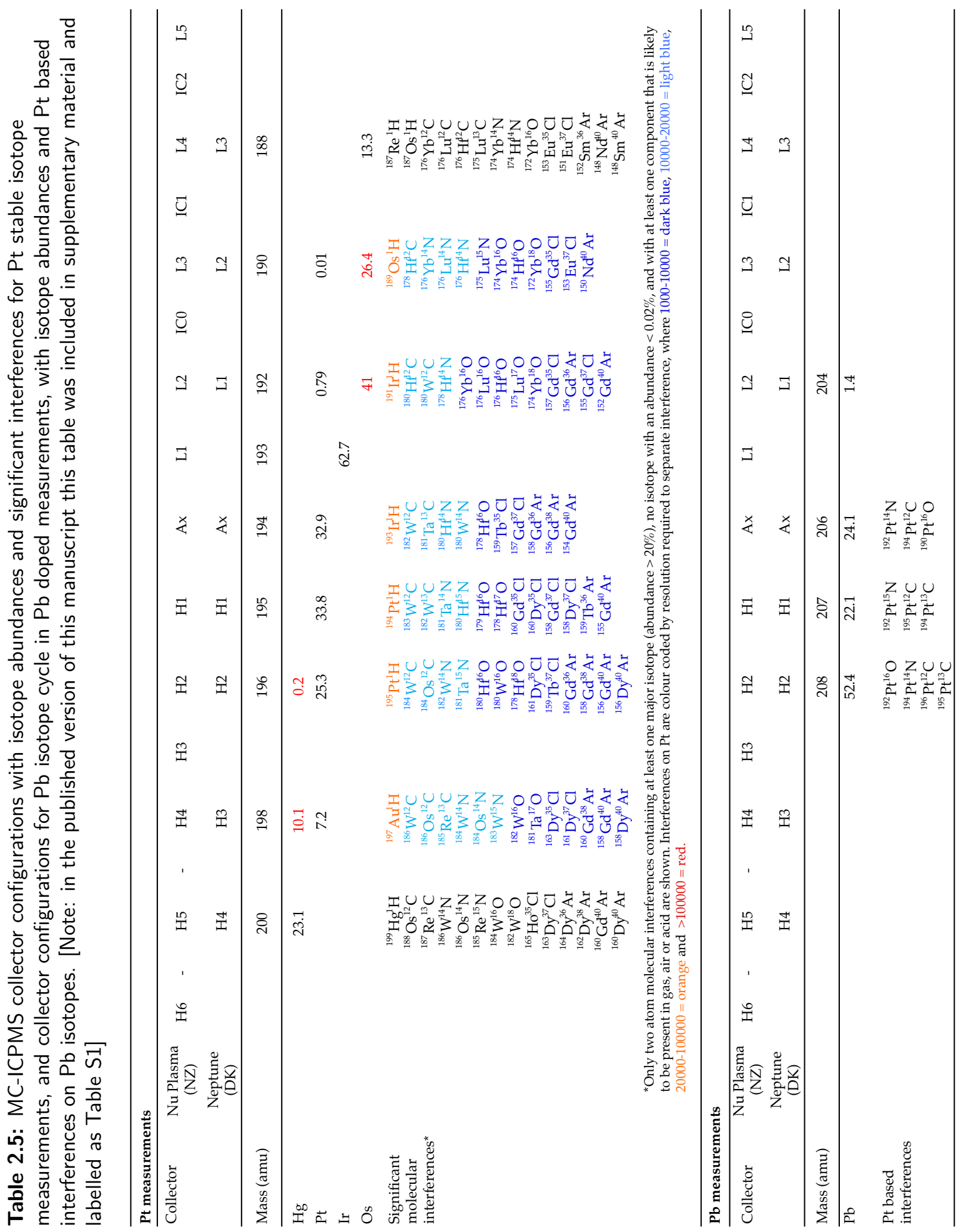




\section{Chapter 3}

\section{Chemical separation of $\mathrm{Pt}$}

This chapter was prepared as a journal article and is now published in Chemical Geology ${ }^{*}$. The article is entitled Platinum stable isotope analysis of geological standard reference materials by double spike MC-ICPMS. References have been gathered to the combined references section at the end of this thesis. A reprint of the published article is included as Appendix C. This chapter includes references to Creech and Paul (2014), who describe a tool called IsoSpike for reduction of double-spike data. A preprint of that paper, currently in press in Geostandards and Geoanalytical Research, is included as Appendix $\mathrm{D}$ of this thesis.

प्य

\section{Abstract}

We report a method for the chemical purification of $\mathrm{Pt}$ from geological materials by ion-exchange chromatography for subsequent $\mathrm{Pt}$ stable isotope analysis by multiple-collector inductively coupled plasma mass spectrometry (MCICPMS) using a ${ }^{196} \mathrm{Pt}-{ }^{198} \mathrm{Pt}$ double-spike to correct for instrumental mass bias. Double-spiking of samples was carried out prior to digestion and chem-

* Author list: John Creech, Joel Baker, Monica Handler and Martin Bizzarro

$\ddagger$ DOI: $10.1016 /$ j.chemgeo.2013.11.009 
ical separation to correct for any mass-dependent fractionation that may occur due to incomplete recovery of Pt. Samples were digested using a NiS fire assay method, which pre-concentrates Pt into a metallic bead that is readily dissolved in acid in preparation for anion-exchange chemistry. $\mathrm{Pt}$ was recovered from anion-exchange resin in concentrated $\mathrm{HNO}_{3}$ acid after elution of matrix elements, including the other platinum group elements (PGE), in dilute $\mathrm{HCl}$ and $\mathrm{HNO}_{3}$ acids. The separation method has been calibrated using a precious metal standard solution doped with a range of synthetic matrices and results in Pt yields $\geq 90 \%$ with purity $\geq 95 \%$. Using this chemical separation technique, we have separated Pt from 11 international geological standard reference materials comprising PGE ores, mantle rocks, igneous rocks and one sample from the Cretaceous-Paleogene boundary layer. Pt concentrations in these samples range from ca. $5 \mathrm{ng} \mathrm{g}^{-1}$ to $4 \mathrm{\mu g} \mathrm{g}^{-1}$. This analytical method has been shown to have an external reproducibility on $\delta^{198} \mathrm{Pt}$ (per mil difference in the ${ }^{198} \mathrm{Pt} /{ }^{194} \mathrm{Pt}$ ratio from the IRMM-010 standard) of $\pm 0.040 \%$ ( $2 \mathrm{sd}$ ) on Pt solution standards (Creech et al., 2013, J. Anal. At. Spectrom. 28, 853-865). The reproducibility in natural samples is evaluated by processing multiple replicates of four standard reference materials, and is conservatively taken to be ca. $\pm 0.088 \%$ o (2 sd). Pt stable isotope data for the full set of reference materials have a range of $\delta^{198} \mathrm{Pt}$ values with offsets of up to $0.4 \%$ from the IRMM-010 standard, which are readily resolved with this technique. These results demonstrate the potential of the $\mathrm{Pt}$ isotope system as a tracer in geochemical systems.

\subsection{Introduction}

Platinum (Pt) has six naturally occurring isotopes $\left({ }^{190} \mathrm{Pt},{ }^{192} \mathrm{Pt},{ }^{194} \mathrm{Pt},{ }^{195} \mathrm{Pt}\right.$, ${ }^{196} \mathrm{Pt}$ and ${ }^{198} \mathrm{Pt}$ ). Like all platinum group elements (PGE; Ru, Rh, Pd, Os, Ir and $\mathrm{Pt}$ ), Pt is highly siderophile and prefers bonds with metal over silicon and oxygen and, as such, is concentrated in the cores of terrestrial planets. The PGE also show some chalcophile behaviour, and in the conditions found in the 
crust and mantle are strongly partitioned into sulphide minerals. Platinum can exist in a range of oxidation states, but in the Earth principally occurs as $\mathrm{Pt}^{0}, \mathrm{Pt}^{2+}$ and $\mathrm{Pt}^{4+}$ Cotton and Wilkinson, 1966). The relatively large mass difference $(2 \%)$ between the abundant heavy and light isotopes of $\mathrm{Pt}$, coupled with its variable redox states in the Earth's core, mantle and surficial environments, and the large differences in Pt abundances that characterise Earth's major geochemical reservoirs suggests that Pt may exhibit significant stable isotope variations. However, to-date there have been no published studies of Pt stable isotope variations in natural terrestrial samples.

We have developed an improved anion-exchange method for chemical separation of Pt from geological materials. Using this method, Pt has been separated from a range of international geological standard reference materials, and their Pt stable isotopic compositions were then determined using a recently developed method for precise Pt stable isotope measurement by double-spike (DS) multiple collector inductively coupled plasma mass spectrometry (MC-ICPMS) (Creech et al., 2013). These results reveal significant differences in Pt stable isotopic composition amongst this suite of samples and provide reference Pt stable isotope data for standards for use in future applications of this technique.

\subsection{Samples}

A suite of 11 international geological standard reference materials were selected based on availability and published Pt contents. The Pt abundances of these samples span practically the entire range found on Earth. A short description of each of the analysed materials is given here and summarised in Table 3.1 .

The standard reference materials are considered in groups as follows:

(a) Pt ore samples: SARM-76 is from the Merensky Reef Ore of the Bushveld Igneous Complex in South Africa, which contains most of the world's known reserves of PGE. The PGE are hosted in extensive sulph- 


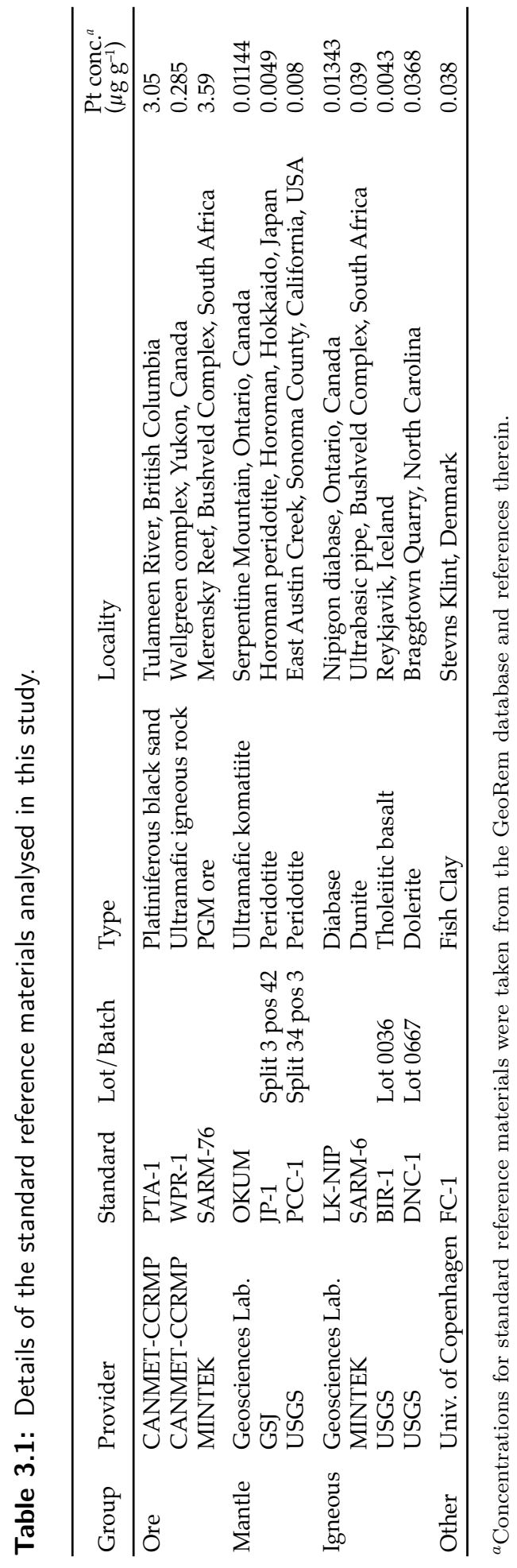


ide layers within the unit, which have an emplacement age of ca. 2.06 Ga (Walraven et al., 1990). SARM-76 is distributed by MINTEK. PTA-1 is a platiniferous black sand from the Tulameen River area of British Columbia, Canada, which is described as being essentially a magnetite concentrate, containing an abundant Pt-Fe alloy (Faye and Sutarno, 1976). The Tulameen ultramafic-gabbro complex has an emplacement age of ca. $186 \mathrm{Ma}$ (Findlay, 1969). The PTA-1 reference material is distributed by the CANMET Canadian Certified Reference Materials Project (CCRMP). WPR-1 is an altered ultramafic igneous rock from the Wellgreen Complex, Yukon, Canada, which is also distributed by CANMET-CCRMP. The Wellgreen Complex is part of the greater Quill Creek ultramafic complex, which has an emplacement age of $232 \mathrm{Ma}$ (Marcantonio et al., 1994). Pt in WPR-1 is thought to be hosted in base metal sulphides (Meisel and Moser, 2004, Barkov et al., 2002), platinum group minerals (Barkov et al., 2002) and tellurides (CANMET-CCRMP reference certificate). The three Pt ore standards have Pt concentrations ranging from 285 to $3590 \mathrm{ng} \mathrm{g}^{-1}$ (Table 3.1).

(b) Mantle-derived samples: PCC-1 is a peridotite standard from California, USA, distributed by the US Geological Survey (USGS), although supplies of this material are now exhausted. JP-1 is a peridotite from the Horoman peridotite, Hokkaido Island, Japan. The petrogenesis of the Horoman peridotite is complicated, but it is considered to have been emplaced ca. $23 \mathrm{Ma}$ (Takazawa et al., 1999) and to represent former suboceanic lithosphere (Takazawa et al., 2000, 1996, 1992). JP-1 is distributed by the Geological Society of Japan (GSJ). OKUM is an ultramafic komatiite distributed by Geosciences Laboratories, that was sampled from Serpentine Mountain, Ontario, Canada. This area is located at the western end of the Abitibi Greenstone Belt, and was emplaced into the crust ca. 2.7 Ga (Houlé et al. 2009). PGE in these rocks are found as Ni-Cu-PGE minerals Houlé et al. 2009). Although OKUM is technically an igneous sample, komatiites are considered to be large degree partial melts of the underlying mantle (e.g. Nisbet and Walker, 1982) and their compositions are expected to reflect the 
mantle source. Platinum concentrations in the mantle-derived samples range from ca. 5-12 $\mathrm{ng} \mathrm{g}^{-1}$ (Table 3.1.

c) Igneous samples: SARM-6 is a dunite from an ultrabasic pipe in the Bushveld Complex, South Africa provided by MINTEK. As with SARM-76 described above, this unit was emplaced ca. 2.06 Ga, and PGE are hosted in sulphide minerals. LK-NIP is a dolerite from the Nipigon Diabase, Ontario, Canada distributed by Geosciences Laboratories. The Nipigon diabase sills were intruded into the crust ca. $1100 \mathrm{Ma}$ (Hart and MacDonald, 2007; Heaman et al. 2007). DNC-1 is a dolerite from North Carolina, USA, distributed by the USGS. BIR-1 is a tholeiitic basalt from a lava flow near Reykjavik, Iceland, distributed by the USGS. These igneous samples have Pt concentrations ranging from ca. $4 \mathrm{ng} \mathrm{g}^{-1}$ in BIR-1 to $39 \mathrm{ng} \mathrm{g}^{-1}$ in SARM-6 (Table 3.1 .

d) Other samples: FC-1 was collected from the Fish Clay at Stevns Klint in Denmark. FC-1 was sampled from a sedimentary layer that is stratigraphically located at the Cretaceous-Paleogene boundary and has elevated levels of Ir, due to the putative meteorite impact at that time (Alvarez et al., 1980). The FC-1 standard is distributed by the University of Copenhagen, and has a Pt concentration of $38 \mathrm{ng} \mathrm{g}^{-1}$ (Table 3.1).

\subsection{Materials and methods}

\subsubsection{Reagents, standard solutions and spikes}

Analytical reagent grade nitric $\left(\mathrm{HNO}_{3}\right)$ and hydrochloric $(\mathrm{HCl})$ acids were purified by sub-boiling distillation in quartz or teflon stills, respectively, prior to use in anion-exchange chemistry. Acid used in the digestion steps (including aqua regia) and all solutions for MC-ICPMS analysis were prepared from Optima TM grade $\mathrm{HNO}_{3}$ and $\mathrm{HCl}$ acids, which were used as supplied without further purification. Acid dilutions and all water rinses were performed with ultra-pure (>18 M $\Omega . c m)$ water. 
In the initial stages of technique development, a mixed 'precious metals' standard from Alfa Aesar supplier (SpecPure plasma standard, $100 \mu \mathrm{g} \mathrm{mL} \mathrm{m}^{-1}$ in $20 \% \mathrm{HCl}$, Lot \# 012912A) was used, containing Ru, Rh, Pd, Re, Os, Ir, $\mathrm{Pt}$ and $\mathrm{Au}$. For performing column calibrations, several synthetic rock mixtures were prepared in order to have compositions approximating those of a peridotite, chondrite, and iron meteorite (Table 3.3) by mixing single-element standard solutions and the precious metal standard solution at the appropriate concentration levels. Mixtures containing all of the elements present in the synthetic rock mixtures were also prepared from standard solutions at concentrations of $1 \mathrm{\mu g} \mathrm{mL} \mathrm{m}^{-1}$ and $1 \mathrm{ng} \mathrm{mL}^{-1}$ and used as a bracketing standards in ICP-MS measurements of ion-exchange fractions for the construction of elemental elution profiles.

The Pt isotope standard IRMM-010 was purchased from the Institute for Reference Materials and Measurements, Belgium. The supplied Pt wire segments were carefully weighed and then cleaned prior to digestion by sequentially rinsing in ultra-pure water, analytical grade methanol and dilute (5\%) $\mathrm{HNO}_{3}$. The cleaned IRMM-010 was then digested in aqua regia $\left(\mathrm{HCl}: \mathrm{HNO}_{3}\right.$ prepared in a volume ratio of 3:1) in a Savillex teflon beaker. The IRMM010 solution was then evaporated, converted to chloride in concentrated $\mathrm{HCl}$, evaporated again, and finally redissolved in $20 \% \mathrm{HCl}$. From this, a $60 \mathrm{ng} \mathrm{mL} \mathrm{m}^{-1}$ solution of IRMM-010 in $0.5 \mathrm{M} \mathrm{HCl}$ was prepared and double-spiked for use as a bracketing standard for MC-ICPMS isotopic measurements (Creech et al. 2013).

The Pt DS used for the isotopic analyses was prepared using two single isotope spikes ( ${ }^{196} \mathrm{Pt}$ and $\left.{ }^{198} \mathrm{Pt}\right)$ obtained from the Oak Ridge National Laboratory, USA. Full details of the design and calibration of the DS are given in Creech et al. (2013).

\subsubsection{Laboratory environment}

Sample digestions were carried out in an ultra-clean laboratory at Victoria University of Wellington. All Pt separation work was conducted in class 10 
laminar flow hoods situated in a class 100 clean laboratory.

Savillex ${ }^{\mathrm{TM}}$ teflon beakers were used for all sample digestions and collection of eluents from chemistry. Beakers were cleaned thoroughly between uses by the following process: pre-cleaning by refluxing with aqua regia overnight on a hotplate at $120^{\circ} \mathrm{C}$; batch cleaning in $6 \mathrm{M} \mathrm{HCl}$ (AR grade) in a large glass beaker overnight on a hotplate at $50{ }^{\circ} \mathrm{C}$; batch cleaning in $7 \mathrm{M} \mathrm{HNO}_{3}(\mathrm{AR}$ grade) in a large glass beaker overnight on a hotplate at $50^{\circ} \mathrm{C}$; two periods of overnight refluxing with aqua regia, prepared from Optima ${ }^{\mathrm{TM}}$ grade $\mathrm{HNO}_{3}$ and $\mathrm{HCl}$ acids, on a hotplate at $120^{\circ} \mathrm{C}$. Beakers were thoroughly rinsed with ultra-pure water between each of these steps.

Crushing of samples or NiS beads was performed using an agate mortar and pestle, which was cleaned thoroughly between uses by rinsing with methanol and ultra-pure water and by grinding pure quartz grains.

\subsubsection{Sample digestion}

Digestion of the standards was carried out using the NiS fire assay technique. This method is commonly used in PGE work, and involves melting the sample in the presence of $\mathrm{Ni}$ and $\mathrm{S}$, which form a metallic NiS bead into which the PGE are sequestered. The NiS bead can then be physically removed from the silicate slag and readily digested in acid. By eliminating most of the rock matrix elements, this approach has the advantage of pre-concentrating the PGE, but can have relatively high blanks when compared with acid digestion techniques (Rehkämper and Halliday, 1997). Most NiS fire assay procedures use relatively large amounts of sample, which has been found to reduce the influence of 'nugget' effects and improve the external reproducibility of PGE concentration measurements. A number of procedures for NiS fire assay have been published, with subtle differences in flux amounts and fusion time (e.g. Rehkämper and Halliday, 1997; Ravizza and Pyle, 1997; Gros et al., 2002, ?; Savard et al., 2010). The procedure used here largely follows ?, although it includes the addition of silica as has been adopted in some other studies (e.g. Gros et al., 2002). 
Powdered material of the terrestrial standards was weighed directly into porcelain crucibles (50 mL, medium wall, Lomb Scientific). The NiS fire assay charges typically comprised $15 \mathrm{~g}$ of sample. When less sample was used due to high Pt concentrations or limited availability of material, the sample weight was made up to $15 \mathrm{~g}$ using pure silica. Procedural blanks for the NiS fire assay technique were assessed by digesting $15 \mathrm{~g}$ of pure silica with no sample.

The samples in the porcelain crucibles were then thoroughly mixed with $10 \mathrm{~g}$ of sodium carbonate, $20 \mathrm{~g}$ of sodium tetraborate, $1 \mathrm{~g}$ of nickel powder and $0.75 \mathrm{~g}$ of sulphur. For samples that were spiked prior to NiS digestion, $\mathrm{Pt}$ DS was added to the mixture in the crucible from a solution of the ${ }^{196} \mathrm{Pt}-{ }^{198} \mathrm{Pt}$ DS in the appropriate volume for a ratio of $55 \% \mathrm{Pt}$ from DS and $45 \%$ natural $\mathrm{Pt}$, and allowed to dry. Crucibles were then placed in a furnace and fused at $950^{\circ} \mathrm{C}$ for $30 \mathrm{~min}$, and at $1050^{\circ} \mathrm{C}$ for a further $30 \mathrm{~min}(?)$. The crucibles were removed from the furnace and left to cool at room temperature. Once cooled, the crucibles were physically broken and NiS beads were carefully removed from the silicate slag. The NiS beads were then weighed and crushed into smaller fragments in an agate mortar and then weighed again before being transferred to Savillex teflon beakers for acid dissolution.

The NiS beads were dissolved in ca. $30 \mathrm{~mL}$ of concentrated $\mathrm{HCl}$ on a hotplate at $120^{\circ} \mathrm{C}$ with lids off, and then left to evaporate. Once dry, another ca. $30 \mathrm{~mL}$ of concentrated $\mathrm{HCl}$ was added to the samples, and the beakers were again placed on a hotplate at $120^{\circ} \mathrm{C}$ with their lids off for $2-3 \mathrm{~h}$. The beakers were removed from the hotplate, and a volume of concentrated $\mathrm{HNO}_{3}$ approximately one-third of that of the remaining $\mathrm{HCl}$ volume was added to produce aqua regia. The beakers were then closed and placed on a hotplate at $120^{\circ} \mathrm{C}$ overnight, following which the samples were typically fully in solution.

The samples were then evaporated to dryness, taken up in ca. 5-10 mL of concentrated $\mathrm{HCl}$ to convert them to chloride form, evaporated to dryness again, and finally taken up in $10 \mathrm{~mL}$ of $0.5 \mathrm{M} \mathrm{HCl}$ in preparation for anion- 
exchange chemistry.

\subsubsection{Anion-exchange chemistry}

Chemical separation of the element of interest is desirable in order to increase analytical sensitivity and to reduce potential isobaric or molecular interferences or matrix effects during isotope analysis by MC-ICPMS. Ionexchange chromatographic techniques have been widely employed for this purpose. Methods for the separation of PGEs from rocks have previously been developed (e.g., Rehkämper and Halliday, 1997; Pearson and Woodland, 2000). However, the focus of these methods has generally been the recovery of all the PGE for the determination of their concentrations. In this study, we developed a technique for the recovery and purification solely of Pt at a level suitable for precise determination of isotopic compositions by MC-ICPMS.

Prior to loading in columns, Bio-Rad® AG1-X8 anion exchange resin (100-200 mesh, chloride form) was sequentially cleaned by shaking in ultrapure water, $6 \mathrm{M} \mathrm{HCl}$, ultra-pure water, $4 \mathrm{M} \mathrm{HNO}_{3}$ and then ultra-pure water, with the liquid decanted off between each step (Rehkämper and Halliday, 1997). All ion-exchange separations were performed using Eichrom polypropylene columns with a $2 \mathrm{~mL}$ resin capacity and a $20 \mathrm{~mL}$ reservoir. Approximately $1 \mathrm{~mL}$ of fresh BioRad® AG1-X8 anion exchange resin was loaded as a slurry into the columns and allowed to gravity settle. The resin bed was cleaned prior to use with the following sequence of reagents: $20 \mathrm{~mL}$ of $0.8 \mathrm{M} \mathrm{HNO}_{3}, 10 \mathrm{~mL}$ of $11 \mathrm{M} \mathrm{HCl}, 25 \mathrm{~mL}$ of $13.5 \mathrm{M} \mathrm{HNO}_{3}$ and $40 \mathrm{~mL}$ of 6 $\mathrm{M} \mathrm{HCl}$ (for reconversion to chloride form) (Rehkämper and Halliday, 1997).

Immediately prior to loading, columns were equilibrated using $40 \mathrm{~mL}$ of $0.5 \mathrm{M} \mathrm{HCl}$. The elution sequence used is shown in Fig. 3.1. Samples were loaded onto the columns in $10 \mathrm{~mL}$ of $0.5 \mathrm{M} \mathrm{HCl}$. Loading was followed by a $40 \mathrm{~mL}$ rinse with $0.5 \mathrm{M} \mathrm{HCl}$, during which virtually all of the major cationic constituents of the sample and some of the weakly retained PGE ( Rh, Ru and Ir) were eluted. This was followed by elution of $20 \mathrm{~mL}$ of 1 


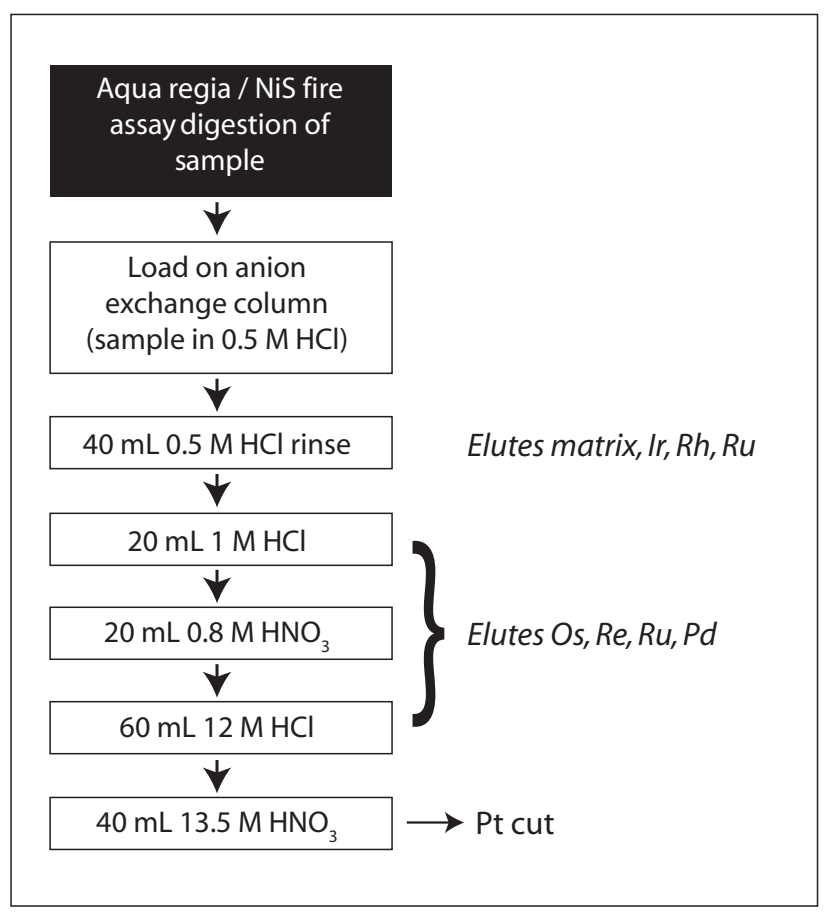

Figure 3.1: Flow chart illustrating the Pt separation scheme

$\mathrm{M} \mathrm{HCl}$, which elutes any remaining weakly retained PGE. Following these $\mathrm{HCl}$ elutions, $20 \mathrm{~mL}$ of $0.8 \mathrm{M} \mathrm{HNO}_{3}$ was added to elute transition metals such as Zn (Rehkämper and Halliday, 1997; Pearson and Woodland, 2000). This was followed by elution of $60 \mathrm{~mL}$ of $11 \mathrm{M} \mathrm{HCl}$, which elutes Re, Pd and any remaining $\mathrm{Ru}$ from the resin. The Pt fraction was finally eluted with 40 $\mathrm{mL}$ of $13.5 \mathrm{M} \mathrm{HNO}_{3}$. Pt cuts were collected in Savillex teflon beakers and evaporated to dryness on a hotplate at $120^{\circ} \mathrm{C}$. During elution tests, cuts from every step were collected in $20 \mathrm{~mL}$ fractions, apart from the $0.5 \mathrm{M} \mathrm{HCl}$ wash which was collected in one $40 \mathrm{~mL}$ fraction.

To monitor procedural blanks, a blank sample solution was processed through anion-exchange chemistry with each batch of samples. 


\subsubsection{Column calibration by ICP-MS}

Prior to analysis, dried fractions from the column calibration experiments were converted to chloride form by taking them up in concentrated $\mathrm{HCl}$ and evaporating before being redissolved in $5 \mathrm{~mL}$ of $0.5 \mathrm{M} \mathrm{HCl}$ and left on a hotplate for 2-3 h before analysis by ICP-MS. An Agilent 7500CS quadrupole ICP-MS with a standard cross-flow nebuliser was used to measure the column fractions. The following masses were monitored for column calibration experiments with the precious metal standard: ${ }^{99} \mathrm{Ru},{ }^{103} \mathrm{Rh},{ }^{105} \mathrm{Pd}$, ${ }^{185} \mathrm{Re},{ }^{189} \mathrm{Os},{ }^{191} \mathrm{Ir}$ and ${ }^{195} \mathrm{Pt}$. Analyses of the column calibration cuts were bracketed with analyses of a gravimetrically prepared solution of the same precious metal standard with a concentration of $1 \mathrm{ng} \mathrm{mL}^{-1}$.

For column calibration experiments with synthetic rock matrices, the following masses were monitored: ${ }^{23} \mathrm{Na},{ }^{25} \mathrm{Mg},{ }^{27} \mathrm{Al},{ }^{29} \mathrm{Si},{ }^{33} \mathrm{~S},{ }^{43} \mathrm{Ca},{ }^{47} \mathrm{Ti},{ }^{52} \mathrm{Cr}$, ${ }^{55} \mathrm{Mn},{ }^{57} \mathrm{Fe},{ }^{59} \mathrm{Co},{ }^{60} \mathrm{Ni},{ }^{63} \mathrm{Cu},{ }^{66} \mathrm{Zn},{ }^{71} \mathrm{Ga},{ }^{72} \mathrm{Ge},{ }^{75} \mathrm{As},{ }^{95} \mathrm{Mo},{ }^{99} \mathrm{Ru},{ }^{103} \mathrm{Rh}$, ${ }^{105} \mathrm{Pd},{ }^{118} \mathrm{Sn},{ }^{121} \mathrm{Sb},{ }^{182} \mathrm{~W},{ }^{185} \mathrm{Re},{ }^{189} \mathrm{Os},{ }^{191} \mathrm{Ir},{ }^{195} \mathrm{Pt}$ and ${ }^{197} \mathrm{Au}$. Analyses of the column calibration cuts were bracketed with standard solutions containing all of these elements $\left(1 \mu \mathrm{g} \mathrm{mL} L^{-1}\right.$ and $\left.1 \mathrm{ng} \mathrm{mL}^{-1}\right)$ that were gravimetrically prepared from single element standards. Semi-quantitative concentrations of the relevant elements were calculated for each fraction relative to the measured signal intensities for the standard. Elution curves constructed with these data are estimated to be accurate to $\pm 5 \%$.

\subsubsection{Platinum stable isotope analysis by double-spike MC-ICPMS}

A ${ }^{196} \mathrm{Pt}-{ }^{198} \mathrm{Pt}$ DS was used to correct for instrumental mass fractionation during MC-ICPMS analysis. The development of the Pt DS and mass spectrometry protocols are described in detail by Creech et al. (2013).

Samples for isotopic analysis were either spiked before sample digestion or prior to ion-exchange chemistry. Samples that were spiked prior to ionexchange chemistry were refluxed in aqua regia on a hotplate at $120^{\circ} \mathrm{C}$ 
overnight to ensure complete sample-spike equilibration. These sample-spike mixtures were subsequently evaporated to dryness, reconverted to chloride form by evaporating concentrated $\mathrm{HCl}$, and finally redissolved in $10 \mathrm{~mL}$ of $0.5 \mathrm{M} \mathrm{HCl}$ for loading onto columns.

In order to destroy any potential organic interferences arising from resin that may have been stripped from the resin bed by the large volume of concentrated $\mathrm{HNO}_{3}$ that was used to elute Pt, the Pt cuts were treated after chemistry by the following steps. Pt cuts were evaporated to dryness, refluxed in aqua regia on a hotplate overnight, evaporated to dryness again, and then refluxed overnight in a mixture of $\mathrm{HNO}_{3}-\mathrm{H}_{2} \mathrm{O}_{2}$. The Pt cuts were then evaporated to dryness, reconverted to chloride form by evaporating concentrated $\mathrm{HCl}$, and finally redissolved in an appropriate volume of $0.5 \mathrm{M}$ $\mathrm{HCl}$ resulting in a $\mathrm{Pt}$ solution concentration of approximately $60 \mathrm{ng} \mathrm{mL}^{-1}$. Samples were refluxed on a hotplate for at least 2 to $3 \mathrm{~h}$ prior to analysis.

Pt stable isotope measurements were carried out using a Nu Plasma MCICPMS at Victoria University of Wellington, New Zealand. The Nu Plasma MC- ICPMS was operated in low-resolution mode, with a mass resolution of 1840 (M/ $\Delta \mathrm{M}$; peak edge width from 5-95\% full peak height). A Nu Instruments DSN-100 desolvation nebuliser system with an ESI PFA micro-flow nebuliser was used for $\mathrm{Pt}$ solution introduction to the mass spectrometer.

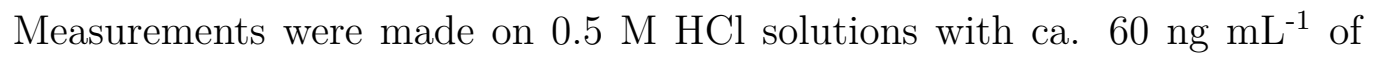
natural Pt. The sample uptake rate was ca. $80 \mathrm{~mL} \mathrm{~min}^{-1}$, and the sensitivity of the instrument was typically ca. $280 \mathrm{~V} \mathrm{ppm}^{-1}$ total Pt under these conditions. Each analysis comprised $100 \times 8$ s integrations, corresponding to 13.3 min of data acquisition, and consumed ca. $80 \mathrm{ng}$ of natural Pt. Baselines were obtained by measuring on-peak for $100 \times 8$ s integrations prior to each analysis. A washout time of ca. 30 min was applied between analyses and baseline measurements. Using this approach, background levels were typically $<1 \mathrm{mV}$ on all $\mathrm{Pt}$ isotopes.

Data reduction was performed off-line using the freely available data reduction software Iolite, which runs within Igor Pro (Paton et al., 2011). The 
DS calculations were performed algebraically based on the equations presented in Rudge et al. (2009) using a new procedure incorporated within Iolite (Creech and Paul, 2014). We report Pt stable isotope results in terms of $\delta^{198} \mathrm{Pt}$, representing the per mil deviation of the ${ }^{198} \mathrm{Pt} /{ }^{194} \mathrm{Pt}$ ratio in the sample from the same ratio for the IRMM-010 Pt isotope standard. This technique has been shown to have an external reproducibility on $\delta^{198} \mathrm{Pt}$ measurements of Pt solution standards of $\pm 0.04 \%$ o (i.e. $\pm 0.01 \%$ o amu ${ }^{-1}$; Creech et al., 2013).

\subsection{Results and Discussion}

\subsubsection{Chemical separation and recovery of $\mathrm{Pt}$}

Results of elution tests for the chemical separation of Pt are shown in Fig. 3.2 Despite undertaking these column calibration experiments on a wide range of synthetically prepared materials, there is little difference in the elution profiles of the matrix elements and PGE. In all of the elution tests, almost all of the matrix elements are entirely eluted from the column prior to $\mathrm{Pt}$ collection, passing through the column in the loading acid. The exceptions to this are $\mathrm{Zn}$, which is eluted in $0.8 \mathrm{M} \mathrm{HNO}_{3}$, minor amounts of Si and Sn that were occasionally found in other cuts, and Au which was found to also be completely recovered in the $13.5 \mathrm{M} \mathrm{HNO}_{3}$ cut.

$\mathrm{Rh}$ is only very weakly retained on the anion resin, and is almost entirely eluted in the sample loading solution, with any remaining Rh being eluted in the $0.5 \mathrm{M} \mathrm{HCl}$ wash. $\mathrm{Ru}$ and Ir are also relatively weakly retained on the

column. Previous studies have used oxidants (e.g., bromine water, chlorine gas) to convert Ir to $\operatorname{Ir}(\mathrm{IV})$, which is more strongly retained on the resin, and can be subsequently eluted with the addition of a reducing agent. However, given that we did not use such a step, Ir is only weakly retained and is largely eluted during the $0.5 \mathrm{M} \mathrm{HCl}$ rinse after loading and, hence, is presumably mostly in the reduced state (i.e., Ir(III)). In some cases, traces of Ir were 


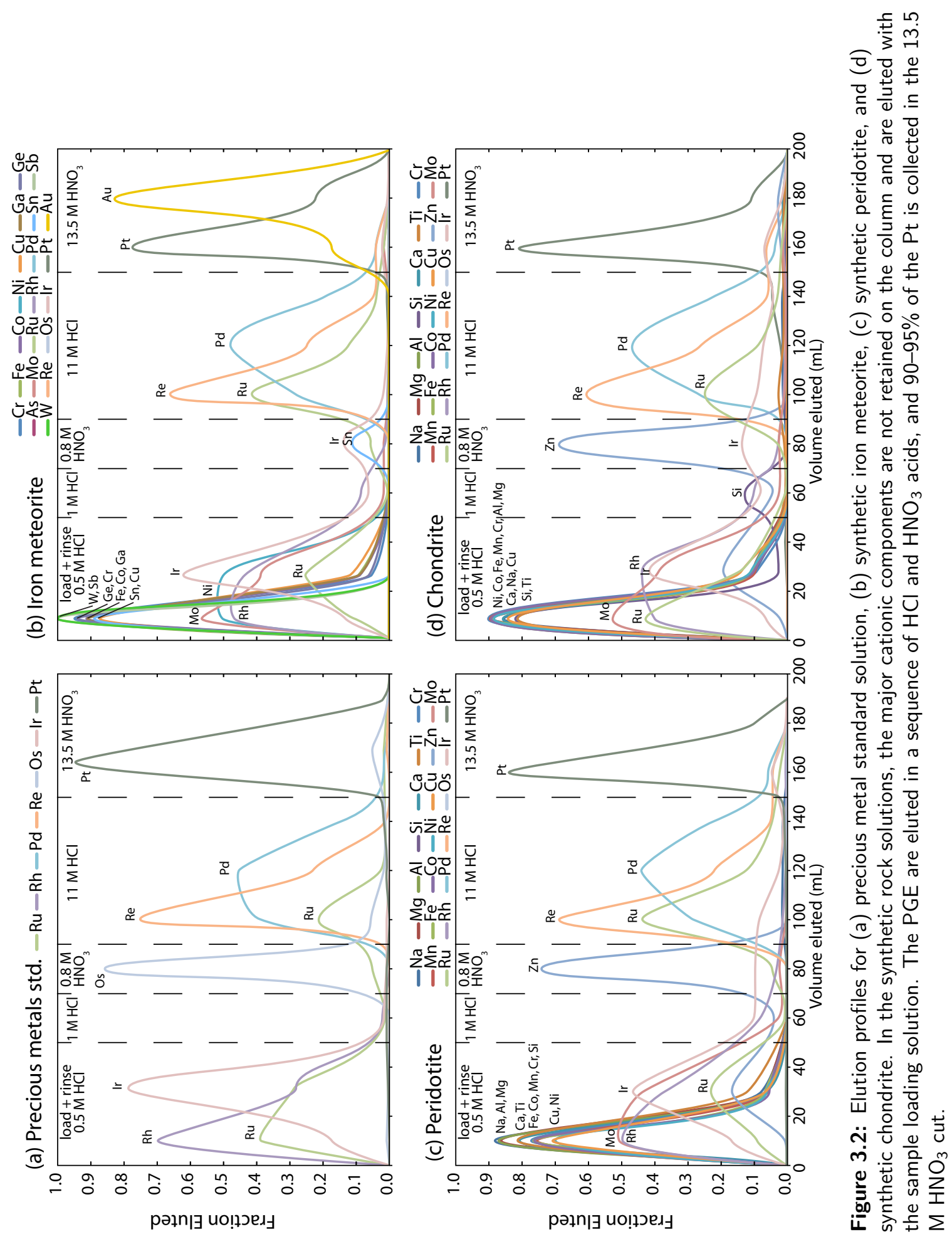


found to be present in the $0.8 \mathrm{M} \mathrm{HNO}_{3}$ or $11 \mathrm{M} \mathrm{HCl}$ cuts and, thus, some Ir was likely present as $\operatorname{Ir}(\mathrm{IV})$. The same is also probably the case for $\mathrm{Ru}$, which is mostly eluted during the post-loading wash, but also shows a second peak in the $11 \mathrm{M} \mathrm{HCl}$ cuts. During elution tests with the precious metal solution, $80-90 \%$ of the Os was eluted in $0.8 \mathrm{M} \mathrm{HNO}_{3}$. However, in all of the elution tests with a synthetic rock matrix, virtually no Os was present. Given that multiple evaporations of the solutions were conducted in the preparation of these samples and the highly volatile nature of Os, it is likely that Os was lost during those steps. Re was largely eluted in the first $20 \mathrm{~mL}$ of the $11 \mathrm{M}$ $\mathrm{HCl}$ cut, with the remainder being eluted in the next $20 \mathrm{~mL}$ of $11 \mathrm{M} \mathrm{HCl}$. $\mathrm{Pd}$ is less easily eluted than Re in $11 \mathrm{M} \mathrm{HCl}$, with ca. $30 \%$ of the Pd being eluted in the first and last $20 \mathrm{~mL}$ volumes of $11 \mathrm{M} \mathrm{HCl}$, and ca. $40 \%$ in the middle $20 \mathrm{~mL}$ volume of $11 \mathrm{M} \mathrm{HCl}$.

$\mathrm{Pt}$ is finally eluted in $40 \mathrm{~mL}$ of $13.5 \mathrm{M} \mathrm{HNO}_{3}$. At least $80 \%$ of $\mathrm{Pt}$ is eluted in the first $20 \mathrm{~mL}$ of $13.5 \mathrm{M} \mathrm{HNO}_{3}$, but a further $20 \mathrm{~mL}$ of $13.5 \mathrm{M} \mathrm{HNO}_{3}$ was eluted to ensure (near) complete recovery of Pt. At least 90-95\% of Pt is recovered in the combined $40 \mathrm{~mL}$ of $13.5 \mathrm{M} \mathrm{HNO}_{3}$. This level of recovery was deemed adequate given that isotopic samples were double-spiked prior to anion-exchange chemistry and, thus, incomplete recovery should not affect the measured isotopic compositions of samples. This was tested by processing a sample of IRMM-010 through the entire sample preparation procedure, whereby a NiS blank charge was prepared as described above and a doublespiked aliquot of IRMM-010 was added to it. The results of this test are discussed below. Minor amounts of the other PGE were also recovered in the Pt cut, but these were all $<5 \%$ of the Pt concentration, and this level has been previously shown to be sufficient for accurate stable isotope determinations of Pt (Creech et al. 2013).

As a large fraction of $\mathrm{Au}$ was collected with $\mathrm{Pt}$, and the effects of this on DS MC-ICPMS isotopic analysis of Pt were only explored up to $10 \%$ of the Pt concentration by Creech et al. (2013), an analytical test was carried out by doping IRMM-010 with $\mathrm{Au}$ at $50 \%$ of the $\mathrm{Pt}$ concentration. $\mathrm{Au}$ 
doping at $30 \mathrm{ng} \mathrm{mL} \mathrm{m}^{-1}$ produced a signal of ca. $3 \mathrm{~V}$ on ${ }^{197} \mathrm{Au}$, which is higher than was observed in any samples analysed in this study. Au doped Pt stable isotope analyses had a reproducibility on $\delta^{198} \mathrm{Pt}$ identical to those of undoped IRMM-010 (ca. $\pm 0.042 \%$; $2 \mathrm{sd}$ ) and Pt stable isotope ratios identical to pure IRMM-010 (Table 3.2).

The total procedural blank of the analytical procedure was monitored by processing a blank with each batch of samples through anion-exchange chemistry, both with and without NiS fire assay. Anion-exchange column Pt blanks were typically ca. $\leq 0.1 \mathrm{ng}$. Combined Pt blanks from NiS digestions and anion-exchange chemistry were typically ca. $0.5-2 \mathrm{ng}$. The importance of blanks at these levels for accurate isotopic measurements is discussed below.

\subsubsection{Pt concentrations of standards}

Pt concentrations of the standard reference materials were determined by performing isotope dilution calculations on double-spiked samples, treating ${ }^{198} \mathrm{Pt}$ as the spike isotope and ${ }^{194} \mathrm{Pt}$ as the unspiked isotope (although some ${ }^{194} \mathrm{Pt}$ is also present in the DS). The accuracy of the calculated sample concentrations potentially depends on when spiking was carried out (i.e., prior to or after NiS fire assay digestion) as spiking after digestion will only yield accurate concentrations if the NiS fire assay quantitatively recovers all the Pt. In samples that were double-spiked prior to NiS fire assay, concentrations determined by isotope dilution calculations were between $80-108 \%$ of reference Pt concentrations (Table 3.2). The sample of the komatiite standard OKUM that was double-spiked prior to NiS fire assay had a Pt concentration of 12.4 $\mu \mathrm{g} \mathrm{g}{ }^{-1}$ (reference Pt $=11.44 \mu \mathrm{g} \mathrm{g}^{-1}$ ), compared with $9.0 \mu \mathrm{g} \mathrm{g}^{-1}$ when spiking took place after digestion. This is consistent with Pt concentrations determined from replicate WPR-1 digestions, where double-spiking took place after digestion, for which calculated Pt concentrations vary between ca. 80-100\% of the reference value. 


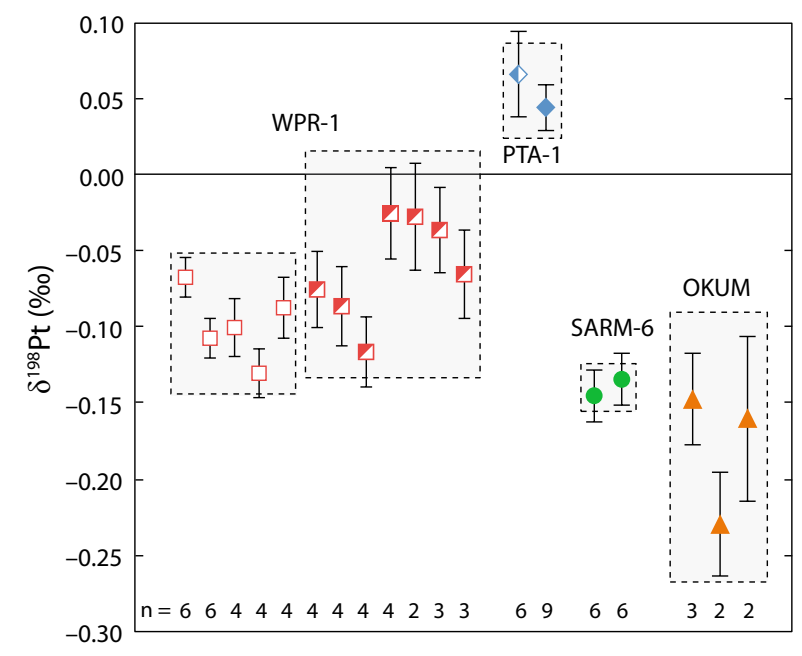

Figure 3.3: Pt stable isotopic measurements of the standard reference materials WPR-1, PTA- 1, SARM-6 and OKUM, for which replicate digestions were processed. Hollow symbols indicate that the sample was double-spiked after NiS digestion and anion-exchange chemistry, half-filled symbols indicate that the sample was double-spiked after NiS digestion but prior to anion-exchange chemistry, and filled symbols indicate that the sample was double-spiked prior to both NiS digestion and anion-exchange chemistry. Data points are error-weighted means of the number of analyses $(n)$ indicated below each data point, with associated combined errors. Dashed lines indicate the 2 sd uncertainty from all of the replicates they enclose.

\subsubsection{Platinum stable isotope results}

\section{Reproducibility of processed standards}

The reproducibility of $\mathrm{Pt}$ stable isotopic measurements on the geological standards was estimated by measuring replicate digestions of several standard reference materials. Seven replicate digestions of WPR-1 were split and processed twice, once with double-spiking prior to anion-exchange chemistry, and once with double-spiking after anion-exchange chemistry. In addition, the three standards OKUM, SARM-6 and PTA-1 were also digested 2-3 times. One digestion of PTA-1 was double-spiked before NiS digestion and the other was double-spiked prior to anion-exchange chemistry. All digestions of OKUM and SARM-6 were double-spiked prior to NiS digestion. Each processed digestion was typically analysed 3-6 times. Pt stable isotope results for the replicate digestions of standard reference materials are shown in Table 


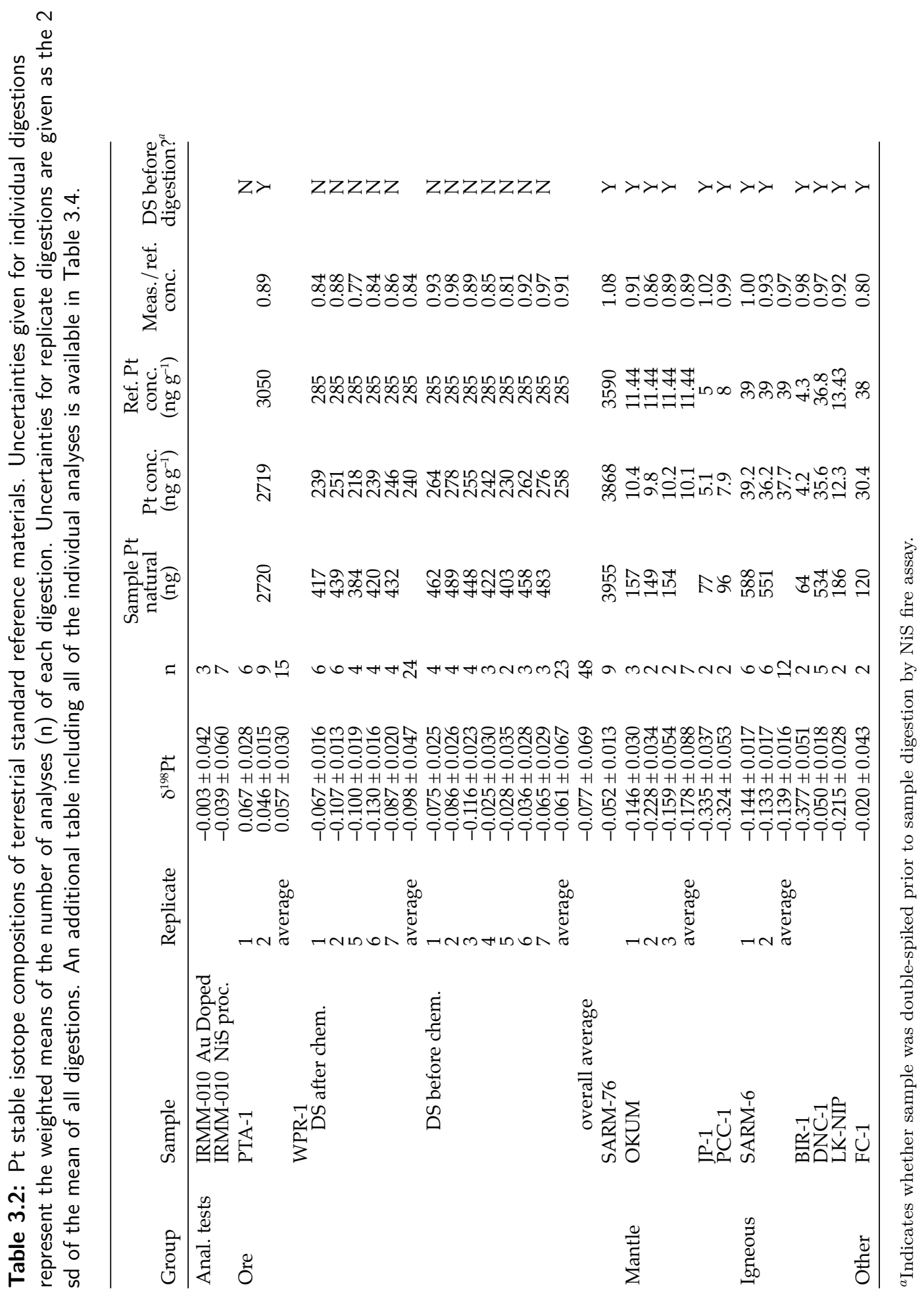


3.2 and Fig. 3.3 .

Repeat digestions of PTA-1 and SARM-6 yielded uncertainties of $\pm 0.030 \%$ and $\pm 0.016 \%$ ( 2 sd of weighted means), respectively. Replicates of OKUM were less reproducible, with an uncertainty of $\pm 0.088 \%$ o ( $2 \mathrm{sd}$ of weighted means). WPR-1 replicates where double-spiking was carried out after anion exchange chemistry yielded a mean $\delta^{198} \mathrm{Pt}=-0.098 \pm 0.047 \%$ o $(2$ sd of weighted means), whereas those spiked prior to anion exchange chemistry yielded a mean $\delta^{198} \mathrm{Pt}=-0.061 \pm 0.067 \%$ o (2 sd of weighted means). These yield a combined average for WPR- 1 of $\delta^{198} \mathrm{Pt}=-0.077 \pm 0.069 \%$ o (2 sd). Additionally, the NiS-processed IRMM-010 sample (described in section 4.1) yielded a mean $\delta^{198} \mathrm{Pt}$ of $-0.039 \pm 0.060 \%$ ( $2 \mathrm{sd}$; Table 3.2. Fig. 3.4. The uncertainty demonstrated by these tests must be taken into account when interpreting data from single digestions of samples. Possible explanations for the poorer reproducibility of measurements of replicate digestions of WPR-1 and OKUM compared with that of Pt solution standards include:

a) Anion exchange chemistry yields. WPR-1 replicates spiked before and after chemistry were spiked with identical amounts of DS. Isotope dilution concentration results for WPR-1 replicates spiked after anion exchange chemistry have ca. $10 \%$ lower apparent Pt concentrations than those spiked prior to chemistry, consistent with elution results shown in Section 3.4.1. The overlap in $\delta^{198} \mathrm{Pt}$ results for replicates spiked before and after anion exchange chemistry suggests that incomplete recovery of $\mathrm{Pt}$ during anion-exchange chemistry does not isotopically fractionate Pt significantly at this level of recovery. Additionally, no correlation is observed between $\delta^{198} \mathrm{Pt}$ and $\mathrm{Pt}$ recovery within WPR-1 replicates spiked after chemistry (Table 3.2. Thus, yields from anion exchange chemistry can not explain the variations observed in the WPR-1 replicates.

b) Incomplete sample-spike equilibration. Incomplete sample-spike equilibration could result in an apparent isotopic fractionation of a sample. Although all of the WPR-1 replicates were subjected to identical preparation that was intended to equilibrate the sample and DS, incomplete and in- 
consistent equilibration could explain some of the variability in the WPR-1 replicates, and potentially explain why the replicates double-spiked before anion-exchange chemistry show poorer reproducibility than those doublespiked after chemistry. However, samples double-spiked prior to digestion are considered to have fully equilibrated.

c) NiS fire assay blanks. Pt blanks from the NiS fire assay technique were found to vary between ca. 0.5-2.0 ng. The influence of blank Pt is difficult to assess without knowing the isotopic composition of the blank, and depends also on the Pt content of the sample. Given that each digestion of WPR-1 involved processing of ca. $4000 \mathrm{ng}$ of $\mathrm{Pt}$ it can be calculated that the stable Pt isotopic composition of the blank would need to have an implausibly high $\delta^{198} \mathrm{Pt}$ of ca. $+10 \%$ and be highly variable either in terms of this blank composition or content to account for the range of $\delta^{198} \mathrm{Pt}$ values exhibited by the replicate digestions of WPR-1. Furthermore, the Pt concentrations obtained for our most Pt depleted samples agree well with published reference values for these standards.

d) Spectral or non-spectral matrix effects from residual organics from anion exchange resin. Element doping tests have shown that minor amounts of the most likely contaminants from incomplete Pt separation should not affect isotopic measurements (Creech et al. 2013). As described above, Pt cuts were treated with aqua regia and $\mathrm{HNO}_{3}-\mathrm{H}_{2} \mathrm{O}_{2}$ to attempt to destroy any organics stripped from the anion exchange resin. While this is considered to be effective, the presence of any residual organics may have variable spectral or non-spectral matrix effects that could explain the variations observed between replicates of WPR-1.

e) True isotopic heterogeneity of the sample. This may be more apparent with OKUM than the other standards due to the lower Pt concentration in that standard. However, due to its lower concentration, each replicate of OKUM was only measured 2-3 times, which also affects the resulting uncertainty. Further work is required to establish the level of Pt isotopic heterogeneity within various types of samples. It is hoped that additional 


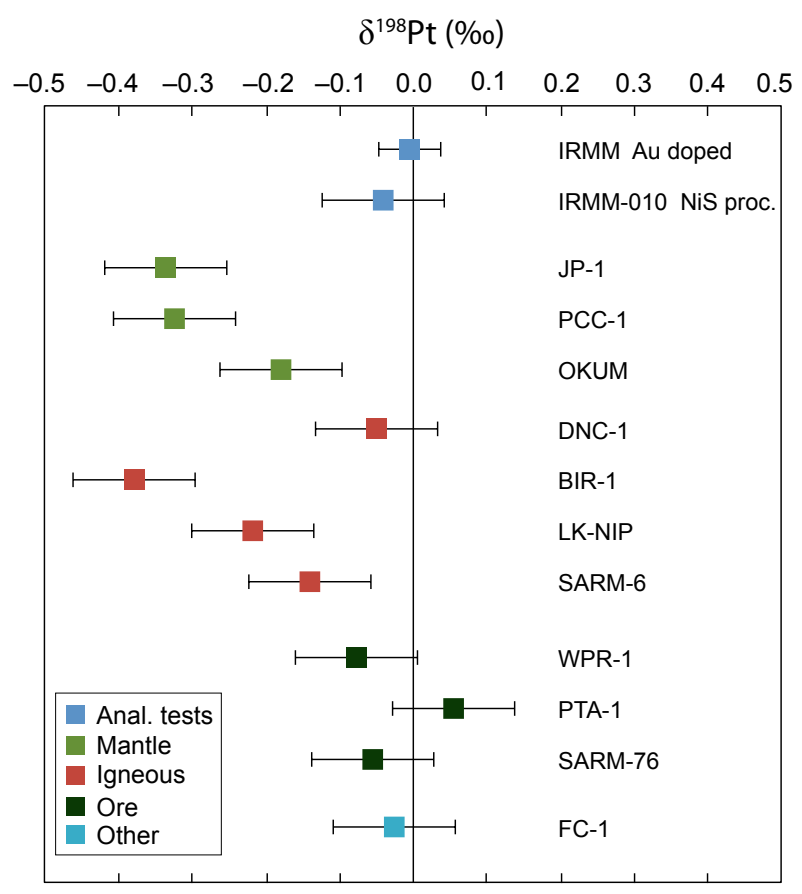

Figure 3.4: Pt stable isotope variations in standard reference materials relative to IRMM-010. Error bars reflect the reproducibility as determined by the 2 sd of replicate digestions of OKUM of $\pm 0.088 \%$ o (Table 3.2).

investigations utilising these methods will be forthcoming, which will help to resolve this question.

The relatively consistent results between replicate digestions of SARM-6 and PTA-1 suggest that reproducibility may be improved where samples are double-spiked prior to digestion (Fig. 3.3). However, for the data for natural samples presented in Fig. 3.4 we have conservatively imposed uncertainties of $\pm 0.088 \%$ o (Table 3.2), as determined from replicate digestions of OKUM.

\section{Pt stable isotope variations in terrestrial rock standards}

The 11 standard reference materials analysed in this study showed Pt stable isotope variations that are readily resolvable by this technique (Fig. 3.4 Table 3.2). The Pt stable isotope composition of Earth's mantle as defined by mantle samples JP-1, PCC-1 and OKUM, is clearly distinct from those of 
the ultramafic ore deposits PTA-1 and SARM-76. The two peridotites (JP-1 and PCC-1) have identical Pt stable isotope compositions within analytical uncertainty $\left(\delta^{198} \mathrm{Pt}=-0.335 \%\right.$ and $-0.324 \%$, respectively $)$.

The ore materials, WPR-1, SARM-76 and PTA-1, have the heaviest Pt isotopic compositions, which are similar to the composition of the IRMM010 standard (i.e. close to zero). The similarity between the ore materials and IRMM-010 may be consistent with the IRMM-010 Pt isotope standard having been ultimately derived from a $\mathrm{Pt}$ ore. The igneous group of reference materials generally have $\mathrm{Pt}$ stable isotopic compositions intermediate between the mantle and ores. Although the ores used in this study may have formed through different processes, the isotopic offset between igneous samples and ores suggests that there may an isotopic fractionation associated with the removal of $\mathrm{Pt}$ from silicate magmas to form $\mathrm{Pt}$ ore deposits. The Icelandic basalt BIR-1, which is a mafic and isotopically depleted product of the Iceland plume, has the most extreme Pt stable isotopic composition of $\delta^{198} \mathrm{Pt}=-0.377 \pm 0.051 \%$. This composition is similar to that of the peridotite samples, which are considered to represent the upper mantle.

DNC-1 has the heaviest Pt stable isotopic composition of the igneous standard reference materials $\left(\delta^{198} \mathrm{Pt}=-0.050 \% 0\right)$. DNC-1 is also characterised by an unusually high Pt concentration (38 $\mathrm{ng} \mathrm{g}^{-1}$; Table 3.1) compared with other igneous samples. The isotopic similarity between DNC-1 and the ore samples coupled with its relative enrichment in Pt potentially indicates that either the source deposit of DNC-1 is associated with sulphide-enriched ultramafic deposits or that the DNC-1 standard was contaminated during crushing and preparation of the standard. Similar contamination during preparation and crushing of geological standard reference materials has been documented for other isotopic systems (e.g., $\mathrm{Pb}$; Baker et al., 2004).

The reproducibility of $\mathrm{Pt}$ stable isotope data documented in the standard reference materials of which multiple digestions were made in this study, particularly SARM-6 and PTA-1, demonstrates that these could be potentially useful as isotopic standards in future applications of $\mathrm{Pt}$ and other highly 
siderophile element stable isotope studies.

\subsection{Conclusions}

We have developed a chemical separation procedure for purification of $\mathrm{Pt}$ from geological and potentially from meteoritic samples for Pt stable isotopic analysis. We have shown:

1. Pt can be separated from various rock matrices with a recovery of 90-95\% and with sufficient purity (>95\%) for accurate Pt stable isotope determinations.

2. While the current reproducibility of processed replicates of terrestrial rock standards (e.g., ca. $\pm 0.088 \%$ o for OKUM) is poorer than for repeat measurements of Pt solution standards (ca. $\pm 0.040 \%$ ), this level of precision appears to be adequate to resolve variations between natural samples.

3. Pt stable isotopic analyses of 11 terrestrial standard reference materials show a readily resolvable range in $\delta^{198} \mathrm{Pt}$, with offsets of up to ca. $0.4 \%$ o for some standards.

These results demonstrate that the Pt stable isotope system holds considerable promise as a tracer in geochemical systems.

\section{Acknowledgements}

The authors thank two anonymous reviewers for their insightful comments and Laurie Reisberg for her editorial efforts, which improved the manuscript. This research was supported by a Royal Society of New Zealand Marsden Grant to MH and JB. JC is funded by a VUW PhD scholarship. The Centre for Star and Planet Formation is funded by the Danish National Research Foundation and the University of Copenhagen's Programme of Excellence. 


\section{Supplementary material}

The published version of this chapter included two supplementary tables, numbered as Table S1 and Table S2. They are included below, and are numbered in the text as Table 3.3 and Table 3.4, respectively. 


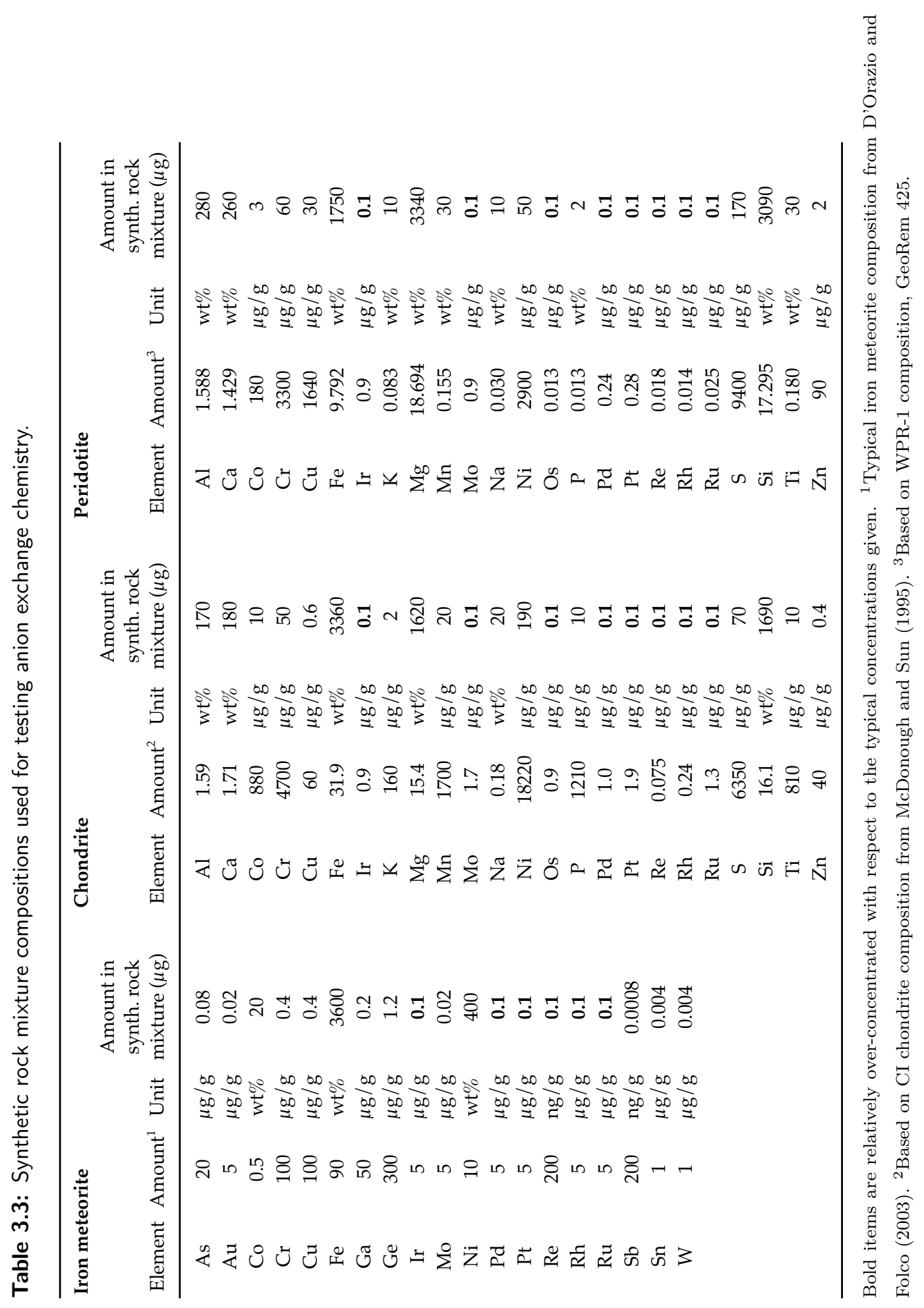


Table 3.4: Pt stable isotope results for individual analyses, with weighted means for each replicate as given in the main text.

\begin{tabular}{|c|c|c|c|c|c|c|c|c|c|c|}
\hline Group & Sample & Replicate \# & $\delta^{198} \mathrm{Pt}$ & $\begin{array}{c}\text { internal } \\
\text { error } \\
(2 \mathrm{se})\end{array}$ & $\begin{array}{c}\mathrm{ng} \mathrm{Pt} \\
\text { (natural) }\end{array}$ & $\begin{array}{l}\text { apparent Pt } \\
\text { conc. (ng/g) }\end{array}$ & $\begin{array}{c}\text { ref. conc } \\
(\mathrm{ng} / \mathrm{g})\end{array}$ & $\begin{array}{c}\text { meas. / } \\
\text { ref. }\end{array}$ & $\begin{array}{l}\text { weighted } \\
\text { mean }\end{array}$ & \pm \\
\hline \multirow{15}{*}{\multicolumn{2}{|c|}{ PTA-1 }} & 1 & 0.037 & 0.066 & & & & & & \\
\hline & & 1 & 0.067 & 0.057 & & & & & & \\
\hline & & 1 & 0.088 & 0.069 & & & & & & \\
\hline & & 1 & 0.120 & 0.120 & & & & & & \\
\hline & & 1 & 0.060 & 0.077 & & & & & & \\
\hline & & 1 & 0.069 & 0.061 & & & & & 0.067 & 0.028 \\
\hline & & 2 & 0.054 & 0.044 & 2721 & 2719 & 3050 & 0.89 & & \\
\hline & & 2 & 0.022 & 0.044 & 2721 & 2719 & 3050 & 0.89 & & \\
\hline & & 2 & -0.001 & 0.045 & 2720 & 2719 & 3050 & 0.89 & & \\
\hline & & 2 & 0.060 & 0.043 & 2720 & 2719 & 3050 & 0.89 & & \\
\hline & & 2 & 0.063 & 0.070 & 2720 & 2719 & 3050 & 0.89 & & \\
\hline & & 2 & 0.079 & 0.043 & 2720 & 2719 & 3050 & 0.89 & & \\
\hline & & 2 & -0.015 & 0.050 & 2721 & 2719 & 3050 & 0.89 & & \\
\hline & & 2 & 0.070 & 0.041 & 2720 & 2719 & 3050 & 0.89 & & \\
\hline & & 2 & 0.064 & 0.041 & 2720 & 2719 & 3050 & 0.89 & 0.046 & 0.015 \\
\hline & \multirow{24}{*}{ WPR-1, DS after chemistry } & 1 & -0.136 & 0.032 & 417 & 239 & 285 & 0.84 & & \\
\hline & & 1 & -0.079 & 0.026 & 417 & 239 & 285 & 0.84 & & \\
\hline & & 1 & -0.043 & 0.038 & 417 & 239 & 285 & 0.84 & & \\
\hline & & 1 & -0.040 & 0.038 & 417 & 239 & 285 & 0.84 & & \\
\hline & & 1 & -0.046 & 0.031 & 417 & 239 & 285 & 0.84 & & \\
\hline & & 1 & -0.041 & 0.032 & 417 & 239 & 285 & 0.84 & -0.067 & 0.016 \\
\hline & & 2 & -0.153 & 0.029 & 439 & 251 & 285 & 0.88 & & \\
\hline & & 2 & -0.147 & 0.031 & 439 & 251 & 285 & 0.88 & & \\
\hline & & 2 & -0.099 & 0.031 & 439 & 251 & 285 & 0.88 & & \\
\hline & & 2 & -0.096 & 0.036 & 439 & 251 & 285 & 0.88 & & \\
\hline & & 2 & -0.059 & 0.036 & 439 & 251 & 285 & 0.88 & & \\
\hline & & 2 & -0.067 & 0.030 & 440 & 251 & 285 & 0.88 & -0.107 & 0.013 \\
\hline & & 5 & -0.104 & 0.047 & 384 & 218 & 285 & 0.77 & & \\
\hline & & 5 & -0.107 & 0.035 & 384 & 218 & 285 & 0.77 & & \\
\hline & & 5 & -0.079 & 0.035 & 384 & 218 & 285 & 0.77 & & \\
\hline & & 5 & -0.111 & 0.036 & 384 & 218 & 285 & 0.77 & -0.100 & 0.019 \\
\hline & & 6 & -0.137 & 0.026 & 420 & 239 & 285 & 0.84 & & \\
\hline & & 6 & -0.157 & 0.063 & 420 & 239 & 285 & 0.84 & & \\
\hline & & 6 & -0.124 & 0.029 & 420 & 239 & 285 & 0.84 & & \\
\hline & & 6 & -0.119 & 0.032 & 420 & 239 & 285 & 0.84 & -0.130 & 0.016 \\
\hline & & 7 & -0.074 & 0.031 & 432 & 246 & 285 & 0.86 & & \\
\hline & & 7 & -0.124 & 0.061 & 432 & 246 & 285 & 0.86 & & \\
\hline & & 7 & -0.087 & 0.035 & 432 & 246 & 285 & 0.86 & & \\
\hline & & 7 & -0.093 & 0.045 & 432 & 246 & 285 & 0.86 & -0.087 & 0.020 \\
\hline & \multirow[t]{23}{*}{ WPR-1, DS before chemistry } & 1 & -0.033 & 0.053 & 462 & 264 & 285 & 0.93 & & \\
\hline & & 1 & -0.059 & 0.049 & 462 & 265 & 285 & 0.93 & & \\
\hline & & 1 & -0.089 & 0.049 & 462 & 264 & 285 & 0.93 & & \\
\hline & & 1 & -0.117 & 0.051 & 462 & 264 & 285 & 0.93 & -0.075 & 0.025 \\
\hline & & 2 & -0.090 & 0.100 & 488 & 278 & 285 & 0.98 & & \\
\hline & & 2 & -0.046 & 0.050 & 488 & 278 & 285 & 0.98 & & \\
\hline & & 2 & -0.098 & 0.054 & 488 & 278 & 285 & 0.98 & & \\
\hline & & 2 & -0.094 & 0.038 & 488 & 278 & 285 & 0.98 & -0.082 & 0.026 \\
\hline & & 3 & -0.106 & 0.062 & 448 & 255 & 285 & 0.89 & & \\
\hline & & 3 & -0.096 & 0.043 & 448 & 255 & 285 & 0.89 & & \\
\hline & & 3 & -0.106 & 0.052 & 448 & 255 & 285 & 0.89 & & \\
\hline & & 3 & -0.141 & 0.038 & 448 & 255 & 285 & 0.89 & -0.116 & 0.023 \\
\hline & & 4 & -0.022 & 0.049 & 422 & 242 & 285 & 0.85 & & \\
\hline & & 4 & -0.039 & 0.054 & 422 & 242 & 285 & 0.85 & & \\
\hline & & 4 & -0.016 & 0.053 & 422 & 242 & 285 & 0.85 & -0.025 & 0.030 \\
\hline & & 5 & -0.034 & 0.050 & 403 & 230 & 285 & 0.81 & & \\
\hline & & 5 & -0.022 & 0.050 & 403 & 230 & 285 & 0.81 & -0.028 & 0.035 \\
\hline & & 6 & -0.046 & 0.053 & 458 & 262 & 285 & 0.92 & & \\
\hline & & 6 & -0.050 & 0.047 & 458 & 262 & 285 & 0.92 & & \\
\hline & & 6 & -0.012 & 0.048 & 458 & 262 & 285 & 0.92 & -0.036 & 0.028 \\
\hline & & 7 & -0.094 & 0.048 & 483 & 276 & 285 & 0.97 & & \\
\hline & & 7 & -0.048 & 0.048 & 483 & 276 & 285 & 0.97 & & \\
\hline & & 7 & -0.050 & 0.055 & 483 & 276 & 285 & 0.97 & -0.065 & 0.029 \\
\hline
\end{tabular}


Table 3.4: Pt stable isotope results for individual analyses, with weighted means for each replicate as given in the main text (continued).

\begin{tabular}{|c|c|c|c|c|c|c|c|c|c|c|}
\hline Group & Sample & Replicate \# & $\delta^{198} \mathrm{Pt}$ & $\begin{array}{c}\text { internal } \\
\text { error } \\
(2 \mathrm{se})\end{array}$ & $\begin{array}{c}\mathrm{ng} \mathrm{Pt} \\
\text { (natural) }\end{array}$ & $\begin{array}{l}\text { apparent Pt } \\
\text { conc. (ng/g) }\end{array}$ & $\begin{array}{l}\text { ref. conc } \\
\text { (ng/g) }\end{array}$ & $\begin{array}{c}\text { meas. / } \\
\text { ref. }\end{array}$ & $\begin{array}{c}\text { weighted } \\
\text { mean }\end{array}$ & \pm \\
\hline \multicolumn{11}{|c|}{ Ores (continued) } \\
\hline & SARM-76 & 1 & -0.018 & 0.036 & 3955 & 3867 & 3590 & 1.08 & & \\
\hline & & 1 & -0.080 & 0.039 & 3954 & 3867 & 3590 & 1.08 & & \\
\hline & & 1 & -0.052 & 0.046 & 3954 & 3867 & 3590 & 1.08 & & \\
\hline & & 1 & -0.050 & 0.033 & 3955 & 3868 & 3590 & 1.08 & & \\
\hline & & 1 & -0.073 & 0.044 & 3955 & 3868 & 3590 & 1.08 & & \\
\hline & & 1 & -0.077 & 0.044 & 3955 & 3868 & 3590 & 1.08 & & \\
\hline & & 1 & -0.042 & 0.041 & 3955 & 3868 & 3590 & 1.08 & & \\
\hline & & 1 & -0.053 & 0.039 & 3955 & 3868 & 3590 & 1.08 & & \\
\hline & & 1 & -0.034 & 0.046 & 3955 & 3868 & 3590 & 1.08 & -0.052 & 0.013 \\
\hline \multirow[t]{11}{*}{ Mantle } & OKUM & 1 & -0.133 & 0.052 & 157 & 10.4 & 11.44 & 0.91 & & \\
\hline & & 1 & -0.153 & 0.041 & 157 & 10.4 & 11.44 & 0.91 & & \\
\hline & & 1 & -0.148 & 0.079 & 157 & 10.4 & 11.44 & 0.91 & -0.146 & 0.030 \\
\hline & & 2 & -0.240 & 0.039 & 149 & 9.8 & 11.44 & 0.86 & & \\
\hline & & 2 & -0.185 & 0.073 & 149 & 9.8 & 11.44 & 0.86 & -0.228 & 0.034 \\
\hline & & 3 & -0.130 & 0.140 & 154 & 10.2 & 11.44 & 0.89 & & \\
\hline & & 3 & -0.164 & 0.059 & 154 & 10.2 & 11.44 & 0.89 & -0.159 & 0.054 \\
\hline & JP-1 & 1 & -0.328 & 0.040 & 77 & 5.1 & 5 & 1.02 & & \\
\hline & & 1 & -0.380 & 0.100 & 77 & 5.1 & 5 & 1.02 & -0.335 & 0.037 \\
\hline & PCC-1 & 1 & -0.309 & 0.057 & 96 & 7.9 & 8 & 0.98 & & \\
\hline & & 1 & -0.430 & 0.150 & 96 & 7.9 & 8 & 0.98 & -0.324 & 0.053 \\
\hline \multirow[t]{21}{*}{ Igneous } & SARM-6 & 1 & -0.151 & 0.035 & 587 & 39.1 & 39 & 1.00 & & \\
\hline & & 1 & -0.158 & 0.032 & 587 & 39.1 & 39 & 1.00 & & \\
\hline & & 1 & -0.143 & 0.043 & 587 & 39.1 & 39 & 1.00 & & \\
\hline & & 1 & -0.100 & 0.045 & 587 & 39.1 & 39 & 1.00 & & \\
\hline & & 1 & -0.099 & 0.052 & 587 & 39.1 & 39 & 1.00 & & \\
\hline & & 1 & -0.208 & 0.055 & 594 & 39.5 & 39 & 1.01 & -0.144 & 0.017 \\
\hline & & 2 & -0.137 & 0.050 & 551 & 36.2 & 39 & 0.93 & & \\
\hline & & 2 & -0.101 & 0.045 & 551 & 36.2 & 39 & 0.93 & & \\
\hline & & 2 & -0.113 & 0.040 & 551 & 36.2 & 39 & 0.93 & & \\
\hline & & 2 & -0.143 & 0.041 & 551 & 36.2 & 39 & 0.93 & & \\
\hline & & 2 & -0.142 & 0.038 & 551 & 36.2 & 39 & 0.93 & & \\
\hline & & 2 & -0.154 & 0.038 & 551 & 36.2 & 39 & 0.93 & -0.133 & 0.017 \\
\hline & BIR-1 & 1 & -0.407 & 0.058 & 64 & 4.2 & 4.3 & 0.98 & & \\
\hline & & 1 & -0.270 & 0.110 & 64 & 4.2 & 4.3 & 0.98 & -0.377 & 0.051 \\
\hline & DNC-1 & 1 & -0.056 & 0.041 & 534 & 35.6 & 36.8 & 0.97 & & \\
\hline & & 1 & -0.070 & 0.040 & 534 & 35.6 & 36.8 & 0.97 & & \\
\hline & & 1 & -0.047 & 0.029 & 534 & 35.6 & 36.8 & 0.97 & & \\
\hline & & 1 & -0.043 & 0.047 & 534 & 35.6 & 36.8 & 0.97 & & \\
\hline & & 1 & -0.024 & 0.052 & 534 & 35.6 & 36.8 & 0.97 & -0.050 & 0.018 \\
\hline & LK-NIP & 1 & -0.213 & 0.036 & 186 & 12.3 & 13.43 & 0.91 & & \\
\hline & & 1 & -0.219 & 0.044 & 186 & 12.3 & 13.43 & 0.91 & -0.215 & 0.028 \\
\hline \multirow[t]{2}{*}{ Other } & FC-1 & 1 & -0.011 & 0.053 & 120 & 30.4 & 38 & 0.80 & & \\
\hline & & 1 & -0.037 & 0.074 & 120 & 30.4 & 38 & 0.80 & -0.020 & 0.043 \\
\hline
\end{tabular}




\section{Chapter 4}

\section{Platinum stable isotope tracing of Earth's accretion and differentiation}

This chapter has been prepared as a draft manuscript for submission to Earth and Planetary Science Letters*, and has been formatted according to the requirements of that journal. The article is entitled Platinum stable isotope tracing of Earth's accretion and differentiation. References have been gathered to the combined references section at the end of this thesis.

(

\section{Abstract}

Highly siderophile elements (HSE) are strongly partitioned into the cores of terrestrial planets during core formation, and the abundances of HSE in Earth's mantle compared with primitive meteorites have provided key constraints on models of Earth's early evolution. Two leading models to explain the HSE abundances in the silicate Earth involve a late-veneer of

* Author list: John Creech, Joel Baker, Monica Handler and Martin Bizzarro 
chondritic material that was added after core formation, or core formation in a deep magma ocean. Platinum (Pt) is a HSE, and the newly developed platinum $(\mathrm{Pt})$ stable isotope system represents a novel tool for investigating these processes.

We report $\mathrm{Pt}$ stable isotope results for enstatite, ordinary and carbonaceous chondrites, primitive achondrites, iron meteorites and terrestrial mantle samples measured by double-spike MC-ICPMS. Results are expressed relative to the IRMM-010 Pt isotope standard as the per mill difference in ${ }^{198} \mathrm{Pt} /{ }^{194} \mathrm{Pt}$ ratios $\left(\delta^{198} \mathrm{Pt}\right)$. Our data set reveals that the $\mathrm{Pt}$ stable isotopic composition of Earth's mantle $\left(\delta^{198} \mathrm{Pt}_{\text {mantle }}=-0.27 \pm 0.15 \%\right.$ overlaps with chondrites from all groups $\left(\delta^{198} \mathrm{Pt}_{\text {chondrites }}=-0.14 \pm 0.10 \%\right.$ o $)$. Ordinary and enstatite chondrite samples show little variation. Carbonaceous chondrites have an average composition similar to the other groups of chondrites but show greater variability, potentially relating to isotopic differences among the components of these meteorites. Primitive achondrite and ureilite samples revealed the heaviest compositions of all meteorite groups $\left(\delta^{198} \mathrm{Pt}=-0.08 \%\right.$ o to $0.00 \% 0$ ). These data suggest that metal-silicate differentiation produces an isotopic fractionation for $\mathrm{Pt}$, with heavy isotopes being preferentially retained in the silicate phase. Thus, Earth's mantle would be expected to have been significantly enriched in the heavy isotopes of Pt during core formation. The absence of a large fractionation between chondrites, representing the composition of the undifferentiated Earth, and the mantle suggests that the fingerprint of core formation has been overprinted in Earth's mantle. Therefore, Pt isotope data is consistent with a late-veneer of chondritic material added to Earth after the core had formed. A small but statistically significant difference between the composition of the mantle and chondrites may reflect isotopic fractionation during within-mantle processes. 


\subsection{Introduction}

Platinum (Pt) is a highly siderophile transition metal that has five stable isotopes - ${ }^{192} \mathrm{Pt},{ }^{194} \mathrm{Pt},{ }^{195} \mathrm{Pt},{ }^{196} \mathrm{Pt}$ and ${ }^{198} \mathrm{Pt}$ - with relative abundances of $\sim 0.79 \%, 32.81 \%, 33.79 \%, 25.29 \%$ and $7.31 \%$, respectively (Creech et al. 2013 . It can exist in a range of oxidation states, but principally occurs as $\mathrm{Pt}^{0}, \mathrm{Pt}^{2+}$ and $\mathrm{Pt}^{4+}$ (Cotton and Wilkinson, 1966). The relatively large mass difference (ca. $2 \%$ ) between the abundant heavy and light isotopes of Pt, coupled with its variable oxidation states and strong elemental partitioning between metals and silicates makes the Pt stable isotope system uniquely suited to tracing processes of Earth's accretion and differentiation. It has recently been shown that $\mathrm{Pt}$ stable isotope compositions can be measured in natural samples to a precision on $\delta^{198} \mathrm{Pt}$ of $\leq 0.02 \%$ omu $\mathrm{amd}^{-1}$ and that is sufficient to resolve natural isotopic variations in terrestrial materials (Creech et al. 2014$)^{2}$. This study measures Pt stable isotopic variations in terrestrial and meteoritic samples in order to investigate processes during Earth's early accretion and differentiation.

Highly siderophile element (HSE; Rh, Ru, Pd, Os, Ir, Pt, $\mathrm{Au}$ and $\mathrm{Re}$ ) abundances in Earth's mantle have played a key role in constraining models of Earth's accretion and differentiation. HSE concentrations in the mantle are significantly depleted relative to chondrites, reflecting their extraction into the core during Earth's differentiation. However, mantle abundances of HSE are orders of magnitude too high to be consistent with equilibrium core formation at low pressures and temperatures (e.g., Ringwood, 1966, Chou, 1978; Jagoutz et al., 1979, Righter, 2003). The two prevailing models that may explain the apparent excess of HSE remaining in the mantle consider either late addition of meteoritic material or equilibrium core formation in a magma ocean. The 'late-veneer' hypothesis proposes that ca. $0.1 \%-1.0 \%$ of

\footnotetext{
${ }^{1}$ Citations to Creech et al. (2013) in this chapter refer to the material presented in Chapter 2 of this thesis.

${ }^{2}$ Citations to Creech et al. (2014) in this chapter refer to the material presented in Chapter 3 of this thesis.
} 
Earth's mass of chondritic material was accreted to Earth after core formation was complete (Kimura et al., 1974, Chou, 1978; Holzheid et al., 2000. Dauphas and Marty, 2002). This late-veneer of relatively HSE-rich material was subsequently mixed into the mantle, and in addition to explaining the observed absolute HSE abundances in the mantle, this model also explains the relative abundances of HSE in the mantle, which are broadly chondritic (Mitchell and Keays, 1981; Rehkämper et al., 1997; Handler and Bennett 1999), and potentially provides a source for Earth's volatile element budget (Drake and Righter, 2002; Albarède, 2009, Schönbächler et al., 2010; Wang and Becker, 2013). However, recent HSE metal-silicate partitioning data suggest that under different physical conditions (temperature, pressure and oxygen fugacity) some elements become significantly less siderophile. These models suggest that core formation took place in a deep magma ocean and, thus, more of the HSE were retained in the mantle after core formation, potentially obviating the need for a chondritic late-veneer.

These processes are likely to have imparted different Pt stable isotope signatures onto Earth's mantle. The late-veneer has been argued to comprise carbonaceous (Chou, 1978; Morgan, 1986, Morbidelli et al., 2000, Dauphas et al., 2004a), enstatite (Shirey and Walker, 1998; Walker et al., 2001; Meisel et al. 1996; Righter, 2003 Fischer-Gödde et al., 2011) or ordinary (Meisel et al., 1996; Shirey and Walker, 1998; Walker et al., 2001; Drake and Righter, 2002 Fischer-Gödde et al., 2011) chondrite material, or combinations of these. If the HSE in Earth's mantle were delivered by a late-veneer, the Pt stable isotopic composition of the mantle should therefore be similar to that of chondrites, and possibly of a particular group.

Alternatively, if the HSE abundances in the mantle are a product of metal-silicate differentiation in a magma ocean, this may lead to a distinct isotopic composition of the mantle. The Earth is considered to have a bulk composition similar to chondrites, thus chondritic composition should also establish the composition of the undifferentiated Earth (i.e., prior to core formation), providing the starting composition against which equilib- 
rium isotopic fractionations in a magma ocean should be referenced. Published metal-silicate partition coefficients for Pt vary from $>10^{9}$ at low pressures and moderate temperatures (e.g., $1300^{\circ} \mathrm{C}, 1$ bar; Ertel et al., 1999) to ca. $10^{3}-10^{4}$ at high pressures and temperatures (e.g., $1400{ }^{\circ} \mathrm{C}-1900{ }^{\circ} \mathrm{C}$, 0.5-14 GPa; Ertel et al. 2006). Thus, even under high pressure and temperature (magma ocean) conditions, ca. 99.99\% of Earth's Pt budget should have been partitioned into the core. Given this extreme Pt partitioning and the presumed change of oxidation state between the silicate mantle $\left(\mathrm{Pt}^{2+}\right)$ and $\mathrm{Fe}-\mathrm{Ni}$ metallic core $\left(\mathrm{Pt}^{0}\right)$, a fractionation of $\mathrm{Pt}$ isotopes may be expected. Such a fractionation should be apparent as an offset in isotopic compositions between the present day mantle and the undifferentiated Earth (i.e., chondrite) composition. While stable isotopic fractionations at such high temperatures and pressures are expected to be small, measurable fractionations inferred to result from similar processes have been reported in other non-traditional stable isotope systems (e.g., Si, Cr, S; Georg et al., 2007; Fitoussi et al., 2009; Shahar et al., 2009, 2011; Armytage et al., 2011; Moynier et al., 2011; Labidi et al., 2013).

In addition to data from chondritic meteorites, further constraints on the Pt stable isotopic fractionations during planetary differentiation may come from primitive achondrites, ureilites and iron meteorites. Primitive achondrites and ureilites sample planetary bodies that have experienced different degrees of melting and possibly metal-silicate differentiation. These types of meteorites should thus provide constraints on the magnitude as well as the direction of isotopic fractionations resulting from these processes. Iron meteorites sample the cores of differentiated planetesimals, and although these bodies undoubtedly formed under very different physical conditions than Earth's core, iron meteorite data will yield information about the extent of $\mathrm{Pt}$ stable isotope variation between the cores of their respective parent bodies and the material from which they accreted.

This study reports new Pt stable isotope data for Earth's mantle and chondrites, primitive achondrites, ureilites and iron meteorites. The implica- 
tions of these data in the context of Earth's early history are then discussed.

\subsection{Samples and methods}

\subsubsection{Terrestrial and meteorite samples}

Two peridotite mantle xenolith samples from northern Queensland, Australia (BC-1 and MQ-14) were measured in this study. BC-1 is a peridotite from Batchelor's Crater, which is a Plio-Pleistocene alkali basalt vent in the Chudleigh volcanic province, north Queensland, Australia. MQ-14 is a peridotite xenolith from Mount Quincan in the $<3$ Ma Atherton Tablelands Volcanic Province. Major and trace element and Sm-Nd isotopic data for a suite of peridotite xenoliths from this locality, including MQ-14, were presented by (Handler et al. 2005). The Mount Quincan xenolith suite represent a fertile mantle source with a ${ }^{147} \mathrm{Sm}-{ }^{143} \mathrm{Nd}$ age of ca. $275 \mathrm{Ma}$ (Handler et al. 2005). These two mantle samples add to the terrestrial database of Pt stable isotope data published by Creech et al. (2014), which are also used in the discussion later in the paper.

Eleven chondrite meteorites were analysed in this study, comprising three ordinary chondrites, one enstatite chondrite and seven carbonaceous chondrites (Table 4.1). Five iron meteorites, comprising two IAB, one IIIAB, one IVA and one IVB, were also measured in this study (Table 4.1). Previously published cosmic ray exposure (CRE) ages for these meteorites also are given in Table 4.1. Two acapulcoite primitive achondrites and one ureilite specimen were also analysed (Table 4.1). Further details about these meteorites can be obtained from the Meteoritical Bulletin Database (http://www.lpi.usra.edu/meteor $/)^{3}$

\footnotetext{
${ }^{3}$ Additional sample information and specimen photographs are also presented in Appendix $\mathrm{A}$ of this thesis.
} 


\subsubsection{Analytical methods}

The techniques used in this study are described in detail by Creech et al. (2013, 2014).

All sample preparation and digestions were carried out in an ultra-clean laboratory at Victoria University of Wellington, New Zealand. Digestions of stony (chondrite, primitive achondrite and ureilite) meteorites and terrestrial samples were carried out using a nickel sulphide fire assay method as described by Creech et al. (2014). Previously powdered material was available for the terrestrial peridotites samples and these were weighed directly into porcelain crucibles for NiS digestion. Fragments of stony meteorites were taken from sliced specimens, weighed and then cleaned by rinsing in ultrapure water, methanol and again in ultra-pure water. The meteorite fragments were then placed in a Savillex teflon beaker in ca. $4 \mathrm{~mL}$ of $2 \mathrm{M} \mathrm{HCl}$. The beakers were heated to $120^{\circ} \mathrm{C}$ for ca. $10 \mathrm{~min}$ and then ultrasonicated for ca. 3 min. After this gentle acid washing, the chondrites were rinsed twice with water and then dried in the beaker on a hotplate. The chondrites were then crushed into a fine powder in an agate mortar and weighed before being transferred to porcelain crucibles for NiS fire assay digestion. A ${ }^{196} \mathrm{Pt}-{ }^{198} \mathrm{Pt}$ double-spike (DS) was added to samples prior to NiS digestion by adding the Pt DS to the mixture in the crucible for approximate proportions of $55 \% \mathrm{Pt}$ from DS and $45 \%$ natural Pt, and allowing to dry before being fused in a furnace. The preparation and fusion of NiS charges and dissolution of NiS beads were performed as described by Creech et al. (2014).

Digestion of iron meteorite samples was carried out by acid digestion. Small fragments of $\mathrm{Fe}-\mathrm{Ni}$ metal weighing ca. $0.5 \mathrm{~g}$ were cut from larger iron meteorite specimens using a diamond cutting disc attached to a small handheld drill. Surface contamination and oxidation were removed by abrasion with fine-grained $\mathrm{SiC}$ sandpaper, and fragments were washed in ultra-pure water and cleaned with methanol to remove any material acquired during handling. The metal was then acid washed by adding ca. $4 \mathrm{~mL}$ of $1.5 \mathrm{M}$ $\mathrm{HCl}$ at room temperature and placing it in an ultrasonic bath for a few 
minutes, and then by placing it in ca. $4 \mathrm{~mL}$ of $0.8 \mathrm{M} \mathrm{HNO}_{3}$ on a hotplate at $120^{\circ} \mathrm{C}$ for up to $30 \mathrm{~min}$ before rinsing with ultra-pure water and drying. Iron meteorite samples were double-spiked prior to dissolution of the iron meteorite fragments to have proportions of approximately $55 \%$ of $\mathrm{Pt}$ from DS and $45 \%$ natural Pt. The Fe-Ni metal (with the added DS) was then digested in aqua regia on a hotplate at $120^{\circ} \mathrm{C}$. Once fully digested, the samples were evaporated to dryness, converted to chloride form by evaporating with concentrated $\mathrm{HCl}$, and finally brought into solution in $10 \mathrm{~mL}$ of $0.5 \mathrm{M} \mathrm{HCl}$ for anion-exchange chemistry. The sample and DS were taken to be fully equilibrated after this procedure.

Purification of $\mathrm{Pt}$ from samples was performed using the anion-exchange chemical methods described by Creech et al. (2014), using AG1-X8 (100-200 mesh) anion exchange resin. The major cationic species in samples are not retained on this resin, and the platinum group elements were eluted using sequentially stronger $\mathrm{HCl}$ and $\mathrm{HNO}_{3}$ acids. Pt is collected in a final step using concentrated $\mathrm{HNO}_{3}$, with a Pt yield of $\geq 90 \%$ and purity of $\geq 95 \%$ (Creech et al. 2014). This level of Pt separation has been shown to be adequate for accurate determination of $\mathrm{Pt}$ isotope compositions by double-spike MCICPMS (Creech et al. 2013). All Pt separation work was conducted in class 10 laminar flow hoods situated in a class 100 clean laboratory. In order to destroy any potential organic interferences, Pt cuts were treated by refluxing in aqua regia overnight on a hotplate, evaporating to dryness, refluxing in $\mathrm{HNO}_{3}-\mathrm{H}_{2} \mathrm{O}_{2}$ overnight on a hotplate, and then evaporating to dryness again. Samples were then reconverted to chloride form by evaporating concentrated $\mathrm{HCl}$ before being taken up in $0.5 \mathrm{M} \mathrm{HCl}$ for measurement by MC-ICPMS.

Pt stable isotope measurements were carried out using the techniques described by Creech et al. (2013), which utilises a ${ }^{196} \mathrm{Pt}-{ }^{198} \mathrm{Pt}$ DS to correct for instrumental mass fractionation. Isotopic measurements were made using a Nu Plasma MC-ICPMS at Victoria University of Wellington, New Zealand, under identical conditions to those described by Creech et al. (2013). All data reduction was conducted offline using the double-spike data reduction 
tool IsoSpike (Creech and Paul, 2014), which is an add-on for the Iolite data reduction package (www.iolite.org.au). IsoSpike is open-source and freely available from www.isospike.org. Pt concentrations of samples were also calculated offline using isotope dilution calculations as described by Creech et al. (2014).

\subsection{Results}

Pt stable isotope results are summarised in Table 4.1 and Figure 4.1. Table 4.1 also shows measured Pt concentrations for each sample determined from isotope dilution calculations. Although the reproducibility of $\delta^{198} \mathrm{Pt}$ measurements of meteorite samples was generally ca. $\leq \pm 0.04-0.05 \%$ o ( 2 sd; Table 4.1), which is similar to the reproducibility of the technique as demonstrated by (Creech et al., 2013), measurements of repeat digestions of a terrestrial standard using the NiS fire assay were shown by Creech et al. 2014 to have a reproducibility on $\delta^{198} \mathrm{Pt}$ of $\pm 0.08 \%$. We have therefore conservatively assumed an uncertainty of $\pm 0.08 \%$ o for all NiS digested samples (Figure 4.1).

The two mantle xenolith samples, BC-1 and MQ-14, yield $\delta^{198} \mathrm{Pt}$ values of $-0.25 \%$ and $-0.17 \%$ o, respectively, which are both within the compositional range for mantle derived samples of ca. $-0.34 \%$ o to $-0.18 \%$ o (which includes the komatiite standard OKUM) presented by Creech et al. (2014).

Pt stable isotope compositions from enstatite and ordinary chondrite samples are identical within estimated uncertainties $\left(\delta^{198} \mathrm{Pt} \approx-0.15 \%\right.$ o $)$. Based on the three ordinary chondrite samples analysed in this study, this group of chondrites may be isotopically homogeneous for Pt. Greater variability was observed amongst the seven carbonaceous chondrite samples, with $\delta^{198} \mathrm{Pt}$ values ranging from ca. $-0.24 \%$ o to $-0.08 \%$, although the mean value for the carbonaceous chondrites $(-0.14 \% 0)$ is essentially identical to that of the ordinary and enstatite chondrites $(-0.15 \%$ ). The mean of all of the chondrite samples gives a chondritic composition of $\delta^{198} \mathrm{Pt}_{\text {chondrite }}=-0.14 \pm$ $0.10 \%$ o $(\mathrm{n}=11 ; 2 \mathrm{sd})$. 
Table 4.1: Pt stable isotope and isotope dilution results for terrestrial and extraterrestrial samples measured by double-spike MC-ICPMS relative to the IRMM-010 Pt isotope standard. ${ }^{*}$ Pt stable isotope compositions given are weighted means of the number of analyses $(n)$ of each digestion, with the uncertainties reflecting the errors on the weighted means.

\begin{tabular}{lcccc}
\hline Sample & $\begin{array}{c}\delta^{198} \mathrm{Pt} \\
(\%)\end{array}$ & $(\mathrm{n})$ & $\begin{array}{c}\text { Pt conc. } \\
\left(\mu \mathrm{g} \mathrm{g}^{-1}\right)\end{array}$ & $\begin{array}{c}\text { Additional } \\
\text { information }^{\ddagger}\end{array}$ \\
\hline Terrestrial peridotites & & & & \\
BC-1 & $-0.25 \pm 0.04$ & 2 & 0.0068 & \\
MQ-14 & $-0.17 \pm 0.03$ & 2 & 0.0068 & $\sim 3.0^{b}$ \\
JP-1 & $-0.34 \pm 0.04$ & 2 & 0.0051 & $0.64^{c}$ \\
PCC-1 $^{a}$ & $-0.32 \pm 0.05$ & 2 & 0.0079 & $0.68^{c}$ \\
Ordinary chondrites & & & & \\
Begaa (LL3) & $-0.15 \pm 0.03$ & 3 & 0.52 & \\
Bovedy (L3) & $-0.15 \pm 0.03$ & 3 & 1.00 & \\
Talbachat n'aït Isfoul (LL3) & $-0.15 \pm 0.02$ & 7 & 0.30 & \\
Enstatite chondrites & & & & \\
SAH 97096 (EH3) & $-0.15 \pm 0.03$ & 4 & 1.33 & \\
Carbonaceous chondrites & & & & \\
Gujba (CB3) & $-0.08 \pm 0.04$ & 3 & 4.42 & \\
NWA 1232 (CO3) & $-0.08 \pm 0.03$ & 3 & 0.98 & \\
NWA 763 (CO3) & $-0.17 \pm 0.04$ & 3 & 1.02 & \\
NWA 1559 (CK3) & $-0.17 \pm 0.04$ & 4 & 0.49 & \\
NWA 1563 (CK5) & $-0.08 \pm 0.03$ & 4 & 0.68 & \\
NWA 723 (CV3) & $-0.24 \pm 0.03$ & 3 & 1.16 & \\
SaU 290 (CH3) & $-0.16 \pm 0.03$ & 3 & 0.78 & \\
Achondrites & & & & \\
Dho 125 (acapulcoite) & $-0.09 \pm 0.02$ & 4 & 1.45 & \\
NWA 2871 (acapulcoite) & $-0.04 \pm 0.03$ & 3 & 2.27 & \\
NWA 2234 (ureilite) & $0.00 \pm 0.04$ & 4 & 0.26 & \\
Iron meteorites & & & & \\
Chinga (IVB) & $-0.22 \pm 0.02$ & 7 & 7.61 & $845^{c}$ \\
Gibeon (IVA) & $-0.19 \pm 0.01$ & 7 & 3.90 & $400^{d}$ \\
Henbury (IIIAB) & $-0.31 \pm 0.03$ & 2 & 15.24 & $700^{e}$ \\
Toluca (IAB) & $-0.18 \pm 0.03$ & 2 & 7.30 & $600^{f}$ \\
Canyon Diablo (IAB) & $-0.24 \pm 0.05$ & 1 & 6.09 & $540^{g}$ \\
\hline
\end{tabular}

${ }^{*}$ Dho, NWA, SAH and SaU are abbreviations for the regions Dhofar, Northwest Africa, Sahara, and Sayh al Uhaymir, respectively. ${ }^{\ddagger}$ Additional information for sample groups: peridotites, $\mathrm{Al}_{2} \mathrm{O}_{3}$ content in wt.\%, as an index of the degree of melt extraction; iron meteorites, CRE ages in Myr. ${ }^{a}$ data from Creech et al. (2014). ${ }^{b}$ based on typical values for Mount Quincan samples from Handler et al. (2005). ${ }^{c}$ calculated based on values from GeoRem database. ${ }^{d}$ weighted average for group from Scherstén et al. (2006). SScherstén et al. (2006). fMasarik (1997). s Michlovich et al. (1994).

Iron meteorites yield a mean composition of $\delta^{198} \mathrm{Pt}=-0.23 \pm 0.11 \%$ o $(\mathrm{n}=$ $5 ; 2 \mathrm{sd})$. With the exception of Henbury, the iron meteorite samples all have almost identical $\mathrm{Pt}$ isotopic compositions. Henbury has the lowest $\delta^{198} \mathrm{Pt}$ value amongst the iron meteorites of $-0.31 \%$. If the analysis of Henbury is 


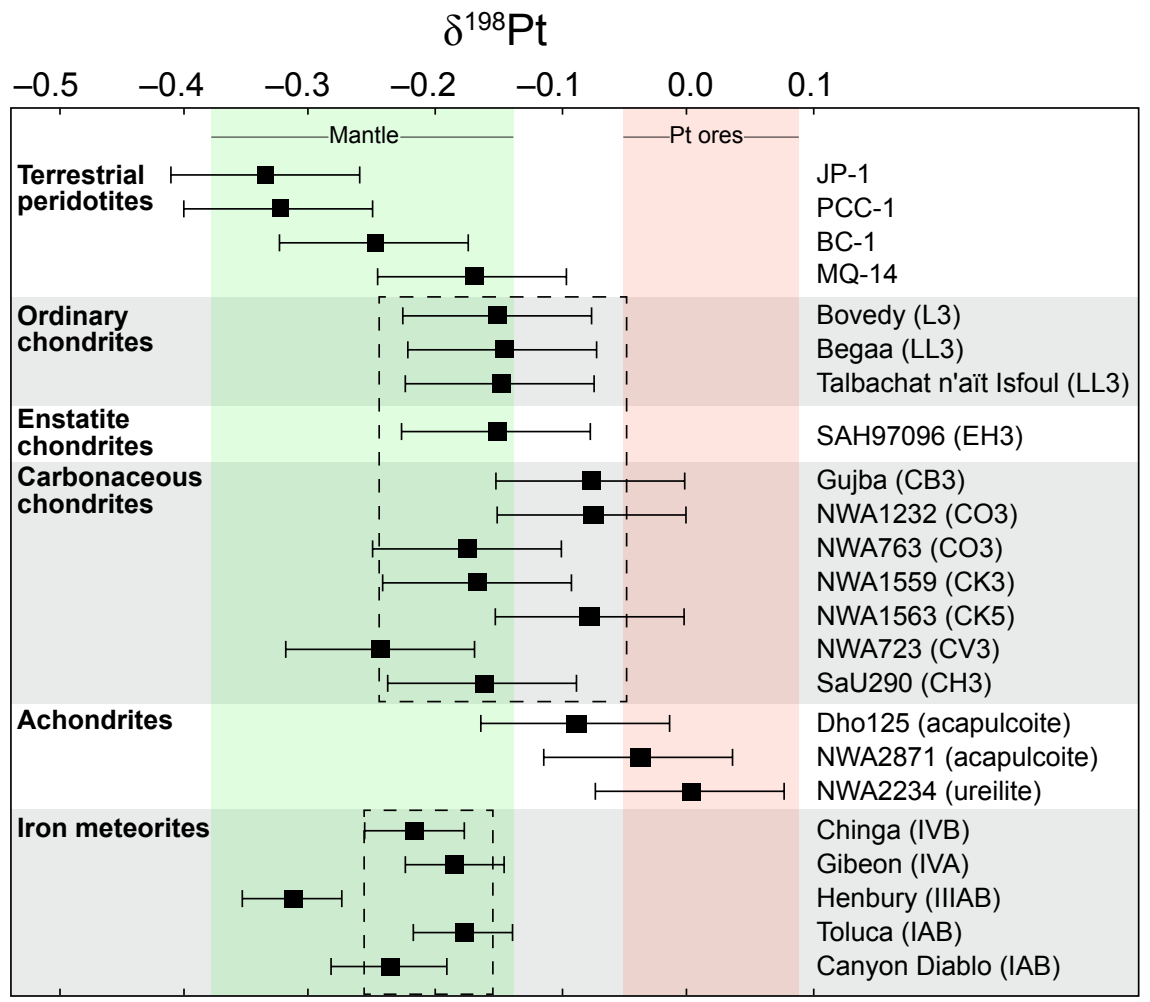

Figure 4.1: $\mathrm{Pt}$ stable isotope results for terrestrial and meteorite samples. Error bars for $\mathrm{NiS}$ digested samples are $\pm 0.08 \%$, which was shown to be the reproducibility of the current technique by Creech et al. (2014). Error bars on iron meteorite samples are $\pm 0.04 \%$, which is based on the reproducibility in solution standards as shown by Creech et al. (2013). The green and red shaded vertical boxes represent the compositional range of the mantle and $\mathrm{Pt}$ ores, respectively, based on data presented here and by Creech et al. (2014). Dashed boxes represent the $95 \%$ confidence interval for the respective meteorite groups they enclose. The 95\% confidence interval for the iron meteorites excludes Henbury, which, based on the present data set, appears to be an outlier.

excluded, the composition of the iron meteorites as a group is $\delta^{198} \mathrm{Pt}_{\text {irons }}=$ $-0.20 \pm 0.06 \%$ o (2 sd).

Primitive achondrites are isotopically heavier than all other groups of meteorites, with $\delta^{198} \mathrm{Pt}$ ranging from -0.09 to $0.00 \%$.

The stable $\mathrm{Pt}$ isotope compositional range exhibited by the meteorite samples is less than that of the terrestrial samples. 


\subsection{Discussion}

\subsubsection{The origin of $\mathrm{Pt}$ stable isotope variations within meteorite groups}

\section{Chondrites}

The carbonaceous chondrite samples span a wide range (ca. $0.17 \%$ ) in $\delta^{198} \mathrm{Pt}$, whereas the ordinary and enstatite chondrites occupy a narrow compositional range $(<0.01 \%$; Fig. 4.1). While further studies of ordinary and enstatite chondrites may show a wider range of compositions, the variations observed amongst carbonaceous chondrites may reflect real compositional differences that are more apparent in these samples due to the greater proportions of matrix and refractory inclusions (amoeboid olivine aggregates, Ca-Al-rich inclusions). Given a typical sample size of ca. $0.5 \mathrm{~g}$ meteorite fragments (i.e., a ca. 5-6 mm cube) it is not unlikely that individual samples taken from these specimens could unevenly sample the different components of carbonaceous chondrites, and larger samples may be required to accurately determine the bulk isotopic composition of carbonaceous chondrites. However, the mean $\delta^{198} \mathrm{Pt}$ value of carbonaceous chondrites is consistent with the other chondrite groups, suggesting that the bulk composition of carbonaceous chondrites is similar to the other chondrite groups. If the variability in the present data set does in fact reflect compositional variations amongst the different components within carbonaceous chondrites then sampling different phases in carbonaceous chondrites may reveal pre-solar Pt stable isotope variations or different nucleosynthetic inputs. Given the relatively short CRE ages typical of chondrites (e.g., Herzog, 2007), cosmogenic effects are not considered to have had significant effects on Pt stable isotope ratios.

\section{Iron meteorites}

The iron meteorites analysed in this study have a range of CRE ages (Table 4.1. It is important to consider mass independent isotopic effects from 
thermal neutron capture, particularly given that the DS-corrected data in this study did not include unspiked measurements.

Two recent studies have suggested using Pt isotope ratios as a dosimeter for correcting cosmogenic effects in Hf-W isotopic data (Kruijer et al., 2013. Wittig et al., 2013). These studies show large anomalies from production of ${ }^{192} \mathrm{Pt}$ by neutron capture on ${ }^{191} \mathrm{Ir}$. This result partially motivated the choice of the ${ }^{196} \mathrm{Pt}-{ }^{198} \mathrm{Pt}$ DS used in this study, as described by Creech et al. (2013). Of the five stable Pt isotopes, ${ }^{195} \mathrm{Pt}$ has the largest thermal neutron capture cross section of ca. 28 barns (Mughabghab, 2003), and given the isotopes used in the ${ }^{196} \mathrm{Pt}-{ }^{198} \mathrm{Pt}$ DS inversion $\left({ }^{194} \mathrm{Pt},{ }^{195} \mathrm{Pt},{ }^{196} \mathrm{Pt}\right.$ and $\left.{ }^{198} \mathrm{Pt}\right)$, neutron capture on ${ }^{195} \mathrm{Pt}$ has the greatest potential to compromise our results. A sensitivity test for DS calculations using the ${ }^{196} \mathrm{Pt}-{ }^{198} \mathrm{Pt}$ DS was performed whereby measured isotope ratios from a standard were modified by the magnitude of the anomalies reported for iron meteorites by Wittig et al. (2013), which are based on measurements of IVB irons with a range of CRE ages. With the most extreme anomalies reported by Wittig et al. (2013) (i.e., for the meteorite Tlacotepec), the effect on the calculated $\delta^{198} \mathrm{Pt}$ was ca. $0.22 \%$. However, using typical anomalies from Wittig et al. (2013) for most iron meteorites (i.e., $\varepsilon^{194} \mathrm{Pt} \sim 1$ ) the effect on Pt stable isotopic measurements is considerably smaller at ca. $0.04 \%$. With the exception of Henbury, the iron meteorite samples analysed here have relatively consistent $\mathrm{Pt}$ isotope ratios. This suggests that, despite the range of CRE ages in these samples, the DS-corrected Pt stable isotope ratios have not been significantly disturbed by mass independent effects. Henbury is offset from the other iron meteorite samples by ca. $0.10 \%$, which might be accounted for by cosmogenic effects. Further studies of iron meteorite samples, particularly where DS sample measurements include an unspiked run, are required to further constrain the magnitude of these effects, although for most irons these appear to be less than the currently achievable analytical uncertainties. Thus, based on the available data, the Pt isotopic composition of iron meteorites is constrained to ${ }^{198} \mathrm{Pt}_{\text {irons }}=-0.20 \pm 0.06 \%$ o $(2 \mathrm{sd}, \mathrm{n}=4)$. 


\subsubsection{Pt stable isotope composition of Earth's mantle}

The Pt stable isotopic compositions of xenolith peridotites BC-1 and MQ14 are consistent with the compositional range of the mantle determined from mantle and mantle-derived standard reference materials by Creech et al. (2014) of -0.34 to $-0.18 \%$. Combined with the previously published data, these data define a relatively broad range in $\mathrm{Pt}$ isotopic composition for the mantle of $\delta^{198} \mathrm{Pt}_{\text {mantle }}=-0.27 \pm 0.15 \%$. These results suggest that the mantle is isotopically heterogeneous for Pt. It is not yet clear whether the heterogeneity in $\mathrm{Pt}$ stable isotopes is a primary feature of the mantle, or reflects secondary processes such as melt extraction or mantle metasomatism.

The elemental composition of the primitive upper mantle (PUM) is commonly determined by extrapolation from the most primitive peridotite samples, as defined by parameters such as $\mathrm{Al}_{2} \mathrm{O}_{3}$ content or $\mathrm{Mg} \#$, to the presumed composition of the mantle for those parameters. The peridotite samples MQ14, analysed in this study, and JP-1 and PCC-1 from Creech et al. (2014) show a correlation between $\mathrm{Al}_{2} \mathrm{O}_{3}$ content (i.e., the degree of melt extraction) and Pt stable isotope ratios (Table 4.1), with the most fertile mantle sample MQ14 having the heaviest (least negative) isotopic composition and the more depleted samples (JP-1 and PCC-1) having the lightest compositions. Given the small number of samples, little confidence can yet be placed in this correlation for the interpretations made below. However, if supported by the addition of future data, this observation could suggest (1) $\mathrm{Pt}$ stable isotope ratios in ultramafic and mafic rocks may be useful in investigating igneous processes such as melt extraction, magma differentiation and fractional crystallisation, and (2) the Pt stable isotopic composition of the primitive upper mantle is currently best constrained by the sample MQ14, which has an isotopic composition of $\delta^{198} \mathrm{Pt}=-0.17 \%$, which is virtually identical to the mean chondrite value. However, these interpretations are not yet rigorously supported by the available data, and thus the $\delta^{198} \mathrm{Pt}_{\text {mantle }}=$ $-0.27 \pm 0.15 \%$ as defined by the compositions of all of the mantle peridotite samples is taken to represent the composition of Earth's mantle for the dis- 
cussion below.

\subsubsection{Implications of $\mathrm{Pt}$ stable isotopes for accretion models}

\section{Pt isotope signature of metal-silicate differentiation}

The extreme partitioning and redox state change of $\mathrm{Pt}$ between silicate $\left(\mathrm{Pt}^{2+}\right)$ and metals $\left(\mathrm{Pt}^{0}\right)$ might be expected to produce an isotopic fractionation during core formation. Given that Pt is expected to have been quantitatively removed into the cores of differentiated asteroids, the Pt stable isotopic composition of their cores (i.e. iron meteorites) should reflect that of the precursor material from which the body formed, with no significant isotopic fractionation resulting from core formation. The overlap in $\mathrm{Pt}$ stable isotope compositions of iron meteorites and chondrites is consistent with these bodies being formed from chondrite-like material. Given the leverage of the core component of the $\mathrm{Pt}$ budget, a significantly larger fractionation might be expected in the residual $\mathrm{Pt}$ remaining in the silicate mantle after core formation, which should be apparent as an offset between the mantle composition and that of chondrites. This hypothesis is supported by data from the acapulcoite and ureilite samples. Acapulcoites are thought to represent residues after extraction of low degree partial melts from an essentially chondritic composition (e.g., McCoy et al., 1997b), and ureilites, although they have a more complicated history, are generally considered to represent ultramafic rocks from which have been partially melted and a basaltic component has been extracted (Goodrich et al., 2004, Mittlefehldt, 2007. Rankenburg et al. 2008). Thus, these samples represent planetary bodies that only underwent the earliest stages of melting and metal-silicate differentiation from chondrite-like material. Acapulcoites yield Pt isotopic compositions that are heavier than chondrites by ca. $0.06-0.10 \%$, and the ureilite sample yielded the heaviest Pt isotopic composition of any meteorite sample in this study, ca. $0.15 \%$ o heavier than the mean chondrite value. Based on 
this limited number of samples, this trend suggests that metal-silicate differentiation did produce an isotopic fractionation, where the lighter isotopes of $\mathrm{Pt}$ were preferentially incorporated in the metal phase and the heavier isotopes were concentrated in the residual $\mathrm{Pt}$ in the mantle. This contrasts with the direction of fractionation observed between the mantle and $\mathrm{Pt}$ ores, where the mantle is isotopically light and the heavier Pt isotopes are concentrated in sulphides. The shift in the Pt isotope compositions of acapulcoites and ureilites with respect to chondrites, where only a minor amount of metal-silicate differentiation can have occurred, suggests that core formation on Earth should have left the mantle extremely enriched in the heavy isotopes. The absence of such a fractionation suggests that the mantle did not acquire its Pt budget by metal-silicate equilibration in a magma ocean.

\section{Pt stable isotope evolution of Earth's mantle}

While primitive achondrites and ureilites provide a minimum constraint on the magnitude of the fractionation, they represent only the earliest stages of this process. The magnitude of $\mathrm{Pt}$ isotopic fractionation during formation of Earth's core may be expected to have been much larger (even in a magma ocean scenario), resulting in much heavier isotopic compositions in the mantle. Thus, a mechanism for shifting the isotopic composition of the fractionated mantle back to a lighter composition is required to explain the Pt isotopic composition of the present day mantle. The overlap in the composition of the mantle with chondrites is consistent with delivery of Earth's HSE budget after core formation through a chondritic late-veneer. Given the degree of mantle depletion of HSE during core formation and the amount of Pt inferred to have been added to the mantle by the late-veneer to produce the observed mantle abundances, the Pt-rich chondritic late-veneer should have ample leverage to overwhelm the isotopic composition of the mantle. To test this we performed simple mixing calculations between the late-veneer and the post-core-formation mantle (PCFM) to estimate both the resulting abundance and isotopic composition of $\mathrm{Pt}$ in the mantle (Figure 4.2). The 
Table 4.2: Model parameters used in mixing models shown in Fig. 4.2 The top table shows the partition coefficients and resulting PCFM Pt concentrations under high and low pressure and temperature core formation scenarios, and $\mathrm{Pt}$ concentrations and isotopic compositions of the reservoirs involved in the mixing calculations.

\begin{tabular}{lcc}
\hline $\begin{array}{l}\text { Core formation } \\
\text { scenario }\end{array}$ & $\mathrm{D}(\mathrm{Pt})_{\mathrm{met} / \mathrm{sil}}{ }^{a}$ & $\begin{array}{c}\text { Pt conc. } \\
\text { of PCFM } \\
(\mathrm{ppb})\end{array}$ \\
\hline $\begin{array}{l}\text { High PT } \\
\text { Low PT }\end{array}$ & $10^{4}$ & 0.144 \\
\hline & $10^{8}$ & $<1.44 \times 10^{-5}$ \\
\hline Reservoir & $\begin{array}{c}\text { Pt conc. } \\
(\mathrm{ppb})\end{array}$ & $\begin{array}{c}\delta^{198} \mathrm{Pt}^{d} \\
(\% o)\end{array}$ \\
\hline Mantle & $7.63^{b}$ & -0.27 \\
Late Veneer $(\mathrm{LV})$ & $982^{c}$ & -0.14 \\
Core material $(\mathrm{CM})$ & $5700^{c}$ & -0.14 \\
\hline
\end{tabular}

${ }^{a}$ based on high pressure and temperature (Cottrell and Walker, 2006, Holzheid et al. 2000) and low pressure and temperature (e.g., Ertel et al. 1999 partitioning experiments for Pt. ${ }^{b} \mathrm{Pt}$ concentration of PUM from Becker et al. (2006). ${ }^{c}$ based on Pt concentrations of CI chondrites (LV) and Earth's core (CM) from McDonough (2003). ${ }^{d}$ the isotopic compositions of the mantle and chondrites (LV and CM) determined in this study, where the core is assumed to have a $\sim$ chondritic isotopic composition.

calculated amount of late-veneer material required to reproduce the mantle abundance of Pt (ca. 0.5\% Earth's mass), as shown in Fig. 4.2 A, is consistent with previous estimates for the mass of the late-veneer (e.g., Chou, 1978; Holzheid et al. 2000, Dauphas and Marty, 2002). Assuming Pt abundances in the PCFM that would be consistent with core formation in a magma ocean $\left(\mathrm{D}(\mathrm{Pt})_{\mathrm{met} / \mathrm{sil}}=10^{4}\right.$; Holzheid et al., 2000, Cottrell and Walker, 2006), these calculations show that the addition of ca. $0.5 \%$ of Earth's mass of chondritic material would be sufficient to overprint the Pt isotopic composition of the mantle if the $\delta^{198} \mathrm{Pt}$ of the PCFM was ca. $\leq 2.0 \%$ (Fig. $4.2 \mathrm{~B}$ ). The amount of late-veneer material required can be reduced if higher concentrations of Pt for chondrites (e.g., ca. 1100-1500 ppb; Walker et al., 2002; Horan et al. 2003 ) or lower partitioning coefficients for Pt are used (Fig. 4.2B).

We note, however, that the isotopic compositions of the mantle and chondrites are not identical. While the Pt isotopic composition of the mantle over- 


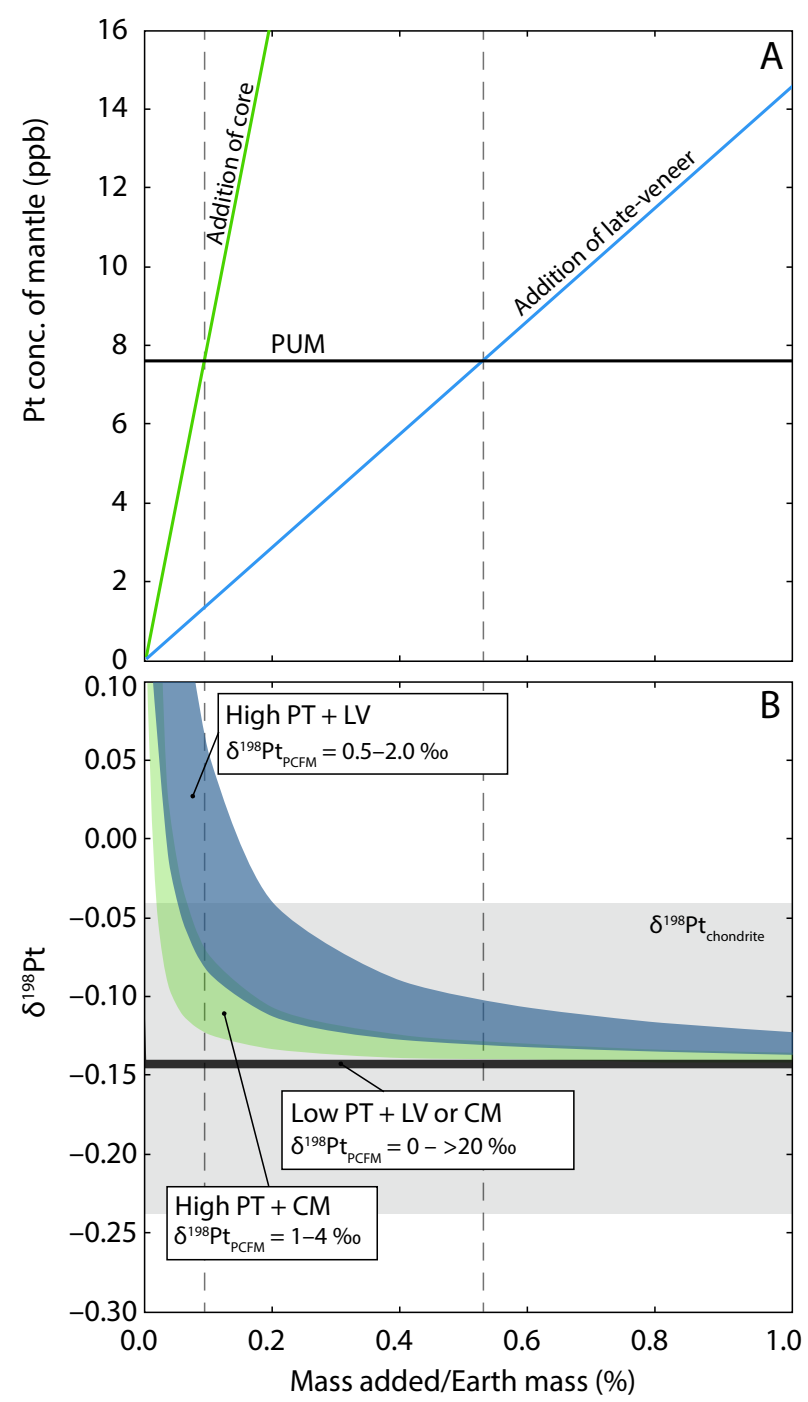

Figure 4.2: Modelling the effect of the late-veneer (LV) or addition of core material (CM) on the abundance and isotopic composition of $\mathrm{Pt}$ in the mantle. Parameters used in the models are given in Table 4.2 (A) The calculated concentration of Pt in the PUM from the addition of LV or CM to the post-core-formation mantle (PCFM). The horizontal line represents the Pt concentration of PUM (7.6 ppb; Becker et al., 2006). Vertical dashed lines mark the intercepts between the two mixing lines and the PUM, indicating the amount of addition required to reproduce the Pt abundance of PUM, which are consistent with previous estimates for the mass of the late-veneer (e.g., Chou 1978 Holzheid et al. 2000: Dauphas and Marty 2002). The change in the mixing lines resulting from different Pt abundances in the PCFM with different conditions of core formation (i.e., $\mathrm{D}(\mathrm{Pt})_{\mathrm{met}} / \mathrm{sil}$ in the range 104-1012; Ertel et al. 1999 Holzheid et al. 2000, Cottrell and Walker 2006) is negligible on this scale. (B) The effect on the Pt isotopic composition of the mantle from mixing different proportions of late-veneer or core material with the PCFM. The magnitude of Pt stable isotopic fractionation in the PCFM (i.e., prior to any addition) is also indicated in the boxes. The concentration of Pt in the PCFM changes under the different core formation scenarios as shown in Table 4.2 The grey shaded box represents the compositional range of chondrites. 
laps with chondrites, the compositions of mantle samples extend to more negative $\delta^{198} \mathrm{Pt}$ values than those observed in chondrites by ca. $0.10 \%$ (Fig. 4.1). A Student's t-test comparing the mean of the terrestrial mantle samples suggests that this difference is statistically significant at the $99.55 \%$ confidence level $\left(\mathrm{t}_{\text {calculated }}=3.37, \mathrm{df}=14\right)$. Possible reasons for this offset could include:

(1) The chondrite samples used in this study may have not yet sampled the full compositional range of chondrites. As more chondrite samples are measured it may be found that the range of chondritic compositions entirely overlaps with those of Earth's mantle. The isotopic heterogeneity of the mantle may also provide support for the late-veneer, as a magma ocean scenario would be expected to have allowed Pt to isotopically equilibrate.

(2) The composition of the mantle may not yet be sufficiently well constrained. Additional samples may show that the average mantle composition is more chondritic in nature, and that the variations observed thus far may represent other within-mantle processes (e.g., melt extraction, mantle metasomatism), which have also been suggested to explain non-chondritic PGE abundances in the mantle (e.g., Handler and Bennett, 1999).

(3) The late-veneer material may not have been chondritic in composition, although this seems unlikely given the numerous lines of evidence that have been used to argue for a chondritic late-veneer (e.g., Chou, 1978; Morgan. 1986; Meisel et al., 1996; Shirey and Walker, 1998, Morbidelli et al., 2000. Holzheid et al., 2000; Walker et al., 2001; Drake and Righter, 2002; Dauphas and Marty, 2002; Righter, 2003; Fischer-Gödde et al., 2011; Dale et al., 2012).

(4) HSE abundances in the mantle may not be derived from a late-veneer or metal-silicate equilibration in a magma ocean, and another scenario is responsible. Another hypothesis that has received some attention is that chemical or physical exchange could occur between the liquid outer core and the lower mantle (Poirier and le Mouël, 1992; Walker et al., 1995; Brandon and Walker, 2005), potentially resulting in transport of HSE back into the mantle (Walker et al., 1995, Brandon et al., 1998, Snow and Schmidt, 1998, Brandon and Walker, 2005). Greater core-mantle exchange during the Archean due 
to more vigorous convection and mixing given the greater heat flux at the time (Richter, 1985; Nisbet et al., 1993) could provide an alternative explanation for the observed increase in mantle HSE abundances during that period, which have been interpreted to reflect progressive mixing of late veneer material into the mantle (e.g., Maier et al., 2009). This model was also tested in the mixing calculations presented in Fig. 4.2. The calculations show that the addition of $<0.1 \%$ of Earth's mass of core material could explain the mantle abundance of $\mathrm{Pt}$ (Fig. 4.2). The addition of core material to the mantle would also be expected to impart a chondritic isotopic signature, given that core incorporated $>99.99 \%$ of HSE, although some mass-dependent fractionation could potentially occur during exchange at the core-mantle boundary, or during fractionation between solid inner core and liquid outer core over time, which could then be transferred to the mantle. As shown in Fig. 4.2 , the addition of ca. $0.1 \%$ of Earth's mass of core material to the mantle could overprint a PCFM composition of $\delta^{198} \mathrm{Pt}=\leq 4.0 \%$. However, mechanisms for mass transfer or chemical exchange between the outer core are complex (Brandon and Walker, 2005), and have a range of consequences beyond what is considered here. The transfer of the relatively large amount of material between the core and mantle required by the calculations in Fig. 4.2 may be implausible.

Further investigation is required to determine whether points 1-3 satisfactorily explain the differences in the Pt stable isotope composition of the mantle and chondrites. The wider compositional range of $\mathrm{Pt}$ stable isotopes in the mantle may reflect within-mantle processes, such as melt-extraction or mantle metasomatism, which could be easily reconciled with a chondritic late-veneer. Critical gaps in our understanding of $\mathrm{Pt}$ isotopic fractionations during these processes may be filled by combining isotopic measurements with experimental petrology and/or thermodynamic modelling, providing a framework to interpret and constrain isotopic results. However, these are not currently available. Based on the available data, the Pt stable isotopic measurements presented here are most consistent with addition of a chon- 


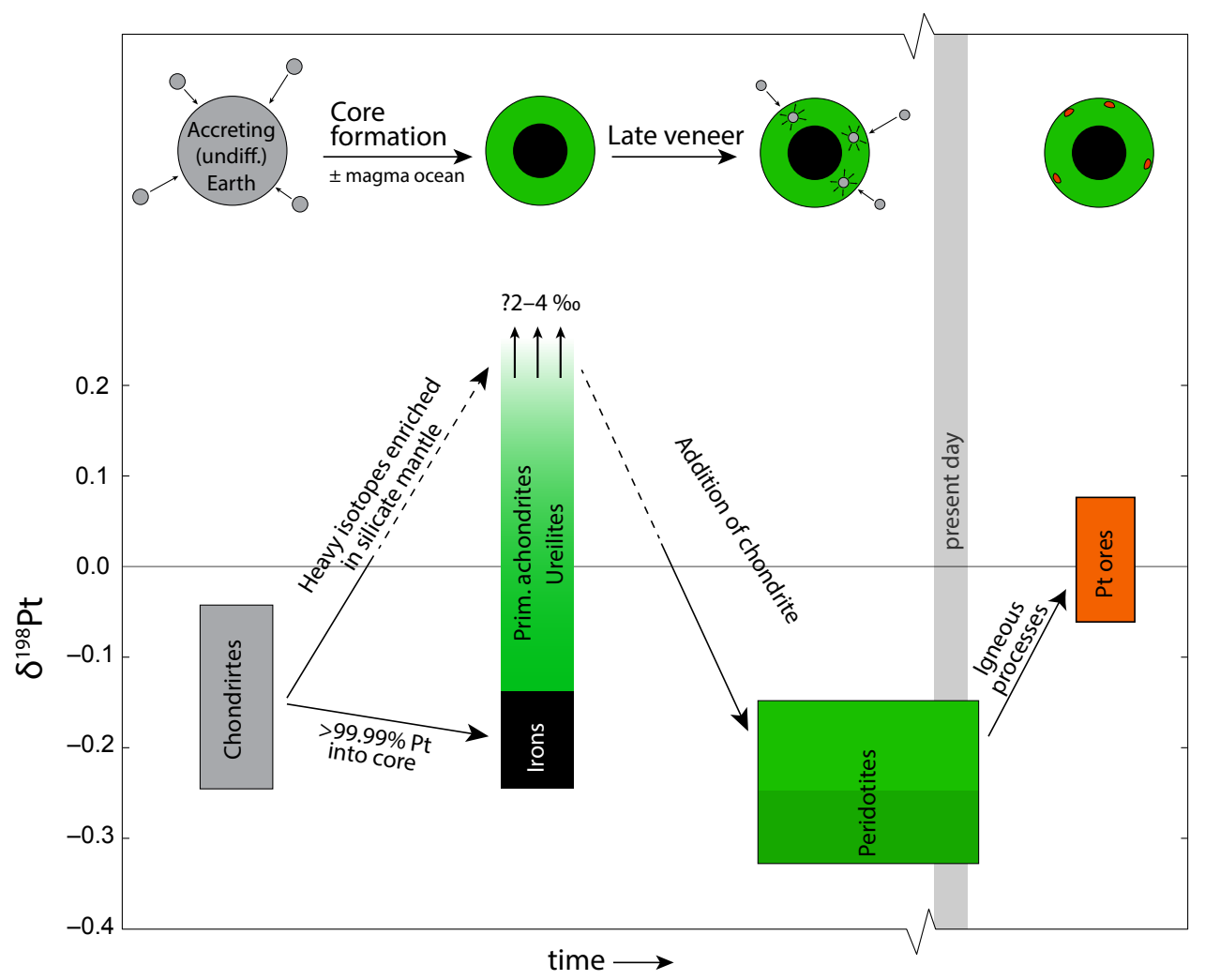

Figure 4.3: Schematic cartoon of the hypothetical Pt stable isotope evolution of Earth's core and mantle based on measurements of terrestrial samples and meteorites in this study. The compositional ranges in the coloured boxes are constrained by the samples indicated inside the box, and link to the colours in the planet cartoons.

dritic late-veneer, and our currently preferred model for the Pt stable isotope evolution of Earth is presented in Fig. 4.3. In this model, the undifferentiated Earth had a chondritic starting composition. Metal-silicate differentiation during core formation partitioned Pt (and HSE) into the core and caused the concomitant depletion of $\mathrm{Pt}$ in the mantle, and also isotopically fractionated $\mathrm{Pt}$ in the PCFM towards a heavy Pt isotopic composition, as is suggested by the Pt stable isotopic composition of primitive achondrites and ureilites (Table 4.1) Fig. 4.1). The addition of the late-veneer then overprinted the Pt stable isotopic signature of core formation in the PCFM with a chondritic composition. The calculations in Fig. 4.2 show that the addition of 
the generally accepted mass (0.5\% of Earth) of late-veneer material would be sufficient to overprint a PCFM Pt isotopic signature of up to $2.0 \%$ in a magma ocean scenario for core formation, or $\geq 20.0 \%$ if core formation occurred at lower pressures and temperatures.

\subsection{Conclusions}

We have determined the mass-dependent Pt stable isotopic fractionations in meteorites and terrestrial samples. Pt stable isotope data from these samples have shown that:

(1) The Pt stable isotopic composition of Earth's mantle overlaps that of ordinary, enstatite and carbonaceous chondrites.

(2) Partitioning of Pt during metal-silicate differentiation appears to preferentially incorporate lighter isotopes into the metal phase, with heavier isotopes retained in the silicate phase. Primitive achondrites and ureilites, which represent the very earliest stages of this process, show fractionations that are 0.06- $0.15 \%$ o heavier than chondrites, which suggests that core formation on Earth should have produced large (per mil scale) Pt isotopic fractionations in the mantle. The overlap between the mantle composition and chondrites suggests that the isotopic signature of this process has been overprinted in Earth's mantle.

(3) The overlap between the Pt isotopic compositions of Earth's mantle and chondrites is consistent with the addition of a late-veneer of $0.5 \%$ of Earth's mass of chondritic material to Earth after core formation. Calculations show that this mass of chondritic material is sufficient to overprint large $\mathrm{Pt}$ isotopic fractionations in the post-core-formation mantle, even if core formation took place in a magma ocean prior to delivery of the lateveneer. A small but statistically significant difference between the mantle and chondrite compositions is interpreted to reflect within-mantle processes. 


\section{Acknowledgements}

This research was supported by a Royal Society of New Zealand Marsden Grant to MH and JB. JC is funded by a VUW PhD scholarship. The Centre for Star and Planet Formation is funded by the Danish National Research Foundation and the University of Copenhagen's Programme of Excellence. 


\section{Chapter 5}

\section{Conclusions}

Non-traditional stable isotope systems are playing an increasingly important role in improving our understanding of diverse natural processes. The Pt stable isotope system represents a potentially powerful new geochemical tracer, with a range of possible applications. The geochemical behaviour of Pt makes this isotope system uniquely suited to tracing certain processes, such as the accretion and differentiation of Earth. Two key analytical challenges were identified that had to be overcome in order to investigate Pt stable isotope variations in natural samples: (1) the development of new analytical techniques for accurate measurement of Pt stable isotope ratios, with sufficient precision to resolve the relatively small fractionations expected in nature; and (2) reproducible methods had to be developed for chemical separation of Pt from geological samples, with sufficiently high recovery and purity of $\mathrm{Pt}$ to enable precise and accurate $\mathrm{Pt}$ stable isotope measurements to be made. The development of these techniques comprised the first major objective of this thesis.

The second major objective of this research was to apply these techniques to measuring $\mathrm{Pt}$ stable isotope ratios in a range of natural samples, with four key sub-goals: (1) to constrain the Pt isotopic compositions of the major terrestrial reservoirs, and the magnitude of Pt stable isotopic fractionations in high-temperature Earth processes; (2) to investigate the range of Pt stable 
isotopic variations amongst primitive meteorites (i.e., chondrites) to constrain the composition of the building blocks of the terrestrial planets; (3) to measure Pt stable isotopic compositions of primitive achondrites, achondrites and iron meteorites to constrain the magnitude of isotopic fractionations associated with core formation in differentiated planetary bodies; and (4) to use these data to test models of Earth's formation and differentiation and to place new constraints on these processes.

The conclusions of this thesis are presented below in the context of these two major objectives and their sub-goals.

\subsection{Techniques for platinum stable isotope measurements}

Based on the geochemistry of Pt, as outlined in Chapter 1, potential applications for the Pt stable isotope system can be identified in several important areas of Earth science (e.g., planetary geology, ocean geochemistry). In addition, Pt has a wide range of uses outside of the Earth sciences (e.g., medicine, environmental science; Chapter 2), where further applications for this stable isotope tracer might be found. Despite the potential of this tool, the Pt stable isotope system and its applications to natural samples remained unexplored prior to this thesis research. This may be partly due to the difficulties in making stable isotope measurements of heavy elements at sufficient precision to resolve small natural isotopic fractionations, and the required instruments and techniques for making such measurements have only been developed relatively recently. Two major analytical challenges for measuring Pt stable isotope ratios in natural samples were identified above, and the conclusions of this thesis are discussed below under headings relating to these challenges. 


\subsubsection{Precise and accurate measurement of platinum stable isotope ratios}

The development of techniques for measurement of Pt stable isotope ratios was presented in Chapter 2. This chapter described the development of two Pt double-spikes (DS) for the correction of MC-ICPMS data: a ${ }^{192} \mathrm{Pt}-{ }^{198} \mathrm{Pt}$ DS and a ${ }^{196} \mathrm{Pt}-{ }^{198} \mathrm{Pt}$ DS. The reporting of $\mathrm{Pt}$ stable isotope variations as $\mu^{198} \mathrm{Pt}$ was first defined in this chapter, representing parts per million variations in the ${ }^{198} \mathrm{Pt} /{ }^{194} \mathrm{Pt}$ ratio from the same ratio in the IRMM-010 $\mathrm{Pt}$ isotope standard.

Repeated measurements of the IRMM-010 Pt standard were made in two different laboratories. These measurements, each consuming ca. 40-85 ng of $\mathrm{Pt}$, show that a long-term external reproducibility for $\mu^{198} \mathrm{Pt}$ of $\leq 40 \mathrm{ppm}$ (2 sd; i.e., $\leq 10 \mathrm{ppm} \mathrm{amu}^{-1}$ ) can be obtained on Pt stable isotope ratios with either double-spike. Using this technique, the isotopic compositions of several Pt elemental solution standards were measured, revealing resolvable difference in $\mathrm{Pt}$ stable isotope ratios between the elemental standards and the IRMM-010 standard, and suggesting that natural Pt stable isotope fractionations exist that are larger than the reproducibility of this technique.

Elemental doping tests were conducted to reveal the sensitivity of $\mathrm{Pt}$ stable isotope measurements by DS MC-ICPMS to minor amounts of matrix that may be retained after chemical separation of Pt. These tests revealed that DS corrected Pt stable isotope ratios with either DS are insensitive to the presence of relatively high (up to 10\%) levels of matrix elements, with one key exception. Measurements using the ${ }^{192} \mathrm{Pt}-{ }^{198} \mathrm{Pt}$ double-spike are catastrophically affected by minor amounts of Os remaining in the sample, due to an isobaric interference on ${ }^{192} \mathrm{Pt}$ from ${ }^{192} \mathrm{Os}$. The ${ }^{196} \mathrm{Pt}-{ }^{198} \mathrm{Pt}$ DS does not use ${ }^{192} \mathrm{Pt}$ in the DS calculations, and is thus unaffected by Os contamination. Due to the sensitivity of the ${ }^{192} \mathrm{Pt}-{ }^{198} \mathrm{Pt}$ to small amounts of Os contamination, as well as the greater potential for disturbance of $\mathrm{Pt}$ stable isotope ratios by cosmogenic effects using that DS, the ${ }^{196} \mathrm{Pt}-{ }^{198} \mathrm{Pt}$ DS 
was selected as the preferred DS in measurements of natural samples.

Finally, as part of the work described in Chapter 2, the natural isotopic composition of $\mathrm{Pt}$, which is defined by the composition of the IRMM-010 standard (Berglund and Wieser, 2011), was redetermined by MC-ICPMS using external element $(\mathrm{Pb})$ doping to correct for instrumental mass bias. Relative Pt isotope differences of up to $10 \%$ from the reference values were identified for this standard. The new isotopic composition of the IRMM010 standard $\left({ }^{190} \mathrm{Pt}=0.01289 \%,{ }^{192} \mathrm{Pt}=0.7938 \%,{ }^{194} \mathrm{Pt}=32.81 \%,{ }^{195} \mathrm{Pt}=\right.$ $33.79 \%,{ }^{196} \mathrm{Pt}=25.29 \%$ and ${ }^{198} \mathrm{Pt}=7.308 \%$ ) results in a redefined $\mathrm{Pt}$ atomic weight of $195.08395 \pm 0.00068$.

\subsubsection{Purification of platinum from natural samples}

Procedures for preparation of natural samples for Pt stable isotope analysis by double-spike MC-ICPMS were presented in Chapter 3. This chapter described methods for digestion of samples using a nickel-sulphide fire assay technique, which was applied to all terrestrial samples presented in Chapter 3 and Chapter 4 , as well as all stony meteorite (i.e., chondrite and achondrite) samples presented in Chapter 4. An additional procedure for acid digestion of iron meteorite samples was described in Chapter 4. Samples were generally double-spiked prior to digestion, and the technique should thus correct for any mass-dependent $\mathrm{Pt}$ isotopic fractionation in the samples that might occur during sample digestion or Pt chemical purification.

Purification of Pt from samples was conducted by anion-exchange chromatography. Major cationic constituents of the samples are not retained on the anion exchange resin and pass directly through the column into the effluent upon sample loading. Weakly retained transition metals and other platinum group elements (excluding platinum) are eluted using $\mathrm{HCl}$ and $\mathrm{HNO}_{3}$ acids of increasing concentration. $\mathrm{Pt}$ is finally recovered from the anion exchange resin in concentrated $\mathrm{HNO}_{3}$. The separation method was calibrated using a range of synthetic rock matrices, and the results showed that $\mathrm{Pt}$ yields $\geq 90 \%$ with purity $\geq 95 \%$ were routinely achievable using this tech- 
nique. The element doping tests presented in Chapter 2 demonstrated that this level of separation is sufficient for accurate isotope ratio measurements by DS MC-ICPMS.

The application of these techniques to terrestrial standard reference materials was also presented in Chapter 3. The results of these measurements showed readily resolvable fractionations between mantle, igneous and $\mathrm{Pt}$ ore samples. The reproducibility of these methods as applied to natural samples was estimated by measuring repeated digestions of a terrestrial standard, and was found to be ca. $\pm 75 \mathrm{ppm}(2 \mathrm{sd})$ on $\mu^{198} \mathrm{Pt}$. As a result, Pt stable isotope results were subsquently reported in delta notation, $\delta^{198} \mathrm{Pt}$, with units of $\%$ o (i.e., parts per thousand). While it may be possible to improve these uncertainties in the future, the variations observed between natural samples suggest that this level of uncertainty is sufficient to resolve natural fractionations in a range of geological samples.

\subsection{Platinum stable isotopes in terrestrial and meteorite samples and implications for accretion and differentiation models}

\subsubsection{Platinum stable isotopes in terrestrial samples}

Platinum stable isotope compositions of 11 international geological standard reference materials were presented in Chapter 3 . These standards comprised PGE ores, mantle rocks and igneous rocks, with Pt concentrations in these samples ranging from ca. $5 \mathrm{ng} \mathrm{g}^{-1}$ to $4 \mathrm{\mu g} \mathrm{g}^{-1}$. Pt stable isotope data for the full set of reference materials showed a range of $\delta^{198} \mathrm{Pt}$ values, with offsets of up to $0.40 \%$ o from the standard that are readily resolved with this technique. The peridotite standards showed the lightest Pt isotopic compositions, with $\delta^{198} \mathrm{Pt}$ of ca. $-0.33 \%$. Most igneous samples have Pt stable isotopic compositions intermediate between the mantle peridotites and $\mathrm{Pt}$ ore samples, 
which have $\delta^{198} \mathrm{Pt}$ values of ca. $0.00 \pm 0.06 \%$. The similarity between $\mathrm{Pt}$ ore samples and the IRMM-010 Pt isotope standard and Pt elemental solution standards is consistent with these standards being prepared from $\mathrm{Pt}$ ores. The resolution of natural isotopic fractionations between the terrestrial standards demonstrates the potential of the Pt isotope system as a tracer in high-temperature geochemical systems.

Pt stable isotope data for two additional mantle peridotite samples were presented in Chapter 4. Combined with the mantle peridotite samples from Chapter 3 , these data provide a first constraint on the Pt stable isotopic composition of the mantle; $\delta^{198} \mathrm{Pt}_{\text {mantle }}=-0.27 \pm 0.15 \%$ o $(2 \mathrm{sd})$. The relatively broad range of compositions observed in mantle samples suggests that the mantle is isotopically heterogeneous for Pt. Possible explanations for this variability may include melt extraction or mantle metasomatism, which have also been proposed to explain heterogeneities in PGE abundances between mantle samples.

\subsubsection{Platinum stable isotopes in meteorites}

Platinum stable isotope compositions analysed in ordinary, enstatite and carbonaceous chondrites, primitive achondrites, achondrites and iron meteorites were presented in Chapter 4. The mean Pt stable isotopic compositions from ordinary, enstatite and carbonaceous chondrites were found to be similar, although carbonaceous chondrites showed greater variability than the other chondrite groups. The variations amongst carbonaceous chondrites could potentially reflect pre-solar Pt stable isotopic variations recorded in their different components, which requires further investigation. The Pt stable isotopic composition of chondrites as a group was found to be $\delta^{198} \mathrm{Pt}_{\text {chondrite }}=-0.14 \pm 0.10 \%$ o $(2 \mathrm{sd})$. The composition of chondrites provides two important constraints for accretion and differentiation models: the composition of the undifferentiated Earth, which provides a reference against which isotopic fractionations in the mantle can be measured, and the isotopic composition of the putative late-veneer material. 
Iron meteorites were found to overlap in Pt isotope compositions with chondrites. As the Pt budget of the asteroidal parent bodies that these meteorites sample is expected to have been quantitatively partitioned into their cores, the Pt isotopic compositions of iron meteorites are thus interpreted to represent that of the precursor material from which they formed, which is likely to have been chondrite-like.

Primitive achondrites and ureilites samples have Pt isotopic compositions that are $0.06-0.15 \%$ o heavier than chondrites. These results suggest the partitioning of Pt during metal-silicate differentiation preferentially incorporates lighter isotopes into the metal phase, with heavier isotopes being retained in the silicate phase.

\subsubsection{Platinum stable isotope implications for accre- tion and differentiation models}

Based on the fractionation observed in primitive achondrites and ureilites, the Pt stable isotopic composition of Earth's mantle after core formation is expected to have been fractionated towards heavier compositions. The lack of such fractionated compositions in samples of Earth's mantle suggests that this fractionation has been overprinted by subsequent processes. Given the overlap between the $\mathrm{Pt}$ isotope compositions of the mantle and chondrites, the most likely mechanism for overprinting this extremely fractionated composition in the mantle is the addition of a chondritic late-veneer. Models of mixing between late-veneer material and the mantle composition after core formation show that if the core formed under a high pressure and temperature (i.e., magma ocean) regime, the addition of $0.5 \%$ Earth's mass of late-veneer material could have overprinted Pt stable isotopic fractionations in the postcore-formation mantle of up to $2.0 \%$. If Earth's core formed under lower pressure and temperature conditions, the late-veneer could overprint isotopic compositions in the post-core-formation mantle of $\geq 20 \%$. Therefore, the Pt stable isotope data is consistent with a late-veneer of $0.5 \%$ Earth's mass 
of chondritic material that was added after core formation.

\subsection{Suggestions for future work}

Based on the findings of this thesis, a range of possible areas of future research have been identified. It is hoped that additional investigations utilising the methods developed in this thesis will be forthcoming, to substantiate and further the results of the research presented here, as well as to explore other areas where this tracer might be applied.

\section{Further investigations of planetary differentiation}

The data for terrestrial and meteorite samples analysed in this thesis represent a very preliminary attempt to constrain the compositions of these different groups of samples. Many more analyses of these different groups of samples are required to adequately constrain the Pt stable isotopic compositions of these different reservoirs and to fully understand the processes causing the isotopic fractionations. Therefore, one aspect of further research is simply to extend the data set for the samples analysed in this study.

Additionally, other groups of meteorites could be analysed for Pt stable isotopes and provide further constraints for models of terrestrial planet accretion and differentiation. As described in this thesis, ureilites exhibit features of both primitive and differentiated achondrites, and are thought to represent the mantle of an asteroid that only partially melted. Ureilite meteorites show a range of siderophile element depletions (Janssens et al., 1987. Goodrich et al., 1987; Rankenburg et al., 2008), which may relate to different degrees of metal-silicate differentiation on the ureilite parent body. If so, ureilite samples with different siderophile element abundances may exhibit distinct Pt stable isotope compositions, which would provide additional constraints for interpretation of Pt stable isotope data. Other differentiated achondrite samples could yield further constraints on the magnitude of stable 
isotope fractionation during planetary differentiation. Meteorites sampling the silicate portions of planetesimals that experienced complete melting and differentiation (e.g., diogenites, silicates from pallasites) should be isotopically heavier than the primitive achondrite and ureilite samples presented in this thesis. However, the concentrations of Pt in such samples are likely to be relatively low (i.e., $<1 \mathrm{ng} \mathrm{g}^{-1}$ ), and thus large samples would be required for $(>10 \mathrm{~g})$ Pt stable isotope analysis.

Finally, the most interesting possible addition to the sample set for investigating planetary differentiation would be to analyse samples from the Moon or Mars. Both of these represent large differentiated planetary bodies, which formed and differentiated under significantly different conditions and on very different timescales to each other and to Earth. Additionally, these bodies lack the dynamic mantle that exists on Earth, and thus delivery of the late-veneer material may not have overprinted the Pt stable isotopic signature of core formation in their mantles. However, due to the size of samples required for Pt stable isotope analysis and the difficulty in obtaining samples from these bodies, Martian and Lunar samples for Pt stable isotopic analysis may not be forthcoming.

\section{Nucleosynthetic inputs of Pt in the solar system}

As described in this thesis, Pt stable isotopic variations amongst carbonaceous chondrites were greater than those amongst ordinary and enstatite chondrite groups. Given the small number of enstatite and ordinary chondrite samples, is not yet possible to say if this greater variability is statistically meaningful. However, one possible explanation for these variations could be that they reflect different Pt stable isotopic compositions recorded in the different components of carbonaceous chondrites (e.g., chondrules, matrix, refractory inclusions). Such variations could reflect pre-solar differences in Pt stable isotope ratios, which could be studied by measuring Pt stable isotopes in the different components of chondrites, although these measurements would need to be made using a standard-sample-standard bracketing 
approach, as opposed to the double-spike method used in this thesis, and potential mass-dependent fractionations during sample processing (which are normally accounted for by the double-spike correction) will need to be addressed. If the fractionations in these components are mass-dependent processes, they may be related to processes such as evaporation and condensation in the solar nebula. Mass-independent fractionations could arise due to different nucleosynthetic inputs into the early solar system, and thus Pt stable isotope measurements could provide constraints on the astrophysical setting of solar system formation.

\section{Experimental and theoretical validation of isotopic frac- tionation measurements}

Important gaps in our understanding of Pt stable isotope systematics could be filled by the addition of quantitative experimental or theoretical constraints on isotopic fractionations under known conditions of pressure, temperature and oxygen fugacity. High pressure and temperature experiments have already played a key role in our understanding of metal-silicate partitioning of Pt. Performing similar experiments where samples are doped with a known composition of $\mathrm{Pt}$ would allow stable isotopic fractionations under different experimental conditions to be quantified. The difficulty in such experiments would be in producing enough material for Pt stable isotope measurements, as the $\mathrm{Pt}$ concentrations in the silicate products of these experiments would be extremely low, and isotopic fractionations in the metal products may be vanishingly small. Thermodynamic modelling may provide an alternative method for constraining the magnitude and direction of $\mathrm{Pt}$ stable isotopic fractionations under different conditions. Similar models have been developed for a range of other isotope systems and have provided important constraints for the interpretation of isotopic data (Schauble et al. 2007; Georg et al., 2007; Shahar et al., 2009; Ziegler et al., 2010; Moynier et al., 2011). 


\section{Combined References}

JCGM 100:2008 GUM 1995 with minor corrections, Evaluation of Measurement Data-Guide to the Expression of Uncertainty in Measurements. Joint Committee for Guides in Metrology (BIPM, IEC, IFCC, ILAC, ISO, IUPAC, IUPAP and OIML). http://www.iso.org/sites/JCGM/GUM-introduction.htm.

Abrams, M. J. and Murrer, B. A., 1993. Metal compounds in therapy and diagnosis. Science, 261, 725-730. doi: 10.1126/science.8102010.

Alard, O., Griffin, W. L., Lorand, J. P., Jackson, S. E., and O’Reilly, S. Y., 2000. Nonchondritic distribution of the highly siderophile elements in mantle sulphides. Nature, 407, 891-894. doi: 10.1038/35038049.

Albarède, F., 2003. Geochemistry. Cambridge, UK: Cambridge University Press.

Albarède, F., 2004. The stable isotope geochemistry of copper and zinc. Reviews in Mineralogy and Geochemistry, 55, 409-427. doi: 10.2138/gsrmg.55.1.409.

Albarède, F., 2009. Volatile accretion history of the terrestrial planets and dynamic implications. Nature, 461, 1227-1233. doi: 10.1038/nature08477.

Albarède, F. and Beard, B., 2004. Analytical methods for non-traditional isotopes. Reviews in Mineralogy and Geochemistry, 55, 113-152. doi: 10.2138/gsrmg.55.1.113.

Allègre, C. J., Poirier, J.-P., Humler, E., and Hofmann, A. W., 1995. The chemical composition of the Earth. Earth and Planetary Science Letters, 134, 515-526.

Alvarez, L. W., Alvarez, W., Asaro, F., and Michel, H. V., 1980. Extraterrestrial cause for the Cretaceous-Tertiary extinction. Science, 208, 1095-1108. doi: 10.1126/science. 208.4448.1095.

Amelin, Y., Krot, A. N., Hutcheon, I. D., and Ulyanov, A. A., 2002. Lead isotopic ages of chondrules and calcium-aluminum-rich inclusions. Science, 297, 1678-1683. doi: 10.1126/science.1073950.

Anbar, A. D., Knab, K. A., and Barling, J., 2001. Precise determination of mass-dependent 
variations in the isotopic composition of molybdenum using MC-ICPMS. Analytical Chemistry, 73, 1425-1431. doi: 10.1021/ac000829w.

Anbar, A. D., 2004. Molybdenum stable isotopes: Observations, interpretations and directions. Reviews in Mineralogy and Geochemistry, 55, 429-454. doi: 10.2138/gsrmg. 55.1.429.

Anbar, A. D. and Rouxel, O., 2007. Metal stable isotopes in paleoceanography. Annual Review of Earth and Planetary Sciences, 35, 717-746. doi: 10.1146/annurev.earth.34. 031405.125029 .

Anders, E., 1962. Meteorite ages. Reviews of Modern Physics, 34, 287-325. doi: 10.1103/ RevModPhys.34.287.

Anders, E. and Grevesse, N., 1989. Abundances of the elements: Meteoritic and solar. Geochimica et Cosmochimica Acta, 53, 197-214. doi: 10.1016/0016-7037(89)90286-X.

Anthony, J., Bideaux, R., Bladh, K., and Nichols, M., 2004. Handbook of Mineralogy. Mineralogical Society of America.

Armytage, R., Georg, R., Savage, P., Williams, H., and Halliday, A., 2011. Silicon isotopes in meteorites and planetary core formation. Geochimica et Cosmochimica Acta, 75, 3662-3676. doi: 10.1016/j.gca.2011.03.044.

Armytage, R., Georg, R., Williams, H., and Halliday, A., 2012. Silicon isotopes in lunar rocks: Implications for the Moon's formation and the early history of the Earth. Geochimica et Cosmochimica Acta, 77, 504-514. doi: 10.1016/j.gca.2011.10.032.

Arnold, T., Schönbächler, M., Rehkämper, M., Dong, S., Zhao, F.-J., Kirk, G. J. D., Coles, B. J., and Weiss, D. J., 2010. Measurement of zinc stable isotope ratios in biogeochemical matrices by double-spike MC-ICPMS and determination of the isotope ratio pool available for plants from soil. Analytical and Bioanalytical Chemistry, 398, 3115-3125. doi: 10.1007/s00216-010-4231-5.

Audi, G., Bersillon, O., Blachot, J., and Wapstra, A., 2003. The nubase evaluation of nuclear and decay properties. Nuclear Physics A, 729, 3-128. doi: 10.1016/j.nuclphysa. 2003.11.001.

Baker, J., Peate, D., Waight, T., and Meyzen, C., 2004. Pb isotopic analysis of standards and samples using a ${ }^{207} \mathrm{~Pb}^{204} \mathrm{~Pb}$ double spike and thallium to correct for mass bias with a double-focusing MC-ICP-MS. Chemical Geology, 211, 275-303. doi: 10.1016/j. chemgeo.2004.06.030.

Ballhaus, C. G. and Stumpfl, E. F., 1986. Sulfide and platinum mineralization in the Merensky Reef: evidence from hydrous silicates and fluid inclusions. Contributions to Mineralogy and Petrology, 94, 193-204. doi: 10.1007/BF00592936. 
Banakar, V., Hein, J., Rajani, R., and Chodankar, A., 2007. Platinum group elements and gold in ferromanganese crusts from Afanasiy-Nikitin seamount, equatorial Indian Ocean: Sources and fractionation. Journal of Earth System Science, 116, 3-13. doi: 10.1007/s12040-007-0002-x.

Bannister, F. A., 1932. Determination of minerals in platinum concentrates from the Transvaal by X-ray methods. Mineralogical Magazine, 23, 188-206. doi: 10.1180/ minmag.1932.023.138.05.

Barkov, A. Y., Laflamme, J. H. G., Cabri, L. J., and Martin, R. F., 2002. Platinumgroup minerals from the Wellgreen Ni-Cu-PGE deposit, Yukon, Canada. The Canadian Mineralogist, 40, 651-669. doi: 10.2113/gscanmin.40.2.651.

Barnes, S.-J. and Picard, C., 1993. The behaviour of platinum-group elements during partial melting, crystal fractionation, and sulphide segregation: An example from the Cape Smith Fold Belt, northern Quebec. Geochimica et Cosmochimica Acta, 57, 79-87. doi: 10.1016/0016-7037(93)90470-H.

Barnes, S.-J., Naldrett, A., and Gorton, M., 1985. The origin of the fractionation of platinum-group elements in terrestrial magmas. Chemical Geology, 53, 303-323. doi: 10.1016/0009-2541(85)90076-2.

Beard, B. L. and Johnson, C. M., 1999. High precision iron isotope measurements of terrestrial and lunar materials. Geochimica et Cosmochimica Acta, 63, 1653-1660. doi: 10.1016/S0016-7037(99)00089-7.

Beard, B. L. and Johnson, C. M., 2004. Fe isotope variations in the modern and ancient Earth and other planetary bodies. Reviews in Mineralogy and Geochemistry, 55, 319357. doi: 10.2138/gsrmg.55.1.319.

Beard, B. L., Johnson, C. M., Cox, L., Sun, H., Nealson, K. H., and Aguilar, C., 1999. Iron isotope biosignatures. Science, 285, 1889-1892. doi: 10.1126/science.285.5435.1889.

Beard, B. L., Johnson, C. M., Skulan, J. L., Nealson, K. H., Cox, L., and Sun, H., 2003. Application of Fe isotopes to tracing the geochemical and biological cycling of Fe. Chemical Geology, 195, 87-117. doi: 10.1016/S0009-2541(02)00390-X.

Becker, H., Horan, M., Walker, R., Gao, S., Lorand, J.-P., and Rudnick, R., 2006. Highly siderophile element composition of the Earth's primitive upper mantle: Constraints from new data on peridotite massifs and xenoliths. Geochimica et Cosmochimica Acta, 70, 4528-4550. doi: 10.1016/j.gca.2006.06.004.

Belshaw, N. S., Zhu, X. K., Guo, Y., and O'Nions, R. K., 2000. High precision measurement of iron isotopes by plasma source mass spectrometry. International Journal of Mass Spectrometry, 197, 191-195. 
Belshaw, N., Freedman, P., O’Nions, R., Frank, M., and Guo, Y., 1998. A new variable dispersion double-focusing plasma mass spectrometer with performance illustrated for $\mathrm{Pb}$ isotopes. International Journal of Mass Spectrometry, 181, 51-58. doi: 10.1016/ S1387-3806(98)14150-7.

Benedix, G. K., McCoy, T. J., Keil, K., Bogard, D. D., and Garrison, D. H., 1998. A petrologic and isotopic study of winonaites: Evidence for early partial melting, brecciation, and metamorphism. Geochimica et Cosmochimica Acta, 62, 2535-2553.

Benedix, G. K., McCoy, T. J., Keil, K., and Love, S. G., 2000. A petrologic study of the IAB iron meteorites: Constraints on the formation of the IAB-Winonaite parent body. Meteoritics \& Planetary Science, 35, 1127-1141.

Bennett, V., 2003. Compositional evolution of the mantle. In Treatise on Geochemistry, Heinrich D. Holland and Karl K. Turekian (Eds.) 493-519. Pergamon, Oxford.

Benz, W., Slattery, W. L., and Cameron, A. G. W., 1987. The origin of the Moon and the single-impact hypothesis, II. Icarus, 71, 30-45.

Berglund, M. and Wieser, M. E., 2011. Isotopic compositions of the elements 2009 (IUPAC technical report). Pure and Applied Chemistry, 83, 397-410. doi: 10.1351/ PAC-REP-10-06-02.

Birch, F., 1964. Density and composition of mantle and core. Journal of Geophysical Research, 69, 4377-4388. doi: 10.1029/JZ069i020p04377.

Bizzarro, M., Baker, J. A., Haack, H., and Lundgaard, K. L., 2005. Rapid timescales for accretion and melting of differentiated planetesimals inferred from ${ }^{26} \mathrm{Al}-{ }^{26} \mathrm{Mg}$ chronometry. Astrophysical Journal, 632, L41-L44.

Bizzarro, M., Ulfbeck, D., Trinquier, A., Thrane, K., Connelly, J. N., and Meyer, B. S., 2007. Evidence for a late supernova injection of ${ }^{60} \mathrm{Fe}$ into the protoplanetary disk. Science, 316, 1178-1181. doi: 10.1126/science.1141040.

Bizzarro, M., Paton, C., Larsen, K., Schiller, M., Trinquier, A., and Ulfbeck, D., 2011. High-precision $\mathrm{Mg}$-isotope measurements of terrestrial and extraterrestrial material by HR-MC-ICPMS - implications for the relative and absolute mg-isotope composition of the bulk silicate earth. Journal of Analytical Atomic Spectrometry, 26, 565. doi: 10. 1039/c0ja00190b.

Borisov, A., Palme, H., and Spettel, B., 1994. Solubility of palladium in silicate melts: Implications for core formation in the earth. Geochimica et Cosmochimica Acta, 58, 705-716. doi: 10.1016/0016-7037(94)90500-2.

Bourdon, B., Quitté, G., and Halliday, A. N., 2006. Kinetic fractionation of nickel and iron between kamacite and taenite: Insights into cooling rates of iron meteorites. Meteoritics 
and Planetary Science Supplement, 41, 5263.

Brandon, A. D. and Walker, R. J., 2005. The debate over core-mantle interaction. Earth and Planetary Science Letters, 232, 211-225. doi: 16/j.epsl.2005.01.034.

Brandon, A. D., Walker, R. J., Morgan, J. W., Norman, M. D., and Prichard, H. M., 1998. Coupled ${ }^{186} \mathrm{Os}$ and ${ }^{187}$ Os evidence for Core-Mantle interaction. Science, 280, 1570 -1573. doi: 10.1126/science.280.5369.1570.

Brenan, J. M. and McDonough, W. F., 2005. Fractionation of highly siderophile elements (HSEs) by sulfide-silicate partitioning: A new spin. AGU Fall Meeting Abstracts, -1, 1502.

Brenan, J. M. and McDonough, W. F., 2009. Core formation and metal-silicate fractionation of osmium and iridium from gold. Nature Geoscience, 2, 798-801. doi: 10.1038/ngeo658.

Brenan, J., McDonough, W., and Dalpé, C., 2003. Experimental constraints on the partitioning of rhenium and some platinum-group elements between olivine and silicate melt. Earth and Planetary Science Letters, 212, 135-150. doi: 10.1016/S0012-821X(03) 00234-6.

Brett, R., 1971. The Earth's core: Speculations on its chemical equilibrium with the mantle. Geochimica et Cosmochimica Acta, 35, 203-221.

Brett, R., 1976. The current status of speculations on the composition of the core of the Earth. Reviews of Geophysics, 14, 375-383. doi: 10.1029/RG014i003p00375.

Brett, R., 1984. Chemical equilibration of the Earth's core and upper mantle. Geochimica et Cosmochimica Acta, 48, 1183-1188.

Briche, C. S. J., Taylor, P. D. P., and De Bièvre, P., 1997. Measurement of platinum isotope amount ratios by negative thermal ionization mass spectrometry using a thermionic quadrupole mass spectrometer. Anal. Chem., 69, 791-793. doi: 10.1021/ac9607368.

Burbine, T. H., McCoy, T. J., Meibom, A., Gladman, B., and Keil, K., 2002. Meteoritic parent bodies: Their number and identification. Asteroids III, 653, 667.

Burton, K. W., Gannoun, A., Birck, J.-L., Allègre, C. J., Schiano, P., Clocchiatti, R., and Alard, O., 2002. The compatibility of rhenium and osmium in natural olivine and their behaviour during mantle melting and basalt genesis. Earth and Planetary Science Letters, 198, 63-76. doi: 10.1016/S0012-821X(02)00518-6.

Cabri, L. J., 1992. The distribution of trace precious metals in minerals and mineral products. Mineralogical Magazine, 56, 289-308.

Cameron, A. G. W. and Truran, J. W., 1977. The supernova trigger for formation of the 
solar system. Icarus, 30, 447-461.

Cameron, A. G. W. and Ward, W. R., 1976. The Origin of the Moon. In Lunar and Planetary Institute Science Conference Abstracts, 7, 120.

Cameron, V., Vance, D., Archer, C., and House, C. H., 2009. A biomarker based on the stable isotopes of nickel. Proceedings of the National Academy of Sciences, 106, 10944-10948. doi: 10.1073/pnas.0900726106.

Canup, R. M., 2012. Forming a moon with an Earth-like composition via a giant impact. Science, 338, 1052-1055. doi: 10.1126/science.1226073.

Canup, R. M. and Asphaug, E., 2001. Origin of the moon in a giant impact near the end of the Earth's formation. Nature, 412, 708-712. doi: 10.1038/35089010.

Capobianco, C. J., Jones, J. H., and Drake, M. J., 1993. Metal-silicate thermochemistry at high temperature: Magma oceans and the "Excess siderophile element" problem of the Earth's upper mantles. Journal of Geophysical Research, 98, PP. 5433-5443. doi: 199310.1029/92JE02742.

Carlson, R. W., Boyet, M., and Horan, M., 2007. Chondrite barium, neodymium, and samarium isotopic heterogeneity and early Earth differentiation. Science, 316, 11751178. doi: 10.1126/science.1140189.

Catanzaro, E., Murphy, T., Shields, W., and Garner, E., 1968. Absolute isotopic abundance ratios of common, equal-atom, and radiogenic lead isotopic standards. Journal of Research of the National Bureau of Standards, 72A, 261-267.

Chabot, N. L. and Agee, C. B., 2003. Core formation in the Earth and Moon: new experimental constraints from V, Cr, and Mn. Geochimica et Cosmochimica Acta, 67, 2077-2091. doi: 10.1016/S0016-7037(02)01272-3.

Chakrabarti, R. and Jacobsen, S. B., 2009. A combined silicon and magnesium isotopic study of bulk meteorites and the Earth. In Lunar and Planetary Institute Science Conference Abstracts, 40, 2089.

Chakrabarti, R. and Jacobsen, S. B., 2010. Silicon isotopes in the inner solar system: Implications for core formation, solar nebular processes and partial melting. Geochimica et Cosmochimica Acta, 74, 6921-6933. doi: 10.1016/j.gca.2010.08.034.

Chambers, J., 2007. Planet formation. In Treatise on Geochemistry, Holland, H. D. and Turekian, K. K. (Eds.) 1-17. Pergamon, Oxford.

Chen, J. H. and Wasserburg, G. J., 1990. The isotopic composition of Ag in meteorites and the presence of ${ }^{107} \mathrm{Pd}$ in protoplanets. Geochimica et Cosmochimica Acta, 54, $1729-1743$. 
Chen, J., Papanastassiou, D., and Wasserburg, G., 2009. A search for nickel isotopic anomalies in iron meteorites and chondrites. Geochimica et Cosmochimica Acta, 73, 1461-1471. doi: 10.1016/j.gca.2008.11.040.

Chou, C., 1978. Fractionation of siderophile elements in the Earth's upper mantles. In Proceedings of the 9th Lunar and Planetary Science Conference, 1, 219-230.

Clarke, F. W., 1920. The Data of Geochemistry. U.S. Government Printing Office.

Clayton, R. N., Mayeda, T. K., and Nagahara, H., 1992. Oxygen isotope relationships among primitive achondrites. In Lunar and Planetary Institute Science Conference Abstracts, 23, 231.

Clayton, R., 2003. Oxygen isotopes in meteorites. In Treatise on Geochemistry, Heinrich D. Holland and Karl K. Turekian (Eds.) 129-142. Pergamon, Oxford.

Clayton, R. N. and Mayeda, T. K., 1988. Formation of ureilites by nebular processes. Geochimica et Cosmochimica Acta, 52, 1313-1318. doi: 10.1016/0016-7037(88)90286-4.

Clayton, R. N. and Mayeda, T. K., 1996. Oxygen isotope studies of achondrites. Geochimica et Cosmochimica Acta, 60, 1999-2017. doi: 10.1016/0016-7037(96)00074-9.

Clayton, R. N., Mayeda, T. K., and Rubin, A. E., 1984. Oxygen isotopic compositions of enstatite chondrites and aubrites. Journal of Geophysical Research: Solid Earth, 89, C245-C249. doi: 10.1029/JB089iS01p0C245.

Colodner, D. The Marine Geochemistry of Rhenium, Iridium and Platinum. PhD, Woods Hole Oceanographic Institute, MA, 1991.

Compston, W. and Oversby, V. M., 1969. Lead isotopic analysis using a double spike. Journal of Geophysical Research, 74, 4338-4348. doi: 196910.1029/JB074i017p04338.

Connelly, J. N., Bizzarro, M., Krot, A. N., Nordlund, Å., Wielandt, D., and Ivanova, M. A., 2012. The absolute chronology and thermal processing of solids in the solar protoplanetary disk. Science, 338, 651-655. doi: 10.1126/science.1226919.

Connelly, J., Bizzarro, M., Thrane, K., and Baker, J., 2008. The Pb-Pb age of angrite SAH99555 revisited. Geochimica et Cosmochimica Acta, 72, 4813-4824. doi: 10.1016/ j.gca.2008.06.007.

Cook, D. L., Walker, R. J., Horan, M. F., Wasson, J. T., and Morgan, J. W., 2004. Pt-ReOs systematics of group IIAB and IIIAB iron meteorites. Geochimica et Cosmochimica Acta, 68, 1413-1431.

Cook, D. L., Wadhwa, M., Clayton, R. N., Janney, P. E., Dauphas, N., and Davis, A. M., 2006a. Mass-dependent fractionation of nickel isotopes in IIIAB iron meteorites. Meteoritics and Planetary Science Supplement, 41, 5167. 
Cook, D. L., Wadhwa, M., Janney, P. E., Dauphas, N., Clayton, R. N., and Davis, A. M., 2006b. High precision measurements of non-mass-dependent effects in nickel isotopes in meteoritic metal via multicollector ICPMS. Analytical Chemistry, 78, 8477-8484. doi: $10.1021 /$ ac061285m.

Cook, D. L., Wadhwa, M., Clayton, R. N., Dauphas, N., Janney, P. E., and Davis, A. M., 2007. Mass-dependent fractionation of nickel isotopes in meteoritic metal. Meteoritics $\&$ Planetary Science, 42, 2067-2077. doi: 10.1111/j.1945-5100.2007.tb01008.x.

Cook, D. L., Clayton, R. N., Wadhwa, M., Janney, P. E., and Davis, A. M., 2008. Nickel isotopic anomalies in troilite from iron meteorites. Geophysical Research Letters, 35. doi: 10.1029/2007GL032431.

Coplen, T. B., 1997. Atomic weights of the elements 1995. Journal of Physical and Chemical Reference Data, 26, 1239-1253. doi: 10.1063/1.556001.

Cotton, F. A. and Wilkinson, G., 1966. Advanced inorganic chemistry: a comprehensive text. Interscience Publishers.

Cottrell, E. and Walker, D., 2006. Constraints on core formation from Pt partitioning in mafic silicate liquids at high temperatures. Geochimica et Cosmochimica Acta, 70, 1565-1580. doi: 16/j.gca.2005.11.021.

Cottrell, E., Walter, M. J., and Walker, D., 2009. Metal-silicate partitioning of tungsten at high pressure and temperature: Implications for equilibrium core formation in Earth. Earth and Planetary Science Letters, 281, 275-287. doi: 10.1016/j.epsl.2009.02.024.

Creech, J., Baker, J., Handler, M., and Bizzarro, M., 2014. Platinum stable isotope analysis of geological standard reference materials by double-spike MC-ICPMS. Chemical Geology, 363, 293-300. doi: 10.1016/j.chemgeo.2013.11.009.

Creech, J. and Paul, B., 2014. IsoSpike: Improved double-spike deconvolution software. Geostandards and Geoanalytical Research, In press. doi: 10.1111/j.1751-908X.2014. 00276.x.

Creech, J., Baker, J., Handler, M., Schiller, M., and Bizzarro, M., 2013. Platinum stable isotope ratio measurements by double-spike multiple collector ICPMS. Journal of Analytical Atomic Spectrometry, 28, 853-865. doi: 10.1039/C3JA50022E.

Ćuk, M. and Stewart, S. T., 2012. Making the moon from a fast-spinning Earth: A Giant Impact followed by resonant despinning. Science, 338, 1047-1052. doi: 10.1126/science. 1225542

Dale, C. W., Burton, K. W., Greenwood, R. C., Gannoun, A., Wade, J., Wood, B. J., and Pearson, D. G., 2012. Late accretion on the earliest planetesimals revealed by the highly siderophile elements. Science, 336, 72-75. doi: 10.1126/science.1214967. 
Dauphas, N. and Pourmand, A., 2011. Hf-W-Th evidence for rapid growth of Mars and its status as a planetary embryo. Nature, 473, 489-492. doi: 10.1038/nature10077.

Dauphas, N., 2007. Diffusion-driven kinetic isotope effect of Fe and Ni during formation of the Widmanstätten pattern. Meteoritics \& Planetary Science, 42, 1597-1613.

Dauphas, N. and Marty, B., 2002. Inference on the nature and the mass of Earth's late veneer from noble metals and gases. Journal of Geophysical Research, 107, 7. doi: 200210.1029/2001JE001617.

Dauphas, N. and Rouxel, O., 2006. Mass spectrometry and natural variations of iron isotopes. Mass Spectrometry Reviews, 25, 515-550. doi: 10.1002/mas.20078.

Dauphas, N., Davis, A. M., Marty, B., and Reisberg, L., 2004a. The cosmic molybdenumruthenium isotope correlation. Earth and Planetary Science Letters, 226, 465-475. doi: 10.1016/j.epsl.2004.07.026.

Dauphas, N., Janney, P. E., Mendybaev, R. A., Wadhwa, M., Richter, F. M., Davis, A. M., van Zuilen, M., Hines, R., and Foley, C. N., 2004b. Chromatographic separation and multicollection-ICPMS analysis of iron. investigating mass-dependent and-independent isotope effects. Analytical Chemistry, 76, 5855-5863.

Dauphas, N., van Zuilen, M., Wadhwa, M., Davis, A. M., Marty, B., and Janney, P. E., 2004c. Clues from Fe isotope variations on the origin of early Archean BIFs from Greenland. Science, 306, 2077-2080.

Day, J. M. D., Walker, R. J., Qin, L., and Rumble III, D., 2012. Late accretion as a natural consequence of planetary growth. Nature Geoscience, 5, 614-617. doi: 10.1038/ ngeo1527.

Day, J. M., 2013. Hotspot volcanism and highly siderophile elements. Chemical Geology, 341, 50-74. doi: 10.1016/j.chemgeo.2012.12.010.

De Biévre, P., Gallet, M., Holden, N. E., and Barnes, I. L., 1984. Isotopic abundances and atomic weights of the elements. Journal of Physical and Chemical Reference Data, 13, 809-891. doi: doi:10.1063/1.555720.

Dideriksen, K., Baker, J., and Stipp, S., 2006. Iron isotopes in natural carbonate minerals determined by MC-ICP-MS with a ${ }^{58} \mathrm{Fe}-{ }^{54} \mathrm{Fe}$ double spike. Geochimica et Cosmochimica Acta, 70, 118-132. doi: 10.1016/j.gca.2005.08.019.

Dodson, M. H., 1963. A theoretical study of the use of internal standards for precise isotopic analysis by the surface ionization technique: Part I - general first-order algebraic solutions. Journal of Scientific Instruments, 40, 289-295.

Dole, M. and Slobod, R. L., 1940. Isotopic composition of oxygen in carbonate rocks and 
iron oxide ores. Journal of the American Chemical Society, 62, 471-479.

D'Orazio, M. and Folco, L., 2003. Chemical analysis of iron meteorites by inductively coupled plasma-mass spectrometry. Geostandards and Geoanalytical Research, 27, 215225. doi: 10.1111/j.1751-908X.2003.tb00723.x.

Drake, M. J., 2000. Accretion and primary differentiation of the Earth: a personal journey. Geochimica et Cosmochimica Acta, 64, 2363-2369. doi: 10.1016/S0016-7037(00)00372-0.

Drake, M. J. and Righter, K., 2002. Determining the composition of the Earth. Nature, 416, 39-44. doi: 10.1038/416039a.

Drake, M. J., Newsom, H. E., and Capobianco, C. J., 1989. V, Cr, and Mn in the Earth, Moon, EPB, and SPB and the origin of the Moon: Experimental studies. Geochimica et Cosmochimica Acta, 53, 2101-2111.

Ek, K. H., Morrison, G. M., and Rauch, S., 2004. Environmental routes for platinum group elements to biological materials - a review. Science of The Total Environment, 334-335, 21-38. doi: 10.1016/j.scitotenv.2004.04.027.

Ellis, A. S., Johnson, T. M., and Bullen, T. D., 2002. Chromium isotopes and the fate of hexavalent chromium in the environment. Science, 295, 2060-2062. doi: 10.1126/ science.1068368.

Ellis, A. S., Johnson, T. M., and Bullen, T. D., 2004. Using chromium stable isotope ratios to quantify $\mathrm{Cr}(\mathrm{VI})$ reduction: Lack of sorption effects. Environmental Science $\&$ Technology, 38, 3604-3607. doi: 10.1021/es0352294.

Ely, J. C., Neal, C. R., Kulpa, C. F., Schneegurt, M. A., Seidler, J. A., and Jain, J. C., 2001. Implications of Platinum-Group element accumulation along U.S. roads from Catalytic-Converter attrition. Environmental Science \& Technology, 35, 3816-3822. doi: $10.1021 /$ es001989s.

Emteborg, H., Tian, X., Ostermann, M., Berglund, M., and Adams, F. C., 2000. Isotope ratio and isotope dilution measurements using axial inductively coupled plasma time of flight mass spectrometry. Journal of Analytical Atomic Spectrometry, 15, 239-246. doi: 10.1039/A909575F.

Endress, M. and Bischoff, A., 1993. Mineralogy, degree of brecciation, and aqueous alteration of CI chondrites Orgueil, Ivuna, and Alais. Meteoritics, 28, 345-346.

Endress, M. and Bischoff, A., 1996. Carbonates in CI chondrites: Clues to parent body evolution. Geochimica et Cosmochimica Acta, 60, 489-507. doi: 10.1016/0016-7037(95) 00399-1.

Endress, M., Zinner, E., and Bischoff, A., 1996. Early aqueous activity on primitive 
meteorite parent bodies. Nature, 379, 701-703. doi: 10.1038/379701a0.

Ertel, W., O'Neill, H., Sylvester, P., and Dingwell, D., 1999. Solubilities of Pt and Rh in a haplobasaltic silicate melt at $1300^{\circ} \mathrm{C}$. Geochimica et Cosmochimica Acta, 63, 2439-2449. doi: 10.1016/S0016-7037(99)00136-2.

Ertel, W., Walter, M. J., Drake, M. J., and Sylvester, P. J., 2006. Experimental study of platinum solubility in silicate melt to $14 \mathrm{GPa}$ and $2273 \mathrm{~K}$ : Implications for accretion and core formation in Earth. Geochimica et Cosmochimica Acta, 70, 2591-2602. doi: 10.1016/j.gca.2006.02.015.

Eucken, A., 1944. Physikalisch-chemische betrachtungen über die frühste entwicklungsgeschichte der erde. Nachrichten Akademischen Wissenschaften Göttingen 1-25.

Eugster, O., 2003. Cosmic-ray exposure ages of meteorites and lunar rocks and their significance. Chemie der Erde - Geochemistry, 63, 3-30. doi: 10.1078/0009-2819-00021.

Eugster, O., Tera, F., and Wasserburg, G. J., 1969. Isotopic analyses of barium in meteorites and in terrestrial samples. Journal of Geophysical Research, 74, 3897-3908. doi: 196910.1029/JB074i015p03897.

Eugster, O., Herzog, G. F., Marti, K., and Caffee, M. W., 2006. Irradiation records, cosmic-ray exposure ages, and transfer times of meteorites. Meteorites and the early solar system II, 1, 829-851.

Evans, N. and Chai, C., 1997. The distribution and geochemistry of platinum-group elements as event markers in the phanerozoic. Palaeogeography, Palaeoclimatology, Palaeoecology, 132, 373-390. doi: 10.1016/S0031-0182(97)00059-X.

Faure, G., 1998. Principles and applications of geochemistry: a comprehensive textbook for geology students. Prentice Hall New Jersey.

Faye, G. H. and Sutarno, R., 1976. Certified compositional reference materials for the Earth sciences. The Canadian Mineralogist, 14, 164-171.

Findlay, D. C., 1969. Origin of the Tulameen ultramafic-gabbro complex, southern British Columbia. Canadian Journal of Earth Sciences, 6, 399-425. doi: 10.1139/e69-040.

Fischer-Gödde, M., Becker, H., and Wombacher, F., 2011. Rhodium, gold and other highly siderophile elements in orogenic peridotites and peridotite xenoliths. Chemical Geology, 280, 365-383. doi: 10.1016/j.chemgeo.2010.11.024.

Fitoussi, C. and Bourdon, B., 2012. Silicon isotope evidence against an enstatite chondrite Earth. Science, 335, 1477-1480. doi: 10.1126/science.1219509.

Fitoussi, C., Bourdon, B., Kleine, T., Oberli, F., and Reynolds, B. C., 2009. Si isotope systematics of meteorites and terrestrial peridotites: implications for $\mathrm{Mg} / \mathrm{Si}$ fractiona- 
tion in the solar nebula and for Si in the Earth's core. Earth and Planetary Science Letters, 287, 77-85. doi: 10.1016/j.epsl.2009.07.038.

Fleet, M., Crocket, J., Liu, M., and Stone, W., 1999a. Laboratory partitioning of platinumgroup elements (PGE) and gold with application to magmatic sulfide-PGE deposits. Lithos, 47, 127-142. doi: 10.1016/S0024-4937(99)00011-0.

Fleet, M., Liu, M., and Crocket, J., 1999b. Partitioning of trace amounts of highly siderophile elements in the $\mathrm{Fe}-\mathrm{Ni}-\mathrm{S}$ system and their fractionation in nature. Geochimica et Cosmochimica Acta, 63, 2611-2622. doi: 10.1016/S0016-7037(99)00112-X.

Fortenfant, S. S., Günther, D., Dingwell, D. B., and Rubie, D. C., 2003. Temperature dependence of Pt and Rh solubilities in a haplobasaltic melts. Geochimica et Cosmochimica Acta, 67, 123-131. doi: 10.1016/S0016-7037(02)01047-5.

Frei, R., Gaucher, C., Poulton, S. W., and Canfield, D. E., 2009. Fluctuations in Precambrian atmospheric oxygenation recorded by chromium isotopes. Nature, 461, 250-253. doi: 10.1038/nature08266.

Galer, S. J. G., 1999. Optimal double and triple spiking for high precision lead isotopic measurement. Chemical Geology, 157, 255-274. doi: 10.1016/S0009-2541(98)00203-4.

Gall, L., Williams, H., Siebert, C., and Halliday, A., 2012. Determination of massdependent variations in nickel isotope compositions using double spiking and MCICPMS. Journal of Analytical Atomic Spectrometry, 27, 137. doi: 10.1039/c1ja10209e.

Gammons, C. H., 1996. Experimental investigations of the hydrothermal geochemistry of platinum and palladium: V. equilibria between platinum metal, $\mathrm{Pt}(\mathrm{II})$, and $\mathrm{Pt}(\mathrm{IV})$ chloride complexes at 25 to $300^{\circ} \mathrm{C}$. Geochimica et Cosmochimica Acta, 60, 1683-1694. doi: 10.1016/0016-7037(96)00048-8.

Georg, R. B., Halliday, A. N., Schauble, E. A., and Reynolds, B. C., 2007. Silicon in the Earth's core. Nature, 447, 1102-1106. doi: 10.1038/nature05927.

Gessmann, C. K., Wood, B. J., Rubie, D. C., and Kilburn, M. R., 2001. Solubility of silicon in liquid metal at high pressure: implications for the composition of the Earth's core. Earth and Planetary Science Letters, 184, 367-376.

Godel, B., Barnes, S.-J., and Maier, W. D., 2007. Platinum-group elements in sulphide minerals, platinum-group minerals, and whole-rocks of the Merensky Reef (Bushveld Complex, South Africa): Implications for the formation of the reef. Journal of Petrology, 48, 1569-1604. doi: 10.1093/petrology/egm030.

Goldberg, E. D., 1987. Comparative chemistry of the platinum and other heavy metals in the marine environment. Pure and applied chemistry, 59, 565-571. 
Goldberg, E. D. and Koide, M., 1990. Understanding the marine chemistries of the platinum group metals. Marine Chemistry, 30, 249-257. doi: 10.1016/0304-4203(90) 90074-M.

Goldberg, E. D., Hodge, V., Kay, P., Stallard, M., and Koide, M., 1986. Some comparative marine chemistries of platinum and iridium. Applied Geochemistry, 1, 227-232. doi: 10.1016/0883-2927(86)90006-5.

Goldstein, J., Scott, E., and Chabot, N., 2009. Iron meteorites: Crystallization, thermal history, parent bodies, and origin. Chemie der Erde - Geochemistry, 69, 293-325. doi: 10.1016/j.chemer.2009.01.002.

Goldstein, J. I. and Ogilvie, R. E., 1965. The growth of the Widmanstätten pattern in metallic meteorites. Geochimica et Cosmochimica Acta, 29, 893-920.

Gomes, R., Levison, H. F., Tsiganis, K., and Morbidelli, A., 2005. Origin of the cataclysmic late heavy bombardment period of the terrestrial planets. Nature, 435, 466-469. doi: 10.1038 /nature03676.

Goodrich, C. A., Jones, J. H., and Spitz, A. H., 1987. Siderophile element tests of ureilite petrogenesis models. Meteoritics, 22, 392 .

Goodrich, C. A., 1992. Ureilites: A critical review. Meteoritics, 27, 327-352. doi: 10. 1111/j.1945-5100.1992.tb00215.x.

Goodrich, C. A., Scott, E. R., and Fioretti, A. M., 2004. Ureilitic breccias: clues to the petrologic structure and impact disruption of the ureilite parent asteroid. Chemie der Erde - Geochemistry, 64, 283-327. doi: 10.1016/j.chemer.2004.08.001.

Gopalan, K., Macdougall, D., and Macisaac, C., 2006. Evaluation of a ${ }^{42} \mathrm{Ca}-{ }^{43} \mathrm{Ca}$ doublespike for high precision Ca isotope analysis. International Journal of Mass Spectrometry, 248, 9-16. doi: 10.1016/j.ijms.2005.09.009.

Graf, T. and Marti, K., 1992. Cosmic-ray exposure history of enstatite meteorites. Meteoritics, $27,227$.

Graf, T. and Marti, K., 1994. Collisional records in LL-chondrites. Meteoritics, 29, 643-648. doi: 10.1111/j.1945-5100.1994.tb00777.x.

Greenough, J. D. and Owen, J., 1992. Platinum-group element geochemistry of continental tholeiites: Analysis of the long range dyke swarm, newfoundland, canada. Chemical Geology, 98, 203-219. doi: 10.1016/0009-2541(92)90185-8.

Grevesse, N. and Sauval, A. J., 1998. Standard solar composition. Space Science Reviews, 85, 161-174. doi: 10.1023/A:1005161325181.

Gros, M., Lorand, J., and Luguet, A., 2002. Analysis of platinum group elements and gold 
in geological materials using NiS fire assay and Te coprecipitation; the NiS dissolution step revisited. Chemical Geology, 185, 179-190. doi: 16/S0009-2541(01)00405-3.

Guan, Y. and Crozaz, G., 2001. Microdistributions and petrogenetic implications of rare earth elements in polymict ureilites. Meteoritics $\mathcal{E} 3$ Planetary Science, 36, 1039-1056.

Gueguen, B., Rouxel, O., Ponzevera, E., Bekker, A., and Fouquet, Y., 2013. Nickel isotope variations in terrestrial silicate rocks and geological reference materials measured by MCICP-MS. Geostandards and Geoanalytical Research 1-21. doi: 10.1111/j.1751-908X. 2013.00209.x.

Guo, Z., Sadler, P. J., and Sykes, A., 1999. Medicinal inorganic chemistry. In Advances in Inorganic Chemistry 49 183-306. Academic Press. doi: 10.1016/S0898-8838(08)60271-8.

Haack, H. and McCoy, T., 2007. Iron and stony-iron meteorites. In Treatise on Geochemistry, Holland, H. D. and Turekian, K. K. (Eds.) 1-22. Pergamon, Oxford.

Haack, H. and Scott, E. R., 1993. Chemical fractionations in group IIIAB iron meteorites: Origin by dendritic crystallization of an asteroidal core. Geochimica et Cosmochimica Acta, 57, 3457-3472. doi: 10.1016/0016-7037(93)90550-G.

Hagen, J. C. Some aspects of the geochemistry of platinum, palladium, and gold in igneous rocks with special reference to the Bushveld Complex, Transvaal. Ph.D., Massachusetts Institute of Technology, 1954.

Halbach, P., Kriete, C., Prause, B., and Puteanus, D., 1989. Mechanisms to explain the platinum concentration in ferromanganese seamount crusts. Chemical Geology, 76, 95-106. doi: 10.1016/0009-2541(89)90130-7.

Halliday, A. N., Lee, D.-C., and Jacobsen, S. B., 2000. Tungsten isotopes, the timing of metal-silicate fractionation, and the origin of the Earth and Moon. In Origin of the Earth and Moon, Canup, R. and Righter, K. (Eds.) 45-62. University of Arizona Press, Tucson.

Halliday, A. N., Wänke, H., Birck, J.-L., and Clayton, R. N., 2001. The accretion, composition and early differentiation of Mars. Space Science Reviews, 96, 197-230. doi: 10.1023/A:1011997206080.

Halliday, A. N., 2008. A young moon-forming giant impact at 70-110 million years accompanied by late-stage mixing, core formation and degassing of the Earth. Philosophical Transactions of the Royal Society A: Mathematical, Physical and Engineering Sciences, 366, 4163 -4181. doi: 10.1098/rsta.2008.0209.

Halliday, A. N. and Wood, B. J., 2009. How did Earth accrete? Science, 325, 44-45. doi: 10.1126/science.1172587. 
Halliday, A. N., Lee, D.-C., Christensen, J. N., Rehkämper, M., Yi, W., Luo, X., Hall, C. M., Ballentine, C. J., Pettke, T., and Stirling, C., 1998. Applications of multiple collector-ICPMS to cosmochemistry, geochemistry, and paleoceanography. Geochimica et Cosmochimica Acta, 62, 919-940. doi: 10.1016/S0016-7037(98)00057-X.

Halliday, A., 2003. The origin and earliest history of the earth. In Treatise on Geochemistry, Heinrich D. Holland and Karl K. Turekian (Eds.) 509-557. Pergamon, Oxford.

Hamelin, B., Manhes, G., Albarede, F., and Allègre, C. J., 1985. Precise lead isotope measurements by the double spike technique: A reconsideration. Geochimica et Cosmochimica Acta, 49, 173-182. doi: 10.1016/0016-7037(85)90202-9.

Handler, M. R. and Bennett, V. C., 1999. Behaviour of platinum-group elements in the subcontinental mantle of eastern Australia during variable metasomatism and melt depletion. Geochimica et Cosmochimica Acta, 63, 3597-3618.

Handler, M., Bennett, V., and Carlson, R., 2005. Nd, Sr and Os isotope systematics in young, fertile spinel peridotite xenoliths from northern Queensland, Australia: A unique view of depleted MORB mantle? Geochimica et Cosmochimica Acta, 69, 5747-5763. doi: $10.1016 /$ j.gca.2005.08.003.

Hart, T. R. and MacDonald, C. A., 2007. Proterozoic and Archean geology of the Nipigon Embayment: implications for emplacement of the Mesoproterozoic Nipigon diabase sills and mafic to ultramafic intrusions. Canadian Journal of Earth Sciences, 44, 1021-1040. doi: 10.1139/E07-026.

Hartmann, W. K., Ryder, G., Dones, L., and Grinspoon, D., 2000. The time-dependent intense bombardment of the primordial Earth/Moon system. Origin of the Earth and Moon, -1, 493-512.

Hartmann, W. K., 1975. Lunar "cataclysm": A misconception? Icarus, 24, 181-187.

Hartmann, W. K., 2003. Megaregolith evolution and cratering cataclysm models-Lunar cataclysm as a misconception (28 years later). Meteoritics $\&$ Planetary Science, 38, 579-593. doi: 10.1111/j.1945-5100.2003.tb00028.x.

Hartmann, W. K. and Davis, D. R., 1975. Satellite-sized planetesimals and lunar origin. Icarus, 24, 504-515. doi: 10.1016/0019-1035(75)90070-6.

Hattori, K., Menagh, D. P., and Cole, T. J. S., 1998. Low blank isotope ratio measurements of rhenium, osmium, and platinum using tantalum filaments with negative thermal ionization mass spectrometry. Analytical Chemistry, 70, 4100-4103. doi: 10.1021/ ac971037d

Heaman, L., Easton, R., Hart, T., Hollings, P., MacDonald, C., and Smyk, M., 2007. Further refinement to the timing of Mesoproterozoic magmatism, Lake Nipigon region, 
Ontario. Canadian Journal of Earth Sciences, 44, 1055-1086. doi: 10.1139/E06-117.

Heck, R. M. and Farrauto, R. J., 1995. Catalytic air pollution control: commercial technology. Van Nostrand Reinhold, New York, USA. ISBN 9780442017828.

Herzog, G., 2007. 1.13 - cosmic-ray exposure ages of meteorites. In Treatise on Geochemistry, Editors-in-Chief: Heinrich D. Holland and Karl K. Turekian (Eds.) 1-36. Pergamon, Oxford.

Hillgren, V. J., Drake, M. J., and Rubie, D. C., 1996. High pressure and high temperature metal-silicate partitioning of siderophile elements: The importance of silicate liquid composition. Geochimica et Cosmochimica Acta, 60, 2257-2263. doi: 10.1016/0016-7037(96)00079-8.

Ho, Y., Au-Yeung, S. C. F., and To, K. K. W., 2003. Platinum-based anticancer agents: Innovative design strategies and biological perspectives. Medicinal Research Reviews, 23, 633-655. doi: 10.1002/med.10038.

Hodge, V. F., Stallard, M., Koide, M., and Goldberg, E. D., 1985. Platinum and the platinum anomaly in the marine environment. Earth and Planetary Science Letters, 72, 158-162. doi: 10.1016/0012-821X(85)90002-0.

Holzheid, A., Sylvester, P., O’Neill, H. S., Rubie, D. C., and Palme, H., 2000. Evidence for a late chondritic veneer in the Earth's mantle from high-pressure partitioning of palladium and platinum. Nature, 406, 396-399. doi: 10.1038/35019050.

Hopfe, W. D. and Goldstein, J. I., 2001. The metallographic cooling rate method revised: Application to iron meteorites and mesosiderites. Meteoritics $\&$ Planetary Science, 36 , $135-154$.

Horan, M., Walker, R. J., Morgan, J. W., Grossman, J., and Rubin, A., 2003. Highly siderophile elements in chondrites. Chemical Geology, 196, 27-42. doi: 10.1016/ S0009-2541(02)00405-9.

Horn, I., von Blanckenburg, F., Schoenberg, R., Steinhoefel, G., and Markl, G., 2006. In situ iron isotope ratio determination using UV-femtosecond laser ablation with application to hydrothermal ore formation processes. Geochimica et Cosmochimica Acta, 70, 3677-3688. doi: 10.1016/j.gca.2006.05.002.

Houlé, M. G., Préfontaine, S., Fowler, A. D., and Gibson, H. L., 2009. Endogenous growth in channelized komatiite lava flows: evidence from spinifex-textured sills at Pyke Hill and Serpentine Mountain, Western Abitibi Greenstone Belt, Northeastern Ontario, Canada. Bulletin of Volcanology, 71, 881-901. doi: 10.1007/s00445-009-0273-y.

Jaeger, W. L. and Drake, M. J., 2000. Metal-silicate partitioning of Co, Ga, and W: dependence on silicate melt compositions. Geochimica et Cosmochimica Acta, 64, 3887- 
3895. doi: 10.1016/S0016-7037(00)00489-0.

Jagoutz, E., Palme, H., Baddenhausen, H., Blum, K., Cendales, M., Dreibus, G., Spettel, B., Waenke, H., and Lorenz, V., 1979. The abundances of major, minor and trace elements in the earth's mantle as derived from primitive ultramafic nodules. In Lunar and Planetary Science Conference Proceedings, Hinners, N. W. (Ed.), 10, 2031-2050.

Jaireth, S., 1992. The calculated solubility of platinum and gold in oxygen-saturated fluids and the genesis of platinum-palladium and gold mineralization in the unconformityrelated uranium deposits. Mineralium Deposita, 27, 42-54. doi: 10.1007/BF00196079.

Janssens, M.-J., Hertogen, J., Wolf, R., Ebihara, M., and Anders, E., 1987. Ureilites: Trace element clues to their origin. Geochimica et Cosmochimica Acta, 51, 2275-2283. doi: 10.1016/0016-7037(87)90280-8.

Javoy, M., 1995. The integral enstatite chondrite model of the Earth. Geophysical Research Letters, 22, 2219-2222.

Javoy, M., Kaminski, E., Guyot, F., Andrault, D., Sanloup, C., Moreira, M., Labrosse, S., Jambon, A., Agrinier, P., Davaille, A., and Jaupart, C., 2010. The chemical composition of the Earth: Enstatite chondrite models. Earth and Planetary Science Letters, 293, 259-268. doi: 10.1016/j.epsl.2010.02.033.

Johnson, C. M. and Beard, B. L., 1999. Correction of instrumentally produced mass fractionation during isotopic analysis of Fe by thermal ionization mass spectrometry. International Journal of Mass Spectrometry, 193, 87-99. doi: 10.1016/S1387-3806(99) 00158-X.

Johnson, C. M., Beard, B. L., and Albarède, F., 2004. Overview and general concepts. Reviews in Mineralogy and Geochemistry, 55, 1-24. doi: 10.2138/gsrmg.55.1.1.

Johnson, T. M. and Bullen, T. D., 2004. Mass-dependent fractionation of selenium and chromium isotopes in low-temperature environments. Reviews in Mineralogy and Geochemistry, 55, 289-317. doi: 10.2138/gsrmg.55.1.289.

Jones, J. H. and Drake, M. J., 1986. Geochemical constraints on core formation in the Earth. Nature, 322, 221-228. doi: 10.1038/322221a0.

Kašpar, J., Fornasiero, P., and Hickey, N., 2003. Automotive catalytic converters: current status and some perspectives. Catalysis Today, 77, 419-449. doi: 10.1016/ S0920-5861(02)00384-X.

Kehm, K., Hauri, E., Alexander, C., and Carlson, R., 2003. High precision iron isotope measurements of meteoritic material by cold plasma ICP-MS. Geochimica et Cosmochimica Acta, 67, 2879-2891. doi: 10.1016/S0016-7037(03)00080-2. 
Keil, K., 1968. Mineralogical and chemical relationships among enstatite chondrites. Journal of Geophysical Research, 73, 6945-6976.

Kilburn, M. and Wood, B., 1997. Metal-silicate partitioning and the incompatibility of S and Si during core formation. Earth and Planetary Science Letters, 152, 139-148. doi: 10.1016/S0012-821X(97)00125-8.

Kimura, K., Lewis, R. S., and Anders, E., 1974. Distribution of gold and rhenium between nickel-iron and silicate melts: implications for the abundance of siderophile elements on the Earth and Moon. Geochimica et Cosmochimica Acta, 38, 683-701. doi: 10.1016/ 0016-7037(74)90144-6.

Kleine, T., Munker, C., Mezger, K., and Palme, H., 2002. Rapid accretion and early core formation on asteroids and the terrestrial planets from Hf-W chronometry. Nature, 418, 952-955. doi: 10.1038/nature00982.

Kleine, T., Mezger, K., Münker, C., Palme, H., and Bischoff, A., 2004a. ${ }^{182} \mathrm{Hf}-{ }^{182} \mathrm{~W}$ isotope systematics of chondrites, eucrites, and martian meteorites: Chronology of core formation and early mantle differentiation in Vesta and Mars. Geochimica et Cosmochimica Acta, 68, 2935-2946. doi: 10.1016/j.gca.2004.01.009.

Kleine, T., Mezger, K., Palme, H., and Münker, C., 2004b. The W isotope evolution of the bulk silicate earth: constraints on the timing and mechanisms of core formation and accretion. Earth and Planetary Science Letters, 228, 109-123. doi: 10.1016/j.epsl.2004. 09.023

Kleine, T., 2011. Geoscience: Earth's patchy late veneer. Nature, 477, 168-169. doi: 10.1038/477168a.

Kokubo, E. and Ida, S., 2002. Formation of protoplanet systems and diversity of planetary systems. The Astrophysical Journal, 581, 666. doi: 10.1086/344105.

Kring, D. A. and Cohen, B. A., 2002. Cataclysmic bombardment throughout the inner solar system 3.9-4.0 Ga. Journal of Geophysical Research: Planets, 107, 4-1-4-6. doi: 10.1029/2001JE001529.

Krot, A., Keil, K., Scott, E., Goodrich, C., and Weisberg, M., 2007. Classification of meteorites. In Treatise on Geochemistry, Holland, H. D. and Turekian, K. K. (Eds.) 1-52. Pergamon, Oxford.

Kruijer, T. S., Fischer-Gödde, M., Kleine, T., Sprung, P., Leya, I., and Wieler, R., 2013. Neutron capture on Pt isotopes in iron meteorites and the Hf-W chronology of core formation in planetesimals. Earth and Planetary Science Letters, 361, 162-172. doi: 10.1016/j.epsl.2012.10.014.

Labidi, J., Cartigny, P., and Moreira, M., 2013. Non-chondritic sulphur isotope com- 
position of the terrestrial mantle. Nature, advance online publication. doi: 10.1038/ nature12490.

Lazar, C., Young, E. D., and Manning, C. E., 2012. Experimental determination of equilibrium nickel isotope fractionation between metal and silicate from $500^{\circ} \mathrm{C}$ to $950{ }^{\circ} \mathrm{C}$. Geochimica et Cosmochimica Acta, 86, 276-295. doi: 10.1016/j.gca.2012.02.024.

Leya, I., Schönbächler, M., Wiechert, U., Krähenbühl, U., and Halliday, A. N., 2008. Titanium isotopes and the radial heterogeneity of the solar system. Earth and Planetary Science Letters, 266, 233-244. doi: 10.1016/j.epsl.2007.10.017.

Li, J. and Agee, C., 2001. The effect of pressure, temperature, oxygen fugacity and composition on partitioning of nickel and cobalt between liquid Fe-Ni-S alloy and liquid silicate: implications for the Earth's core formation. Geochimica et Cosmochimica Acta, 65, 1821-1832. doi: 10.1016/S0016-7037(00)00613-X.

Li, J. and Fei, Y., 2007. Experimental constraints on core compositions. In Treatise on Geochemistry, Heinrich D. Holland and Karl K. Turekian (Eds.) 1-31. Pergamon, Oxford.

Li, J. and Agee, C. B., 1996. Geochemistry of mantle-core differentiation at high pressure. Nature, 381, 686-689. doi: 10.1038/381686a0.

Li, Y.-H., 1991. Distribution patterns of the elements in the ocean: A synthesis. Geochimica et Cosmochimica Acta, 55, 3223-3240. doi: 10.1016/0016-7037(91)90485-N.

Lorand, J.-P. and Alard, O., 2001. Platinum-group element abundances in the upper mantle: new constraints from in situ and whole-rock analyses of massif central xenoliths (France). Geochimica et Cosmochimica Acta, 65, 2789-2806. doi: 10.1016/S0016-7037(01)00627-5.

Lorand, J., Luguet, A., and Alard, O., 2008. Platinum-Group elements: A new set of key tracers for the Earth's interior. Elements, 4, 247-252. doi: 10.2113/GSELEMENTS.4. 4.247 .

Lorand, J.-P., Alard, O., and Luguet, A., 2010. Platinum-group element micronuggets and refertilization process in Lherz orogenic peridotite (northeastern Pyrenees, France). Earth and Planetary Science Letters, 289, 298-310. doi: 16/j.epsl.2009.11.017.

Luguet, A., Alard, O., Lorand, J., Pearson, N., Ryan, C., and O'Reilly, S., 2001. Laserablation microprobe (LAM)-ICPMS unravels the highly siderophile element geochemistry of the oceanic mantle. Earth and Planetary Science Letters, 189, 285-294. doi: 10.1016/S0012-821X(01)00357-0.

Maier, W., 2005. Platinum-group element (PGE) deposits and occurrences: Mineralization styles, genetic concepts, and exploration criteria. Journal of African Earth Sciences, 41, 
165-191. doi: 10.1016/j.jafrearsci.2005.03.004.

Maier, W. D., Barnes, S. J., Campbell, I. H., Fiorentini, M. L., Peltonen, P., Barnes, S., and Smithies, R. H., 2009. Progressive mixing of meteoritic veneer into the early Earth's deep mantle. Nature, 460, 620-623. doi: 10.1038/nature08205.

Mann, J. L. and Kelly, W. R., 2005. Measurement of sulfur isotope composition $\delta^{34} \mathrm{~S}$ by multiple-collector thermal ionization mass spectrometry using a ${ }^{33} \mathrm{~S}-{ }^{36} \mathrm{~S}$ double spike. Rapid Communications in Mass Spectrometry, 19, 3429-3441. doi: 10.1002/rcm.2213.

Mann, U., Frost, D. J., Rubie, D. C., Becker, H., and Audétat, A., 2012. Partitioning of $\mathrm{Ru}, \mathrm{Rh}, \mathrm{Pd}, \mathrm{Re}$, Ir and Pt between liquid metal and silicate at high pressures and high temperatures - implications for the origin of highly siderophile element concentrations in the Earth's mantle. Geochimica et Cosmochimica Acta, 84, 593-613. doi: 10.1016/j. gca.2012.01.026.

Marcantonio, F., Reisberg, L., Zindler, A., Wyman, D., and Hulbert, L., 1994. An isotopic study of the Ni-Cu-PGE-rich Wellgreen intrusion of the Wrangellia Terrane: Evidence for hydrothermal mobilization of rhenium and osmium. Geochimica et Cosmochimica Acta, 58, 1007-1018. doi: 10.1016/0016-7037(94)90522-3.

Maréchal, C. N., Télouk, P., and Albarède, F., 1999. Precise analysis of copper and zinc isotopic compositions by plasma-source mass spectrometry. Chemical Geology, 156, 251-273. doi: 10.1016/S0009-2541(98)00191-0.

Markey, R., Hannah, J. L., Morgan, J. W., and Stein, H. J., 2003. A double spike for osmium analysis of highly radiogenic samples. Chemical Geology, 200, 395-406. doi: 10.1016/S0009-2541(03)00197-9.

Masarik, J., 1997. Contribution of neutron-capture reactions to observed tungsten isotopic ratios. Earth and Planetary Science Letters, 152, 181-185. doi: 10.1016/S0012-821X(97) 00151-9.

McCallum, M. E., Loucks, R. R., Carlson, R. R., Cooley, E. F., and Doerge, T. A., 1976. Platinum metals associated with hydrothermal copper ores of the New Rambler Mine, Medicine Bow Mountains, Wyoming. Economic Geology, 71, 1429-1450. doi: 10.2113/gsecongeo.71.7.1429.

McCoy, T., Keil, K., Clayton, R., Mayeda, T., Bogard, D., Garrison, D., and Wieler, R., 1997a. A petrologic and isotopic study of lodranites: Evidence for early formation as partial melt residues from heterogeneous precursors. Geochimica et Cosmochimica Acta, 61, 623-637. doi: 10.1016/S0016-7037(96)00359-6.

McCoy, T., Keil, K., Muenow, D., and Wilson, L., 1997b. Partial melting and melt migration in the acapulcoite-lodranite parent body. Geochimica et Cosmochimica Acta, 
61, 639-650. doi: 10.1016/S0016-7037(96)00365-1.

McDonough, W., 2003. Compositional model for the Earth's core. In Treatise on Geochemistry, Heinrich D. Holland and Karl K. Turekian (Eds.) 2 547-568. Pergamon, Oxford.

McDonough, W. and Sun, S.-s., 1995. The composition of the Earth. Chemical Geology, 120, 223-253. doi: 10.1016/0009-2541(94)00140-4.

McDonough, W. F., 2011. Meteoritic clues point chromium toward Earth's core. Science, 331, 1397-1398. doi: 10.1126/science.1203353.

McKeegan, K. D. and Davis, A. M., 2003. Early solar system chronology. In Treatise on Geochemistry, Holland, H. D. and Turekian, K. K. (Eds.) 1 431-460. Pergamon.

McSween, H. Y., 1979. Are carbonaceous chondrites primitive or processed? - a review. Reviews of Geophysics and Space Physics, 17, 1059-1078. doi: 10.1029/RG017i005p01059.

Meisel, T., Walker, R. J., and Morgan, J. W., 1996. The osmium isotopic composition of the Earth's primitive upper mantle. Nature, 383, 517-520. doi: 10.1038/383517a0.

Meisel, T. and Moser, J., 2004. Reference materials for geochemical PGE analysis: new analytical data for $\mathrm{Ru}, \mathrm{Rh}, \mathrm{Pd}, \mathrm{Os}$, Ir, Pt and Re by isotope dilution ICP-MS in 11 geological reference materials. Chemical Geology, 208, 319-338. doi: 10.1016/j.chemgeo. 2004.04.019.

Michlovich, E. S., Vogt, S., Masarik, J., Reedy, R. C., Elmore, D., and Lipschutz, M. E., 1994. Aluminum-26, ${ }^{10} \mathrm{Be}$, and ${ }^{36} \mathrm{Cl}$ depth profiles in the Canyon Diablo iron meteorites. Journal of Geophysical Research: Planets, 99, 23187-23194. doi: 10.1029/94JE02267.

Millet, M.-A., Baker, J. A., and Payne, C. E., 2012. Ultra-precise stable Fe isotope measurements by high resolution multiple-collector inductively coupled plasma mass spectrometry with a ${ }^{57} \mathrm{Fe}-{ }^{58} \mathrm{Fe}$ double spike. Chemical Geology, 304-305, 18-25. doi: 10.1016/j.chemgeo.2012.01.021.

Mitchell, R. H. and Keays, R. R., 1981. Abundance and distribution of gold, palladium and iridium in some spinel and garnet lherzolites: implications for the nature and origin of precious metal-rich intergranular components in the upper mantle. Geochimica et Cosmochimica Acta, 45, 2425-2442. doi: 10.1016/0016-7037(81)90096-X.

Mittlefehldt, D., 2007. Achondrites. In Treatise on Geochemistry, Holland, Heinrich D. and Turekian, Karl K. (Eds.) 1-40. Pergamon, Oxford.

Molini-Velsko, C., Mayeda, T. K., and Clayton, R. N., 1986. Isotopic composition of silicon in meteorites. Geochimica et Cosmochimica Acta, 50, 2719-2726. doi: 10.1016/ 0016-7037(86)90221-8. 
Mondal, S., 2011. Platinum group element (PGE) geochemistry to understand the chemical evolution of the Earth's mantle. Journal of the Geological Society of India, 77, 295-302. doi: 10.1007/s12594-011-0039-y.

Morbidelli, A., Chambers, J., Lunine, J. I., Petit, J. M., Robert, F., Valsecchi, G. B., and Cyr, K. E., 2000. Source regions and timescales for the delivery of water to the Earth. Meteoritics $\&$ Planetary Science, 35, 1309-1320. doi: 10.1111/j.1945-5100.2000. tb01518.x.

Morbidelli, A., Petit, J.-M., Gladman, B., and Chambers, J., 2001. A plausible cause of the late heavy bombardment. Meteoritics $\&$ Planetary Science, 36, 371-380.

Morgan, J. W., 1986. Ultramafic xenoliths: Clues to Earth's late accretionary history. Journal of Geophysical Research: Solid Earth, 91, 12375-12387. doi: 10.1029/ JB091iB12p12375.

Morgan, J. W., Walker, R. J., Horan, M. F., Beary, E. S., and Naldrett, A. J., 2002. ${ }^{190} \mathrm{Pt}-{ }^{186}$ Os and ${ }^{187} \mathrm{Re}-{ }^{187}$ Os systematics of the Sudbury Igneous Complex, Ontario. Geochimica et Cosmochimica Acta, 66, 273-290. doi: 10.1016/S0016-7037(01)00768-2.

Mountain, B. W. and Wood, S. A., 1988. Chemical controls on the solubility, transport and deposition of platinum and palladium in hydrothermal solutions; a thermodynamic approach. Economic Geology, 83, 492-510. doi: 10.2113/gsecongeo.83.3.492.

Moynier, F., Albarède, F., and Herzog, G., 2006. Isotopic composition of zinc, copper, and iron in lunar samples. Geochimica et Cosmochimica Acta, 70, 6103-6117. doi: 10.1016/j.gca.2006.02.030.

Moynier, F., Blichert-Toft, J., Telouk, P., Luck, J.-M., and Albarède, F., 2007. Comparative stable isotope geochemistry of $\mathrm{Ni}, \mathrm{Cu}, \mathrm{Zn}$, and $\mathrm{Fe}$ in chondrites and iron meteorites. Geochimica et Cosmochimica Acta, 71, 4365-4379. doi: 10.1016/j.gca.2007.06.049.

Moynier, F., Yin, Q.-Z., and Schauble, E., 2011. Isotopic evidence of Cr partitioning into earth's core. Science, 331, 1417-1420. doi: 10.1126/science.1199597.

Mughabghab, S. F., 2003. Thermal neutron capture cross sections resonance integrals and g-factors. International Atomic Energy Agency.

Mullane, E., Russell, S. S., and Gounelle, M., 2002. Iron isotope fractionation within a differentiated asteroidal sample suite. Meteoritics and Planetary Science Supplement, 37,105 .

Mullane, E., Russell, S. S., Gounelle, M., and Mason, T. F. D., 2003a. Iron isotope composition of Allende and Chainpur chondrules: Effects of equilibration and thermal history. In Lunar and Planetary Institute Science Conference Abstracts, 34, 1027. 
Mullane, E., Russell, S. S., Gounelle, M., and Mason, T. F. D., 2003b. Iron isotope composition of allende matrix, CAIs and chondrules. Meteoritics and Planetary Science Supplement, 38, 5117.

Mullane, E., Russell, S. S., Gounelle, M., Mason, T. F. D., Weiss, D., and Coles, B., 2003c. Magmatic \& impact processing on the HED parent body: Effects on iron isotope signatures. Geochimica et Cosmochimica Acta Supplement, 67, 311.

Murthy, V. R., 1991. Early differentiation of the Earth and the problem of mantle siderophile elements: A new approach. Science, 253, 303-306. doi: 10.1126/science. 253.5017.303.

Naldrett, A. J. and Duke, J. M., 1980. Platinum metals magmatic sulfide ores. Science, 208, 1417-1424. doi: 10.1126/science.208.4451.1417.

Newsom, H. E. and Sims, K. W. W., 1991. Core formation during early accretion of the Earth. Science, 252, 926 -933. doi: 10.1126/science.252.5008.926.

Nier, A. O. and Gulbransen, E. A., 1939. Variations in the relative abundance of the carbon isotopes. Journal of the American Chemical Society, 61, 697-698.

Nisbet, E., Cheadle, M., Arndt, N., and Bickle, M., 1993. Constraining the potential temperature of the Archaean mantle: A review of the evidence from komatiites. Lithos, 30, 291-307. doi: 10.1016/0024-4937(93)90042-B.

Nisbet, E. G. and Walker, D., 1982. Komatiites and the structure of the Archaean mantle. Earth and Planetary Science Letters, 60, 105-113. doi: 10.1016/0012-821X(82)90025-5.

Nittler, L. R. and Dauphas, N., 2006. Meteorites and the chemical evolution of the Milky Way. Meteorites and the Early Solar System II, 943, 127-146.

Norman, M. D., 2009. The lunar cataclysm: Reality or "Mythconception"? Elements, 5, 23-28. doi: 10.2113/gselements.5.1.23.

Ohtani, E., Yurimoto, H., and Seto, S., 1997. Element partitioning between metallic liquid, silicate liquid, and lower-mantle minerals: implications for core formation of the Earth. Physics of The Earth and Planetary Interiors, 100, 97-114. doi: 10.1016/ S0031-9201(96)03234-7.

Pahlevan, K. and Stevenson, D. J., 2007. Equilibration in the aftermath of the lunarforming giant impact. Earth and Planetary Science Letters, 262, 438-449. doi: 10. 1016/j.epsl.2007.07.055

Palme, H. and O'Neill, H. S. C., 2007. Cosmochemical estimates of mantle compositions. In Treatise on Geochemistry, Heinrich D. Holland and Karl K. Turekian (Eds.) 1-38. Pergamon, Oxford. 
Paton, C., Hellstrom, J., Paul, B., Woodhead, J., and Hergt, J., 2011. Iolite: Freeware for the visualisation and processing of mass spectrometric data. Journal of Analytical Atomic Spectrometry, 26, 2508-2518. doi: 10.1039/c1ja10172b.

Patterson, C., 1956. Age of meteorites and the Earth. Geochimica et Cosmochimica Acta, 10, 230-237. doi: 10.1016/0016-7037(56)90036-9.

Pattou, L., Lorand, J. P., and Gros, M., 1996. Non-chondritic platinum-group element ratios in the Earth's mantle. Nature, 379, 712-715. doi: 10.1038/379712a0.

Pearson, D. G. and Woodland, S. J., 2000. Solvent extraction/anion exchange separation and determination of PGEs ( $\mathrm{Os}, \mathrm{Ir}, \mathrm{Pt}, \mathrm{Pd}, \mathrm{Ru}$ ) and Re-Os isotopes in geological samples by isotope dilution ICP-MS. Chemical Geology, 165, 87-107. doi: 10.1016/S0009-2541(99)00161-8.

Pearson, D., Irvine, G., Ionov, D., Boyd, F., and Dreibus, G., 2004. Re-Os isotope systematics and platinum group element fractionation during mantle melt extraction: a study of massif and xenolith peridotite suites. Chemical Geology, 208, 29-59. doi: 10.1016/j.chemgeo.2004.04.005.

Petaev, M. I. and Jacobsen, S. B., 2004. Differentiation of metal-rich meteoritic parent bodies: I. measurements of PGEs, Re, Mo, W, and $\mathrm{Au}$ in meteoritic Fe-Ni metals. Meteoritics $\&$ Planetary Science, 39, 1685-1697. doi: 10.1111/j.1945-5100.2004.tb00066. $\mathrm{x}$.

Poirier, J.-P., 1994. Light elements in the Earth's outer core: A critical review. Physics of the Earth and Planetary Interiors, 85, 319-337. doi: 10.1016/0031-9201(94)90120-1.

Poirier, J.-P. and le Mouël, J.-L., 1992. Does infiltration of core material into the lower mantle affect the observed geomagnetic field? Physics of the Earth and Planetary Interiors, 73, 29-37. doi: 10.1016/0031-9201(92)90105-5.

Poitrasson, F., Halliday, A. N., Lee, D.-C., Levasseur, S., and Teutsch, N., 2004. Iron isotope differences between Earth, Moon, Mars and Vesta as possible records of contrasted accretion mechanisms. Earth and Planetary Science Letters, 223, 253-266. doi: 10.1016/j.epsl.2004.04.032.

Poitrasson, F., Levasseur, S., and Teutsch, N., 2005. Significance of iron isotope mineral fractionation in pallasites and iron meteorites for the core-mantle differentiation of terrestrial planets. Earth and Planetary Science Letters, 234, 151-164. doi: 10.1016/j. epsl.2005.02.010.

Polyakov, V. B., 2009. Equilibrium iron isotope fractionation at core-mantle boundary conditions. Science, 323, 912-914. doi: 10.1126/science.1166329.

Powell, R., Woodhead, J., and Hergt, J., 1998. Uncertainties on lead isotope analyses: 
deconvolution in the double-spike method. Chemical Geology, 148, 95-104. doi: 10. 1016/S0009-2541(98)00023-0.

Puchtel, I. and Humayun, M., 2000. Platinum group elements in Kostomuksha komatiites and basalts: implications for oceanic crust recycling and core-mantle interaction. Geochimica et Cosmochimica Acta, 64, 4227-4242. doi: 10.1016/S0016-7037(00)00492-0.

Puchtel, I. S. and Humayun, M., 2001. Platinum group element fractionation in a komatiitic basalt lava lake. Geochimica et Cosmochimica Acta, 65, 2979-2993. doi: 10.1016/S0016-7037(01)00642-1.

Qin, L., Dauphas, N., Wadhwa, M., Masarik, J., and Janney, P. E., 2008. Rapid accretion and differentiation of iron meteorite parent bodies inferred from ${ }^{182} \mathrm{Hf}-{ }^{182} \mathrm{~W}$ chronometry and thermal modeling. Earth and Planetary Science Letters, 273, 94-104. doi: 10.1016/j.epsl.2008.06.018.

Quitté, G., Halliday, A. N., Markowski, A., Bourdon, B., Meyer, B., Zanda, B., Latkoczy, C., and Günther, D., 2006a. Ni isotopes in the early solar system: an overview. $A G U$ Fall Meeting Abstracts, -1, 0548.

Quitté, G., Meier, M., Latkoczy, C., Halliday, A., and Günther, D., 2006b. Nickel isotopes in iron meteorites-nucleosynthetic anomalies in sulphides with no effects in metals and no trace of ${ }^{60} \mathrm{Fe}$. Earth and Planetary Science Letters, 242, 16-25. doi: 10.1016/j.epsl. 2005.11.053.

Ragsdale, S. W., 2007. Nickel and the carbon cycle. Journal of Inorganic Biochemistry, 101, 1657-1666. doi: 10.1016/j.jinorgbio.2007.07.014.

Rankenburg, K., Humayun, M., Brandon, A., and Herrin, J., 2008. Highly siderophile elements in ureilites. Geochimica et Cosmochimica Acta, 72, 4642-4659. doi: 16/j.gca. 2008.07.003.

Ravizza, G. and Pyle, D., 1997. PGE and Os isotopic analyses of single sample aliquots with NiS fire assay preconcentration. Chemical Geology, 141, 251-268. doi: 16/S0009-2541(97)00091-0.

Regelous, M., Elliott, T., and Coath, C. D., 2008. Nickel isotope heterogeneity in the early Solar System. Earth and Planetary Science Letters, 272, 330-338. doi: 10.1016/j.epsl. 2008.05.001.

Rehkämper, M. and Halliday, A. N., 1997. Development and application of new ionexchange techniques for the separation of the platinum group and other siderophile elements from geological samples. Talanta, 44, 663-672. doi: DOI:10.1016/S0039-9140(96) 02100-5

Rehkämper, M., Halliday, A. N., Alt, J., Fitton, J. G., Zipfel, J., and Takazawa, E., 
1999a. Non-chondritic platinum-group element ratios in oceanic mantle lithosphere: petrogenetic signature of melt percolation? Earth and Planetary Science Letters, 172, $65-81$.

Rehkämper, M., Halliday, A. N., Barfod, D., Fitton, J. G., and Dawson, J. B., 1997. Platinum-Group element abundance patterns in different mantle environments. Science, 278, 1595-1598. doi: 10.1126/science.278.5343.1595.

Rehkämper, M., Halliday, A. N., Barfod, D., Fitton, J. G., and Dawson, J. B., 1997. Platinum-group element abundance patterns in different mantle environments. Science, 278, 1595 -1598. doi: 10.1126/science.278.5343.1595.

Rehkämper, M., Halliday, A., Fitton, J., Lee, D.-C., Wieneke, M., and Arndt, N., 1999b. $\mathrm{Ir}, \mathrm{Ru}, \mathrm{Pt}$, and $\mathrm{Pd}$ in basalts and komatiites: new constraints for the geochemical behavior of the platinum-group elements in the mantle. Geochimica et Cosmochimica Acta, 63, 3915-3934. doi: 10.1016/S0016-7037(99)00219-7.

Richter, F., Dauphas, N., and Teng, F., 2009. Non-traditional fractionation of nontraditional isotopes: Evaporation, chemical diffusion and soret diffusion. Chemical Geology, 258, 92-103. doi: 10.1016/j.chemgeo.2008.06.011.

Richter, F. M., 1985. Models for the archean thermal regime. Earth and Planetary Science Letters, 73, 350-360. doi: 10.1016/0012-821X(85)90083-4.

Righter, K., 2011. Prediction of metal-silicate partition coefficients for siderophile elements: An update and assessment of PT conditions for metal-silicate equilibrium during accretion of the Earth. Earth and Planetary Science Letters, 304, 158-167. doi: 10.1016/j.epsl.2011.01.028.

Righter, K. and Drake, M., 2003. Partition coefficients at high pressure and temperature. In Treatise on Geochemistry, Heinrich D. Holland and Karl K. Turekian (Eds.) 425-449. Pergamon, Oxford.

Righter, K., Humayun, M., and Danielson, L., 2008. Partitioning of palladium at high pressures and temperatures during core formation. Nature Geoscience, 1, 321-323. doi: $10.1038 /$ ngeo180.

Righter, K., King, C., Danielson, L., Pando, K., and Lee, C., 2011. Experimental determination of the metal/silicate partition coefficient of germanium: Implications for core and mantle differentiation. Earth and Planetary Science Letters, 304, 379-388. doi: 10.1016/j.epsl.2011.02.015.

Righter, K., 2003. Metal-silicate partitioning of siderophile elements and core formation in the early Earth. Annual Review of Earth and Planetary Sciences, 31, 135-174. doi: 10.1146/annurev.earth.31.100901.145451. 
Righter, K. and Drake, M. J., 1996. Core formation in Earth's Moon, Mars, and Vesta. Icarus, 124, 513-529. doi: 10.1006/icar.1996.0227.

Righter, K. and Drake, M. J., 1997. Metal-silicate equilibrium in a homogeneously accreting Earth: new results for Re. Earth and Planetary Science Letters, 146, 541-553. doi: 10.1016/S0012-821X(96)00243-9.

Righter, K. and Drake, M. J., 1999. Effect of water on metal-silicate partitioning of siderophile elements: a high pressure and temperature terrestrial magma ocean and core formation. Earth and Planetary Science Letters, 171, 383-399. doi: 10.1016/ S0012-821X(99)00156-9.

Ringwood, A. E., Kato, T., Hibberson, W., and Ware, N., 1990. High-pressure geochemistry of $\mathrm{Cr}, \mathrm{V}$ and $\mathrm{Mn}$ and implications for the origin of the Moon. Nature, 347, 174-176. doi: $10.1038 / 347174 a 0$.

Ringwood, A., 1966. Chemical evolution of the terrestrial planets. Geochimica et Cosmochimica Acta, 30, 41-104. doi: 10.1016/0016-7037(66)90090-1.

Ringwood, A. E., 1977. Composition of the core and implications for the origin of the Earth. Geochemical Journal, 111-135.

Ripperger, S. and Rehkämper, M., 2007. Precise determination of cadmium isotope fractionation in seawater by double spike MC-ICPMS. Geochimica et Cosmochimica Acta, 71, 631-642. doi: 10.1016/j.gca.2006.10.005.

Roberts, J., Thomson, A., and Cohn, W. E., 1979. The mechanism of action of antitumor platinum compounds. In Progress in Nucleic Acid Research and Molecular Biology 22 71-133. Academic Press.

Rosman, K. J. R. and Taylor, P. D. P., 1998. Isotopic compositions of the elements 1997. Journal of Physical and Chemical Reference Data, 27, 1275-1287.

Rosman, K., 1972. A survey of the isotopic and elemental abundance of zinc. Geochimica et Cosmochimica Acta, 36, 801-819. doi: 10.1016/0016-7037(72)90089-0.

Rouxel, O., Dobbek, N., Ludden, J., and Fouquet, Y., 2003. Iron isotope fractionation during oceanic crust alteration. Chemical Geology, 202, 155-182. doi: 10.1016/j.chemgeo.2003.08.011.

Rowell, W. F. and Edgar, A. D., 1986. Platinum-group element mineralization in a hydrothermal $\mathrm{Cu}-\mathrm{Ni}$ sulfide occurrence, Rathbun Lake, northeastern Ontario. Economic Geology, 81, 1272-1277. doi: 10.2113/gsecongeo.81.5.1272.

Rubie, D. C., Frost, D. J., Mann, U., Asahara, Y., Nimmo, F., Tsuno, K., Kegler, P., Holzheid, A., and Palme, H., 2011. Heterogeneous accretion, composition and 
core-mantle differentiation of the Earth. Earth and Planetary Science Letters, 301, 31-42. doi: 10.1016/j.epsl.2010.11.030.

Rubin, A. E., 1989. Size-frequency distributions of chondrules in CO3 chondrites. Meteoritics, 24, 179-189. doi: 10.1111/j.1945-5100.1989.tb00960.x.

Rubin, A. E., 2007. Petrogenesis of acapulcoites and lodranites: A shock-melting model. Geochimica et Cosmochimica Acta, 71, 2383-2401. doi: 10.1016/j.gca.2007.02.010.

Rubin, A. E. and Mittlefehldt, D. W., 1993. Evolutionary history of the mesosiderite asteroid: A chronologic and petrologic synthesis. Icarus, 101, 201-212. doi: 10.1006/ icar.1993.1018.

Rubin, A. E., Scott, E. R., and Keil, K., 1997. Shock metamorphism of enstatite chondrites. Geochimica et Cosmochimica Acta, 61, 847-858. doi: 10.1016/S0016-7037(96) 00364-X.

Rubin, A. E., Kallemeyn, G. W., Wasson, J. T., Clayton, R. N., Mayeda, T. K., Grady, M., Verchovsky, A. B., Eugster, O., and Lorenzetti, S., 2003. Formation of metal and silicate globules in gujba: a new bencubbin-like meteorite fall. Geochimica et Cosmochimica Acta, 67, 3283-3298. doi: 10.1016/S0016-7037(03)00098-X.

Rudge, J. F., Reynolds, B. C., and Bourdon, B., 2009. The double spike toolbox. Chemical Geology, 265, 420-431. doi: 10.1016/j.chemgeo.2009.05.010.

Rudge, J. F., Kleine, T., and Bourdon, B., 2010. Broad bounds on Earth's accretion and core formation constrained by geochemical models. Nature Geosci, 3, 439-443. doi: $10.1038 /$ ngeo872.

Russell, R. D., 1971. The systematics of double spiking. Journal of Geophysical Research, 76, 4949-4955. doi: 197110.1029/JB076i020p04949.

Savage, P. S. and Moynier, F., 2013. Silicon isotopic variation in enstatite meteorites: Clues to their origin and Earth-forming materials. Earth and Planetary Science Letters, 361, 487-496. doi: 10.1016/j.epsl.2012.11.016.

Savage, P. S., Georg, R. B., Williams, H. M., Burton, K. W., and Halliday, A. N., 2011. Silicon isotope fractionation during magmatic differentiation. Geochimica et Cosmochimica Acta, 75, 6124-6139. doi: 10.1016/j.gca.2011.07.043.

Savard, D., Barnes, S., and Meisel, T., 2010. Comparison between nickel-sulfur fire assay Te co-precipitation and isotope dilution with high-pressure asher acid digestion for the determination of platinum-group elements, rhenium and gold. Geostandards and Geoanalytical Research, 34, 281-291. doi: 10.1111/j.1751-908X.2010.00090.x.

Schauble, E. A., Tonui, E., Georg, R. B., Young, E. D., and Halliday, A. N., 2007. Estimat- 
ing magnesium and silicon isotope fractionation with first-principles lattice dynamics. In Geochimica et Cosmochimica Acta, 71, A884-A884.

Scherer, P. and Schultz, L., 2000. Noble gas record, collisional history, and pairing of CV, CO, CK, and other carbonaceous chondrites. Meteoritics \&3 Planetary Science, 35, 145-153. doi: 10.1111/j.1945-5100.2000.tb01981.x.

Scherstén, A., Elliott, T., Hawkesworth, C., Russell, S., and Masarik, J., 2006. Hf-W evidence for rapid differentiation of iron meteorite parent bodies. Earth and Planetary Science Letters, 241, 530-542. doi: 10.1016/j.epsl.2005.11.025.

Schmitt, A. D., Galer, S. J. G., and Abouchami, W., 2009. High-precision cadmium stable isotope measurements by double spike thermal ionisation mass spectrometry. Journal of Analytical Atomic Spectrometry, 24, 1079-1088.

Schmitt, W., Palme, H., and Wänke, H., 1989. Experimental determination of metal/silicate partition coefficients for $\mathrm{P}, \mathrm{Co}, \mathrm{Ni}, \mathrm{Cu}, \mathrm{Ga}, \mathrm{Ge}, \mathrm{Mo}$, and $\mathrm{W}$ and some implications for the early evolution of the Earth. Geochimica et Cosmochimica Acta, 53, 173-185. doi: 10.1016/0016-7037(89)90284-6.

Schoenberg, R., Zink, S., Staubwasser, M., and von Blanckenburg, F., 2008. The stable Cr isotope inventory of solid Earth reservoirs determined by double spike MC-ICP-MS. Chemical Geology, 249, 294-306. doi: 10.1016/j.chemgeo.2008.01.009.

Schönbächler, M., Carlson, R. W., Horan, M. F., Mock, T. D., and Hauri, E. H., 2010. Heterogeneous accretion and the moderately volatile element budget of Earth. Science, 328, 884-887. doi: 10.1126/science.1186239.

Scott, E. and Krot, A., 2007. Chondrites and their components. In Treatise on Geochemistry, Holland, H. D. and Turekian, K. K. (Eds.) 1-72. Pergamon, Oxford.

Sears, D. W., Kallemeyn, G. W., and Wasson, J. T., 1982. The compositional classification of chondrites: II the enstatite chondrite groups. Geochimica et Cosmochimica Acta, 46, 597-608. doi: 10.1016/0016-7037(82)90161-2.

Shahar, A. and Young, E. D., 2007. Astrophysics of CAI formation as revealed by silicon isotope LA-MC-ICPMS of an igneous CAI. Earth and Planetary Science Letters, 257, 497-510. doi: 10.1016/j.epsl.2007.03.012.

Shahar, A., Ziegler, K., Young, E. D., Ricolleau, A., Schauble, E. A., and Fei, Y., 2009. Experimentally determined Si isotope fractionation between silicate and Fe metal and implications for Earth's core formation. Earth and Planetary Science Letters, 288, 228-234. doi: 10.1016/j.epsl.2009.09.025.

Shahar, A., Hillgren, V. J., Young, E. D., Fei, Y., Macris, C. A., and Deng, L., 2011. High-temperature Si isotope fractionation between iron metal and silicate. Geochimica 
et Cosmochimica Acta, 75, 7688-7697. doi: 10.1016/j.gca.2011.09.038.

Shirey, S. B. and Walker, R. J., 1998. The Re-Os isotope system in cosmochemistry and high-temperature geochemistry. Annual Review of Earth and Planetary Sciences, 26, $423-500$.

Siebert, C., Nägler, T. F., and Kramers, J. D., 2001. Determination of molybdenum isotope fractionation by double-spike multicollector inductively coupled plasma mass spectrometry. Geochemistry Geophysics Geosystems, 2, 16 PP. doi: 200110.1029/ 2000GC000124[Citation].

Snow, J. E. and Schmidt, G., 1998. Constraints on Earth accretion deduced from noble metals in the oceanic mantle. Nature, 391, 166-169. doi: 10.1038/34396.

Steele, R. C., Elliott, T., Coath, C. D., and Regelous, M., 2011. Confirmation of massindependent $\mathrm{Ni}$ isotopic variability in iron meteorites. Geochimica et Cosmochimica Acta, 75, 7906-7925. doi: 10.1016/j.gca.2011.08.030.

Stevenson, D. J., 1987. Origin of the Moon-The Collision Hypothesis. Annual Review of Earth and Planetary Sciences, 15, 271-315. doi: 10.1146/annurev.ea.15.050187.001415.

Stevenson, D. J., 1990. Fluid dynamics of core formation. Origin of the Earth, -1, 231-249.

Tagle, R. and Berlin, J., 2008. A database of chondrite analyses including platinum group elements, $\mathrm{Ni}, \mathrm{Co}, \mathrm{Au}$, and $\mathrm{Cr}$ : Implications for the identification of chondritic projectiles. Meteoritics \& Planetary Science, 43, 541-559. doi: 10.1111/j.1945-5100.2008.tb00671.x.

Takazawa, E., Frey, F. A., Shimizu, N., Obata, M., and Bodinier, J. L., 1992. Geochemical evidence for melt migration and reaction in the upper mantle. Nature, 359, 55-58. doi: $10.1038 / 359055 \mathrm{a} 0$.

Takazawa, E., Frey, F., Shimizu, N., and Obata, M., 1996. Evolution of the Horoman Peridotite (Hokkaido, Japan): Implications from pyroxene compositions. Chemical Geology, 134, 3-26. doi: 10.1016/S0009-2541(96)00083-6.

Takazawa, E., Frey, F. A., Shimizu, N., Saal, A., and Obata, M., 1999. Polybaric petrogenesis of mafic layers in the Horoman Peridotite Complex, Japan. Journal of Petrology, 40, 1827-1851. doi: 10.1093/petroj/40.12.1827.

Takazawa, E., Frey, F., Shimizu, N., and Obata, M., 2000. Whole rock compositional variations in an upper mantle peridotite (Horoman, Hokkaido, Japan): are they consistent with a partial melting process? Geochimica et Cosmochimica Acta, 64, 695-716. doi: 10.1016/S0016-7037(99)00346-4.

Taylor, P. D. P., Valkiers, S., De Bièvre, P., Flegel, U., and Kruck, T., 1994. In Proceedings of the Second Alfred O. Nier Symposium on Inorganic Mass Spectrometry: Durango, 
Colorado, 1994 May 9-12 90-94.

Taylor, S. R., 2001. Solar System Evolution: A New Perspective. Cambridge University Press, 2nd edition. ISBN 9780521641302.

Tera, F., Papanastassiou, D. A., and Wasserburg, G. J., 1974. Isotopic evidence for a terminal lunar cataclysm. Earth and Planetary Science Letters, 22, 1-21.

Tian, X., Emteborg, H., and Adams, F. C., 1999. Analytical performance of axial inductively coupled plasma time of flight mass spectrometry (ICP-TOFMS). Journal of Analytical Atomic Spectrometry, 14, 1807-1814. doi: 10.1039/A906762K.

Tonks, W. and Melosh, H. J., 1992. Core formation by giant impacts. Icarus, 100, 326-346.

Touboul, M., Kleine, T., Bourdon, B., Palme, H., and Wieler, R., 2007. Late formation and prolonged differentiation of the Moon inferred from $\mathrm{W}$ isotopes in lunar metals. Nature, 450, 1206-1209. doi: 10.1038/nature06428.

Turekian, K. K. and Clark, S. P., 1969. Inhomogeneous accumulation of the Earth from the primitive solar nebula. Earth and Planetary Science Letters, 6, 346-348.

Urey, H. C., 1947. The thermodynamic properties of isotopic substances. Journal of the Chemical Society (Resumed) 562-581. doi: 10.1039/JR9470000562.

Urey, H. C., 1948. Oxygen isotopes in nature and in the laboratory. Science, 108, 489-496.

Voshage, H. and Feldmann, H., 1979. Investigations on cosmic-ray-produced nuclides in iron meteorites, 3. Exposure ages, meteoroid sizes and sample depths determined by mass spectrometric analyses of potassium and rare gases. Earth and Planetary Science Letters, 45, 293-308. doi: 10.1016/0012-821X(79)90131-6.

Voshage, H., 1984. Investigations of cosmic-ray-produced nuclides in iron meteorites, 6. The Signer-Nier model and the history of the cosmic radiation. Earth and Planetary Science Letters, 71, 181-194. doi: 10.1016/0012-821X(84)90064-5.

Wade, J. and Wood, B. J., 2001. The Earth's 'missing' niobium may be in the core. Nature, 409, 75-78. doi: 10.1038/35051064.

Wade, J. and Wood, B., 2005. Core formation and the oxidation state of the Earth. Earth and Planetary Science Letters, 236, 78-95. doi: 10.1016/j.epsl.2005.05.017.

Wadhwa, M., 2007. Long-lived chronometers. In Treatise on Geochemistry, Heinrich D. Holland and Karl K. Turekian (Eds.) 1-25. Pergamon, Oxford.

Walker, D., Norby, L., and Jones, J. H., 1993. Superheating effects on metal-silicate partitioning of siderophile elements. Science, 262, 1858 -1861. doi: 10.1126/science.262. 5141.1858. 
Walker, R., 1997. Applications of the ${ }^{190} \mathrm{Pt}-{ }^{186} \mathrm{Os}$ isotope system to geochemistry and cosmochemistry. Geochimica et Cosmochimica Acta, 61, 4799-4807. doi: 10.1016/ S0016-7037(97)00270-6.

Walker, R. J., 2009. Highly siderophile elements in the Earth, Moon and Mars: Update and implications for planetary accretion and differentiation. Chemie der Erde Geochemistry, 69, 101-125. doi: 10.1016/j.chemer.2008.10.001.

Walker, R. J., Morgan, J. W., and Horan, M. F., 1995. Osmium-187 enrichment in some plumes: Evidence for core-mantle interaction? Science, 269, 819-822. doi: 10.1126/ science.269.5225.819.

Walker, R., Horan, M., Morgan, J., and Meisel, T., 2001. Osmium isotopic compositions of chondrites and Earth's primitive upper mantle: Constraints on the late veneer. In Lunar and Planetary Institute Science Conference Abstracts, 32, 1152.

Walker, R., Horan, M., Morgan, J., Becker, H., Grossman, J., and Rubin, A., 2002. Comparative ${ }^{187} \mathrm{Re}^{-{ }^{187}}$ Os systematics of chondrites: Implications regarding early solar system processes. Geochimica et Cosmochimica Acta, 66, 4187-4201. doi: 10.1016/ S0016-7037(02)01003-7.

Walker, R., Horan, M., Shearer, C., and Papike, J., 2004. Low abundances of highly siderophile elements in the lunar mantle: evidence for prolonged late accretion. Earth and Planetary Science Letters, 224, 399-413. doi: 10.1016/j.epsl.2004.05.036.

Walraven, F., Armstrong, R., and Kruger, F., 1990. A chronostratigraphic framework for the north-central Kaapvaal craton, the Bushveld Complex and the Vredefort structure. Tectonophysics, 171, 23-48. doi: 10.1016/0040-1951(90)90088-P.

Wang, Z. and Becker, H., 2013. Ratios of S, Se and Te in the silicate Earth require a volatile-rich late veneer. Nature, 499, 328-331. doi: 10.1038/nature12285.

Wänke, H. and Gold, T., 1981. Constitution of terrestrial planets [and discussion]. Philosophical Transactions of the Royal Society of London. Series A, Mathematical and Physical Sciences, 303, 287-302. doi: 10.1098/rsta.1981.0203.

Wänke, H., Dreibus, G., and Jagoutz, E., 1984. Mantle chemistry and accretion history of the Earth. In Archaean Geochemistry, Kröner, P. A., Hanson, P. G. N., and Goodwin, P. A. M. (Eds.) 1-24. Springer Berlin Heidelberg.

Wedepohl, K., 1995. The composition of the continental crust. Geochimica et Cosmochimica Acta, 59, 1217-1232. doi: 10.1016/0016-7037(95)00038-2.

Weisberg, M. K., McCoy, T. J., and Krot, A. N., 2006. Systematics and evaluation of meteorite classification. Meteorites and the early solar system II, 1, 19-52. 
Wetherill, G. W., 1975. Late heavy bombardment of the moon and terrestrial planets. In Lunar and Planetary Science Conference Proceedings, 6, 1539-1561.

Wetherill, G. W., 1985. Occurrence of giant impacts during the growth of the terrestrial planets. Science, 228, 877-879. doi: 10.1126/science.228.4701.877.

Weyer, S., Anbar, A., Brey, G., Munker, C., Mezger, K., and Woodland, A., 2005. Iron isotope fractionation during planetary differentiation. Earth and Planetary Science Letters, 240, 251-264. doi: 10.1016/j.epsl.2005.09.023.

Weyer, S., Anbar, A., Brey, G., Münker, C., Mezger, K., and Woodland, A., 2007. Feisotope fractionation during partial melting on Earth and the current view on the Feisotope budgets of the planets. Earth and Planetary Science Letters, 256, 638-646. doi: 10.1016/j.epsl.2007.01.038.

White, F. A., Collins, T. L., and Rourke, F. M., 1956. Search for possible naturally occurring isotopes of low abundance. Physical Review, 101, 1786-1791. doi: 10.1103/ PhysRev.101.1786.

Wiesli, R. A., Beard, B. L., Taylor, L. A., and Johnson, C. M., 2003. Space weathering processes on airless bodies: Fe isotope fractionation in the lunar regolith. Earth and Planetary Science Letters, 216, 457-465. doi: 10.1016/S0012-821X(03)00552-1.

Willbold, M., Elliott, T., and Moorbath, S., 2011. The tungsten isotopic composition of the Earth's mantle before the terminal bombardment. Nature, 477, 195-198. doi: $10.1038 /$ nature10399.

Williams, H. M., McCammon, C. A., Peslier, A. H., Halliday, A. N., Teutsch, N., Levasseur, S., and Burg, J.-P., 2004. Iron isotope fractionation and the oxygen fugacity of the mantle. Science, 304, 1656-1659. doi: 10.1126/science.1095679.

Williams, H., Markowski, A., Quitté, G., Halliday, A., Teutsch, N., and Levasseur, S., 2006. Fe isotope fractionation in iron meteorites: New insights into metal-sulphide segregation and planetary accretion. Earth and Planetary Science Letters, 250, 486500. doi: 10.1016/j.epsl.2006.08.013.

Williams, R., 2010. Uncertainty in measurement of isotope ratios by multi-collector mass spectrometry. IAEA-CN-184/168, Vienna, Austria. International Atomic Energy Agency.

Wittig, N., Humayun, M., Brandon, A., Huang, S., and Leya, I., 2013. Coupled W-Os-Pt isotope systematics in IVB iron meteorites: In situ neutron dosimetry for W isotope chronology. Earth and Planetary Science Letters, 361, 152-161. doi: 10.1016/j.epsl. 2012.10.013.

Wolff Briche, C., Held, A., Berglund, M., De Bièvre, P., and Taylor, P., 2002. Measurement 
of the isotopic composition and atomic weight of an isotopic reference material of platinum, IRMM-010. Analytica Chimica Acta, 460, 41-47. doi: 10.1016/S0003-2670(02) 00145-9.

Wood, B. J., Wade, J., and Kilburn, M. R., 2008. Core formation and the oxidation state of the Earth: Additional constraints from Nb, V and Cr partitioning. Geochimica et Cosmochimica Acta, 72, 1415-1426. doi: 10.1016/j.gca.2007.11.036.

Wood, J. A., 1964. The cooling rates and parent planets of several iron meteorites. Icarus, 3, 429-459. doi: 10.1016/0019-1035(64)90004-1.

Yang, J. and Goldstein, J. I., 2006. Metallographic cooling rates of the IIIAB iron meteorites. Geochimica et Cosmochimica Acta, 70, 3197-3215. doi: 10.1016/j.gca.2006.04. 007.

Yin, Q., Jacobsen, S. B., Yamashita, K., Blichert-Toft, J., Tèlouk, P., and Albarède, F., 2002. A short timescale for terrestrial planet formation from Hf-W chronometry of meteorites. Nature, 418, 949-952.

Young, E. D. and Galy, A., 2004. The isotope geochemistry and cosmochemistry of magnesium. Reviews in Mineralogy and Geochemistry, 55, 197-230. doi: 10.2138/gsrmg.55. 1.197 .

Zhu, X. K., Guo, Y., O'Nions, R. K., Young, E. D., and Ash, R. D., 2001. Isotopic homogeneity of iron in the early solar nebula. Nature, 412, 311-313. doi: 10.1038/ 35085525 .

Zhu, X. K., Guo, Y., Williams, R. J. P., O'nions, R. K., Matthews, A., Belshaw, N. S., Canters, G. W., De Waal, E. C., Weser, U., and Burgess, B. K., 2002. Mass fractionation processes of transition metal isotopes. Earth and Planetary Science Letters, 200, 47-62.

Zhu, X., O'Nions, R., Guo, Y., Belshaw, N., and Rickard, D., 2000. Determination of natural $\mathrm{Cu}$-isotope variation by plasma-source mass spectrometry: implications for use as geochemical tracers. Chemical Geology, 163, 139-149. doi: 10.1016/S0009-2541(99) 00076-5.

Ziegler, K., Young, E. D., Schauble, E. A., and Wasson, J. T., 2009. Silicon isotope fractionation between silicate and metal from an enstatite meteorite: Implications for Earth's core formation. Meteoritics and Planetary Science Supplement, 72, 5435.

Ziegler, K., Young, E. D., Schauble, E. A., and Wasson, J. T., 2010. Metal-silicate silicon isotope fractionation in enstatite meteorites and constraints on Earth's core formation. Earth and Planetary Science Letters, 295, 487-496. doi: 10.1016/j.epsl.2010.04.030. 
Appendices 


\section{Appendix A}

\section{Samples}

This appendix contains a list of meteorite samples analysed in this thesis with additional information from the literature and the meteorite databases. The list is separated into two parts, with the stony (i.e., chondrite, primitive achondrite and achondrite) meteorite samples in Table A.1, and the iron meteorites listed in Table A.2. The sample information is followed by followed by photographs of all of the meteorite specimens. References cited in this appendix are listed in the combined references section that immediately precedes this section. 


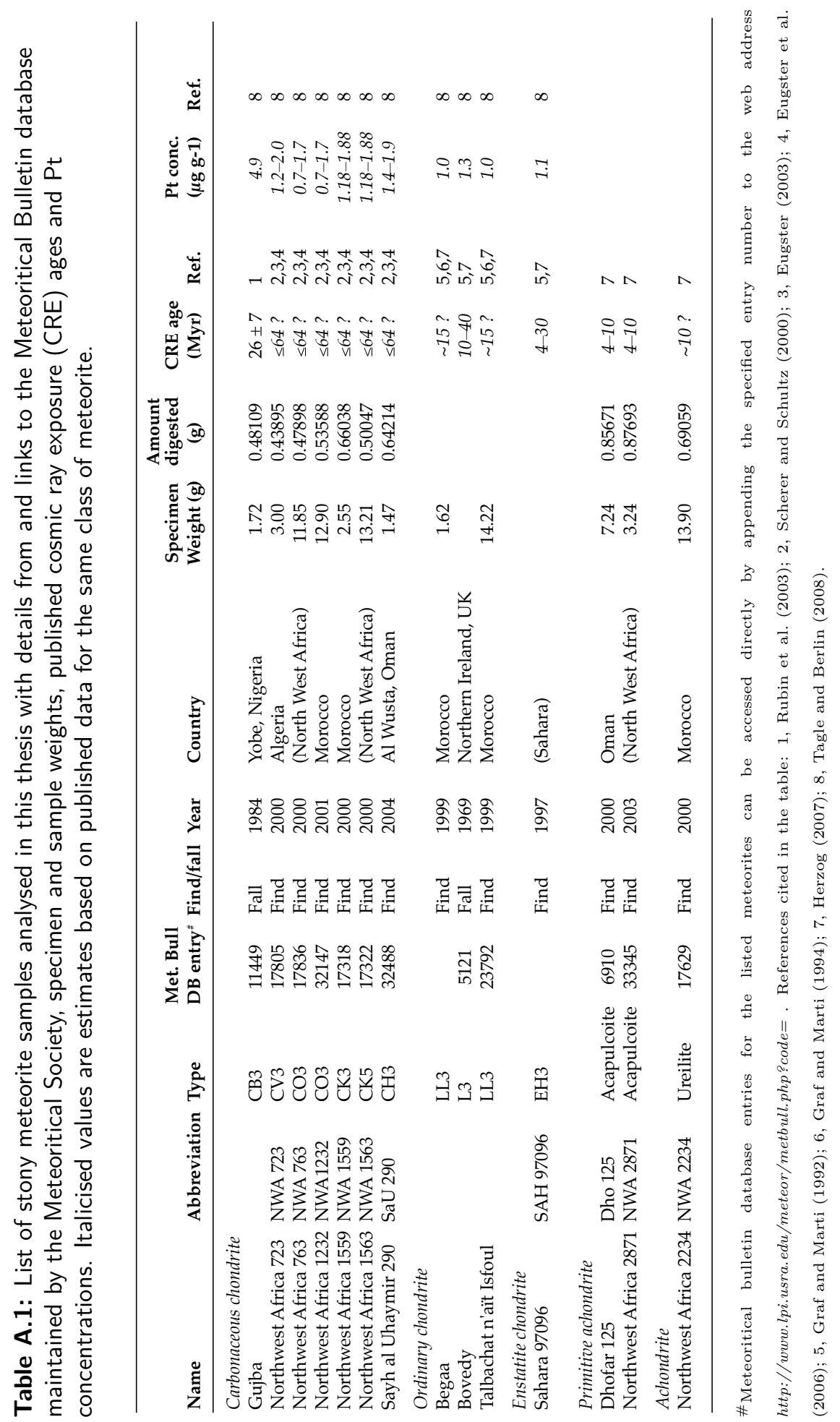




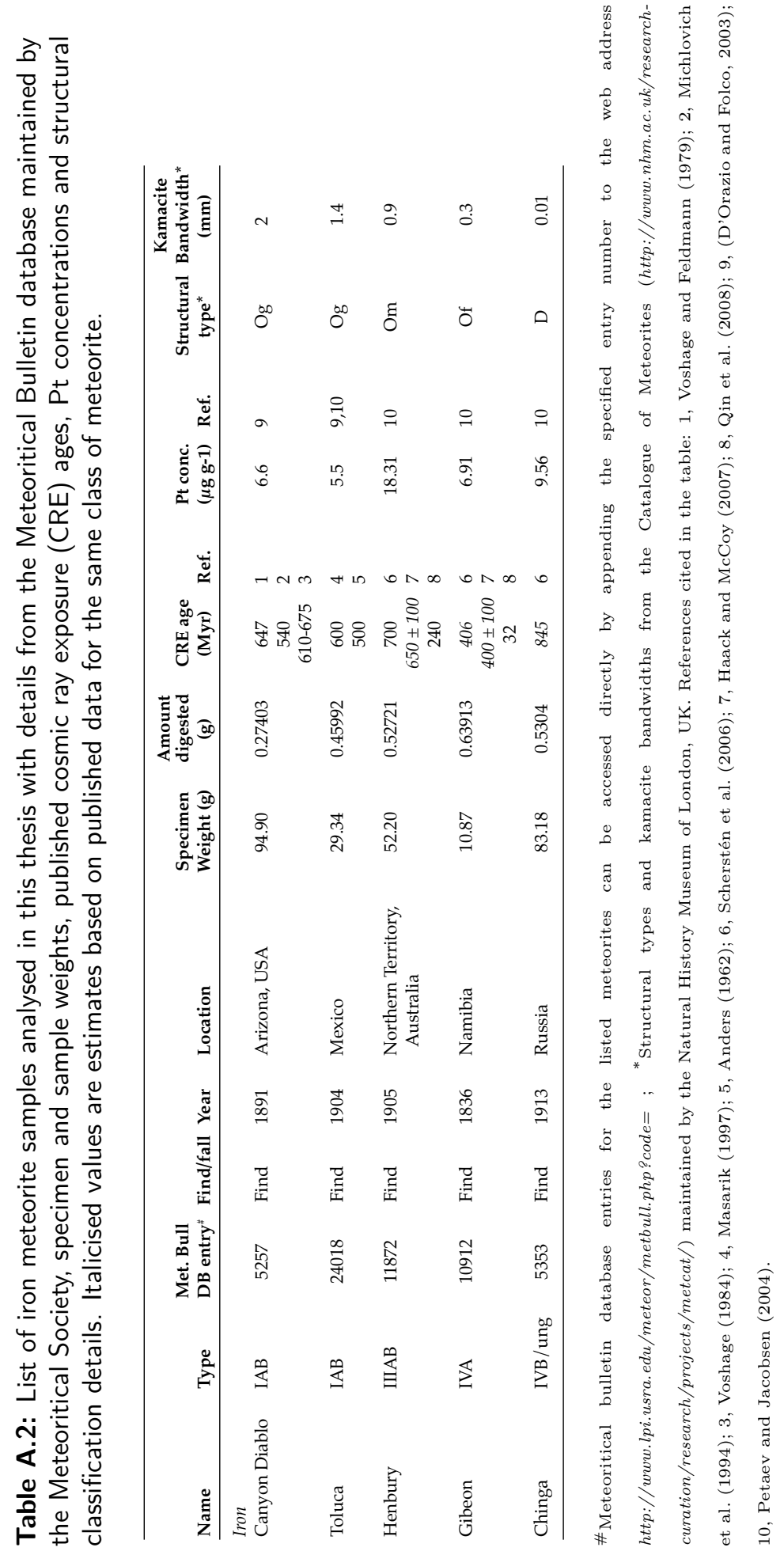




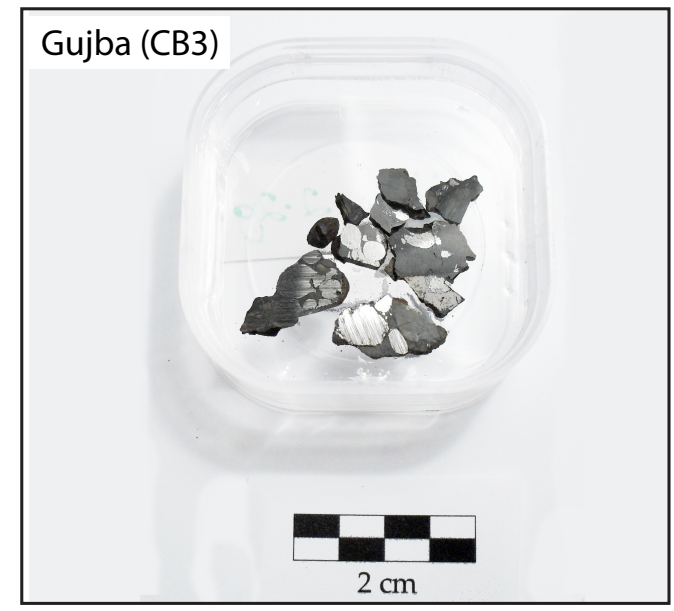

NWA 723 (CV3)
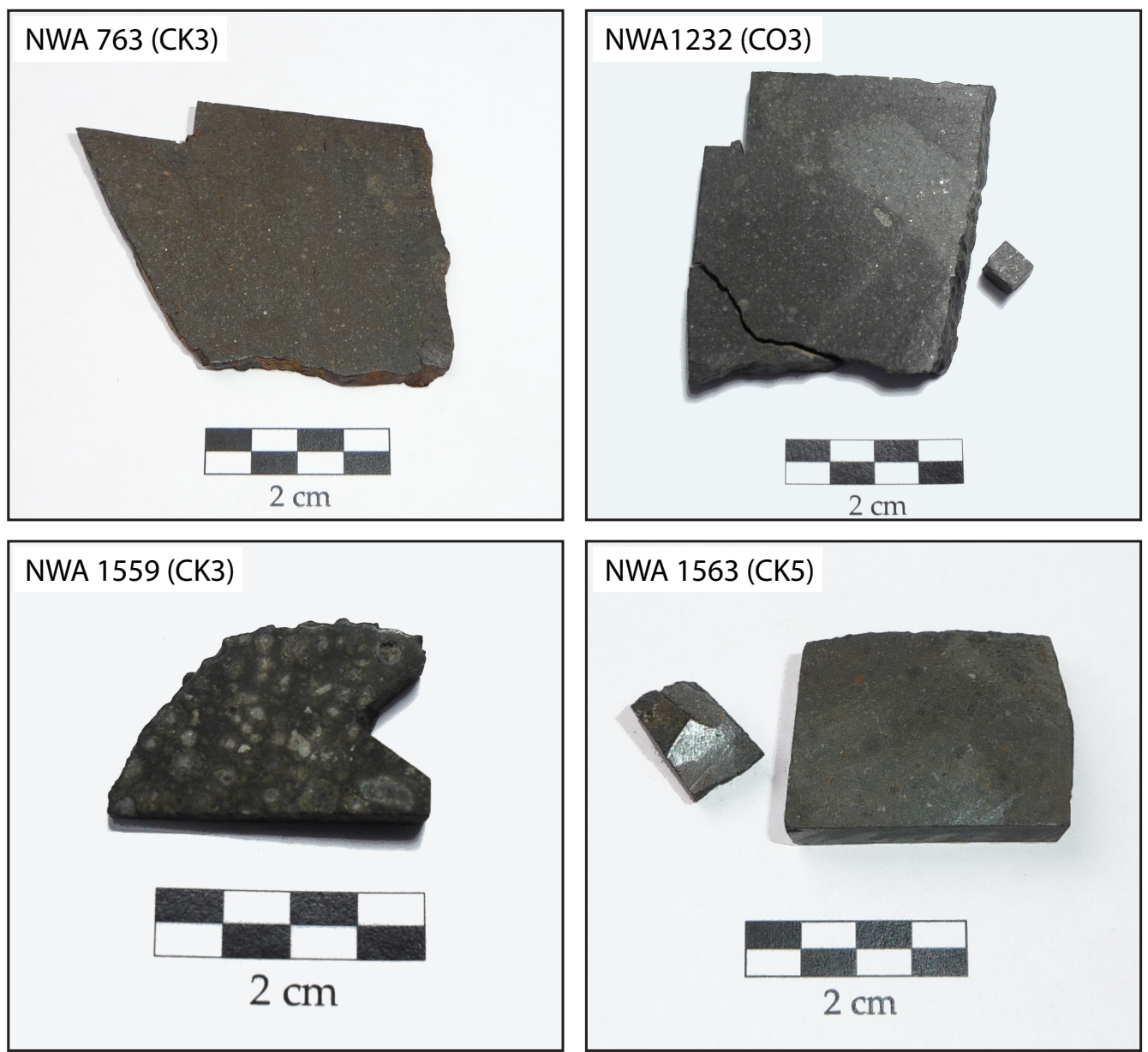

Figure A.1: Plain light photographs of meteorite samples analysed in this study (part 1). 

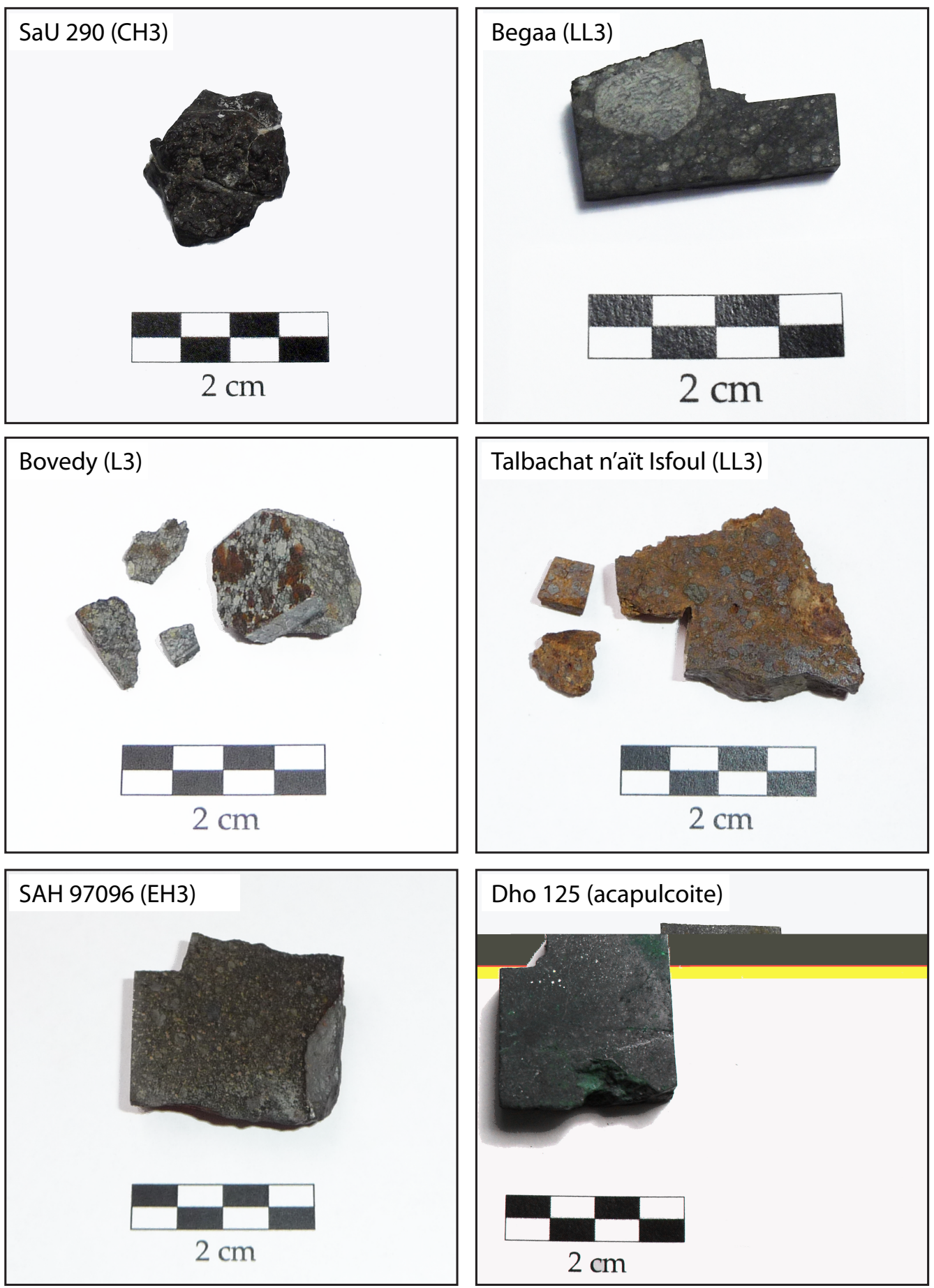

Figure A.1: Plain light photographs of meteorite samples analysed in this study (part 2). 

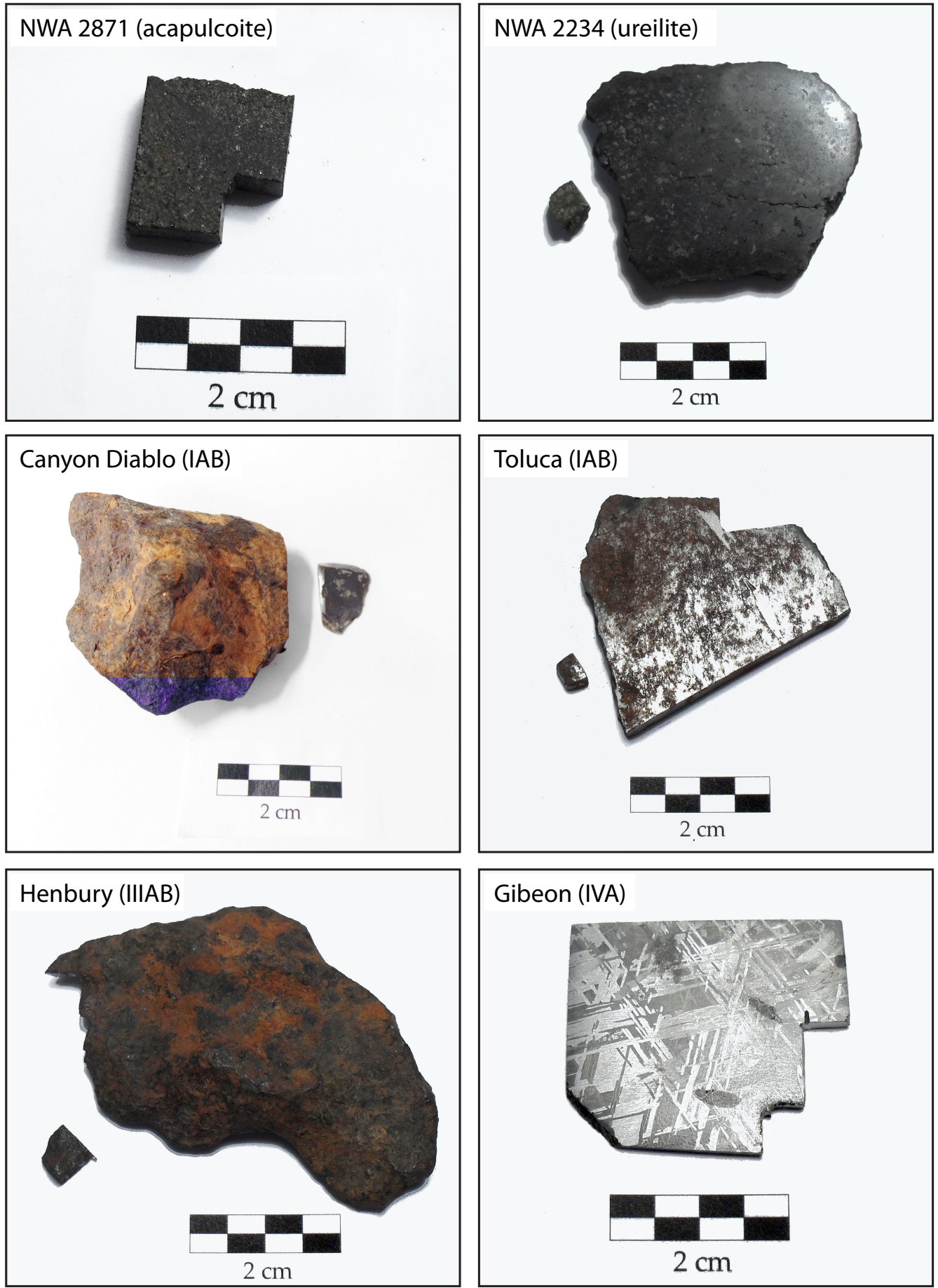

Figure A.1: Plain light photographs of meteorite samples analysed in this study (part 3). 


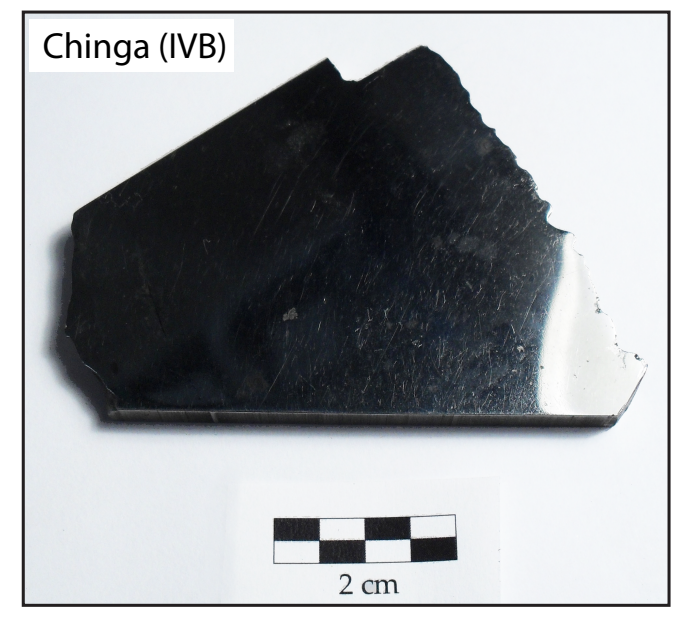

Figure A.1: Plain light photographs of meteorite samples analysed in this study (part 4). 


\section{Appendix B}

\section{Reprint of $J A A S$ paper}

Chapter 2 of this thesis has been published as an article in the Journal of Analytical Atomic Spectrometry. A reprint of the published article is included here, and can also be found online using the DOI: 10.1039/C3JA50022E. 


\section{Platinum stable isotope ratio measurements by double-spike multiple collector ICPMS†}

Cite this: J. Anal. At. Spectrom., 2013, 28, 853

Received 23rd January 2013

Accepted 4th March 2013

DOI: 10.1039/c3ja50022e

www.rsc.org/jaas

\author{
John Creech, ${ }^{\star a}$ Joel Baker, $\ddagger^{a}$ Monica Handler, ${ }^{a}$ Martin Schiller ${ }^{b}$ and Martin Bizzarro ${ }^{b}$
}

\section{Introduction}

A wide range of novel, non-traditional, stable isotope systems have recently been developed, largely as a result of the advent of multiple-collector inductively coupled plasma mass spectrometry (MC-ICPMS). These stable isotope systems are providing valuable new insights in the earth, environmental and planetary sciences. ${ }^{1-7}$ The platinum (Pt) stable isotope system represents a potentially powerful, but as yet unexplored, addition to this new suite of stable isotope systems. Platinum is a highly siderophile transition metal with six naturally occurring isotopes $-{ }^{190} \mathrm{Pt}$, ${ }^{192} \mathrm{Pt},{ }^{194} \mathrm{Pt},{ }^{195} \mathrm{Pt},{ }^{196} \mathrm{Pt}$ and ${ }^{198} \mathrm{Pt}$-with relative abundances of $\sim 0.01 \%, 0.78 \%, 32.86 \%, 33.78 \%, 25.21 \%$ and $7.36 \%$, respectively. ${ }^{8}$ Only ${ }^{190} \mathrm{Pt}$ is radioactive $\left(t_{1 / 2} \sim 10^{11}\right.$ years $)$, and

${ }^{a}$ School of Geography, Environment and Earth Sciences, Victoria University of Wellington, PO Box 600, Wellington, New Zealand.E-mail: john.creech@vuw.ac.nz ${ }^{b}$ Centre for Star and Planet Formation, Natural History Museum of Denmark, University of Copenhagen, DK-1350 Copenhagen, Denmark

$\dagger$ Electronic supplementary information (ESI) available. See DOI: 10.1039/c3ja50022e

$\ddagger$ Current address: School of Environment, The University of Auckland, Private Bag 92019, Auckland, New Zealand. despite its long half-life, is the basis of the ${ }^{190} \mathrm{Pt}-{ }^{186}$ Os decay system that has been used to infer interactions between Earth's core and mantle. ${ }^{9}$ Platinum can exist in a range of oxidation states, but principally occurs as $\mathrm{Pt}^{0}, \mathrm{Pt}^{2+}$ and $\mathrm{Pt}^{4+} \cdot{ }^{10}$ Unlike most previously studied non-traditional stable isotope systems, $\mathrm{Pt}$ is not utilised by any known essential biological mechanisms. However, the relatively large mass difference (2\%) between the abundant heavy and light isotopes of $\mathrm{Pt}$, coupled with its variable redox states in the Earth's core, mantle and surficial environment, means that it has potential to exhibit significant stable isotope variations in Earth materials. The bulk of Earth's Pt budget is thought to reside in the core as evidenced by the relative depletion of $\mathrm{Pt}$ and other highly siderophile elements in Earth's upper mantle relative to chondritic meteorites. ${ }^{11}$ Typical Pt concentrations in mantle rocks are $c a .1-100 \mathrm{ng} \mathrm{g}^{-1}$ as compared with 1-10 $\mu \mathrm{g} \mathrm{g}^{-1}$ in chondrites, iron meteorites and, presumably, Earth's core. As a result, the relative distributions of elements such as Pt between these different reservoirs are important in constraining models of planetary differentiation. ${ }^{11,12}$ Enrichments in Pt relative to the other platinum group elements (PGE; Rh, Ru, Pd, Os and Ir) have also been observed in authigenic marine sediments such as ferromanganese crusts 
and nodules. ${ }^{13-16}$ This Pt enrichment may be related to its variable oxidation states. ${ }^{13,17}$ As such, Pt stable isotopes hold considerable promise for use as an isotopic tracer for redox in surficial and marine environments and for processes relating to planetary accretion and differentiation.

In addition to being a potentially valuable geochemical tracer, Pt has a wide range of uses, including industrial, medical and scientific, where other potential applications for a Pt stable isotope tracer may be found. Various Pt compounds are used in medicine and, in particular, several different Pt complexes (cisplatin, carboplatin and oxaliplatin) are used in chemotherapy drugs. ${ }^{18-21}$ Platinum has applications in various manufacturing processes, usually as a catalyst, and is widely used in the automotive industry in catalytic converters. ${ }^{22,23}$ Given its use in automotive catalytic converters, $\mathrm{Pt}$ is also used for environmental monitoring of vehicle emissions by measuring Pt concentrations in roadside soils. ${ }^{24,25}$

Despite the potential range of uses for Pt stable isotopes, previous isotopic studies of Pt have been extremely limited. White et al. (1956) ${ }^{26}$ measured the isotopic composition of Pt using a double focusing mass spectrometer with a thermal ionisation source (Table 1). This Pt isotopic composition was used in IUPAC reports of elemental isotopic compositions and atomic weights until 1995, when a revised isotopic composition determined by Taylor et al. (1994) using gas source mass spectrometry and electron impact ionisation of gaseous $\mathrm{Pt}\left(\mathrm{PF}_{3}\right)_{4}$ was adopted $^{27,28}$ (Table 1). In the late 1990s, two studies documented procedures for measurement of Pt isotopes by negative thermal ionisation mass spectrometry (N-TIMS). ${ }^{29,30}$ One of the groups subsequently developed a Pt isotope reference material (IRMM010) for the Institute for Reference Materials and Measurements, for which certified values were determined using quadrupole
ICPMS $^{31}$ (Table 1). Later studies of IRMM-010 have been limited to its use in testing the analytical performance of axial inductively coupled plasma time-of-flight mass spectrometer instruments. $^{32,33}$ Two recently published studies used internally normalised Pt isotope ratios as a dosimeter for neutron capture in iron meteorites. ${ }^{34,35}$ However, to-date there has been no investigation of the Pt stable isotopic system.

Herein, we describe a method for highly precise determination of $\mathrm{Pt}$ stable isotope ratios using ${ }^{192} \mathrm{Pt}-{ }^{198} \mathrm{Pt}$ and ${ }^{196} \mathrm{Pt}-{ }^{198} \mathrm{Pt}$ double-spikes (DS) to correct for instrumental mass bias. We show that this approach is capable of measuring Pt stable isotopes on $c a$. 40-85 ng of natural Pt with an external reproducibility of $\leq \pm 10 \mathrm{ppm} \mathrm{u}^{-1}$ ( $2 \mathrm{sd}$; $u$ is the unified atomic mass unit), and identify measurable $\mathrm{Pt}$ stable isotope variations amongst commercially available $\mathrm{Pt}$ standards. Moreover, during the course of this work, we identified that the previously adopted abundances of some $\mathrm{Pt}$ isotopes are in relative terms up to $10 \%$ inaccurate and, as such, we present a revised schedule of the isotopic abundances and atomic weight of Pt.

\section{Double-spike methodology}

The DS technique has been extensively used in highly precise $\mathrm{Pb}$ isotope analysis, and has also been applied to a wide range of other isotope systems, including $\mathrm{S}, \mathrm{Ca}, \mathrm{Cr}, \mathrm{Zn}, \mathrm{Fe}, \mathrm{Mo}, \mathrm{Cd}, \mathrm{Ba}$ and Os, by both MC-ICPMS and TIMS analysis. ${ }^{36-49}$ The fundamentals of the DS approach for isotopic analysis have been well documented, ${ }^{50-56}$ and mathematical solutions have been developed utilising both algebraic ${ }^{56,57}$ and geometric $\mathrm{c}^{45,49,54,55,58}$ approaches. Here we use the algebraic method of Rudge et al. (2009). ${ }^{56}$

Table 1 Isotopic composition (in \%) of natural platinum, Oak Ridge isotopic spikes and prepared double-spikes ${ }^{a}$

\begin{tabular}{|c|c|c|c|c|c|c|c|c|}
\hline \multicolumn{2}{|c|}{ Material [ref.] } & ${ }^{190} \mathrm{Pt}$ & ${ }^{192} \mathrm{Pt}$ & \multicolumn{2}{|l|}{${ }^{194} \mathrm{Pt}$} & ${ }^{195} \mathrm{Pt}$ & ${ }^{196} \mathrm{Pt}$ & ${ }^{198} \mathrm{Pt}$ \\
\hline \multicolumn{2}{|c|}{ Natural Pt [8] } & 0.012 & 0.782 & \multicolumn{2}{|c|}{32.864} & 33.776 & 25.210 & 7.356 \\
\hline \multicolumn{2}{|c|}{ Oak Ridge ${ }^{192}$ Pt spike [56] } & 0.01 & 56.93 & \multicolumn{2}{|c|}{26.34} & 11.12 & 4.77 & 0.83 \\
\hline \multicolumn{2}{|c|}{ Oak Ridge ${ }^{196}$ Pt spike [56] } & 0.98 & 0.98 & \multicolumn{2}{|l|}{0.62} & 1.54 & 95.6 & 0.28 \\
\hline \multicolumn{2}{|c|}{ Oak Ridge ${ }^{198}$ Pt spike [56] } & 0.05 & 0.05 & \multicolumn{2}{|l|}{0.83} & 1.23 & 2.23 & 95.61 \\
\hline \multicolumn{2}{|c|}{ Optimal ${ }^{192} \mathrm{Pt}-{ }^{198} \mathrm{Pt}$ DS [56] } & 0.03 & 22.18 & \multicolumn{2}{|l|}{10.75} & 5.08 & 3.22 & 58.74 \\
\hline & 0.31 & 0.31 & \multicolumn{2}{|l|}{0.77} & 1.31 & 28.1 & 69.2 \\
\hline \multicolumn{2}{|c|}{${ }_{196}^{192}$ Pt spike [this study] } & 0.0056 & 57.17 & \multicolumn{2}{|l|}{26.13} & 11.08 & 4.75 & 0.87 \\
\hline \multirow{2}{*}{\multicolumn{2}{|c|}{${ }^{196} \mathrm{Pt}$ spike [this study] }} & 0.0002 & 0.01 & \multicolumn{2}{|l|}{1.47} & 3.58 & 94.47 & 0.47 \\
\hline & his study] & 0.0003 & 0.03 & \multicolumn{2}{|l|}{1.74} & 2.80 & 3.70 & 91.74 \\
\hline \multicolumn{2}{|c|}{${ }^{192} \mathrm{Pt}-{ }^{198} \mathrm{Pt}$ DS [this study] } & 0.0028 & 26.21 & \multicolumn{2}{|l|}{12.88} & 6.58 & 4.17 & 50.16 \\
\hline \multicolumn{2}{|c|}{${ }^{196} \mathrm{Pt}-{ }^{198} \mathrm{Pt}$ DS [this study] } & 0.0003 & 0.03 & \multicolumn{2}{|l|}{1.68} & 2.97 & 23.47 & 71.85 \\
\hline Year & Method [ref.] & ${ }^{190} \mathrm{Pt}$ & ${ }^{192} \mathrm{Pt}$ & ${ }^{194} \mathrm{Pt}$ & ${ }^{195} \mathrm{Pt}$ & ${ }^{196} \mathrm{Pt}$ & ${ }^{198} \mathrm{Pt}$ & Atomic wt \\
\hline 1947 & Unpublished $^{b}[26]$ & 0.09 & 0.78 & 32.8 & 33.7 & 25.4 & 7.23 & $195.079^{c}$ \\
\hline 1956 & Single collector TIMS [26] & $0.0127(5)$ & $0.78(10)$ & $32.9(1)$ & $33.8(1)$ & $25.2(1)$ & $7.19(4)$ & $195.079^{c}$ \\
\hline 1984 & Compiled [59] & $0.01(1)$ & $0.79(50)$ & $32.9(5)$ & $33.8(1)$ & $25.3(5)$ & $7.2(2)$ & 195.080 \\
\hline 1996 & Gas Source MS [28] & $0.013634(68)$ & $0.782659(35)$ & $32.967(77)$ & $33.831557(42)$ & $25.24166(36)$ & $7.16349(42)$ & $195.078^{d}$ \\
\hline 2002 & MC-ICPMS [65] & $0.01292(2)$ & 0.80168 & 32.93367 & 33.79892 & 25.21481 & 7.240 & \\
\hline 2002 & Quadrupole ICPMS [31] & $0.01172(58)$ & $0.7819(80)$ & $32.864(140)$ & $33.776(79)$ & $25.210(11)$ & $7.356(43)$ & $195.0844(58)$ \\
\hline This study ${ }^{e}$ & MC-ICPMS [this study] & $0.01289(6)$ & $0.79376(42)$ & 32.8078 & $33.7871(40)$ & $25.290(10)$ & $7.308(11)$ & $195.08395(68)$ \\
\hline
\end{tabular}

${ }^{a}$ Uncertainties on last digits are given in parentheses. ${ }^{b}$ Inghram et al. (1947) unpublished data given in White et al. ${ }^{26}{ }^{c}$ Atomic weight from De Bievre et al. ${ }^{59} d$ Atomic weight from Rosman and Taylor. ${ }^{60} e^{2}$ Mean of NZ and DK isotope ratios with uncertainties based on variance between NZ and DK measurements. 
The DS method is commonly accepted as the most rigorous method available for correcting for instrumental mass fractionation as it has several advantages over other methods such as standard-sample bracketing, which are: (1) for stable isotope systems where mass-dependent fractionation is being studied, DS-corrected isotope ratios can be obtained from a single analysis; $^{56}$ (2) the DS can potentially correct for any massdependent effects to the sample after addition of the DS, such as isotopic fractionations that might occur as a result of incomplete yields during chemical separation of the element from natural samples; ${ }^{56,57}$ (3) the DS method has been shown to be less sensitive to the presence of minor amounts of remaining matrix elements than sample-standard bracketing methods. ${ }^{41}$

\subsection{Pt double-spike design}

With five stable isotopes and one with an extremely long halflife $\left({ }^{190} \mathrm{Pt}, t_{1 / 2} \sim 10^{11}\right.$ years $), \mathrm{Pt}$ is ideally suited to the DS approach. Error propagation in DS calculations needs to be minimised by selection of an optimal spike composition and sample-spike mixtures. A database of theoretically ideal spike compositions and spike/sample ratios for all elements with four or more naturally occurring isotopes was presented by Rudge et al., ${ }^{56}$ which includes optimal DS compositions based on available spikes from the Oak Ridge National Laboratory, USA. Two platinum DS were prepared as part of this study, as described below.

2.1.1 ${ }^{192} \mathbf{P t}-{ }^{198} \mathrm{Pt}$ double-spike. The ${ }^{192} \mathrm{Pt}-{ }^{198} \mathrm{Pt}$ DS comprises a mixture of $38.9 \%{ }^{192} \mathrm{Pt}$ and $61.1 \%{ }^{198} \mathrm{Pt}$ spikes. This DS combination has one of the smallest error propagations predicted by the double-spike toolbox of Rudge et al. ${ }^{56}$ (ca. 32 ppm u $\left.{ }^{-1}, 1 \mathrm{sd}\right)$. The ${ }^{192} \mathrm{Pt}-{ }^{198} \mathrm{Pt}$ DS inversion was performed using ${ }^{192} \mathrm{Pt},{ }^{194} \mathrm{Pt},{ }^{196} \mathrm{Pt}$ and ${ }^{198} \mathrm{Pt}$. Given that the spiked isotopes ${ }^{192} \mathrm{Pt}$ and ${ }^{198} \mathrm{Pt}$ have relatively low natural abundances $(0.78 \%$ and $7.36 \%$, respectively; ${ }^{8}$ ) and the two unspiked isotopes in the inversion $\left({ }^{194} \mathrm{Pt}\right.$ and $\left.{ }^{196} \mathrm{Pt}\right)$ have high natural abundances, this DS permits precise isotopic determinations over a wide range of sample/spike ratios. The use of this DS is, however, complicated by a potentially large direct isobaric interference from Os on ${ }^{192} \mathrm{Pt}$ and the fact that, in some natural materials that might be targets for Pt stable isotope analysis, Pt and Os are both of similar abundance (e.g. chondritic and iron meteorites, Earth's mantle). ${ }^{190} \mathrm{Pt}$ and ${ }^{192} \mathrm{Pt}$ isotopes have isobars with abundant isotopes of osmium $\left({ }^{190} \mathrm{Os}\right.$ and ${ }^{192} \mathrm{Os}$ with relative abundances of $26.2 \%$ and $40.8 \%$, respectively). Thus, the presence of even small amounts of Os in a sample can significantly affect the Pt signal on those masses, producing inaccurate ${ }^{192} \mathrm{Pt}-{ }^{198} \mathrm{Pt}$ DS corrected ratios.

2.1.2 ${ }^{196} \mathbf{P t}-{ }^{198} \mathbf{P t}$ double-spike. The ${ }^{196} \mathrm{Pt}-{ }^{198} \mathrm{Pt}$ DS comprises a mixture of $27.7 \%{ }^{196} \mathrm{Pt}$ and $72.3 \%{ }^{198} \mathrm{Pt}$ spikes. The hypothetical error propagation on the ${ }^{196} \mathrm{Pt}-{ }^{198} \mathrm{Pt}$ DS is slightly larger than that of the ${ }^{192} \mathrm{Pt}-{ }^{198} \mathrm{Pt}$ DS ( $c a .40 \mathrm{ppm} \mathrm{u}^{-1} ; 1 \mathrm{sd}$ ). However, as alluded to above, the ${ }^{196} \mathrm{Pt}-{ }^{198} \mathrm{Pt}$ DS has some potential advantages over the ${ }^{192} \mathrm{Pt}-{ }^{198} \mathrm{Pt}$ DS in that the ${ }^{196} \mathrm{Pt}-{ }^{198} \mathrm{Pt}$ double-spike inversion is performed on masses ${ }^{194} \mathrm{PtPt},{ }^{195} \mathrm{Pt}$, ${ }^{196} \mathrm{Pt}$ and ${ }^{198} \mathrm{Pt}$, and this DS should thus be unaffected by the presence of Os.

\section{Materials and methods}

\subsection{Chemicals and standard solutions}

Optima ${ }^{\mathrm{TM}}$ grade nitric $\left(\mathrm{HNO}_{3}\right)$ and hydrochloric $(\mathrm{HCl})$ acids were used as supplied without further purification. Acid dilutions were performed with ultrapure $(>18 \mathrm{M} \Omega \mathrm{cm})$ water. All sample handling and preparation was performed in a class-100 ultra-clean laboratory at Victoria University of Wellington.

The Pt isotope standard IRMM-010 was purchased from the Institute for Reference Materials and Measurements, Belgium. ${ }^{31}$ The standard was supplied in the form of Pt wire segments, which were cleaned prior to digestion by sequentially rinsing in ultrapure water, analytical grade methanol and dilute $(5 \%)$ $\mathrm{HNO}_{3}$. The IRMM-010 material was carefully weighed, and then digested in aqua regia $\left(\mathrm{HCl}: \mathrm{HNO}_{3}\right.$ prepared in a volume ratio of $3: 1$ ) in a Savillex ${ }^{\mathrm{TM}}$ teflon beaker. The IRMM-010 Pt solution was dried down, refluxed with concentrated $\mathrm{HNO}_{3}$, evaporated to dryness, and finally redissolved in $7 \mathrm{M} \mathrm{HNO}_{3}$. At the time this standard was prepared, sample introduction was carried out in $\mathrm{HNO}_{3}$. This was later changed to $\mathrm{HCl}$, therefore a portion of this standard was converted to chloride form and stored in $20 \% \mathrm{HCl}$.

Two commercially available Pt ICPMS standards were used in this study: J. T. Baker $1000 \mu \mathrm{g} \mathrm{mL}^{-1} \mathrm{Pt}$ ICP std in $20 \% \mathrm{HCl}$ (Lot\# H44N77), and SPEX CertiPrep $1000 \mu \mathrm{g} \mathrm{mL} \mathrm{m}^{-1} \mathrm{Pt}$ ICP standard in 10\% $\mathrm{HCl}$ from Fisher Scientific (Lot\# 15-181PT; hereafter referred to as the Baker and Spex standards, respectively). Additionally, a piece of Pt metal ribbon used for TIMS filaments was obtained and digested for use as an in-house standard (hereafter referred to as the Ribbon standard). The Pt Ribbon standard was pre-cleaned and digested in the same manner as described above for IRMM-010. The digested filament was evaporated to dryness several times in concentrated $\mathrm{HCl}$, and finally redissolved in $20 \% \mathrm{HCl}$.

\subsection{Preparation of platinum double-spikes}

Three single Pt isotope spikes $\left({ }^{192} \mathrm{Pt},{ }^{196} \mathrm{Pt}\right.$ and $\left.{ }^{198} \mathrm{Pt}\right)$ were obtained from the Oak Ridge National Laboratory, USA (Batch numbers 186140, 235601 and 235701, respectively). Reference Pt isotopic compositions for each isotopic spike are shown in Table 1 as well as the measured compositions obtained in this study. The ${ }^{192} \mathrm{Pt}$ spike was supplied as a segment of Pt metal ribbon, and the ${ }^{196} \mathrm{Pt}$ and ${ }^{198} \mathrm{Pt}$ spikes were provided as very finegrained metallic powders. The ${ }^{192} \mathrm{Pt}$ spike ribbon was sequentially cleaned prior to digestion by rinsing with ultrapure water, analytical grade methanol and dilute $(5 \%) \mathrm{HNO}_{3}$. Each spike was digested independently with aqua regia in Savillex ${ }^{\mathrm{TM}}$ teflon beakers. Once the spike material had been digested, the spike solutions were repeatedly evaporated to dryness in concentrated $\mathrm{HCl}$, and finally redissolved in $20 \% \mathrm{HCl}$.

Aliquots of each single spike solution were taken to determine their concentrations (and isotopic compositions). These were measured by MC-ICPMS using a standard bracketing approach with the IRMM-010 standard. The two DS were then prepared by mixing the appropriate two spikes in the proportions calculated for optimal ${ }^{192} \mathrm{Pt}-{ }^{198} \mathrm{Pt}$ and ${ }^{196} \mathrm{Pt}-{ }^{198} \mathrm{Pt}$ DS mixtures as given by Rudge et al. ${ }^{56}$ Each DS mixture was then 
evaporated to dryness, taken up in a small volume of concentrated $\mathrm{HCl}$, evaporated to dryness again, and finally taken up in $6 \mathrm{M} \mathrm{HCl}$. Accurate isotopic determination by the DS technique requires calibration of the DS composition. The method for calibration of the DS prepared here and the accuracy of the calibrations are described below.

Platinum standards were spiked immediately prior to MCICPMS measurement in proportions close to that for optimal spiking as given in Rudge et al. ${ }^{56}$ Reference Pt isotopic compositions for each isotopic spike and DS are shown in Table 1 along with the compositions measured by MC-ICPMS for the spikes and DS used in this study.

\subsection{Mass spectrometry}

Platinum isotope measurements were performed using MCICPMS instruments in two different laboratories. Most analyses were carried out with a Nu Plasma HR MC-ICPMS (Nu Instruments) at Victoria University of Wellington, New Zealand. Repeated measurements of selected standards were also undertaken with a ThermoFisher Neptune MC-ICPMS located at the Centre for Star and Planet Formation, Natural History Museum of Denmark, University of Copenhagen, Denmark. Data from these two laboratories are annotated as being from NZ or DK in the following text, tables and figures. Measurement conditions were kept as similar as possible between the instruments. Typical operating conditions and measurement parameters are summarised in Table 2, and collector configurations for both instruments are given in Table S1. $\dagger$ Both instruments were operated in low-resolution mode. Samples were introduced to both instruments in $0.5 \mathrm{M} \mathrm{HCl}$, as $\mathrm{HCl}$ was found to result in better washouts over $\mathrm{HNO}_{3}$.

3.3.1 Nu Plasma MC-ICPMS (NZ). The Nu Plasma MCICPMS was operated in low-resolution mode, with a mass resolution of $\sim 1840(M / \Delta M$; peak edge width from $5-95 \%$ full peak height). A Nu Instruments DSN-100 desolvation nebuliser system with an ESI PFA micro-flow nebuliser was used for Pt solution introduction to the mass spectrometer. Measurements were typically made on $0.5 \mathrm{M} \mathrm{HCl}$ solutions with 75 or $60 \mathrm{ng}$ $\mathrm{mL}^{-1}$ (for the ${ }^{192} \mathrm{Pt}-{ }^{198} \mathrm{Pt}$ or ${ }^{196} \mathrm{Pt}-{ }^{198} \mathrm{Pt} \mathrm{DS}$, respectively) of natural Pt, with an almost equivalent contribution from DS Pt. The sample uptake rate was $c a .80 \mu \mathrm{L} \mathrm{min}^{-1}$, and the sensitivity of the instrument was typically $c a .280 \mathrm{~V} \mathrm{ppm}^{-1}$ total Pt under these conditions. Peak centering was performed at the start of each session and checked at the end of the session. Each individual analysis comprised $100 \times 8 \mathrm{~s}$ integrations, corresponding to $13.3 \mathrm{~min}$ of data acquisition, and consumed $c a .80 \mathrm{ng}$ of natural Pt. Baselines were obtained by measuring on-peak for $100 \times 8 \mathrm{~s}$ integrations prior to each analysis. A washout time of ca. $30 \mathrm{~min}$ was applied between analyses and baseline measurements. Using this approach, background levels were typically $\ll 1 \mathrm{mV}$ on all Pt isotopes.

3.3.2 Thermo Neptune plus MC-ICPMS (DK). The Neptune MC-ICPMS was operated in low-resolution mode, with a mass resolution of $\sim 1600(M / \Delta M$ as defined by the peak edge width from 5-95\% full peak height). An ESI Apex IR desolvating nebuliser sample introduction system was used without nitrogen input and with an Actively Cooled Membrane (ACM) add-on module for additional membrane desolvation. The sample uptake rate was $c a$. $30 \mu \mathrm{L} \mathrm{min}{ }^{-1}$, and the sensitivity of the instrument under these conditions was typically $\sim 100 \mathrm{~V} \mathrm{ppm}^{-1}$ total Pt. Solutions were prepared with a natural Pt concentration of $100 \mathrm{ng} \mathrm{mL}{ }^{-1}$ and an almost equivalent additional Pt contribution from DS. Peak centering was performed at the start of each analysis, and each analysis comprised $100 \times 8.4$ s integrations. Each analysis corresponded to $14 \mathrm{~min}$ of data acquisition and consumed $c a .40 \mathrm{ng}$ of natural Pt. Baselines were obtained by on-peak measurements for $100 \times 8.4 \mathrm{~s}$ integrations

Table 2 Instrumental operating and measurement conditions

\begin{tabular}{|c|c|c|}
\hline & Nu Plasma (NZ) & Neptune (DK) \\
\hline \multicolumn{3}{|l|}{ Instrument operating conditions } \\
\hline RF power & $1300 \mathrm{~W}$ & $1200 \mathrm{~W}$ \\
\hline Plasma gas flow rate & $14 \mathrm{~L} \mathrm{~min}^{-1}$ & $15 \mathrm{~L} \mathrm{~min}^{-1}$ \\
\hline Interface cones $\quad$ Sampler & Nickel & Jet cone \\
\hline Skimmer & High-sensitivity nickel & $\mathrm{X}$-cone \\
\hline Source slit width & $0.3 \mathrm{~mm}$ & $0.25 \mathrm{~mm}$ \\
\hline Acceleration voltage & $6 \mathrm{kV}$ & $10 \mathrm{kV}$ \\
\hline Instrument resolution & $\sim 1840$ & $\sim 1600$ \\
\hline Mass analyser pressure & $5-10 \times 10^{-9} \mathrm{mbar}$ & ca. $8 \times 10^{-9} \mathrm{mbar}$ \\
\hline Detector & $\begin{array}{l}12 \text { Faraday detectors; all Pt } \\
\text { channels using } 10^{11} \Omega \text { resistors }\end{array}$ & $\begin{array}{l}9 \text { Faraday detectors; all Pt } \\
\text { channels using } 10^{11} \Omega \text { resistors }\end{array}$ \\
\hline Sample introduction system & $\mathrm{Nu}$ Instruments DSN-100 & ESI Apex IR w/ACM \\
\hline Sample uptake rate & $\sim 80 \mu \mathrm{L} \mathrm{min} \operatorname{mon}^{-1}$ & $\sim 30 \mu \mathrm{L} \mathrm{min} \min ^{-1}$ \\
\hline \multicolumn{3}{|l|}{ Measurement parameters } \\
\hline Solution concentration & $60-75 \mathrm{ppb}$ & $100 \mathrm{ppb}$ \\
\hline Typical sensitivity & $\sim 280 \mathrm{~V} \mathrm{ppm}^{-1}$ total $\mathrm{Pt}^{a}$ & $\sim 100 \mathrm{~V} \mathrm{ppm}^{-1}$ total $\mathrm{Pt}^{a}$ \\
\hline Sample measurement time & $100 \times 8 \mathrm{~s}$ integrations & $100 \times 8.4 \mathrm{~s}$ integrations \\
\hline Washout time & $30 \mathrm{~min}$ & $30 \mathrm{~min}$ \\
\hline Background measurement time & $100 \times 8 \mathrm{~s}$ integrations & $100 \times 8.4 \mathrm{~s}$ integrations \\
\hline
\end{tabular}

${ }^{a}$ At specified uptake rate and concentration. 
prior to and following each analysis. A washout time of $30 \mathrm{~min}$ was used between sample analyses and baseline measurements. Using this approach, background levels were typically $<1 \mathrm{mV}$ for all Pt isotopes.

\subsection{Interference correction}

Isobaric interferences on Pt exist from Os (masses 190 and 192) and $\mathrm{Hg}$ (masses 196 and 198). These overlapping masses, as well as most relevant molecular species, have mass differences that are too small to be resolved at the mass resolving capability of the MC-ICPMS instruments used in this study (Table S1†). Contributions from $\mathrm{Os}$ and $\mathrm{Hg}$ were monitored using interference-free masses ${ }^{188} \mathrm{Os}$ and ${ }^{200} \mathrm{Hg}$. Due to its extremely volatile nature, interferences from $\mathrm{Hg}$ in natural samples are unlikely to be significant. However, due to the high relative abundances of ${ }^{190} \mathrm{Os}$ and ${ }^{192}$ Os (26.4\% and $41 \%$, respectively), Pt isotope analyses at these masses are likely to be severely impacted by the presence of even small amounts of Os. In all of the undoped analyses presented here, signals on monitored $\mathrm{Os}$ and $\mathrm{Hg}$ masses were negligible (i.e. $<15 \mu \mathrm{V})$. An interference correction procedure for Os based on ref. 61 was tested. The interference-free ${ }^{189} \mathrm{Os} /{ }^{188} \mathrm{Os}$ ratio (1.21967 (ref. 62)) was used to calculate the Os mass bias, which was then used to calculate the Os interference corrections at masses 190 and 192 from the ${ }^{188}$ Os signal intensity.

In all of the undoped analyses presented here, signals on monitored $\mathrm{Os}$ and $\mathrm{Hg}$ masses were indistinguishable from the baseline noise.

\subsection{Data reduction}

All data reduction was conducted off-line using Iolite, a freely available data reduction package that runs within the Igor Pro software from Wavemetrics. ${ }^{63}$ An add-on has been developed for processing DS data within Iolite (Creech and Paul, in prep.), which can be adapted for any DS system. This procedure will be made freely available for download from the Iolite website (http://www.iolite.org.au). The DS calculations are performed algebraically using the equations of Rudge et al. ${ }^{56}$

\subsection{Reporting of platinum stable isotope data}

In non-traditional stable isotope systems, particularly in the intermediate- to heavy-mass range, it is conventional to report isotopic compositions as a ratio of a heavy over a light isotope for a given element $\left(\right.$ e.g. $\left.{ }^{44} \mathrm{Ca} /{ }^{40} \mathrm{Ca}\right)$, as is the case for traditional stable isotope systems. ${ }^{64}$ We report results here using that approach. Given that the abundances of the two lightest Pt isotopes $\left({ }^{190} \mathrm{Pt}\right.$ and $\left.{ }^{192} \mathrm{Pt}\right)$ are very small, we use ${ }^{194} \mathrm{Pt}$ as the denominator isotope. Typically, in stable isotope studies, the highest mass isotope of a reasonably high abundance would be chosen for the numerator isotope. Therefore, we report Pt stable isotopes in terms of ${ }^{198} \mathrm{Pt} /{ }^{194} \mathrm{Pt}$ ratios. Given that mass-dependent natural stable isotope fractionations in Pt isotopes might be expected to be relatively small (i.e. parts-per-million), we have adopted a $\mu$ notation representing the parts per million deviation of the ${ }^{198} \mathrm{Pt} /{ }^{194} \mathrm{Pt}$ ratio from the same ratio for the IRMM$010 \mathrm{Pt}$ isotope standard. Isotopic compositions are thus reported as $\mu^{198} \mathrm{Pt}$ :

$$
\mu^{198} \mathrm{Pt}=\left[\frac{\frac{{ }^{198} \mathrm{Pt}}{{ }^{194} \mathrm{Pt}_{\text {sample }}}}{\frac{{ }^{198} \mathrm{Pt}}{{ }^{194} \mathrm{Pt}_{\text {IRMM-010 }}}}-1\right] \times 10^{6}
$$

Assuming fractionation occurs through a mass-dependent process, stable isotope fractionations may also be expressed in terms of the relative difference per unified atomic mass unit (i.e. $\mathrm{ppm} \mathrm{u}^{-1}$ ).

\subsection{Analytical tests}

We conducted the following tests to demonstrate the veracity of our technique: (1) mixtures of the IRMM-010 and both DS were measured over a range of different mixing proportions to test the accuracy of the DS calibrations; (2) replicate DS measurements of the IRMM-010 standard were made over many months to assess both the within-session and long-term reproducibility of the technique; (3) a range of element doping tests were conducted to test the sensitivity of the technique to the presence of contaminants (particularly Os) that may be present, for example, following chemical separation of Pt from natural samples; (4) the potential effects arising from imperfect peak positioning or lower ion beam intensities were examined by analysis off the peak centre and in low Pt concentration solutions; (5) Pt stable isotope compositions of three different Pt standards were measured to investigate whether any variability in Pt stable isotope compositions exists.

\section{Results and discussion}

\subsection{Platinum isotopic composition of the IRMM-010 standard}

The currently accepted reference Pt isotope composition for IRMM-010 was determined using partially calibrated analyses by quadrupole ICPMS in 2002. ${ }^{31}$ However, Pt isotope ratios reported by Morgan et al. ${ }^{65}$ measured by MC-ICPMS show significant differences in isotopic composition for some $\mathrm{Pt}$ isotopes as compared with those of ref. 31. During this study, it was found that no DS calibration would yield consistent DS-corrected isotope ratios using the natural Pt isotopic composition reported by ref. 31, suggesting that the reference values for IRMM-010 may be inaccurate. Therefore, we elected to remeasure the isotopic composition of IRMM-010 by MC-ICPMS. Platinum isotope measurements of IRMM-010 were carried out using external normalisation to the $\mathrm{Pb}$ isotope standard SRM981. Lead was selected for performing the mass bias correction as it is the closest element in mass to Pt that does not have any overlapping isobaric interferences on $\mathrm{Pt}$, and $\mathrm{Pb}$ isotopic reference standards with well constrained isotope ratios are available. Solutions of IRMM-010 were doped with the Pb isotope standard SRM981 at ca. $10 \%$ of the Pt concentration and an internal mass bias correction was performed using a ${ }^{208} \mathrm{~Pb} /{ }^{206} \mathrm{~Pb}$ ratio of $2.16775 .{ }^{38}$ This ${ }^{208} \mathrm{~Pb} /{ }^{206} \mathrm{~Pb}$ ratio for SRM981 was determined using the $\mathrm{Pb}$ DS approach referenced to $\mathrm{a}^{208} \mathrm{~Pb} /{ }^{206} \mathrm{~Pb}$ ratio of 1.00016 for the $\mathrm{Pb}$ isotope standard SRM982. The mass-bias-corrected ${ }^{207} \mathrm{~Pb} /{ }^{206} \mathrm{~Pb}$ ratio $\left({ }^{207} \mathrm{~Pb} /{ }^{206} \mathrm{~Pb} \mathrm{FC}\right)$ was also measured and 
compared with the reference value of $0.91489 .{ }^{38}$ The following factors must be taken into account when considering the accuracy of this approach to determining Pt isotope ratios:

(1) The potential presence of Pt oxide and nitride interferences on $\mathrm{Pb}$ isotopes (Table $\mathrm{S} 1 \dagger$ ), which must either be insignificant or corrected for. To account for these potential interferences, measurements of Pb-doped Pt standards were bracketed with analyses of an undoped Pt standard solution with an identical Pt concentration, and the signals observed in the undoped $\mathrm{Pt}$ standard at the $\mathrm{Pb}$ masses were subtracted from $\mathrm{Pb}$ doped analyses. The contributions on $\mathrm{Pb}$ masses from interferences were $c a .1 \mathrm{mV}$ for undoped $150 \mathrm{ppb}$ IRMM-010 solutions, compared with signals of $2-5 \mathrm{~V}$ in $\mathrm{Pb}$ doped analyses.

(2) That the relative mass bias behaviour of $\mathrm{Pb}$ and $\mathrm{Pt}$ are similar. Although the mass bias behaviour is unlikely to be identical for $\mathrm{Pt}$ and $\mathrm{Pb}$, this assumption is still sufficient to explore and further refine the Pt isotopic composition of IRMM010 given that the studies of ref. 31 and 65 yield differences of $1-10 \%$ in most $\mathrm{Pt}$ isotopic ratios.

(3) The accuracy of the Pb-corrected Pt isotope ratios are dependent on the reference values for the $\mathrm{Pb}$ isotope standard SRM981 and, in turn, SRM982 which are considered to be accurate to $\pm 0.036 \%{ }^{66}$

$\mathrm{Pb}$-corrected $\mathrm{Pt}$ isotope ratio measurements using the approach described above are shown in Table 3. For all these measurements, ${ }^{207} \mathrm{~Pb} /{ }^{206} \mathrm{~Pb}_{\mathrm{FC}}$ ratios were within $c a .65 \mathrm{ppm}$ of the SRM981 reference value. Measured Pt isotope ratios are shown in Fig. 1 normalised to the reference composition from Wolff Briche et al. ${ }^{31}$ The most significant offsets in our data from the values of ref. 31 are for the minor isotope ratios ${ }^{190} \mathrm{Pt} /{ }^{194} \mathrm{Pt}$ and ${ }^{192} \mathrm{Pt} /{ }^{194} \mathrm{Pt}$, which show differences of $c a .10 \%$ and $1.7 \%$, respectively. Corrected Pt isotope ratios are in good agreement between the two instruments used in this study, with relative differences of $0.02-0.2 \%$. For all ratios, measured IRMM-010 Pt isotopic compositions from the two instruments in this study are in significantly better agreement with one

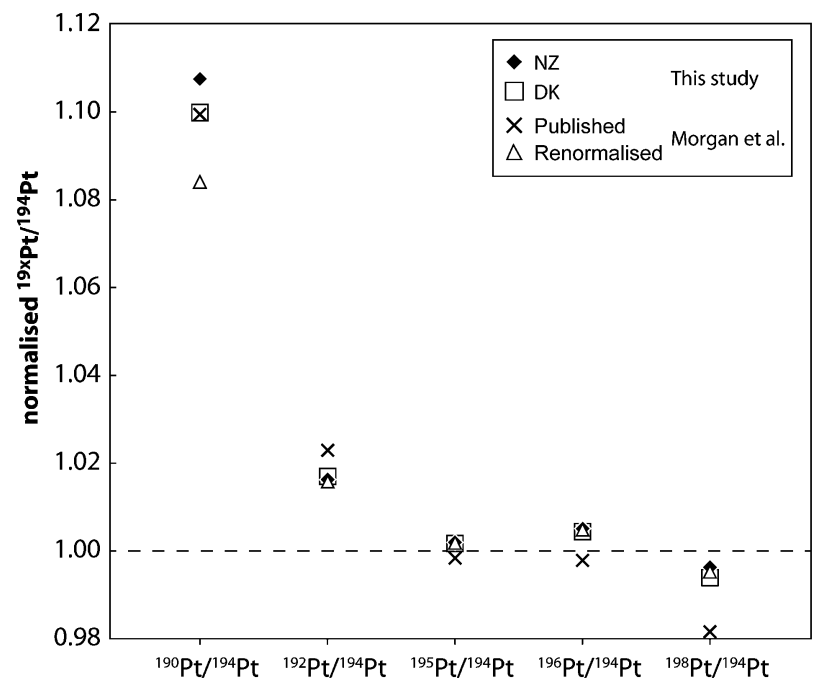

Fig. 1 Natural Pt isotopic composition from Morgan et al. ${ }^{65}$ and Pb-mass-biascorrected MC-ICPMS analysis of the IRMM-010 standard in NZ and DK from this study normalised to the reference composition of Wolff Briche et al. ${ }^{31}$ Morgan et al. data represent published data internally normalised using ${ }^{194} \mathrm{Pt} /{ }^{195} \mathrm{Pt}=$ 0.9744 (crosses), and the same data renormalised to ${ }^{194} \mathrm{Pt} /{ }^{195} \mathrm{Pt}=0.9710$ as determined for natural Pt in this study (triangles).

another and with those of ref. 65 than they are with those of ref. 31. We have thus adopted the mean of the ratios from $\mathrm{NZ}$ and DK in this study as the composition of the IRMM-010 standard.

The analytical uncertainties associated with the $\mathrm{Pb}$-corrected Pt isotope ratios are relatively small compared with the differences from the reference composition. ${ }^{31}$ Estimated combined uncertainties were calculated for corrected ratios according to the uncertainty propagation law. ${ }^{67,68}$ However, the propagated uncertainties are significantly smaller than the variance between $\mathrm{NZ}$ and DK Pt isotope measurements. We have therefore conservatively adopted the $2 \mathrm{sd}$ of the combined NZ and DK values as the uncertainty in the mean of the natural Pt isotope ratios.

Table 3 Natural Pt isotope ratios measured for IRMM-010 obtained by Pb doping, and DS Pt isotope compositions measured by Pb doping and standard bracketing ${ }^{\mathrm{a}}$

\begin{tabular}{|c|c|c|c|c|c|c|c|}
\hline & ${ }^{190} \mathrm{Pt} /{ }^{194} \mathrm{Pt}$ & ${ }^{192} \mathrm{Pt} /{ }^{194} \mathrm{Pt}$ & ${ }^{195} \mathrm{Pt} /{ }^{194} \mathrm{Pt}$ & ${ }^{196} \mathrm{Pt} /{ }^{194} \mathrm{Pt}$ & ${ }^{198} \mathrm{Pt} /{ }^{194} \mathrm{Pt}$ & ${ }^{207} \mathrm{~Pb} /{ }^{206} \mathrm{~Pb}_{\mathrm{FC}}$ & $\delta^{207} \mathrm{~Pb} /{ }^{206} \mathrm{~Pb}(\%)$ \\
\hline \multicolumn{8}{|l|}{ Natural Pt } \\
\hline Wolff Briche et al. & 0.0003561 & 0.023798 & 1.02800 & 0.76719 & 0.22386 & & \\
\hline Morgan et al. & 0.0003921 & 0.024342 & 1.02627 & 0.76562 & 0.21978 & & \\
\hline $\mathrm{NZ}$ & $0.00039435(6)$ & $0.024185(5)$ & $1.02997(1)$ & $0.77116(12)$ & $0.22302(6)$ & $0.91486(1)$ & $-0.036(12)$ \\
\hline DK & $0.00039162(3)$ & $0.024204(2)$ & $1.02973(2)$ & $0.77056(2)$ & $0.22250(1)$ & $0.91495(1)$ & $0.063(8)$ \\
\hline $\mathrm{NZ}+\mathrm{DK}$ & $0.0003930(39)$ & $0.024194(26)$ & $1.02985(35)$ & $0.77086(85)$ & $0.22276(74)$ & $0.91490(13)$ & \\
\hline \multicolumn{8}{|l|}{${ }^{192}$ Pt $-{ }^{198}$ Pt DS } \\
\hline Pb doped NZ & $0.00022(18)$ & $2.03213(21)$ & $0.510597(44)$ & $0.32432(5)$ & $3.9034(9)$ & $0.91486(3)$ & $-0.038(32)$ \\
\hline $\mathrm{Pb}$ doped DK & $0.000211(4)$ & $2.03446(5)$ & $0.510553(9)$ & $0.32407(1)$ & $3.8971(2)$ & $0.91493(3)$ & $0.047(37)$ \\
\hline $\mathrm{NZ}+\mathrm{DK}$ & $0.000215(13)$ & $2.0333(33)$ & $0.510575(62)$ & $0.32420(35)$ & $3.9002(89)$ & $0.91489(11)$ & \\
\hline \multicolumn{8}{|l|}{${ }^{196}$ Pt- ${ }^{198}$ Pt DS } \\
\hline Std bracketing & $0.000161(23)$ & $0.01633(21)$ & $1.76510(93)$ & $13.955(15)$ & $42.732(48)$ & & \\
\hline Average NZ & $0.000178(48)$ & $0.01614(54)$ & $1.76530(58)$ & $13.957(8)$ & $42.734(6)$ & & \\
\hline
\end{tabular}

${ }^{a}$ Uncertainties on last digits are given in parentheses and are based on 2 sd of NZ and DK measurements (natural and ${ }^{192} \mathrm{Pt}-{ }^{198} \mathrm{Pt} \mathrm{DS}$ ) or 2 sd of results from different methods $\left({ }^{196} \mathrm{Pt}-{ }^{198} \mathrm{Pt} \mathrm{DS}\right)$. 
Although the Pt isotope ratios measured in NZ and DK exhibit a closer agreement with one another than those of ref. 31 , differences between the ratios measured in NZ and DK exceed the internal precision of the isotope ratio measurements by a factor of $c a$. 2-8. The differences are greatest for the ratio involving the minor ${ }^{190} \mathrm{Pt}$ isotope and for ratios where the relative mass difference between the numerator and denominator isotope is highest (i.e., ${ }^{198} \mathrm{Pt} /{ }^{194} \mathrm{Pt}$ ). The cause of this discrepancy is not entirely clear. The abundance sensitivity of both of the mass spectrometers used in this study is sufficiently low that tails from abundant Pt isotopes onto less abundant Pt isotopes cannot readily explain the difference. Similarly, the opposite offsets observed for ${ }^{198} \mathrm{Pt} /{ }^{194} \mathrm{Pt}$ and ${ }^{192} \mathrm{Pt} /{ }^{194} \mathrm{Pt}$ between the two laboratories cannot be attributed to contamination or memory from use of the ${ }^{192} \mathrm{Pt}-{ }^{198} \mathrm{Pt}$ DS (the natural measurements were made prior to development of the $\left.{ }^{196} \mathrm{Pt}-{ }^{198} \mathrm{Pt} \mathrm{DS}\right)$. The difference in measured ${ }^{190} \mathrm{Pt} /{ }^{194} \mathrm{Pt}(0.7 \%)$ obtained in the two laboratories is most likely a result of the very small ion beam size of the ${ }^{190} \mathrm{Pt}$ isotope ( $c a .3 \mathrm{mV}$ ). The differences in the other Pt isotope ratios appear to broadly correspond to a mass fractionation relationship as the two sets of ratios lie within 40-180 ppm of a mass fractionation line that passes through the mean composition of both sets of data, implying that subtle differences in the relative mass bias of $\mathrm{Pt}$ and $\mathrm{Pb}$ characterised the two mass spectrometers used in this study.

Relative Pt isotope abundances were calculated from the mean NZ-DK Pt isotope ratios in Table 3. Uncertainties in the abundances were calculated by partitioning ratio uncertainties between the numerator and denominator isotope following an error propagation law and using measured ion beam intensities. ${ }^{68}$ Given that the IRMM-010 Pt isotope standard is accepted as a reference for the natural composition of $\mathrm{Pt}^{8}{ }^{8}$ the new composition for IRMM-010 presented here should also be representative of natural Pt. Our Pt isotopic composition is compared with previously published values for the natural composition of $\mathrm{Pt}$ in Table 1.

We have also recalculated the atomic weight of Pt from the new IRMM-010 isotopic composition presented here using the isotopic masses of ref. 69. We obtain an atomic weight for Pt of $195.08395 \pm 0.00068$. Despite the significant differences in the Pt isotopic composition reported in this study and ref. 31, this new atomic weight is very similar to the value of $195.0844 \pm$ 0.0058 from ref. 31 , although the uncertainty of our new value is significantly smaller than previous estimates (Table 1).

\subsection{Calibration of the platinum double-spikes}

Accurate isotopic determination by the DS technique requires calibration of the DS composition. Several approaches for calibrating the DS are possible. Here, we determined the compositions of the two DS in two ways: (1) externally normalised by doping with $\mathrm{Pb}$ in the same way as described above for the natural Pt isotopic composition of IRMM-010; and (2) external standard-spike bracketing versus IRMM-010. Isotopic abundances for both of the Pt DS used in this study are shown in Table 1 and expressed as ratios in Table 3 . The calibrated DS compositions are not identical to the optimal DS from Rudge et al. (2009), which is due to subtle differences in the pure spike compositions from those used in the DS toolbox (Table 1).

4.2.1 ${ }^{192} \mathrm{Pt}-{ }^{198} \mathrm{Pt}$ double-spike. In the case of the ${ }^{192} \mathrm{Pt}-{ }^{198} \mathrm{Pt}$ $\mathrm{DS}$, Pt-based oxide and nitride interferences on $\mathrm{Pb}$ isotopes were more significant than for IRMM-010 due to the higher abundance of ${ }^{192} \mathrm{Pt}$. Potential levels of interferences on $\mathrm{Pb}$ isotopes were monitored in undoped Pt DS solutions with identical Pt concentrations to the SRM981-Pt DS mixture and were subtracted from $\mathrm{Pb}$-doped analyses. Pb-doped measurements of the ${ }^{192} \mathrm{Pt}-{ }^{198} \mathrm{Pt}$ DS were repeated in NZ and DK. Pbcorrected ${ }^{207} \mathrm{~Pb} /{ }^{206} \mathrm{~Pb}_{\mathrm{FC}}$ ratios in both $\mathrm{NZ}$ and $\mathrm{DK}$ measurements were within $c a$. $50 \mathrm{ppm}$ of the reference value for SRM981. Pt isotopic compositions of this DS measured in NZ and DK are in good agreement, and yielded mean ratios of ${ }^{192} \mathrm{Pt} /{ }^{194} \mathrm{Pt}=2.0333 \pm 0.0033,{ }^{196} \mathrm{Pt} /{ }^{194} \mathrm{Pt}=0.32420 \pm 0.00035$ and ${ }^{198} \mathrm{Pt} /{ }^{194} \mathrm{Pt}=3.9002 \pm 0.0089$ for the three ratios used in the DS calculations, where the uncertainties represent the 2 sd of the NZ and DK isotope measurements (Table 3).
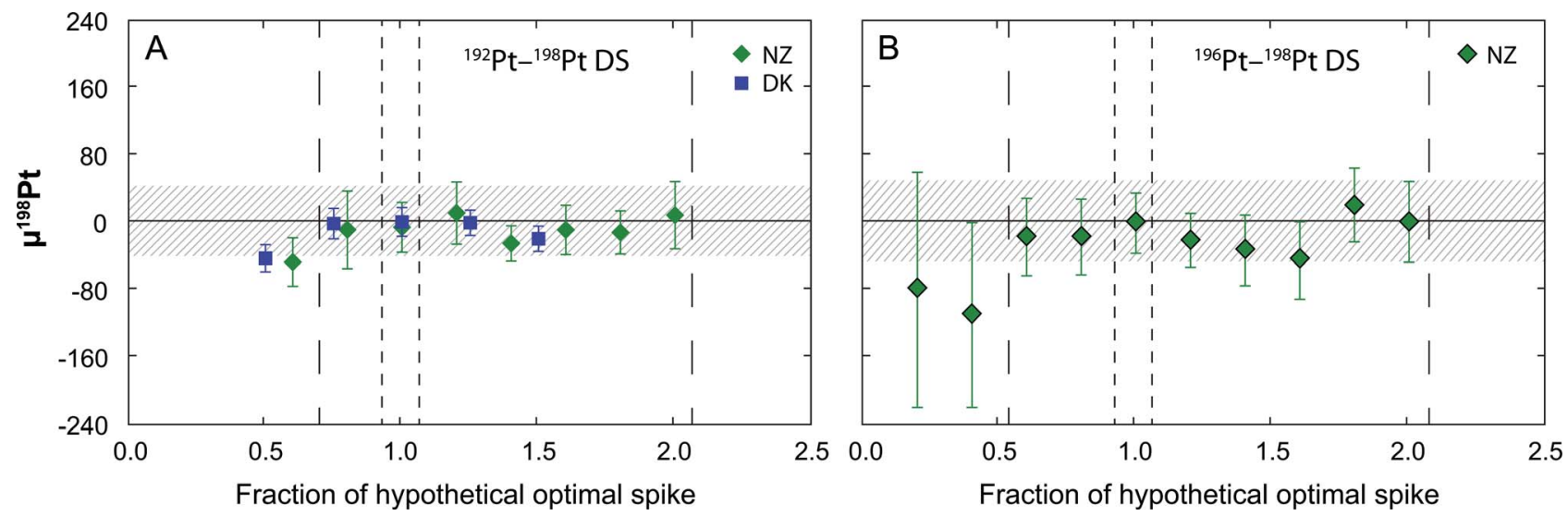

Fig. 2 Plot of $\mu^{198} \mathrm{Pt}$ for a range of sample-spike mixtures expressed as a proportion of the hypothetical optimal sample-spike mixture. (A) Results for the ${ }^{192} \mathrm{Pt}-{ }^{198} \mathrm{Pt}$ double-spike from both NZ and DK. (B) Results for the ${ }^{196} \mathrm{Pt}-{ }^{198} \mathrm{Pt}$ double-spike from NZ only. These results demonstrate that DS-corrected stable Pt isotope ratios are relatively insensitive to the sample-spike ratio. Coarse-dashed vertical lines represent the minimum range over which the calibration appears to be accurate. Finedashed vertical lines represent the approximate range of sample-spike mixtures used in acquiring the data presented elsewhere in this paper. 
4.2.2 ${ }^{196}{ }^{19 t}-{ }^{198} \mathrm{Pt}$ double-spike. The ${ }^{196} \mathrm{Pt}-{ }^{198} \mathrm{Pt}$ DS composition was determined by both Pb doping and IRMM-010 standard-spike bracketing, although these measurements were only carried out in NZ. Both sets of results are very consistent, with compositions agreeing to within $0.04 \%$. The Pt isotope ratios obtained for the ${ }^{196} \mathrm{Pt}-{ }^{198} \mathrm{Pt}$ DS were $\mathrm{Pt} / \mathrm{Pt}=1.76530 \pm 0.00058$, ${ }^{196} \mathrm{Pt} / \mathrm{Pt}=13.957 \pm 0.008$ and ${ }^{198} \mathrm{Pt} / \mathrm{Pt}=42.734 \pm 0.006$, where the uncertainties represent the $2 \mathrm{sd}$ of the ratios obtained by the two different methods.

4.2.3 Accuracy of the double-spike calibrations. The accuracy of the DS calibrations was tested by measuring DS-IRMM010 mixtures in different proportions. DS-corrected results for 8-10 calibration mixtures for both DS, where the mixtures were varied from 0.2-2.0 times the approximate 'optimal' samplespike ratio are shown in Fig. 2. Varying the sample/spike ratios from $\sim 0.6$ to 2.0 times the optimal sample-spike proportion for either DS has no influence on the accuracy of the DS correction, indicating that the two DS have been well calibrated and that a relatively wide window is acceptable for measuring sample-DS mixtures without significant degradation of the results.

\subsection{Internal precision and external reproducibility of double-spike-corrected platinum stable isotope ratios}

Internal precision on DS-corrected Pt isotope ratios varied slightly from session-to-session depending on the stability of the sample introduction systems. However, typical internal (2 se) precision on $\mu^{198} \mathrm{Pt}$ was $c a$. $\pm 30 \mathrm{ppm}$ and $\pm 16 \mathrm{ppm}$ for $\mathrm{NZ}$ and DK analyses, respectively. The improved internal precision
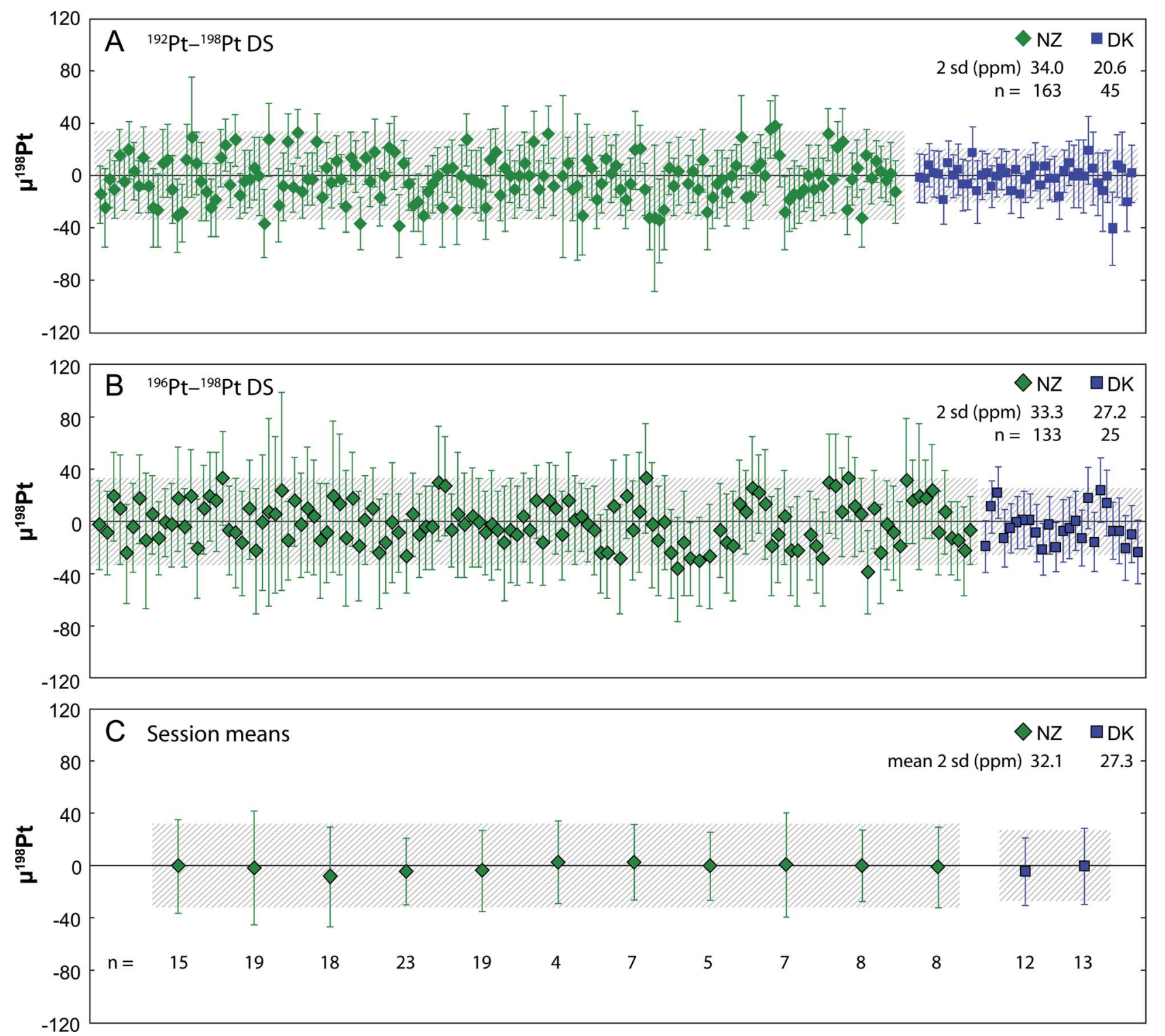

Fig. 3 Reproducibility of double-spiked MC-ICPMS stable Pt isotope measurements from analytical sessions over a period of several months. The first two panels show all analyses from sessions using the ${ }^{192} \mathrm{Pt}-{ }^{198} \mathrm{Pt}(\mathrm{A})$ and ${ }^{196} \mathrm{Pt}-{ }^{198} \mathrm{Pt}(\mathrm{B})$ double-spikes, and error bars representing the 2 se error for each analysis. Hatched fields represent the $\pm 2 \mathrm{sd}$ of all analyses. The bottom panel (C) shows the mean and 2 sd (error bars) for each analytical session for ${ }^{196} \mathrm{Pt}-{ }^{198} \mathrm{Pt} \mathrm{DS}$ data, which is our preferred double-spike. The number of analyses in each session is shown. Hatched fields represent the combined \pm 2 sd of all sessions from each laboratory. 


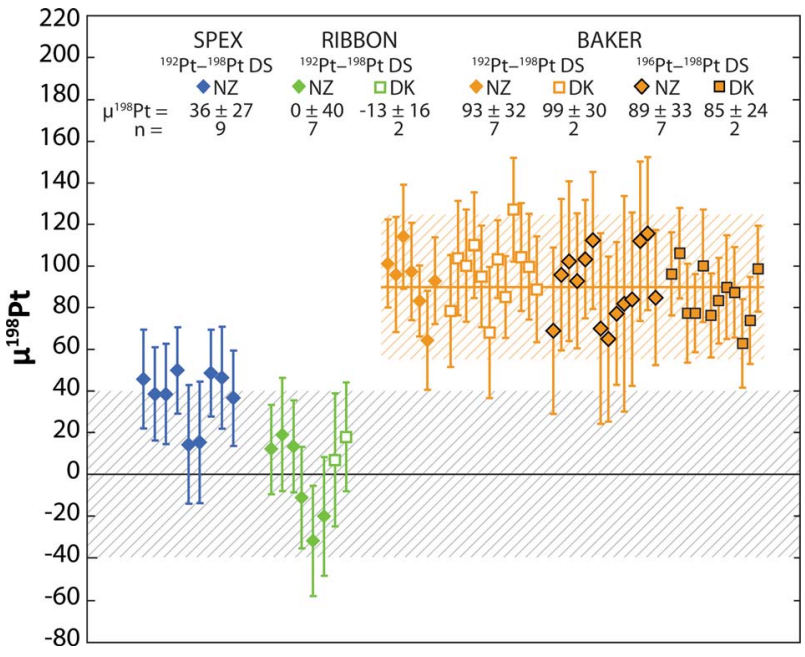

Fig. 4 Measurements of $\mu^{198} \mathrm{Pt}$ in the additional Pt standards described in the text. The Ribbon standard is compositionally identical to the IRMM-010 standard. The Spex standard shows a potential offset from the IRMM-010 composition, although the offset is approximately equal to the reproducibility of the technique. However, the Baker standard show a clear offset from the IRMM-010 standard of $89 \pm 33$ ppm that is reproducible between laboratories and using both types of DS. Grey hatched area represents the reproducibility of the technique. Orange horizontal line and hatched area represent the mean and $2 \mathrm{sd}$, respectively, of al analyses of the Baker standard.

in DK compared with NZ mainly reflects the greater stability of the Apex sample introduction system.

DS-corrected Pt isotope results for IRMM-010 from a number of analytical sessions in both NZ and DK and using both DS are shown in Fig. 3. The external reproducibilities using the two DS are very similar. Within measurement sessions that are typically 1-2 days in length, DS-corrected IRMM-010 measurements have external reproducibilities ( $2 \mathrm{sd}$ ) on $\mu^{198} \mathrm{Pt}$ of $c a . \pm 35 \mathrm{ppm}$ for $\mathrm{NZ}$ analyses and $c a . \pm 25 \mathrm{ppm}$ for DK analyses. External reproducibility over a large number of sessions is similar to reproducibility attained in a single session (Fig. 3.).

Small offsets ( $c a$. 30-50 ppm) were sometimes observed between absolute DS-corrected Pt isotope ratios from different sessions. These offsets might possibly be related to small changes in the abundance sensitivity of the MC-ICPMS, drifts in the relative gains of the Faraday collectors, or a variable component of non-exponential mass bias that is not readily corrected for by the DS method. To account for these variations, we combined the DS correction method with a standard-sample bracketing approach, whereby analyses of the double-spiked standard are repeated throughout the session and alternated with "unknown" analyses. The analyses of "unknowns" are then normalised to the mean value of the bracketing standards. The long-term external reproducibility of these corrected ratios is approximately the same as the within-session reproducibility. The external reproducibility of our technique suggests that mass-dependent Pt stable isotope fractionations $>10 \mathrm{ppm} \mathrm{u}^{-1}$ should be resolvable by this technique. This external reproducibility is comparable to those recently achieved for other non-traditional stable isotope systems such as $\mathrm{Mg}$, Fe and Cd. ${ }^{7,70,71}$

4.3.1 Platinum stable isotope variations in standards. Repeated DS-corrected Pt stable isotope measurements were made of three different Pt element standards (Baker, Ribbon and Spex; Table 3; Fig. 4). ${ }^{192} \mathrm{Pt}^{-198}{ }^{19 t}$ DS-corrected measurements were made of all three standards in both NZ and DK.
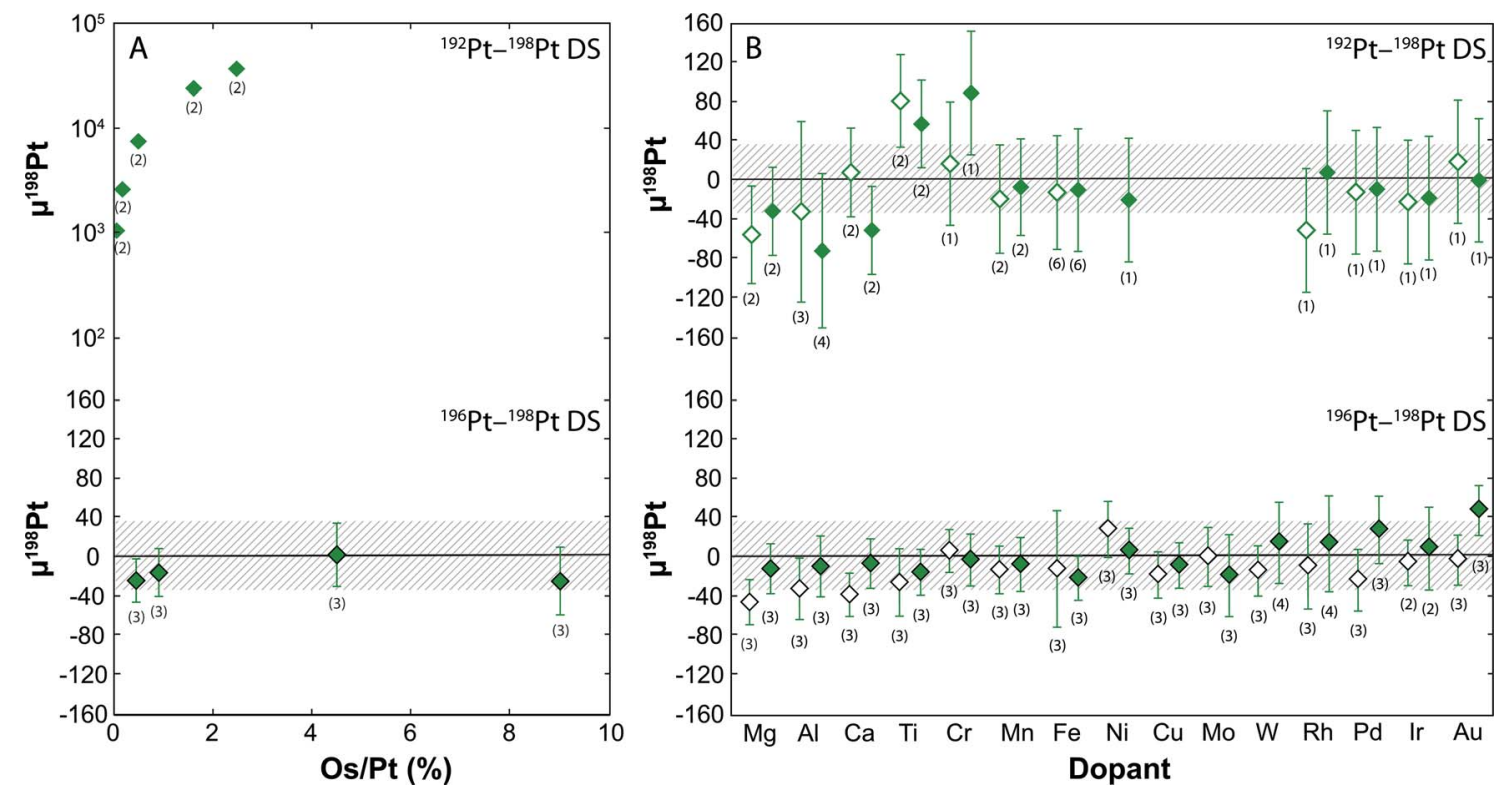

Fig. 5 Results of doped IRMM-010 standard measurements with both double-spikes from NZ. (A) Results of doping tests with a range of Os concentrations up to $10 \%$ of the Pt concentration of the standard. The ${ }^{192} \mathrm{Pt}-{ }^{198} \mathrm{Pt}$ DS-corrected $\mu{ }^{198} \mathrm{Pt}$ data are sensitive to very small amounts of Os contamination (note: data shown have no Os correction applied). However, the ${ }^{196} \mathrm{Pt}-{ }^{198} \mathrm{Pt}$ DS corrected $\mu{ }^{198} \mathrm{Pt}$ appears to be unaffected at the Os doping levels tested here. Hatched areas represent the 2 sd reproducibility of undoped measurements (not shown for the ${ }^{192} \mathrm{Pt}-{ }^{198} \mathrm{Pt} \mathrm{DS}$ ). (B) Results of doping tests with a range of common rock forming elements and siderophile elements for both double-spikes. Solutions of IRMM-010 were doped at 5\% and 10\% of the Pt concentration, represented by hollow and filled symbols, respectively. Hatched areas represent the 2 sd reproducibility of undoped measurements for each DS. 
Measurements using the ${ }^{196} \mathrm{Pt}-{ }^{198} \mathrm{Pt}$ DS were only repeated in both laboratories for the Baker standard. The Spex standard may show a subtle variation from the IRMM-010 standard, however, both Spex and Ribbon are within error of the IRMM010 composition. The Baker standard shows the largest offset in $\mu^{198} \mathrm{Pt}$ of $c a .89 \pm 33$ ppm from IRMM-010, which was reproducibly observed between different analytical sessions in both NZ and DK and using both DS. The Baker standard thus represents a useful secondary standard with a known offset from IRMM-010 to monitor the quality of Pt isotope data within each analytical session. Moreover, these stable Pt isotopic differences between the IRMM-010 and Baker standards suggest that stable isotopic fractionations characterise terrestrial Pt, although it is currently unclear if this is due to variations in the natural Pt source material or those induced during preparation of the Pt metal used to make these standards.

\subsection{Analytical tests}

4.4.1 Spectral matrix effects. Due to the high relative abundances of Os and Os (26.4\% and $41 \%$, respectively), Pt isotope analyses at these masses are likely to be severely impacted by the presence of even small amounts of Os, particularly where one of these two Pt isotopes would be used in the DS calculations. In order to constrain the potential effects of Os isobaric interferences, such as those that might result from imperfect chemical separation of Pt from Os in natural samples, Os doping tests were carried out on DS-IRMM-010 mixtures using both the ${ }^{192} \mathrm{Pt}-{ }^{198} \mathrm{Pt}$ DS and ${ }^{196} \mathrm{Pt}-{ }^{198} \mathrm{Pt}$ DS (Fig. 5A). Os doping levels ranged from $0.1-10 \%$ of the Pt concentration. The ${ }^{196} \mathrm{Pt}-{ }^{198} \mathrm{Pt}$ spike does not use ${ }^{192} \mathrm{Pt}$ in the DS calculations, and is unaffected by the presence of Os even at the highest doping levels. However, DS-corrected Pt isotope data using the ${ }^{192} \mathrm{Pt}-{ }^{198} \mathrm{Pt} \mathrm{DS}$ are highly sensitive to the presence of small amounts of Os. At Os/Pt ratios $>0.1 \%$, DS corrected $\mu^{198} \mathrm{Pt}$ analyses are inaccurate by $>1000 \mathrm{ppm}$. The VUW Nu Plasma collector configuration does not allow for Os/Os to be measured in the same cycle as Pt isotopes, so this approach to calculating Os corrections on ${ }^{190} \mathrm{Pt}$ and ${ }^{192} \mathrm{Pt}$ was tested by measuring Os/Os in a second cycle to determine the Os mass bias, and using Os signal measured in the same cycle as Pt to calculate the magnitude of the corrections. While the Os correction was somewhat effective, it was found to be not sufficiently reliable to enable accurate isotope determinations using the ${ }^{192} \mathrm{Pt}-{ }^{198} \mathrm{Pt} \mathrm{DS}$ in standards even with only very minor amounts of Os contamination (i.e. $\mathrm{Os} / \mathrm{Pt}<0.1 \%$ ).

The influence of Os in ${ }^{192} \mathrm{Pt}-{ }^{198} \mathrm{Pt}$ DS Pt stable isotope measurements may limit the application of this DS to natural samples where Os and Pt may have broadly similar concentrations. Whilst chemical separation techniques can be used to quantitatively separate Os from Pt, and the presence of Os can at least be monitored using non-overlapping masses (e.g., Os), it is difficult to rigorously correct for the presence of even minor amounts of Os. As such, the ${ }^{196} \mathrm{Pt}-{ }^{198} \mathrm{Pt}$ DS is our preferred Pt DS for use with terrestrial samples. Furthermore, a recent study has shown that, due to their relatively large thermal neutron capture cross sections, Pt isotope compositions can be significantly altered by neutron capture in iron meteorites with long cosmic ray exposure ages. ${ }^{34}$ These effects are significantly larger for ${ }^{192} \mathrm{Pt}$ than ${ }^{196} \mathrm{Pt}$. Therefore, the ${ }^{196} \mathrm{Pt}-{ }^{198} \mathrm{Pt}$ DS is also our preferred DS for use with extraterrestrial samples.

4.4.2 Non-spectral matrix effects. Non-spectral matrix effects from elements that have no direct isobaric interferences with the element of interest can arise from a range of possible factors and can have an influence on instrumental mass bias. ${ }^{57}$ In order to investigate potential non-spectral matrix effects, a range of elemental doping tests were performed whereby solutions of optimally double-spiked IRMM-010 were doped at levels of $5-10 \%$ of the Pt concentration, including a range of common rock-forming elements ( $\mathrm{Mg}, \mathrm{Al}, \mathrm{Ca}, \mathrm{Ti}, \mathrm{Cr}, \mathrm{Mn}, \mathrm{Fe}, \mathrm{Ni}$ and $\mathrm{Cu}$ ) as well some siderophile elements (Mo, W, Rh, Pd, Ir and Au). The

Table 4 Results of elemental doping tests to assess potential matrix effects. Optimally spiked IRMM-010 samples were doped with a range of elements ${ }^{a}$

\begin{tabular}{|c|c|c|c|c|c|c|c|}
\hline \multirow[b]{2}{*}{ Dopant } & \multirow{2}{*}{$\begin{array}{l}\text { Doping } \\
\text { level }\end{array}$} & \multicolumn{3}{|c|}{${ }^{192} \mathrm{Pt}-{ }^{198} \mathrm{Pt}$ DS } & \multicolumn{3}{|c|}{${ }^{196} \mathrm{Pt}-{ }^{198} \mathrm{Pt}$ DS } \\
\hline & & $\mu^{198} \mathrm{Pt}$ & $2 \mathrm{sd}$ & $n$ & $\mu^{198} \mathrm{Pt}$ & $2 \mathrm{sd}$ & $n$ \\
\hline Undoped & & 0.0 & 63 & 39 & 0.0 & 43.0 & 49 \\
\hline \multirow[t]{2}{*}{$\mathrm{Mg}$} & $5 \%$ & -54.5 & 38.4 & 2 & -45.0 & 23.0 & 3 \\
\hline & $10 \%$ & -30.5 & 31.9 & 2 & -11.0 & 25.2 & 3 \\
\hline \multirow[t]{2}{*}{$\mathrm{Al}$} & $5 \%$ & -31.0 & 86.5 & 3 & -31.3 & 31.3 & 3 \\
\hline & $10 \%$ & -70.8 & 72.1 & 4 & -8.7 & 30.9 & 3 \\
\hline \multirow[t]{2}{*}{$\mathrm{Ca}$} & $5 \%$ & 9.0 & 32.6 & 2 & -37.3 & 22.3 & 3 \\
\hline & $10 \%$ & -50.0 & 31.8 & 2 & -5.7 & 24.9 & 3 \\
\hline \multirow[t]{2}{*}{$\mathrm{Ti}$} & $5 \%$ & 82.0 & 35.1 & 2 & -25.0 & 34.2 & 3 \\
\hline & $10 \%$ & 58.5 & 31.6 & 2 & -14.7 & 23.0 & 3 \\
\hline \multirow[t]{2}{*}{$\mathrm{Cr}$} & $5 \%$ & 18.0 & 63.0 & 1 & 7.0 & 21.6 & 3 \\
\hline & $10 \%$ & 90.0 & 63.0 & 1 & -2.0 & 26.4 & 3 \\
\hline \multirow[t]{2}{*}{ Mn } & $5 \%$ & -18.0 & 45.3 & 2 & -12.3 & 24.0 & 3 \\
\hline & $10 \%$ & -6.0 & 38.0 & 2 & -6.7 & 27.4 & 3 \\
\hline \multirow[t]{2}{*}{$\mathrm{Fe}$} & $5 \%$ & -11.3 & 48.6 & 6 & -11.3 & 59.2 & 3 \\
\hline & $10 \%$ & -8.8 & 53.9 & 6 & -20.7 & 22.4 & 3 \\
\hline \multirow[t]{2}{*}{$\mathrm{Ni}$} & $5 \%$ & & & & 29.0 & 28.4 & 3 \\
\hline & $10 \%$ & -19.0 & 63.0 & 1 & 7.0 & 23.2 & 3 \\
\hline \multirow[t]{2}{*}{$\mathrm{Cu}$} & $5 \%$ & & & & -17.3 & 23.4 & 3 \\
\hline & $10 \%$ & & & & -7.7 & 23.0 & 3 \\
\hline \multirow[t]{2}{*}{ Mo } & $5 \%$ & & & & 1.0 & 29.9 & 3 \\
\hline & $10 \%$ & & & & -18.0 & 41.5 & 3 \\
\hline \multirow[t]{2}{*}{ W } & $5 \%$ & & & & -13.3 & 25.4 & 3 \\
\hline & $10 \%$ & & & & 15.3 & 41.0 & 4 \\
\hline \multirow[t]{2}{*}{$\mathrm{Rh}$} & $5 \%$ & -50.0 & 63.0 & 1 & -8.7 & 43.3 & 3 \\
\hline & $10 \%$ & 9.0 & 63.0 & 1 & 14.5 & 48.8 & 4 \\
\hline \multirow[t]{2}{*}{$\mathrm{Pd}$} & $5 \%$ & -11.0 & 63.0 & 1 & -22.7 & 31.3 & 3 \\
\hline & $10 \%$ & -8.0 & 63.0 & 1 & 28.0 & 34.5 & 3 \\
\hline \multirow[t]{2}{*}{ lr } & $5 \%$ & -21.0 & 63.0 & 1 & -5.0 & 23.1 & 2 \\
\hline & $10 \%$ & -17.0 & 63.0 & 1 & 9.5 & 42.0 & 2 \\
\hline \multirow[t]{5}{*}{$\mathrm{Au}$} & $5 \%$ & 20.0 & 63.0 & 1 & -2.3 & 25.5 & 3 \\
\hline & $10 \%$ & 1.0 & 63.0 & 1 & 48.0 & 25.5 & 3 \\
\hline & $0.1 \%$ & -2.0 & 135.8 & 2 & & & \\
\hline & $0.2 \%$ & 15.0 & 65.1 & 2 & & & \\
\hline & $0.5 \%$ & 117.0 & 45.3 & 2 & -23.3 & 22.1 & 3 \\
\hline \multirow[t]{4}{*}{ Os } & $0.9 \%$ & 584.0 & 11.3 & 2 & -15.3 & 24.2 & 3 \\
\hline & $1.8 \%$ & 1063.0 & & 1 & & & \\
\hline & $4.5 \%$ & 3022.5 & 58.0 & 2 & 2.7 & 32.0 & 3 \\
\hline & $9.0 \%$ & & & & -24.0 & 34.2 & 3 \\
\hline
\end{tabular}

${ }^{a}$ Uncertainties are propagated 2 sd errors. Italicised 2 sd values are where only one analysis was made and the session $2 \mathrm{sd}$ is taken as the uncertainty. No Os interference correction for ${ }^{192} \mathrm{Pt}-{ }^{198} \mathrm{Pt}$ DS data was applied. 

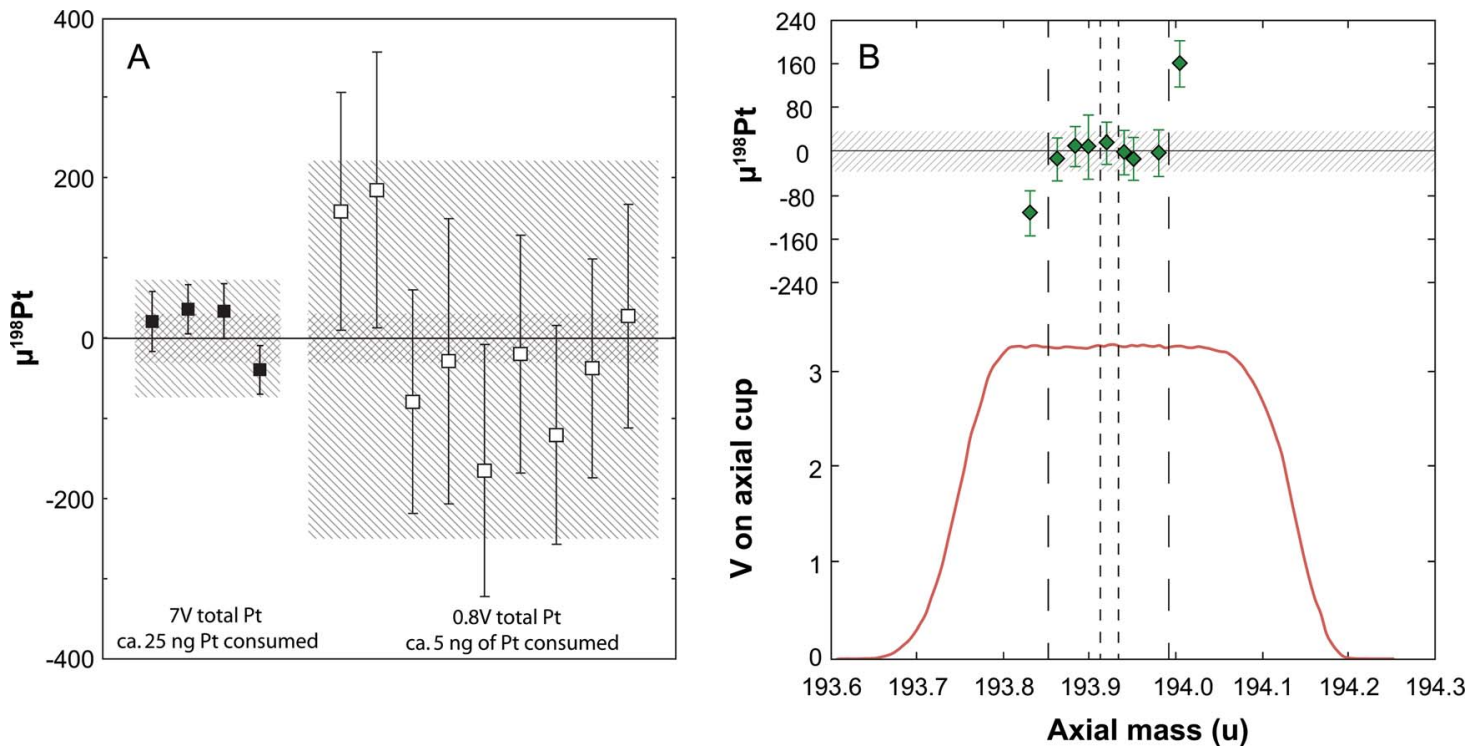

Fig. 6 (A) Repeat measurements of IRMM-010 with the ${ }^{192} \mathrm{Pt}-{ }^{198} \mathrm{Pt}$ DS at $7 \mathrm{~V}$ (filled symbols) and ${ }^{196} \mathrm{Pt}-{ }^{198} \mathrm{Pt} \mathrm{DS}$ at $0.8 \mathrm{~V}$ total signal on Pt (usually $24 \mathrm{~V}$ at typical measurement concentration). Internal and external precision are concomitantly larger (ca. 35 ppm and 73 ppm, respectively, for $7 \vee$ signal, and ca. 150 ppm and 240 ppm, respectively, for $0.8 \mathrm{~V}$ signal). Hatched area represents the 2 sd error for each group of samples. Cross-hatched areas represent the reproducibility of the technique using typical measurement concentrations. (B) Peak scan from the Nu Plasma MC-ICPMS in low resolution, with measurements of $\mu^{198} \mathrm{Pt}$ for IRMM-010 using the ${ }^{196} \mathrm{Pt}-{ }^{198} \mathrm{Pt}$ DS superimposed at their respective analysis peak positions (expressed in unified atomic mass units, $\mathrm{u}$ ). The coarse dashed vertical lines represent the range over which the peak position had no effect. The fine dashed vertical lines represent the range of axial masses of normal measurements in this study. The hatched area represents the reproducibility of the technique.

results of these tests are shown in Fig. 5B. The ${ }^{192} \mathrm{Pt}-{ }^{198} \mathrm{Pt}$ DScorrected data for IRMM-010 doped with this range of elements at levels of $5-10 \%$ are largely within analytical uncertainty of undoped IRMM-010, although $\mathrm{Ti}, \mathrm{Cr}$ and $\mathrm{Al}$ may have a moderate $(c a .<100 \mathrm{ppm})$ effect on the accuracy of Pt stable isotope measurements using the ${ }^{192} \mathrm{Pt}-{ }^{198} \mathrm{Pt}$ double-spike (Table 4; Fig. 5b). Element-doped IRMM-010 measured using the ${ }^{196} \mathrm{Pt}-{ }^{198} \mathrm{Pt}$ DS yielded Pt stable isotope ratios that were almost all within analytical precision of undoped IRMM-010. However, IRMM-010 doped with Au yielded a mean $\mu^{198} \mathrm{Pt}$ of $48.0 \pm 27.5 \mathrm{ppm}$, which might reflect a small hydride interference (AuH) on ${ }^{198} \mathrm{Pt}$.

These elemental-doping tests show that for most elements tested, impurities of up to $10 \%$ of the Pt concentration do not significantly compromise the accuracy of the DS-corrected Pt isotope ratios. These results provide some constraints on the efficiency of chemical separation procedures that will be required to separate sufficiently pure Pt from natural samples for accurate Pt stable isotope analysis.

4.4.3 Effect of Pt signal intensity. To test the influence of signal intensity on DS-corrected Pt stable isotope measurements, DS-IRMM-010 mixtures were measured at $c a .30 \%$ and $3 \%$ of the typical Pt concentration analysed in this study (i.e. $7 \mathrm{~V}$ and $0.8 \mathrm{~V}$ total Pt signal compared with $24 \mathrm{~V}$ at the typical Pt concentration; Fig. 6A). At $7 \mathrm{~V}$ total Pt signal, the DS-corrected Pt stable isotope ratios were within error of those measured at optimal intensities, although the internal precision and external reproducibility were slightly higher ( $c a .35 \mathrm{ppm}$ and 70 $\mathrm{ppm}$, respectively). At $0.8 \mathrm{~V}$ total $\mathrm{Pt}$ signal, the internal precision and external reproducibility were much larger (ca. $150 \mathrm{ppm}$ and
$240 \mathrm{ppm}$, respectively). These data show that it will be possible to measure the Pt stable isotopic composition $\left(\mu^{198} \mathrm{Pt}\right)$ of natural samples to $\pm 70 \mathrm{ppm}$ on sample of $25 \mathrm{ng}$ of $\mathrm{Pt}$, and to $\pm 240 \mathrm{ppm}$ on a sample of $5 \mathrm{ng}$ of Pt.

4.4.4 Effect of peak position. When operated in low-resolution mode, the Nu Plasma MC-ICPMS produces flat-topped peaks that are $c a .0 .39 \mathrm{u}$ wide (full width at half maximum) for Pt masses. We tested the potential effects on Pt stable isotope ratio measurements that might result from imperfect alignment of the Pt isotope peaks, peak centering, and peak flatness by measuring DS-corrected Pt isotope ratios on IRMM-010 in different peak measurement positions. Analyses were made by manually setting the axial mass to a range of positions up to 0.1 amu either side of the peak centre and performing analyses of IRMM-010 using the ${ }^{196} \mathrm{Pt}-{ }^{198} \mathrm{Pt}$ DS. These tests showed that DScorrected Pt isotope ratios were within analytical precision of those at the peak centre even when positioned up to $c a . \pm 0.075$ $\mathrm{u}(c a . \pm 25 \%$ of the plateau width) from the peak centre (Fig. $6 \mathrm{~B})$. This range is significantly broader than any peak drift that is likely to occur during an analytical session and, thus, subtle changes in peak position are unlikely to have any effect on the accuracy of our measurements.

\section{Conclusions}

We have established a DS method for the precise measurement of Pt stable isotopes by MC-ICPMS using both ${ }^{192} \mathrm{Pt}-{ }^{198} \mathrm{Pt}$ and ${ }^{196} \mathrm{Pt}-{ }^{198} \mathrm{Pt}$ double-spikes. The latter spike is preferable for application to natural terrestrial and extraterrestrial samples as it is (relatively) insensitive to the presence of minor amounts of 
Os and any cosmogenic effects that can affect Pt isotopes in meteorite samples with long cosmic ray exposure ages. ${ }^{\mathbf{3 4 3 5}}$ Repeated measurements of Pt standards show that an external precision of $c a . \pm 10 \mathrm{ppm} \mathrm{u}^{-1}$ is routinely achievable using this technique, even in the presence of significant amounts of matrix elements. One of the analysed Pt standards clearly shows an offset from the IRMM-010 Pt isotope standard that suggest terrestrial stable Pt isotope variations exist that exceed our analytical uncertainty.

While Pt stable isotope variations in nature might be expect to be small, this new isotopic tracer may hold considerable promise for use in a range of areas, including; (1) provenancing of global Pt sources and precious metal archeological artefacts; (2) understanding the global Pt geochemical cycle and, in particular, using redox-controlled Pt stable isotope variations in marine authigenic sediments to trace and understand past changes in ocean and atmospheric chemistry; (3) metal-silicate and liquid-solid metal fractionation associated with the differentiation of asteroids and terrestrial planets in the young Solar System.

\section{Acknowledgements}

We thank André Poirier for providing a segment of platinum ribbon, and Vickie Bennett for supplying additional PGE solutions. The authors also wish to thank $\mathrm{H}$. Williams and one anonymous reviewer for their constructive comments on the manuscript. This research was supported by a Royal Society of New Zealand Marsden Grant to $\mathrm{MH}$ and JB. JC is funded by a VUW PhD scholarship. The Centre for Star and Planet Formation is funded by the Danish National Research Foundation and the University of Copenhagen's Programme of Excellence.

\section{References}

1 A. N. Halliday, D.-C. Lee, J. N. Christensen, M. Rehkämper, W. Yi, X. Luo, C. M. Hall, C. J. Ballentine, T. Pettke and C. Stirling, Geochim. Cosmochim. Acta, 1998, 62, 919-940.

2 F. Albarède, Rev. Mineral. Geochem., 2004, 55, 409-427.

3 A. D. Anbar, Rev. Mineral. Geochem., 2004, 55, 429-454.

4 B. L. Beard and C. M. Johnson, Rev. Mineral. Geochem., 2004, 55, 319-357.

5 E. D. Young and A. Galy, Rev. Mineral. Geochem., 2004, 55, 197-230.

6 A. D. Anbar and O. Rouxel, Annu. Rev. Earth Planet. Sci., 2007, 35, 717-746.

7 M. Bizzarro, C. Paton, K. Larsen, M. Schiller, A. Trinquier and D. Ulfbeck, J. Anal. At. Spectrom., 2011, 26, 565.

8 M. Berglund and M. E. Wieser, Pure Appl. Chem., 2011, 83, 397-410.

9 A. D. Brandon and R. J. Walker, Earth Planet. Sci. Lett., 2005, 232, 211-225.

10 F. A. Cotton and G. Wilkinson, Advanced Inorganic Chemistry: A Comprehensive Text, Interscience Publishers, 1966.

11 K. Righter, Annu. Rev. Earth Planet. Sci., 2003, 31, 135-174. $12 \mathrm{~J}$. Lorand, A. Luguet and O. Alard, Elements, 2008, 4, 247-252. 13 V. F. Hodge, M. Stallard, M. Koide and E. D. Goldberg, Earth Planet. Sci. Lett., 1985, 72, 158-162.
14 D. C. Colodner, E. A. Boyle and J. M. Edmond, Anal. Chem., 1993, 65, 1419-1425.

15 P. Halbach, C. Kriete, B. Prause and D. Puteanus, Chem. Geol., 1989, 76, 95-106.

16 E. D. Goldberg and M. Koide, Mar. Chem., 1990, 30, 249-257.

17 V. Banakar, J. Hein, R. Rajani and A. Chodankar, J. Earth Syst. Sci., 2007, 116, 3-13.

18 J. Roberts, A. Thomson and W. E. Cohn, in Progress in Nucleic Acid Research and Molecular Biology, Academic Press, 1979, vol. 22, pp. 71-133.

19 M. J. Abrams and B. A. Murrer, Science, 1993, 261, 725-730.

20 Z. Guo, P. J. Sadler and A. Sykes, in Advances in Inorganic Chemistry, Academic Press, 1999, vol. 49, pp. 183-306.

21 Y. Ho, S. C. F. Au-Yeung and K. K. W. To, Med. Res. Rev., 2003, 23, 633-655.

22 R. M. Heck and R. J. Farrauto, Catalytic Air Pollution Control: Commercial Technology, Van Nostrand Reinhold, New York, USA, 1995.

23 J. Kašpar, P. Fornasiero and N. Hickey, Catal. Today, 2003, 77, 419-449.

24 K. H. Ek, G. M. Morrison and S. Rauch, Sci. Total Environ., 2004, 334-335, 21-38.

25 J. C. Ely, C. R. Neal, C. F. Kulpa, M. A. Schneegurt, J. A. Seidler and J. C. Jain, Environ. Sci. Technol., 2001, 35, 3816-3822.

26 F. A. White, T. L. Collins and F. M. Rourke, Phys. Rev., 1956, 101, 1786-1791.

27 T. B. Coplen, J. Phys. Chem. Ref. Data, 1997, 26, 1239-1253.

28 P. D. P. Taylor, S. Valkiers, P. De Bièvre, U. Flegel and T. Kruck, Proceedings of the Second Alfred O. Nier Symposium on Inorganic Mass Spectrometry, Durango, Colorado, 9-12 May 1994, pp. 90-94.

29 C. S. J. Briche, P. D. P. Taylor and P. De Bièvre, Anal. Chem., 1997, 69, 791-793.

30 K. Hattori, D. P. Menagh and T. J. S. Cole, Anal. Chem., 1998, 70, 4100-4103.

31 C. Wolff Briche, A. Held, M. Berglund, P. De Bièvre and P. Taylor, Anal. Chim. Acta, 2002, 460, 41-47.

32 H. Emteborg, X. Tian, M. Ostermann, M. Berglund and F. C. Adams, J. Anal. At. Spectrom., 2000, 15, 239-246.

33 X. Tian, H. Emteborg and F. C. Adams, J. Anal. At. Spectrom., 1999, 14, 1807-1814.

34 T. S. Kruijer, M. Fischer-Gödde, T. Kleine, P. Sprung, I. Leya and R. Wieler, Earth Planet. Sci. Lett., 2013, 361, 162-172.

35 N. Wittig, M. Humayun, A. Brandon, S. Huang and I. Leya, Earth Planet. Sci. Lett., 2013, 361, 152-161.

36 A. D. Anbar, K. A. Knab and J. Barling, Anal. Chem., 2001, 73, 1425-1431.

37 T. Arnold, M. Schönbächler, M. Rehkämper, S. Dong, F.-J. Zhao, G. J. D. Kirk, B. J. Coles and D. J. Weiss, Anal. Bioanal. Chem., 2010, 398, 3115-3125.

38 J. Baker, D. Peate, T. Waight and C. Meyzen, Chem. Geol., 2004, 211, 275-303.

39 B. L. Beard and C. M. Johnson, Geochim. Cosmochim. Acta, 1999, 63, 1653-1660.

40 B. L. Beard, C. M. Johnson, L. Cox, H. Sun, K. H. Nealson and C. Aguilar, Science, 1999, 285, 1889-1892. 
41 K. Dideriksen, J. Baker and S. Stipp, Geochim. Cosmochim. Acta, 2006, 70, 118-132.

42 O. Eugster, F. Tera and G. J. Wasserburg, J. Geophys. Res., 1969, 74, 3897-3908.

43 K. Gopalan, D. Macdougall and C. Macisaac, Int. J. Mass Spectrom., 2006, 248, 9-16.

44 J. L. Mann and W. R. Kelly, Rapid Commun. Mass Spectrom., 2005, 19, 3429-3441.

45 R. Markey, J. L. Hannah, J. W. Morgan and H. J. Stein, Chem. Geol., 2003, 200, 395-406.

46 S. Ripperger and M. Rehkämper, Geochim. Cosmochim. Acta, 2007, 71, 631-642.

47 K. Rosman, Geochim. Cosmochim. Acta, 1972, 36, 801-819.

48 R. Schoenberg, S. Zink, M. Staubwasser and F. von Blanckenburg, Chem. Geol., 2008, 249, 294-306.

49 C. Siebert, T. F. Nägler and J. D. Kramers, Geochem., Geophys., Geosyst., 2001, 2, 16.

50 M. H. Dodson, J. Sci. Instrum., 1963, 40, 289-295.

51 W. Compston and V. M. Oversby, J. Geophys. Res., 1969, 74, 4338-4348.

52 B. Hamelin, G. Manhes, F. Albarede and C. J. Allègre, Geochim. Cosmochim. Acta, 1985, 49, 173-182.

53 R. Powell, J. Woodhead and J. Hergt, Chem. Geol., 1998, 148, 95-104.

54 S. J. G. Galer, Chem. Geol., 1999, 157, 255-274.

55 C. M. Johnson and B. L. Beard, Int. J. Mass Spectrom., 1999, 193, 87-99.

56 J. F. Rudge, B. C. Reynolds and B. Bourdon, Chem. Geol., 2009, 265, 420-431.

57 F. Albarède and B. Beard, Rev. Mineral. Geochem., 2004, 55 113-152.
58 R. D. Russell, J. Geophys. Res., 1971, 76, 4949-4955.

59 P. De Biévre, M. Gallet, N. E. Holden and I. L. Barnes, J. Phys. Chem. Ref. Data, 1984, 13, 809-891.

60 K. J. R. Rosman and P. D. P. Taylor, J. Phys. Chem. Ref. Data, 1998, 27, 1275-1287.

61 A. Scherstén, T. Elliott, C. Hawkesworth, S. Russell and J. Masarik, Earth Planet. Sci. Lett., 2006, 241, 530542.

62 S. B. Shirey and R. J. Walker, Annu. Rev. Earth Planet. Sci., 1998, 26, 423-500.

63 C. Paton, J. Hellstrom, B. Paul, J. Woodhead and J. Hergt, J. Anal. At. Spectrom., 2011, 26, 2508-2518.

64 C. M. Johnson, B. L. Beard and F. Albarède, Rev. Mineral. Geochem., 2004, 55, 1-24.

65 J. W. Morgan, R. J. Walker, M. F. Horan, E. S. Beary and A. J. Naldrett, Geochim. Cosmochim. Acta, 2002, 66, 273290.

66 E. Catanzaro, T. Murphy, W. Shields and E. Garner, J. Res. Natl. Bur. Stand., Sect. A, 1968, 72, 261-267.

67 JCGM 100:2008 GUM 1995 with minor corrections, Evaluation of Measurement Data-Guide to the Expression of Uncertainty in Measurements, Joint Committee for Guides in Metrology (BIPM, IEC, IFCC, ILAC, ISO, IUPAC, IUPAP and OIML).

68 R. Williams, Vienna, Austria, 2010.

69 G. Audi, O. Bersillon, J. Blachot and A. Wapstra, Nucl. Phys. A, 2003, 729, 3-128.

70 M.-A. Millet, J. A. Baker and C. E. Payne, Chem. Geol., 2012, 304-305, 18-25.

71 A. D. Schmitt, S. J. G. Galer and W. Abouchami, J. Anal. At. Spectrom., 2009, 24, 1079-1088. 


\section{Appendix C}

\section{Reprint of Chemical Geology paper}

Chapter 3 of this thesis has been published in the journal Chemical Geo-

logy. A reprint of the article is included here with the page numbers and formatting as published. The article can also be found online using the DOI: 10.1016/j.chemgeo.2013.11.009. 


\title{
Platinum stable isotope analysis of geological standard reference materials by double-spike MC-ICPMS
}

\author{
J.B. Creech ${ }^{\text {a,c, } *}$, J.A. Baker ${ }^{b}$, M.R. Handler ${ }^{\text {a }}$, M. Bizzarro ${ }^{c}$ \\ a School of Geography, Environment and Earth Sciences, Victoria University of Wellington, P.O. Box 600, Wellington, New Zealand \\ b School of Environment, The University of Auckland, Private Bag 92019, Auckland, New Zealand \\ c Centre for Star and Planet Formation, Natural History Museum of Denmark, University of Copenhagen, DK-1350 Copenhagen, Denmark
}

\section{A R T I C L E I N F O}

\section{Article history:}

Received 24 July 2013

Received in revised form 13 November 2013

Accepted 14 November 2013

Available online 27 November 2013

Editor: L. Reisberg

\section{Keywords:}

Platinum

Stable isotopes

Double-spike

Standard reference materials

\begin{abstract}
A B S T R A C T
We report a method for the chemical purification of Pt from geological materials by ion-exchange chromatography for subsequent Pt stable isotope analysis by multiple-collector inductively coupled plasma mass spectrometry (MC-ICPMS) using a ${ }^{196} \mathrm{Pt}-{ }^{198} \mathrm{Pt}$ double-spike to correct for instrumental mass bias. Double-spiking of samples was carried out prior to digestion and chemical separation to correct for any mass-dependent fractionation that may occur due to incomplete recovery of Pt. Samples were digested using a NiS fire assay method, which pre-concentrates Pt into a metallic bead that is readily dissolved in acid in preparation for anionexchange chemistry. Pt was recovered from anion-exchange resin in concentrated $\mathrm{HNO}_{3}$ acid after elution of matrix elements, including the other platinum group elements (PGE), in dilute $\mathrm{HCl}$ and $\mathrm{HNO}_{3}$ acids. The separation method has been calibrated using a precious metal standard solution doped with a range of synthetic matrices and results in Pt yields of $\geq 90 \%$ with purity of $\geq 95 \%$. Using this chemical separation technique, we have separated Pt from 11 international geological standard reference materials comprising of PGE ores, mantle rocks, igneous rocks and one sample from the Cretaceous-Paleogene boundary layer. Pt concentrations in these samples range from ca. $5 \mathrm{ng} \mathrm{g}^{-1}$ to $4 \mu \mathrm{g} \mathrm{g}^{-1}$. This analytical method has been shown to have an external reproducibility on $\delta^{198} \mathrm{Pt}$ (permil difference in the ${ }^{198} \mathrm{Pt} /{ }^{194} \mathrm{Pt}$ ratio from the IRMM-010 standard) of \pm 0.040 (2 sd) on Pt solution standards (Creech et al., 2013, J. Anal. At. Spectrom. 28, 853-865). The reproducibility in natural samples is evaluated by processing multiple replicates of four standard reference materials, and is conservatively taken to be ca. \pm 0.088 ( $2 \mathrm{sd}$ ). Pt stable isotope data for the full set of reference materials have a range of $\delta^{198} \mathrm{Pt}$ values with offsets of up to $0.4 \%$ from the IRMM-010 standard, which are readily resolved with this technique. These results demonstrate the potential of the Pt isotope system as a tracer in geochemical systems.
\end{abstract}

(c) 2013 Elsevier B.V. All rights reserved.

\section{Introduction}

Platinum (Pt) has six naturally occurring isotopes $\left({ }^{190} \mathrm{Pt},{ }^{192} \mathrm{Pt},{ }^{194} \mathrm{Pt}\right.$, ${ }^{195} \mathrm{Pt},{ }^{196} \mathrm{Pt}$ and ${ }^{198} \mathrm{Pt}$ ). Like all platinum group elements (PGE; Ru, Rh, $\mathrm{Pd}$, Os, Ir and Pt), Pt is highly siderophile and prefers bonds with metal over silicon and oxygen and, as such, is concentrated in the cores of terrestrial planets. The PGE also show some chalcophile behaviour, and in the conditions found in the crust and mantle are strongly partitioned into sulphide minerals. Platinum can exist in a range of oxidation states, but in the Earth principally occurs as $\mathrm{Pt}^{0}, \mathrm{Pt}^{2+}$ and $\mathrm{Pt}^{4+}$ (Cotton and Wilkinson, 1966). The relatively large mass difference (2\%) between the abundant heavy and light isotopes of Pt, coupled with its variable redox states in the Earth's core, mantle and surficial environments, and the large differences in Pt abundances that characterise Earth's major geochemical reservoirs suggest that Pt may exhibit significant stable isotope variations. However, to-date there have been no

\footnotetext{
* Corresponding author. Fax: +6444635186.

E-mail address: john.creech@snm.ku.dk (J.B. Creech).
}

published studies of Pt stable isotope variations in natural terrestrial samples.

We have developed an improved anion-exchange method for chemical separation of Pt from geological materials. Using this method, Pt has been separated from a range of international geological standard reference materials, and their Pt stable isotopic compositions were then determined using a recently developed method for precise Pt stable isotope measurement by double-spike (DS) multiple collector inductively coupled plasma mass spectrometry (MC-ICPMS) (Creech et al., 2013). These results reveal significant differences in Pt stable isotopic composition amongst this suite of samples and provide reference Pt stable isotope data for standards for use in future applications of this technique.

\section{Samples}

A suite of 11 international geological standard reference materials were selected based on availability and published Pt contents. The Pt abundances of these samples span practically the entire range found 
on Earth. A short description of each of the analysed materials is given here and summarised in Table 1.

The standard reference materials are considered in groups as follows:

(a) Pt ore samples: SARM-76 is from the Merensky Reef Ore of the Bushveld Igneous Complex in South Africa, which contains most of the world's known reserves of PGE. The PGE are hosted in extensive sulphide layers within the unit, which have an emplacement age of ca. $2.06 \mathrm{Ga}$ (Walraven et al., 1990). SARM-76 is distributed by MINTEK. PTA-1 is a platiniferous black sand from the Tulameen River area of British Columbia, Canada, which is described as being essentially a magnetite concentrate, containing an abundant Pt-Fe alloy (Faye and Sutarno, 1976). The Tulameen ultramafic-gabbro complex has an emplacement age of ca. $186 \mathrm{Ma}$ (Findlay, 1969). The PTA-1 reference material is distributed by the CANMET Canadian Certified Reference Materials Project (CCRMP). WPR-1 is an altered ultramafic igneous rock from the Wellgreen Complex, Yukon, Canada, which is also distributed by CANMET-CCRMP. The Wellgreen Complex is part of the greater Quill Creek ultramafic complex, which has an emplacement age of 232 Ma (Marcantonio et al., 1994). Pt in WPR-1 is thought to be hosted in base metal sulphides (Barkov et al., 2002; Meisel and Moser, 2004), platinum group minerals (Barkov et al., 2002) and tellurides (CANMET-CCRMP reference certificate). The three Pt ore standards have Pt concentrations ranging from 285 to $3590 \mathrm{ng} \mathrm{g}^{-1}$ (Table 1 ).

(b) Mantle-derived samples: PCC-1 is a peridotite standard from California, USA, distributed by the US Geological Survey (USGS), although supplies of this material are now exhausted. JP-1 is a peridotite from the Horoman peridotite, Hokkaido Island, Japan. The petrogenesis of the Horoman peridotite is complicated, but it is considered to have been emplaced ca. $23 \mathrm{Ma}$ (Takazawa et al., 1999) and to represent former sub-oceanic lithosphere (Takazawa et al., 1992, 1996, 2000).JP-1 is distributed by the Geological Society of Japan (GSJ). OKUM is an ultramafic komatiite distributed by Geosciences Laboratories, that was sampled from Serpentine Mountain, Ontario, Canada. This area is located at the western end of the Abitibi Greenstone Belt, and was emplaced into the crust ca. 2.7 Ga (Houlé et al., 2009). PGE in these rocks are found as Ni-Cu-PGE minerals (Houlé et al., 2009). Although OKUM is technically an igneous sample, komatiites are considered to be large degree partial melts of the underlying mantle (e.g. Nisbet and Walker, 1982) and their compositions are expected to reflect the mantle source. Platinum concentrations in the mantle-derived samples range from ca. 5-12 $\mathrm{ng} \mathrm{g}^{-1}$ (Table 1).

(c) Igneous samples: SARM-6 is a dunite from an ultrabasic pipe in the Bushveld Complex, South Africa provided by MINTEK. As with SARM-76 described above, this unit was emplaced ca. $2.06 \mathrm{Ga}$, and PGE are hosted in sulphide minerals. LK-NIP is a dolerite from the Nipigon Diabase, Ontario, Canada distributed by Geosciences Laboratories. The Nipigon diabase sills were intruded into the crust ca. $1100 \mathrm{Ma}$ (Hart and MacDonald, 2007; Heaman et al., 2007). DNC-1 is a dolerite from North Carolina, USA, distributed by the USGS. BIR-1 is a tholeiitic basalt from a lava flow near Reykjavik, Iceland, distributed by the USGS. These igneous samples have Pt concentrations ranging from ca. $4 \mathrm{ng} \mathrm{g}^{-1}$ in BIR-1 to $39 \mathrm{ng} \mathrm{g}^{-1}$ in SARM-6 (Table 1).

(d) Other samples: FC-1 was collected from the Fish Clay at Stevns Klint in Denmark. FC-1 was sampled from a sedimentary layer that is stratigraphically located at the Cretaceous-Paleogene boundary and has elevated levels of Ir, due to the putative meteorite impact at that time (Alvarez et al., 1980). The FC-1 standard is distributed by the University of Copenhagen, and has a Pt concentration of $38 \mathrm{ng} \mathrm{g}^{-1}$ (Table 1 ).

\section{Materials and methods}

\subsection{Reagents, standard solutions and spikes}

Analytical reagent grade nitric $\left(\mathrm{HNO}_{3}\right)$ and hydrochloric $(\mathrm{HCl})$ acids were purified by sub-boiling distillation in quartz or Teflon stills, respectively, prior to use in anion-exchange chemistry. Acid used in the digestion steps (including aqua regia) and all solutions for MC-ICPMS analysis were prepared from Optima ${ }^{\mathrm{TM}}$ grade $\mathrm{HNO}_{3}$ and $\mathrm{HCl}$ acids, which were used as supplied without further purification. Acid dilutions and all water rinses were performed with ultrapure $(>18 \mathrm{M} \Omega \cdot \mathrm{cm})$ water.

In the initial stages of technique development, a mixed 'precious metals' standard from Alfa Aesar supplier (SpecPure plasma standard, $100 \mu \mathrm{g} \mathrm{m}^{-1}$ in $20 \% \mathrm{HCl}$, Lot \# 012912A) was used, containing Ru, Rh, Pd, Re, Os, Ir, Pt and Au. For performing column calibrations, several synthetic rock mixtures were prepared in order to have compositions approximating those of a peridotite, chondrite, and iron meteorite (Table S1) by mixing single-element standard solutions and the precious metal standard solution at the appropriate concentration levels. Mixtures containing all of the elements present in the synthetic rock mixtures were also prepared from standard solutions at concentrations of $1 \mu \mathrm{g} \mathrm{mL} L^{-1}$ and $1 \mathrm{ng} \mathrm{mL}^{-1}$ and used as a bracketing standards in ICP-MS measurements of ion-exchange fractions for the construction of elemental elution profiles.

The Pt isotope standard IRMM-010 was purchased from the Institute for Reference Materials and Measurements, Belgium. The supplied Pt wire segments were carefully weighed and then cleaned prior to digestion by sequentially rinsing in ultrapure water, analytical grade methanol and dilute $(5 \%) \mathrm{HNO}_{3}$. The cleaned IRMM-010 was then digested in aqua regia $\left(\mathrm{HCl}: \mathrm{HNO}_{3}\right.$ prepared in a volume ratio of 3:1) in a Savillex Teflon beaker. The IRMM-010 solution was then evaporated, converted to chloride in concentrated $\mathrm{HCl}$, evaporated again, and finally redissolved in $20 \% \mathrm{HCl}$. From this, a $60 \mathrm{ng} \mathrm{mL}^{-1}$ solution of

Table 1

Details of the standard reference materials analysed in this study.

\begin{tabular}{|c|c|c|c|c|c|c|}
\hline Group & Provider & Standard & Lot/batch & Type & Locality & $\begin{array}{l}\text { Pt conc. }{ }^{a} \\
\left(\mu g g^{-1}\right)\end{array}$ \\
\hline \multirow[t]{3}{*}{ Ore } & CANMET-CCRMP & PTA-1 & & Platiniferous black sand & Tulameen River, British Columbia & 3.05 \\
\hline & CANMET-CCRMP & WPR-1 & & Ultramafic igneous rock & Wellgreen complex, Yukon, Canada & 0.285 \\
\hline & MINTEK & SARM-76 & & PGM ore & Merensky Reef, Bushveld Complex, South Africa & 3.59 \\
\hline \multirow[t]{3}{*}{ Mantle } & Geosciences Lab. & OKUM & & Ultramafic komatiite & Serpentine Mountain, Ontario, Canada & 0.01144 \\
\hline & GSJ & JP-1 & Split 3 pos 42 & Peridotite & Horoman peridotite, Horoman, Hokkaido, Japan & 0.0049 \\
\hline & USGS & PCC-1 & Split 34 pos 3 & Peridotite & East Austin Creek, Sonoma County, California, USA & 0.008 \\
\hline \multirow[t]{4}{*}{ Igneous } & Geosciences Lab. & LK-NIP & & Diabase & Nipigon diabase, Ontario, Canada & 0.01343 \\
\hline & MINTEK & SARM-6 & & Dunite & Ultrabasic pipe, Bushveld Complex, South Africa & 0.039 \\
\hline & USGS & BIR-1 & Lot 0036 & Tholeiitic basalt & Reykjavik, Iceland & 0.0043 \\
\hline & USGS & DNC-1 & Lot 0667 & Dolerite & Braggtown Quarry, North Carolina & 0.0368 \\
\hline Other & Univ. of Copenhagen & FC-1 & & Fish Clay & Stevns Klint, Denmark & 0.038 \\
\hline
\end{tabular}

${ }^{a}$ Concentrations for standard reference materials were taken from the GeoRem database and references therein. 
IRMM-010 in $0.5 \mathrm{M} \mathrm{HCl}$ was prepared and double-spiked for use as a bracketing standard for MC-ICPMS isotopic measurements (Creech et al., 2013).

The Pt DS used for the isotopic analyses was prepared using two single isotope spikes ( ${ }^{196} \mathrm{Pt}$ and ${ }^{198} \mathrm{Pt}$ ) obtained from the Oak Ridge National Laboratory, USA. Full details of the design and calibration of the DS are given in Creech et al. (2013).

\subsection{Laboratory environment}

Sample digestions were carried out in an ultra-clean laboratory at Victoria University of Wellington. All Pt separation work was conducted in class 10 laminar flow hoods situated in a class 100 clean laboratory.

Savillex ${ }^{\mathrm{TM}}$ Teflon beakers were used for all sample digestions and collection of eluents from chemistry. Beakers were cleaned thoroughly between uses by the following process: pre-cleaning by refluxing with aqua regia overnight on a hotplate at $120^{\circ} \mathrm{C}$; batch cleaning in $6 \mathrm{M}$ $\mathrm{HCl}$ (AR grade) in a large glass beaker overnight on a hotplate at $50{ }^{\circ} \mathrm{C}$; batch cleaning in $7 \mathrm{M} \mathrm{HNO}_{3}$ (AR grade) in a large glass beaker overnight on a hotplate at $50{ }^{\circ} \mathrm{C}$; two periods of overnight refluxing with aqua regia, prepared from Optima ${ }^{\mathrm{TM}}$ grade $\mathrm{HNO}_{3}$ and $\mathrm{HCl}$ acids, on a hotplate at $120^{\circ} \mathrm{C}$. Beakers were thoroughly rinsed with ultrapure water between each of these steps.

Crushing of samples or NiS beads was performed using an agate mortar and pestle, which was cleaned thoroughly between uses by rinsing with methanol and ultrapure water and by grinding pure quartz grains.

\subsection{Sample digestion}

Digestion of the standards was carried out using the NiS fire assay technique. This method is commonly used in PGE work, and involves melting the sample in the presence of $\mathrm{Ni}$ and $\mathrm{S}$, which form a metallic NiS bead into which the PGE are sequestered. The NiS bead can then be physically removed from the silicate slag and readily digested in acid. By eliminating most of the rock matrix elements, this approach has the advantage of pre-concentrating the PGE, but can have relatively high blanks when compared with acid digestion techniques (Rehkämper and Halliday, 1997). Most NiS fire assay procedures use relatively large amounts of sample, which has been found to reduce the influence of 'nugget' effects and improve the external reproducibility of PGE concentration measurements. A number of procedures for NiS fire assay have been published, with subtle differences in flux amounts and fusion time (e.g. Rehkämper and Halliday, 1997; Ravizza and Pyle, 1997; Gros et al., 2002; Morcelli et al., 2004; Savard et al., 2010). The procedure used here largely follows Morcelli et al. (2004), although it includes the addition of silica as has been adopted in some other studies (e.g. Gros et al., 2002).

Powdered material of the terrestrial standards was weighed directly into porcelain crucibles ( $50 \mathrm{~mL}$, medium wall, Lomb Scientific). The NiS fire assay charges typically comprised $15 \mathrm{~g}$ of sample. When less sample was used due to high Pt concentrations or limited availability of material, the sample weight was made up to $15 \mathrm{~g}$ using pure silica. Procedural blanks for the NiS fire assay technique were assessed by digesting $15 \mathrm{~g}$ of pure silica with no sample.

The samples in the porcelain crucibles were then thoroughly mixed with $10 \mathrm{~g}$ of sodium carbonate, $20 \mathrm{~g}$ of sodium tetraborate, $1 \mathrm{~g}$ of nickel powder and $0.75 \mathrm{~g}$ of sulphur. For samples that were spiked prior to NiS digestion, Pt DS was added to the mixture in the crucible from a solution of the ${ }^{196} \mathrm{Pt}-{ }^{198} \mathrm{Pt}$ DS in the appropriate volume for a ratio of $55 \% \mathrm{Pt}$ from DS and 45\% natural Pt, and allowed to dry. Crucibles were then placed in a furnace and fused at $950{ }^{\circ} \mathrm{C}$ for $30 \mathrm{~min}$, and at $1050{ }^{\circ} \mathrm{C}$ for a further $30 \mathrm{~min}$ (Morcelli et al., 2004). The crucibles were removed from the furnace and left to cool at room temperature. Once cooled, the crucibles were physically broken and NiS beads were carefully removed from the silicate slag. The NiS beads were then weighed and crushed into smaller fragments in an agate mortar and then weighed again before being transferred to Savillex Teflon beakers for acid dissolution.

The NiS beads were dissolved in ca. $30 \mathrm{~mL}$ of concentrated $\mathrm{HCl}$ on a hotplate at $120^{\circ} \mathrm{C}$ with lids off, and then left to evaporate. Once dry, another $\mathrm{ca} .30 \mathrm{~mL}$ of concentrated $\mathrm{HCl}$ was added to the samples, and the beakers were again placed on a hotplate at $120{ }^{\circ} \mathrm{C}$ with their lids off for $2-3 \mathrm{~h}$. The beakers were removed from the hotplate, and a volume of concentrated $\mathrm{HNO}_{3}$ approximately one-third of that of the remaining $\mathrm{HCl}$ volume was added to produce aqua regia. The beakers were then closed and placed on a hotplate at $120^{\circ} \mathrm{C}$ overnight, following which the samples were typically fully in solution.

The samples were then evaporated to dryness, taken up in ca. 5-10 $\mathrm{mL}$ of concentrated $\mathrm{HCl}$ to convert them to chloride form, evaporated to dryness again, and finally taken up in $10 \mathrm{~mL}$ of $0.5 \mathrm{M} \mathrm{HCl}$ in preparation for anion-exchange chemistry.

\subsection{Anion-exchange chemistry}

Chemical separation of the element of interest is desirable in order to increase analytical sensitivity and to reduce potential isobaric or molecular interferences or matrix effects during isotope analysis by MCICPMS. Ion-exchange chromatographic techniques have been widely employed for this purpose. Methods for the separation of PGEs from rocks have previously been developed (e.g., Rehkämper and Halliday, 1997; Pearson and Woodland, 2000). However, the focus of these methods has generally been the recovery of all the PGE for the determination of their concentrations. In this study, we developed a technique for the recovery and purification solely of Pt at a level suitable for precise determination of isotopic compositions by MC-ICPMS.

Prior to loading in columns, Bio-Rad® ${ }^{\circledR}$ AG1-X8 anion exchange resin (100-200 mesh, chloride form) was sequentially cleaned by shaking in ultrapure water, $6 \mathrm{M} \mathrm{HCl}$, ultrapure water, $4 \mathrm{M} \mathrm{HNO}_{3}$ and then ultrapure water, with the liquid decanted off between each step (Rehkämper and Halliday, 1997). All ion-exchange separations were performed using Eichrom polypropylene columns with a $2 \mathrm{~mL}$ resin capacity and a $20 \mathrm{~mL}$ reservoir. Approximately $1 \mathrm{~mL}$ of fresh BioRad® AG1-X8 anion exchange resin was loaded as a slurry into the columns and allowed to gravity settle. The resin bed was cleaned prior to use with the following sequence of reagents: $20 \mathrm{~mL}$ of $0.8 \mathrm{M} \mathrm{HNO}_{3}, 10 \mathrm{~mL}$ of $11 \mathrm{M} \mathrm{HCl}, 25 \mathrm{~mL}$ of $13.5 \mathrm{M} \mathrm{HNO}_{3}$ and $40 \mathrm{~mL}$ of $6 \mathrm{M} \mathrm{HCl}$ (for reconversion to chloride form) (Rehkämper and Halliday, 1997).

Immediately prior to loading, columns were equilibrated using $40 \mathrm{~mL}$ of $0.5 \mathrm{M} \mathrm{HCl}$. The elution sequence used is shown in Fig. 1. Samples were loaded onto the columns in $10 \mathrm{~mL}$ of $0.5 \mathrm{M} \mathrm{HCl}$. Loading was followed by a $40 \mathrm{~mL}$ rinse with $0.5 \mathrm{M} \mathrm{HCl}$, during which virtually all of the major cationic constituents of the sample and some of the weakly retained PGE (Rh, Ru and Ir) were eluted. This was followed by elution of $20 \mathrm{~mL}$ of $1 \mathrm{M} \mathrm{HCl}$, which elutes any remaining weakly retained PGE. Following these $\mathrm{HCl}$ elutions, $20 \mathrm{~mL}$ of $0.8 \mathrm{M} \mathrm{HNO}_{3}$ was added to elute transition metals such as Zn (Rehkämper and Halliday, 1997; Pearson and Woodland, 2000). This was followed by elution of $60 \mathrm{~mL}$ of $11 \mathrm{M} \mathrm{HCl}$, which elutes Re, Pd and any remaining Ru from the resin. The Pt fraction was finally eluted with $40 \mathrm{~mL}$ of $13.5 \mathrm{M} \mathrm{HNO}_{3}$. Pt cuts were collected in Savillex Teflon beakers and evaporated to dryness on a hotplate at $120^{\circ} \mathrm{C}$. During elution tests, cuts from every step were collected in $20 \mathrm{~mL}$ fractions, apart from the $0.5 \mathrm{M} \mathrm{HCl}$ wash which was collected in one $40 \mathrm{~mL}$ fraction.

To monitor procedural blanks, a blank sample solution was processed through anion-exchange chemistry with each batch of samples.

\subsection{Column calibration by ICP-MS}

Prior to analysis, dried fractions from the column calibration experiments were converted to chloride form by taking them up in concentrated $\mathrm{HCl}$ and evaporating before being redissolved in $5 \mathrm{~mL}$ of $0.5 \mathrm{M}$ $\mathrm{HCl}$ and left on a hotplate for $2-3 \mathrm{~h}$ before analysis by ICP-MS. An 


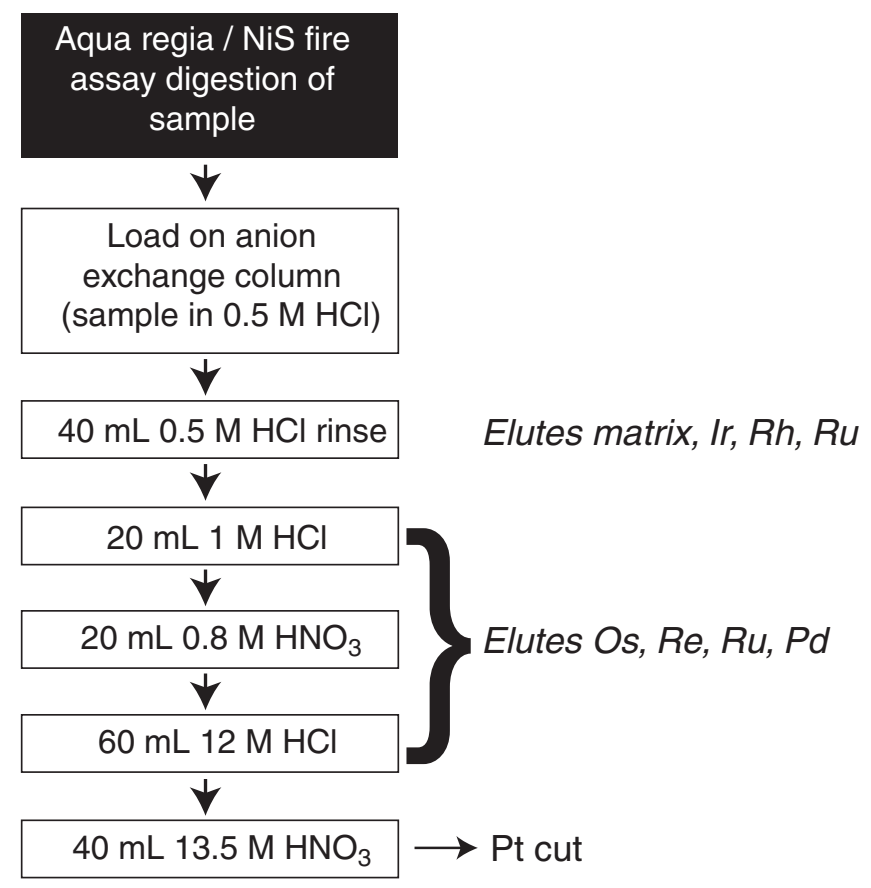

Fig. 1. Flow chart illustrating the Pt separation scheme.

Agilent 7500CS quadrupole ICP-MS with a standard cross-flow nebuliser was used to measure the column fractions. The following masses were monitored for column calibration experiments with the precious metal standard: ${ }^{99} \mathrm{Ru},{ }^{103} \mathrm{Rh},{ }^{105} \mathrm{Pd},{ }^{185} \mathrm{Re},{ }^{189} \mathrm{Os},{ }^{191} \mathrm{Ir}$ and

${ }^{195} \mathrm{Pt}$. Analyses of the column calibration cuts were bracketed with analyses of a gravimetrically prepared solution of the same precious metal standard with a concentration of $1 \mathrm{ng} \mathrm{mL}^{-1}$.

For column calibration experiments with synthetic rock matrices, the following masses were monitored: ${ }^{23} \mathrm{Na},{ }^{25} \mathrm{Mg},{ }^{27} \mathrm{Al},{ }^{29} \mathrm{Si},{ }^{33} \mathrm{~S},{ }^{43} \mathrm{Ca}$, ${ }^{47} \mathrm{Ti},{ }^{52} \mathrm{Cr},{ }^{55} \mathrm{Mn},{ }^{57} \mathrm{Fe},{ }^{59} \mathrm{Co},{ }^{60} \mathrm{Ni},{ }^{63} \mathrm{Cu},{ }^{66} \mathrm{Zn},{ }^{71} \mathrm{Ga},{ }^{72} \mathrm{Ge},{ }^{75} \mathrm{As},{ }^{95} \mathrm{Mo}$, ${ }^{99} \mathrm{Ru},{ }^{103} \mathrm{Rh},{ }^{105} \mathrm{Pd},{ }^{118} \mathrm{Sn},{ }^{121} \mathrm{Sb},{ }^{182} \mathrm{~W},{ }^{185} \mathrm{Re},{ }^{189} \mathrm{Os},{ }^{191} \mathrm{Ir},{ }^{195} \mathrm{Pt}$ and ${ }^{197} \mathrm{Au}$. Analyses of the column calibration cuts were bracketed with standard solutions containing all of these elements $\left(1 \mu \mathrm{gL}^{-1}\right.$ and $1 \mathrm{ng} \mathrm{mL}{ }^{-1}$ ) that were gravimetrically prepared from single element standards. Semi-quantitative concentrations of the relevant elements were calculated for each fraction relative to the measured signal intensities for the standard. Elution curves constructed with these data are estimated to be accurate to $\pm 5 \%$.

\subsection{Platinum stable isotope analysis by double-spike MC-ICPMS}

A ${ }^{196} \mathrm{Pt}-{ }^{198} \mathrm{Pt}$ DS was used to correct for instrumental mass fractionation during MC-ICPMS analysis. The development of the Pt DS and mass spectrometry protocols are described in detail by Creech et al. (2013).

Samples for isotopic analysis were either spiked before sample digestion or prior to ion-exchange chemistry. Samples that were spiked prior to ion-exchange chemistry were refluxed in aqua regia on a hotplate at $120^{\circ} \mathrm{C}$ overnight to ensure complete sample-spike equilibration. These sample-spike mixtures were subsequently evaporated to dryness, reconverted to chloride form by evaporating concentrated $\mathrm{HCl}$, and finally redissolved in $10 \mathrm{~mL}$ of $0.5 \mathrm{M} \mathrm{HCl}$ for loading onto columns.

In order to destroy any potential organic interferences arising from resin that may have been stripped from the resin bed by the large volume of concentrated $\mathrm{HNO}_{3}$ that was used to elute Pt, the Pt cuts were treated after chemistry by the following steps. Pt cuts were evaporated to dryness, refluxed in aqua regia on a hotplate overnight, evaporated to dryness again, and then refluxed overnight in a mixture of $\mathrm{HNO}_{3}-\mathrm{H}_{2} \mathrm{O}_{2}$. The Pt cuts were then evaporated to dryness, reconverted to chloride form by evaporating concentrated $\mathrm{HCl}$, and finally redissolved in an appropriate volume of $0.5 \mathrm{M} \mathrm{HCl}$ resulting in a Pt solution concentration of approximately $60 \mathrm{ng} \mathrm{mL}^{-1}$. Samples were refluxed on a hotplate for at least 2 to $3 \mathrm{~h}$ prior to analysis.

Pt stable isotope measurements were carried out using a Nu Plasma MC-ICPMS at Victoria University of Wellington, New Zealand. The Nu Plasma MC-ICPMS was operated in low-resolution mode, with a mass resolution of $\sim 1840$ (M/ $\Delta \mathrm{M}$; peak edge width from 5 to $95 \%$ full peak height). A Nu Instruments DSN-100 desolvation nebuliser system with an ESI PFA micro-flow nebuliser was used for Pt solution introduction to the mass spectrometer. Measurements were made on $0.5 \mathrm{M} \mathrm{HCl}$ solutions with ca. $60 \mathrm{ng} \mathrm{mL}^{-1}$ of natural Pt. The sample uptake rate was ca. $80 \mathrm{~mL} \mathrm{~min}^{-1}$, and the sensitivity of the instrument was typically ca. $280 \mathrm{~V} \mathrm{ppm}^{-1}$ total Pt under these conditions. Each analysis comprised $100 \times 8 \mathrm{~s}$ integrations, corresponding to $13.3 \mathrm{~min}$ of data acquisition, and consumed ca. $80 \mathrm{ng}$ of natural Pt. Baselines were obtained by measuring on-peak for $100 \times 8 \mathrm{~s}$ integrations prior to each analysis. A washout time of ca. $30 \mathrm{~min}$ was applied between analyses and baseline measurements. Using this approach, background levels were typically $<1 \mathrm{mV}$ on all Pt isotopes.

Data reduction was performed off-line using the freely available data reduction software Iolite, which runs within Igor Pro (Paton et al., 2011). The DS calculations were performed algebraically based on the equations presented in Rudge et al. (2009) using a new procedure incorporated within Iolite (Creech and Paul, submitted for publication). We report Pt stable isotope results in terms of $\delta^{198} \mathrm{Pt}$, representing the permil deviation of the ${ }^{198} \mathrm{Pt} /{ }^{194} \mathrm{Pt}$ ratio in the sample from the same ratio for the IRMM-010 Pt isotope standard. This technique has been shown to have an external reproducibility on $\delta^{198} \mathrm{Pt}$ measurements of Pt solution standards of \pm 0.04 (i.e. $\pm 0.01 \mathrm{amu}^{-1}$; Creech et al., 2013).

\section{Results and discussion}

\subsection{Chemical separation and recovery of Pt}

Results of elution tests for the chemical separation of Pt are shown in Fig. 2. Despite undertaking these column calibration experiments on a wide range of synthetically prepared materials, there is little difference in the elution profiles of the matrix elements and PGE. In all of the elution tests, almost all of the matrix elements are entirely eluted from the column prior to Pt collection, passing through the column in the loading acid. The exceptions to this are $\mathrm{Zn}$, which is eluted in $0.8 \mathrm{M} \mathrm{HNO}_{3}$, minor amounts of $\mathrm{Si}$ and $\mathrm{Sn}$ that were occasionally found in other cuts, and Au which was found to also be completely recovered in the $13.5 \mathrm{M} \mathrm{HNO}_{3}$ cut.

$\mathrm{Rh}$ is only very weakly retained on the anion resin, and is almost entirely eluted in the sample loading solution, with any remaining $\mathrm{Rh}$ being eluted in the $0.5 \mathrm{M} \mathrm{HCl}$ wash. Ru and Ir are also relatively weakly retained on the column. Previous studies have used oxidants (e.g., bromine water, chlorine gas) to convert Ir to Ir(IV), which is more strongly retained on the resin, and can be subsequently eluted with the addition of a reducing agent. However, given that we did not use such a step, Ir is only weakly retained and is largely eluted during the $0.5 \mathrm{M} \mathrm{HCl}$ rinse after loading and, hence, is presumably mostly in the reduced state (i.e., $\operatorname{Ir}(\mathrm{III}))$. In some cases, traces of Ir were found to be present in the $0.8 \mathrm{M} \mathrm{HNO}_{3}$ or $11 \mathrm{M} \mathrm{HCl}$ cuts and, thus, some Ir was likely present as $\operatorname{Ir}(\mathrm{IV})$. The same is also probably the case for $\mathrm{Ru}$, which is mostly eluted during the post-loading wash, but also shows a second peak in the $11 \mathrm{M}$ $\mathrm{HCl}$ cuts. During elution tests with the precious metal solution, 80-90\% of the Os was eluted in $0.8 \mathrm{M} \mathrm{HNO}_{3}$. However, in all of the elution tests with a synthetic rock matrix, virtually no Os was present. Given that multiple evaporations of the solutions were conducted in the preparation of these samples and the highly volatile nature of Os, it is likely that Os was lost during those steps. Re was largely eluted in the first $20 \mathrm{~mL}$ of the $11 \mathrm{M} \mathrm{HCl}$ cut, with the remainder being eluted in the next $20 \mathrm{~mL}$ of $11 \mathrm{M} \mathrm{HCl}$. Pd is less easily eluted than Re in $11 \mathrm{M} \mathrm{HCl}$, 
(a) Precious metals std. $\mathrm{Ru}-\mathrm{Rh}-\mathrm{Pd}-\mathrm{Re}-\mathrm{Os}-\mathrm{Ir}-\mathrm{Pt}$
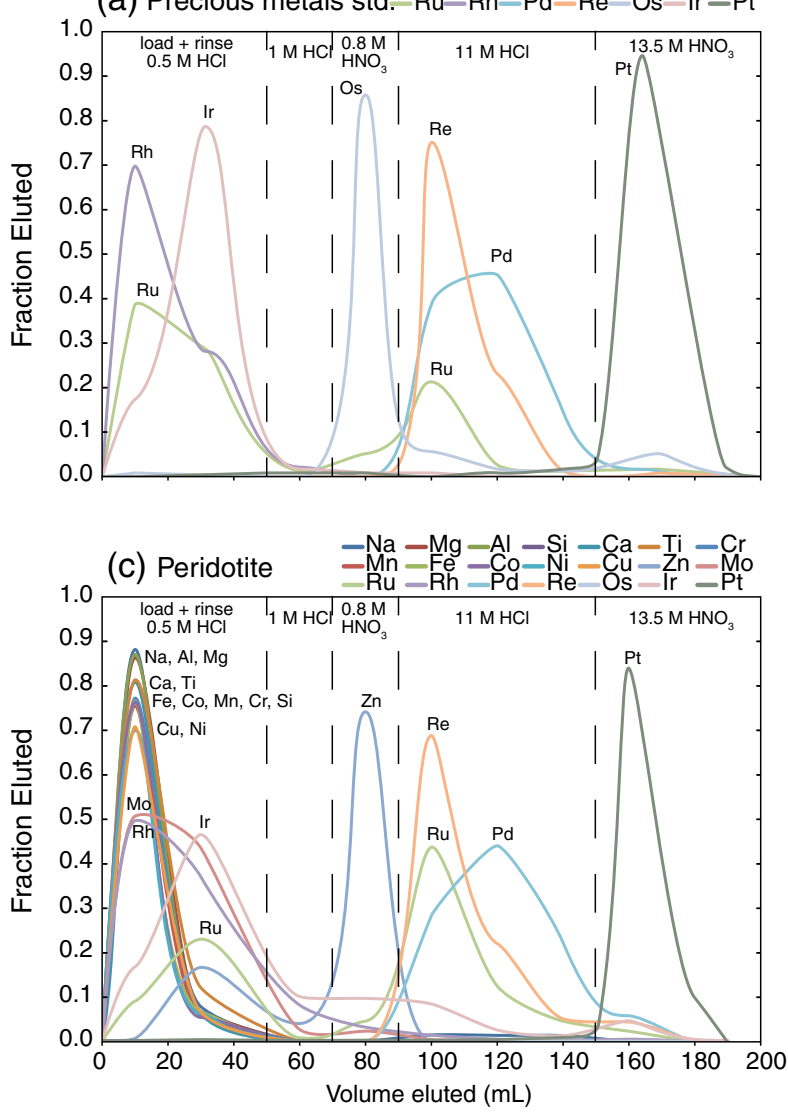
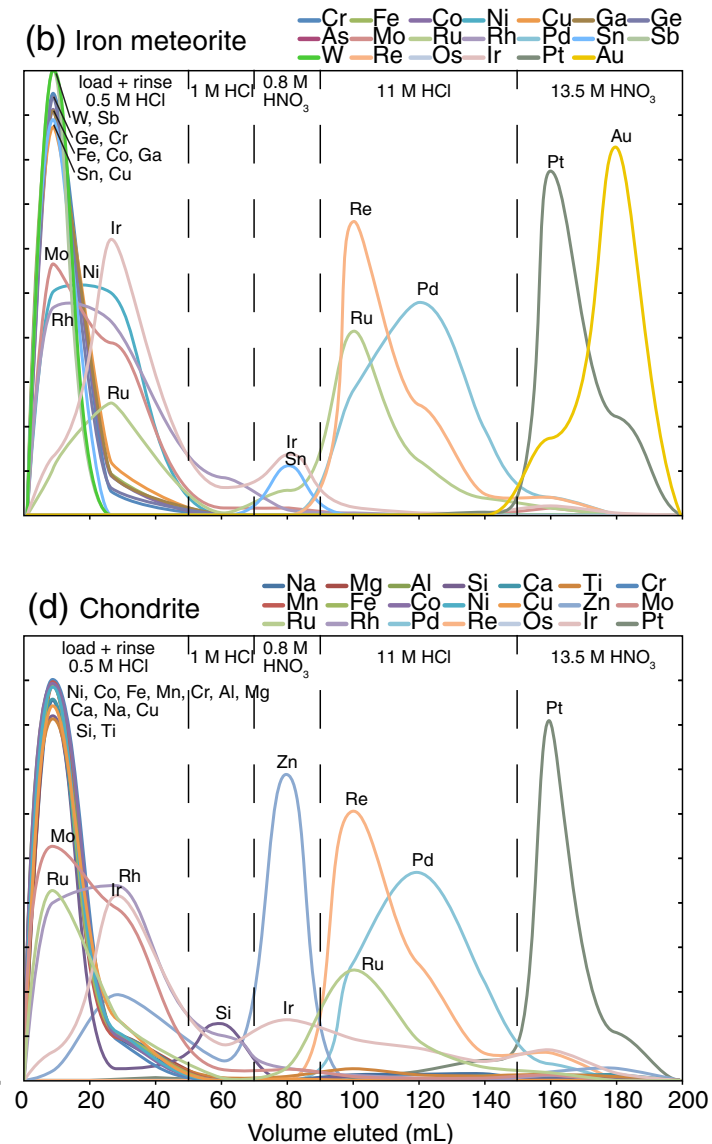

Fig. 2. Elution profiles for (a) precious metal standard solution, (b) synthetic iron meteorite, (c) synthetic peridotite, and (d) synthetic chondrite. In the synthetic rock solutions, the major cationic components are not retained on the column and are eluted with the sample loading solution. The PGE are eluted in a sequence of $\mathrm{HCl}_{\text {and }} \mathrm{HNO} \mathrm{O}_{3}$ acids, and $90-95 \%$ of the $\mathrm{Pt}$ is collected in the $13.5 \mathrm{M} \mathrm{HNO}_{3}$ cut.

with ca. $30 \%$ of the Pd being eluted in the first and last $20 \mathrm{~mL}$ volumes of $11 \mathrm{M} \mathrm{HCl}$, and ca. $40 \%$ in the middle $20 \mathrm{~mL}$ volume of $11 \mathrm{M} \mathrm{HCl}$.

Pt is finally eluted in $40 \mathrm{~mL}$ of $13.5 \mathrm{M} \mathrm{HNO}_{3}$. At least $80 \%$ of Pt is eluted in the first $20 \mathrm{~mL}$ of $13.5 \mathrm{M} \mathrm{HNO}_{3}$, but a further $20 \mathrm{~mL}$ of $13.5 \mathrm{M} \mathrm{HNO}_{3}$ was eluted to ensure (near) complete recovery of Pt. At least $90-95 \%$ of Pt is recovered in the combined $40 \mathrm{~mL}$ of $13.5 \mathrm{M}$ $\mathrm{HNO}_{3}$. This level of recovery was deemed adequate given that isotopic samples were double-spiked prior to anion-exchange chemistry and, thus, incomplete recovery should not affect the measured isotopic compositions of samples. This was tested by processing a sample of IRMM010 through the entire sample preparation procedure, whereby a NiS blank charge was prepared as described above and a double-spiked aliquot of IRMM-010 was added to it. The results of this test are discussed below. Minor amounts of the other PGE were also recovered in the Pt cut, but these were all $<5 \%$ of the Pt concentration, and this level has been previously shown to be sufficient for accurate stable isotope determinations of Pt (Creech et al., 2013).

As a large fraction of Au was collected with Pt, and the effects of this on DS MC-ICPMS isotopic analysis of Pt were only explored up to $10 \%$ of the Pt concentration by Creech et al. (2013), an analytical test was carried out by doping IRMM- 010 with Au at $50 \%$ of the Pt concentration. Au doping at $30 \mathrm{ng} \mathrm{mL}^{-1}$ produced a signal of ca. $3 \mathrm{~V}$ on ${ }^{197} \mathrm{Au}$, which is higher than was observed in any samples analysed in this study. Au doped Pt stable isotope analyses had a reproducibility on $\delta^{198} \mathrm{Pt}$ identical to those of undoped IRMM-010 (ca. $\pm 0.042 ; 2$ sd) and Pt stable isotope ratios identical to pure IRMM-010 (Table 2).

The total procedural blank of the analytical procedure was monitored by processing a blank with each batch of samples through anion-exchange chemistry, both with and without NiS fire assay. Anion-exchange column Pt blanks were typically ca. $\leq 0.1 \mathrm{ng}$. Combined
Pt blanks from NiS digestions and anion-exchange chemistry were typically ca. 0.5-2 ng. The importance of blanks at these levels for accurate isotopic measurements is discussed below.

\subsection{Pt concentrations of standards}

Pt concentrations of the standard reference materials were determined by performing isotope dilution calculations on double-spiked samples, treating ${ }^{198} \mathrm{Pt}$ as the spike isotope and ${ }^{194} \mathrm{Pt}$ as the unspiked isotope (although some ${ }^{194} \mathrm{Pt}$ is also present in the DS). The accuracy of the calculated sample concentrations potentially depends on when spiking was carried out (i.e., prior to or after NiS fire assay digestion) as spiking after digestion will only yield accurate concentrations if the NiS fire assay quantitatively recovers all the Pt. In samples that were double-spiked prior to NiS fire assay, concentrations determined by isotope dilution calculations were between 80 and $108 \%$ of reference Pt concentrations (Table 2). The sample of the komatiite standard OKUM that was double-spiked prior to NiS fire assay had a Pt concentration of $12.4 \mu \mathrm{g} \mathrm{g}^{-1}$ (reference Pt $=11.44 \mu \mathrm{g} \mathrm{g}^{-1}$ ), compared with $9.0 \mu \mathrm{g} \mathrm{g}^{-1}$ when spiking took place after digestion. This is consistent with Pt concentrations determined from replicate WPR-1 digestions, where double-spiking took place after digestion, for which calculated Pt concentrations vary between ca. $80-100 \%$ of the reference value.

\subsection{Platinum stable isotope results}

\subsubsection{Reproducibility of processed standards}

The reproducibility of Pt stable isotopic measurements on the geological standards was estimated by measuring replicate 
Table 2

Pt stable isotope compositions of terrestrial standard reference materials. Uncertainties given for individual digestions represent the weighted means of the number of analyses (n) of each digestion. Uncertainties for replicate digestions are given as the $2 \mathrm{sd}$ of the mean of all digestions. An additional table including all of the individual analyses is available in the Supplementary information.

\begin{tabular}{|c|c|c|c|c|c|c|c|c|c|}
\hline \multirow[b]{2}{*}{ Group } & \multirow[b]{2}{*}{ Sample } & \multirow[b]{2}{*}{ Replicate } & \multirow[b]{2}{*}{$\delta^{198} \mathrm{Pt}$} & \multirow[b]{2}{*}{$\mathrm{n}$} & \multirow{2}{*}{$\frac{\text { Sample Pt }}{(\mathrm{ng})}$} & \multirow{2}{*}{$\frac{\text { Pt conc. }}{\left(\mathrm{ng} \mathrm{g}^{-1}\right)}$} & \multirow{2}{*}{$\frac{\text { Ref. Pt conc. }}{\left(\mathrm{ng} \mathrm{g}^{-1}\right)}$} & \multirow[t]{2}{*}{ Meas./ref. conc. } & \multirow[t]{2}{*}{ DS before digestion? } \\
\hline & & & & & & & & & \\
\hline \multirow[t]{2}{*}{ Anal. tests } & IRMM-010 Au Doped & & $-0.003 \pm 0.042$ & 3 & & & & & \\
\hline & IRMM-010 NiS proc. & & $-0.039 \pm 0.060$ & 7 & & & & & \\
\hline \multirow[t]{20}{*}{ Ore } & PTA-1 & 1 & $0.067 \pm 0.028$ & 6 & & & & & $\mathrm{~N}$ \\
\hline & & 2 & $0.046 \pm 0.015$ & 9 & 2720 & 2719 & 3050 & 0.89 & $\mathrm{Y}$ \\
\hline & & Average & $0.057 \pm 0.030$ & 15 & & & & & \\
\hline & WPR-1 & & & & & & & & \\
\hline & DS after chem. & 1 & $-0.067 \pm 0.016$ & 6 & 417 & 239 & 285 & 0.84 & $\mathrm{~N}$ \\
\hline & & 2 & $-0.107 \pm 0.013$ & 6 & 439 & 251 & 285 & 0.88 & $\mathrm{~N}$ \\
\hline & & 5 & $-0.100 \pm 0.019$ & 4 & 384 & 218 & 285 & 0.77 & $\mathrm{~N}$ \\
\hline & & 6 & $-0.130 \pm 0.016$ & 4 & 420 & 239 & 285 & 0.84 & $\mathrm{~N}$ \\
\hline & & 7 & $-0.087 \pm 0.020$ & 4 & 432 & 246 & 285 & 0.86 & $\mathrm{~N}$ \\
\hline & & average & $-0.098 \pm 0.047$ & 24 & & 240 & 285 & 0.84 & \\
\hline & DS before chem. & 1 & $-0.075 \pm 0.025$ & 4 & 462 & 264 & 285 & 0.93 & $\mathrm{~N}$ \\
\hline & & 2 & $-0.086 \pm 0.026$ & 4 & 489 & 278 & 285 & 0.98 & $\mathrm{~N}$ \\
\hline & & 3 & $-0.116 \pm 0.023$ & 4 & 448 & 255 & 285 & 0.89 & $\mathrm{~N}$ \\
\hline & & 4 & $-0.025 \pm 0.030$ & 3 & 422 & 242 & 285 & 0.85 & $\mathrm{~N}$ \\
\hline & & 5 & $-0.028 \pm 0.035$ & 2 & 403 & 230 & 285 & 0.81 & $\mathrm{~N}$ \\
\hline & & 6 & $-0.036 \pm 0.028$ & 3 & 458 & 262 & 285 & 0.92 & $\mathrm{~N}$ \\
\hline & & 7 & $-0.065 \pm 0.029$ & 3 & 483 & 276 & 285 & 0.97 & $\mathrm{~N}$ \\
\hline & & Average & $-0.061 \pm 0.067$ & 23 & & 258 & 285 & 0.91 & \\
\hline & Overall average & & $-0.077 \pm 0.069$ & 48 & & & & & \\
\hline & SARM-76 & & $-0.052 \pm 0.013$ & 9 & 3955 & 3868 & 3590 & 1.08 & $\mathrm{Y}$ \\
\hline \multirow[t]{6}{*}{ Mantle } & OKUM & 1 & $-0.146 \pm 0.030$ & 3 & 157 & 10.4 & 11.44 & 0.91 & $\mathrm{Y}$ \\
\hline & & 2 & $-0.228 \pm 0.034$ & 2 & 149 & 9.8 & 11.44 & 0.86 & $\mathrm{Y}$ \\
\hline & & 3 & $-0.159 \pm 0.054$ & 2 & 154 & 10.2 & 11.44 & 0.89 & $\mathrm{Y}$ \\
\hline & & Average & $-0.178 \pm 0.088$ & 7 & & 10.1 & 11.44 & 0.89 & \\
\hline & JP-1 & & $-0.335 \pm 0.037$ & 2 & 77 & 5.1 & 5 & 1.02 & $\mathrm{Y}$ \\
\hline & PCC-1 & & $-0.324 \pm 0.053$ & 2 & 96 & 7.9 & 8 & 0.99 & $\mathrm{Y}$ \\
\hline \multirow[t]{5}{*}{ Igneous } & SARM-6 & 1 & $-0.144 \pm 0.017$ & 6 & 588 & 39.2 & 39 & 1.00 & $\mathrm{Y}$ \\
\hline & & 2 & $-0.133 \pm 0.017$ & 6 & 551 & 36.2 & 39 & 0.93 & $\mathrm{Y}$ \\
\hline & & Average & $-0.139 \pm 0.016$ & 12 & & 37.7 & 39 & 0.97 & \\
\hline & DNC-1 & & $-0.050 \pm 0.018$ & 5 & 534 & 35.6 & 36.8 & 0.97 & Y \\
\hline & LK-NIP & & $-0.215 \pm 0.028$ & 2 & 186 & 12.3 & 13.43 & 0.92 & $\mathrm{Y}$ \\
\hline Other & FC-1 & & $-0.020 \pm 0.043$ & 2 & 120 & 30.4 & 38 & 0.80 & $\mathrm{Y}$ \\
\hline
\end{tabular}

a Indicates whether sample was double-spiked prior to sample digestion by NiS fire assay.

digestions of several standard reference materials. Seven replicate digestions of WPR-1 were split and processed twice, once with double-spiking prior to anion-exchange chemistry, and once with double-spiking after anion-exchange chemistry. In addition, the three standards OKUM, SARM- 6 and PTA- 1 were also digested 2-3 times. One digestion of PTA- 1 was double-spiked before NiS digestion and the other was double-spiked prior to anion-exchange chemistry. All digestions of OKUM and SARM-6 were doublespiked prior to NiS digestion. Each processed digestion was typically analysed 3-6 times. Pt stable isotope results for the replicate digestions of standard reference materials are shown in Table 2 and Fig. 3.

Repeat digestions of PTA- 1 and SARM- 6 yielded uncertainties of \pm 0.030 and \pm 0.016 ( 2 sd of weighted means), respectively. Replicates of OKUM were less reproducible, with an uncertainty of \pm 0.088 ( $2 \mathrm{sd}$ of weighted means). WPR- 1 replicates where doublespiking was carried out after anion exchange chemistry yielded a mean $\delta^{198} \mathrm{Pt}=-0.098 \pm 0.047$ ( 2 sd of weighted means), whereas those spiked prior to anion exchange chemistry yielded a mean $\delta^{198} \mathrm{Pt}=-0.061 \pm 0.067$ ( $2 \mathrm{sd}$ of weighted means). These yield a combined average for WPR- 1 of $\delta^{198} \mathrm{Pt}=-0.077 \pm 0.069(2 \mathrm{sd})$. Additionally, the NiS-processed IRMM-010 sample (described in Section 4.1) yielded a mean $\delta^{198} \mathrm{Pt}$ of $-0.039 \pm 0.060$ ( 2 sd; Table 2 ; Fig. 4). The uncertainty demonstrated by these tests must be taken into account when interpreting data from single digestions of samples. Possible explanations for the poorer reproducibility of measurements of

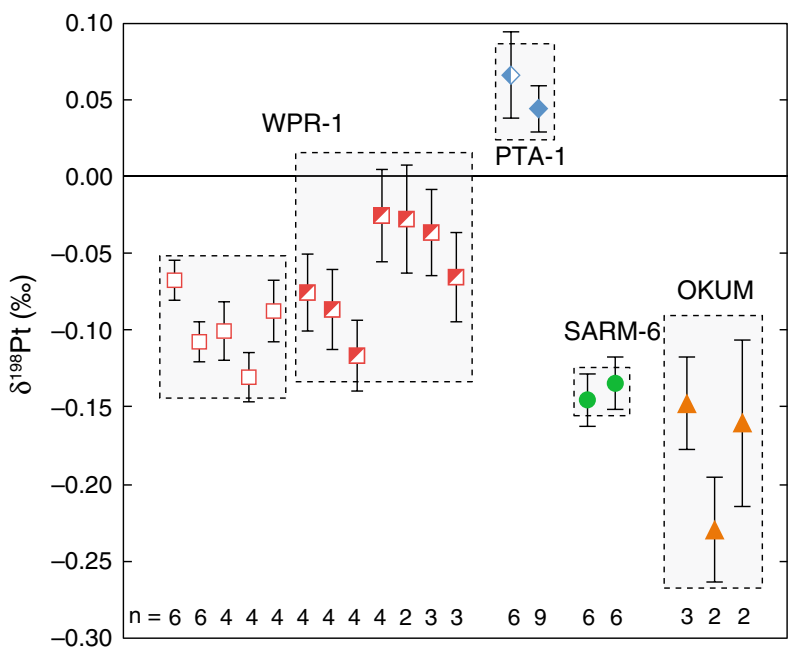

Fig. 3. Pt stable isotopic measurements of the standard reference materials WPR-1, PTA-1, SARM-6 and OKUM, for which replicate digestions were processed. Hollow symbols indicate that the sample was double-spiked after NiS digestion and anion-exchange chemistry, half-filled symbols indicate that the sample was double-spiked after NiS digestion but prior to anion-exchange chemistry, and filled symbols indicate that the sample was double-spiked prior to both NiS digestion and anion-exchange chemistry. Data points are error-weighted means of the number of analyses ( $\mathrm{n}$ ) indicated below each data point, with associated combined errors. Dashed lines indicate the 2 sd uncertainty from all of the replicates they enclose. 
$\delta^{198} \mathrm{Pt}(\%)$

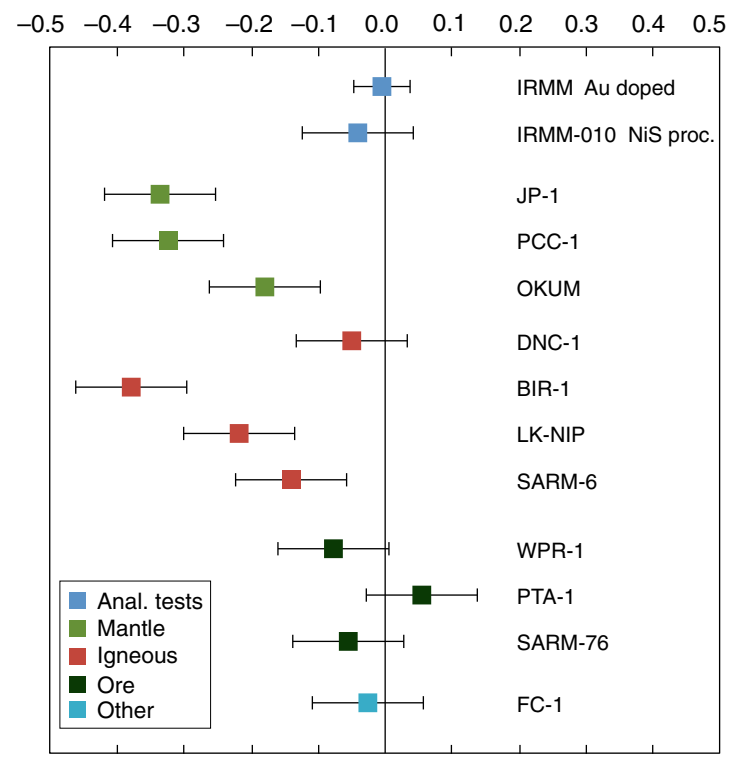

Fig. 4. Pt stable isotope variations in standard reference materials relative to IRMM-010. Error bars reflect the reproducibility as determined by the $2 \mathrm{sd}$ of replicate digestions of OKUM of \pm 0.088 (Table 2).

replicate digestions of WPR-1 and OKUM compared with that of Pt solution standards include:

a) Anion exchange chemistry yields. WPR-1 replicates spiked before and after chemistry were spiked with identical amounts of DS. Isotope dilution concentration results for WPR-1 replicates spiked after anion exchange chemistry have ca. 10\% lower apparent Pt concentrations than those spiked prior to chemistry, consistent with elution results shown in Section 4.1. The overlap in $\delta^{198} \mathrm{Pt}$ results for replicates spiked before and after anion exchange chemistry suggests that incomplete recovery of Pt during anion-exchange chemistry does not isotopically fractionate Pt significantly at this level of recovery. Additionally, no correlation is observed between $\delta^{198} \mathrm{Pt}$ and Pt recovery within WPR-1 replicates spiked after chemistry (Table 2). Thus, yields from anion exchange chemistry cannot explain the variations observed in the WPR-1 replicates.

b) Incomplete sample-spike equilibration. Incomplete sample-spike equilibration could result in an apparent isotopic fractionation of a sample. Although all of the WPR-1 replicates were subjected to identical preparation that was intended to equilibrate the sample and DS, incomplete and inconsistent equilibration could explain some of the variability in the WPR-1 replicates, and potentially explain why the replicates double-spiked before anion-exchange chemistry show poorer reproducibility than those double-spiked after chemistry. However, samples double-spiked prior to digestion are considered to have fully equilibrated.

c) NiS fire assay blanks. Pt blanks from the NiS fire assay technique were found to vary between ca. $0.5-2.0 \mathrm{ng}$. The influence of blank Pt is difficult to assess without knowing the isotopic composition of the blank, and depends also on the Pt content of the sample. Given that each digestion of WPR-1 involved processing of $\mathrm{ca}$. $4000 \mathrm{ng}$ of Pt it can be calculated that the stable Pt isotopic composition of the blank would need to have an implausibly high $\delta^{198} \mathrm{Pt}$ of ca. +10 and be highly variable either in terms of this blank composition or content to account for the range of $\delta^{198} \mathrm{Pt}$ values exhibited by the replicate digestions of WPR-1. Furthermore, the Pt concentrations obtained for our most Pt depleted samples agree well with published reference values for these standards. d) Spectral or non-spectral matrix effects from residual organics from anion exchange resin. Element doping tests have shown that minor amounts of the most likely contaminants from incomplete Pt separation should not affect isotopic measurements (Creech et al., 2013). As described above, Pt cuts were treated with aqua regia and $\mathrm{HNO}_{3}-\mathrm{H}_{2} \mathrm{O}_{2}$ to attempt to destroy any organics stripped from the anion exchange resin. While this is considered to be effective, the presence of any residual organics may have variable spectral or non-spectral matrix effects that could explain the variations observed between replicates of WPR-1.

e) True isotopic heterogeneity of the sample. This may be more apparent with OKUM than the other standards due to the lower Pt concentration in that standard. However, due to its lower concentration, each replicate of OKUM was only measured 2-3 times, which also affects the resulting uncertainty. Further work is required to establish the level of Pt isotopic heterogeneity within various types of samples. It is hoped that additional investigations utilising these methods will be forthcoming, which will help to resolve this question.

The relatively consistent results between replicate digestions of SARM- 6 and PTA-1 suggest that reproducibility may be improved where samples are double-spiked prior to digestion (Fig. 3). However, for the data for natural samples presented in Fig. 4 we have conservatively imposed uncertainties of \pm 0.088 (Table 2), as determined from replicate digestions of OKUM.

\subsubsection{Pt stable isotope variations in terrestrial rock standards}

The 11 standard reference materials analysed in this study showed Pt stable isotope variations that are readily resolvable by this technique (Fig. 4; Table 2). The Pt stable isotope composition of Earth's mantle as defined by mantle samples JP-1, PCC-1 and OKUM, is clearly distinct from those of the ultramafic ore deposits PTA-1 and SARM-76. The two peridotites (JP-1 and PCC-1) have identical Pt stable isotope compositions within analytical uncertainty $\left(\delta^{198} \mathrm{Pt}=-0.335\right.$ and -0.324 , respectively).

The ore materials, WPR-1, SARM-76 and PTA-1, have the heaviest Pt isotopic compositions, which are similar to the composition of the IRMM-010 standard (i.e. close to zero). The similarity between the ore materials and IRMM-010 may be consistent with the IRMM-010 Pt isotope standard having been ultimately derived from a Pt ore. The igneous group of reference materials generally have Pt stable isotopic compositions intermediate between the mantle and ores. Although the ores used in this study may have formed through different processes, the isotopic offset between igneous samples and ores suggests that there may an isotopic fractionation associated with the removal of Pt from silicate magmas to form Pt ore deposits. The Icelandic basalt BIR-1, which is a mafic and isotopically depleted product of the Iceland plume, has the most extreme Pt stable isotopic composition of $\delta^{198} \mathrm{Pt}=-0.377 \pm 0.051$. This composition is similar to that of the peridotite samples, which are considered to represent the upper mantle.

DNC-1 has the heaviest Pt stable isotopic composition of the igneous standard reference materials $\left(\delta^{198} \mathrm{Pt}=-0.050\right)$. DNC- 1 is also characterised by an unusually high Pt concentration (38 $\mathrm{ng} \mathrm{g}^{-1}$; Table 1) compared with other igneous samples. The isotopic similarity between DNC-1 and the ore samples coupled with its relative enrichment in Pt potentially indicates that either the source deposit of DNC1 is associated with sulphide-enriched ultramafic deposits or that the DNC-1 standard was contaminated during crushing and preparation of the standard. Similar contamination during preparation and crushing of geological standard reference materials has been documented for other isotopic systems (e.g., Pb; Baker et al., 2004).

The reproducibility of Pt stable isotope data documented in the standard reference materials of which multiple digestions were made in this study, particularly SARM-6 and PTA-1, demonstrates that these could be 
potentially useful as isotopic standards in future applications of Pt and other highly siderophile element stable isotope studies.

\section{Conclusions}

We have developed a chemical separation procedure for purification of Pt from geological and potentially from meteoritic samples for $\mathrm{Pt}$ stable isotopic analysis. We have shown:

1. Pt can be separated from various rock matrices with a recovery of 90-95\% and with sufficient purity (>95\%) for accurate Pt stable isotope determinations by double-spike MC-ICPMS.

2. While the current reproducibility of processed replicates of terrestrial rock standards (e.g., ca. \pm 0.088 for OKUM) is poorer than for repeat measurements of Pt solution standards (ca. \pm 0.040 ), this level of precision appears to be adequate to resolve variations between natural samples.

3. Pt stable isotopic analyses of 11 terrestrial standard reference materials show a readily resolvable range in $\delta^{198} \mathrm{Pt}$, with offsets of up to ca. 0.4 for some standards

These results demonstrate that the Pt stable isotope system holds considerable promise as a tracer in geochemical systems.

Supplementary data to this article can be found online at http://dx. doi.org/10.1016/j.chemgeo.2013.11.009.

\section{Acknowledgements}

The authors thank two anonymous reviewers for their insightful comments and Laurie Reisberg for her editorial efforts, which improved the manuscript. This research was supported by a Royal Society of New Zealand Marsden Grant to MH and JB. JC is funded by a VUW $\mathrm{PhD}$ scholarship. The Centre for Star and Planet Formation is funded by the Danish National Research Foundation and the University of Copenhagen's Programme of Excellence.

\section{References}

Alvarez, L.W., Alvarez, W., Asaro, F., Michel, H.V., 1980. Extraterrestrial cause for the Cretaceous-Tertiary extinction. Science 208, 1095-1108. http://dx.doi.org/10.1126/ science.208.4448.1095.

Baker, J., Peate, D., Waight, T., Meyzen, C., 2004. Pb isotopic analysis of standards and samples using a ${ }^{207} \mathrm{~Pb}-{ }^{204} \mathrm{~Pb}$ double spike and thallium to correct for mass bias with a double-focusing MC-ICP-MS. Chem. Geol. 211, 275-303. http://dx.doi.org/10.1016/ j.chemgeo.2004.06.030

Barkov, A.Y., Laflamme, J.H.G., Cabri, L.J., Martin, R.F., 2002. Platinum-group minerals from the Wellgreen Ni-Cu-PGE deposit, Yukon, Canada. Can. Mineral. 40, 651-669. http://dx.doi.org/10.2113/gscanmin.40.2.651.

Cotton, F.A., Wilkinson, G., 1966. Advanced Inorganic Chemistry: A Comprehensive Text. Interscience Publishers.

Creech, J., Baker, J., Handler, M., Schiller, M., Bizzarro, M., 2013. Platinum stable isotope ratio measurements by double-spike multiple collector ICPMS. J. Anal. At. Spectrom. 28, 853-865. http://dx.doi.org/10.1039/C3JA50022E.

Creech, J., Paul, B., 2013. IsoSpike: improved double-spike deconvolution software. Geostand. Geoanal. Res. (submitted for publication).
Faye, G.H., Sutarno, R., 1976. Certified compositional reference materials for the earth sciences. Can. Mineral. 14, 164-171.

Findlay, D.C., 1969. Origin of the Tulameen ultramafic-gabbro complex, southern British Columbia. Can. J. Earth Sci. 6, 399-425. http://dx.doi.org/10.1139/e69-040.

Gros, M., Lorand, J., Luguet, A., 2002. Analysis of platinum group elements and gold in geological materials using NiS fire assay and Te coprecipitation; the NiS dissolution step revisited. Chem. Geol. 185, 179-190.

Hart, T.R., MacDonald, C.A., 2007. Proterozoic and Archean geology of the Nipigon Embayment: implications for emplacement of the Mesoproterozoic Nipigon diabase sills and mafic to ultramafic intrusions. Can. J. Earth Sci. 44, 1021-1040. http://dx.doi.org/ 10.1139/E07-026

Heaman, L., Easton, R., Hart, T., Hollings, P., MacDonald, C., Smyk, M., 2007. Further refinement to the timing of Mesoproterozoic magmatism, Lake Nipigon region, Ontario. Can. J. Earth Sci. 44, 1055-1086. http://dx.doi.org/10.1139/E06-117.

Houlé, M.G., Préfontaine, S., Fowler, A.D., Gibson, H.L., 2009. Endogenous growth in channelized komatiite lava flows: evidence from spinifex-textured sills at Pyke Hill and Serpentine Mountain, Western Abitibi Greenstone Belt, Northeastern Ontario, Canada. Bull. Volcanol. 71, 881-901. http://dx.doi.org/10.1007/s00445-009-0273-y.

Marcantonio, F., Reisberg, L., Zindler, A., Wyman, D., Hulbert, L., 1994. An isotopic study of the Ni-Cu-PGE-rich Wellgreen intrusion of the Wrangellia Terrane: evidence for hydrothermal mobilization of rhenium and osmium. Geochim. Cosmochim. Acta 58 1007-1018. http://dx.doi.org/10.1016/0016-7037(94)90522-3.

Meisel, T., Moser, J., 2004. Reference materials for geochemical PGE analysis: new analytical data for Ru, Rh, Pd, Os, Ir, Pt and Re by isotope dilution ICP-MS in 11 geological reference materials. Chem Geol. 208, 319-338. http://dx doi.org/10.1016/j.chemgeo.2004.04.019.

Morcelli, C.P.R., Figueiredo, A.M.G., Enzweiler, J., Sarkis, J.E.S., Jorge, A.P.S., Kakazu, M., 2004. Determination of Platinum-Group elements in geological reference materials by high resolution-ICP-MS after nickel sulfide fire-assay collection and Te co-precipitation. Geostand. Geoanal. Res. 28, 305-310. http://dx.doi.org/10.1111/j.1751908X.2004.tb00745.X.

Nisbet, E.G., Walker, D., 1982. Komatiites and the structure of the Archaean mantle. Earth Planet. Sci. Lett. 60, 105-113. http://dx.doi.org/10.1016/0012-821X(82)90025-5.

Paton, C., Hellstrom, J., Paul, B., Woodhead, J., Hergt, J., 2011. Iolite: freeware for the visualisation and processing of mass spectrometric data. J. Anal. At. Spectrom. 26 2508-2518. http://dx.doi.org/10.1039/c1ja10172b.

Pearson, D.G., Woodland, S.J., 2000. Solvent extraction/anion exchange separation and determination of PGEs (Os, Ir, Pt, Pd, Ru) and Re-Os isotopes in geological samples by isotope dilution ICP-MS. Chem. Geol. 165, 87-107. http://dx.doi.org/10.1016/S00092541(99)00161-8.

Ravizza, G., Pyle, D., 1997. PGE and Os isotopic analyses of single sample aliquots with NiS fire assay preconcentration. Chem. Geol. 141, 251-268.

Rehkämper, M., Halliday, A.N., 1997. Development and application of new ion-exchange techniques for the separation of the platinum group and other siderophile elements from geological samples. Talanta 44, 663-672. http://dx.doi.org/10.1016/S00399140(96)02100-5.

Rudge, J.F., Reynolds, B.C., Bourdon, B., 2009. The double spike toolbox. Chem. Geol. 265 420-431. http://dx.doi.org/10.1016/j.chemgeo.2009.05.010.

Savard, D., Barnes, S., Meisel, T., 2010. Comparison between nickel-sulfur fire assay Te coprecipitation and isotope dilution with high-pressure asher acid digestion for the determination of platinum-group elements, rhenium and gold. Geostand. Geoanal. Res. 34, 281-291. http://dx.doi.org/10.1111/j.1751-908X.2010.00090.x.

Takazawa, E., Frey, F., Shimizu, N., Obata, M., 1996. Evolution of the Horoman Peridotite (Hokkaido, Japan): implications from pyroxene compositions. Chem. Geol. 134, 3-26. http://dx.doi.org/10.1016/S0009-2541(96)00083-6.

Takazawa, E., Frey, F., Shimizu, N., Obata, M., 2000. Whole rock compositional variations in an upper mantle peridotite (Horoman, Hokkaido, Japan): are they consistent with a partial melting process? Geochim. Cosmochim. Acta 64, 695-716. http://dx.doi.org/ 10.1016/S0016-7037(99)00346-4.

Takazawa, E., Frey, F.A., Shimizu, N., Obata, M., Bodinier, J.L., 1992. Geochemical evidence for melt migration and reaction in the upper mantle. Nature 359, 55-58. http:// dx.doi.org/10.1038/359055a0.

Takazawa, E., Frey, F.A., Shimizu, N., Saal, A., Obata, M., 1999. Polybaric petrogenesis of mafic layers in the Horoman Peridotite Complex, Japan. J. Petrol. 40, 1827-1851. http://dx.doi.org/10.1093/petroj/40.12.1827.

Walraven, F., Armstrong, R., Kruger, F., 1990. A chronostratigraphic framework for the north-central Kaapvaal craton, the Bushveld Complex and the Vredefort structure. Tectonophysics 171, 23-48. http://dx.doi.org/10.1016/0040-1951(90)90088-P. 


\section{Appendix D}

\section{Preprint of $G G R$ paper (IsoSpike)}

Several chapters in this thesis refer to a tool that was developed for reduction of double-spike mass spectrometry data by Creech and Paul (2014). This tool is now called IsoSpike, and is described in an article that is, at the time of writing, in press in Geostandards and Geoanalytical Research. This appendix includes a preprint version of the article, which is entitled IsoSpike: Improved Double-Spike Deconvolution Software. This pre-print version has not yet been formatted to the final style of the journal. The final version will be available online using the DOI: 10.1111/j.1751-908X.2014.00276.x. 
Received Date: 20-Jul-2013

Revised Date: 26-Nov-2013

Accepted Date: 09-Dec-2013

Article Type: Original Article

IsoSpike: Improved Double-Spike Inversion Software

John B. Creech $(1)^{*}$ and Bence Paul $(2,3)$

(1) School of Geography, Environment and Earth Sciences, Victoria University of Wellington, PO Box 600, Wellington 6140, New Zealand

(2) School of Earth Sciences, The University of Melbourne, Parkville, VIC, Australia

(3) CODES ARC Centre of Excellence in Ore Deposits and School of Earth Sciences, University of Tasmania, Tasmania, Australia

*Corresponding author. e-mail: john.creech@vuw.ac.nz

The double-spike approach for instrumental mass bias correction of mass spectrometry data is well established. However, there is very little consistency within the scientific community in terms of double-spike data reduction. Double-spike solutions require computer calculation, using either geometric or algebraic approaches, and are often performed using spreadsheet calculations that vary from group to group and between isotope systems. Here we present IsoSpike, a generalised computer procedure for the processing of double-spike mass spectrometry data, built as an add-on for the lolite data reduction package (www.iolite.org.au). Use of this software permits visualisation of mass spectrometry data in a time window, and rigorous treatment and screening of data. Additionally, IsoSpike uses an integration-byintegration approach where the double-spike calculations are performed on every integration within an analysis, providing straightforward quantification of internal errors on double spikecorrected isotope ratios. The advantages of this approach over traditional methods are This article has been accepted for publication and undergone full peer review but has not been through the copyediting, typesetting, pagination and proofreading process, which may lead to differences between this version and the Version of Record. Please cite this article as doi: 10.1111/j.1751-908X.2014.00276.x

This article is protected by copyright. All rights reserved. 


\section{discussed here. Platinum stable isotope data are presented as an example data set, although the procedure is applicable to any double-spike system. IsoSpike is freely available from www.isospike.org.}

Keywords: double-spike, isotopes, MC-ICP-MS, TIMS, software.

\section{Received 20 Jul 13 - Accepted 09 Dec 13}

Isotope geochemistry has played a vital part in the study of Earth. Radiometric dating has provided information on the age of the Earth, while radiogenic and stable isotopic tracers have been used to delineate processes occurring in the mantle and crust as well as the ocean and atmosphere. One of the limiting factors in the accuracy of isotope geochemistry, however, is the effect of instrumental mass bias. Instrumental mass bias is an analytical effect whereby high atomic number or low atomic number ions may be preferentially detected for different reasons depending on the instrumentation, potentially occurring during evaporation, ionisation or transmission. Instrumental mass bias occurs in both inductively coupled plasma-mass spectrometry (ICP-MS) and thermal ionisation mass spectrometry (TIMS), but in an opposite sense in that ions of higher mass are preferentially detected in ICP-MS as compared with TIMS. Although there are a variety of means to correct for mass bias, all are empirical, and it is commonly accepted that the double-spike (DS) method (Dodson 1963) is the most precise and accurate where no invariant pair of isotopes are available for mass bias correction or the stable isotopic composition of the sample is being measured (Woodhead and Hergt 1997, Anbar 2004). The advantages of the DS approach have been known since the 1960s (e.g., Compston and Oversby 1969) although at the time, calculations for a single result took more than $15 \mathrm{~min}$ (Dodson 1970) and the equations to describe mass bias were typically linear. In the intervening period, more complex equations have been developed to describe mass bias effects (for a summary, see Maréchal et al. 1999) and the use of computers has become essential.

While virtually all laboratories producing DS data employ the same algebraic or geometric approaches to the DS correction, there is very little consistency in their implementation. DS data reduction is often performed in complicated spreadsheets, which vary between isotope systems and groups. We aim to provide a generalised, easy to use and freely available tool that is applicable to any double-spike system, and which will provide greater consistency in the data reduction process. We present IsoSpike, a software tool that can perform many thousands of double-spike inversions per second on a modern personal computer, allowing the analyst to

This article is protected by copyright. All rights reserved. 
calculate mass bias corrected ratios on an integration-by-integration basis within an analysis. This approach also provides a straightforward method for quantification of measurement repeatability, which have previously been estimated by linear uncertainty propagation (Rudge et al. 2009), Monte Carlo simulation (Fantle and Bullen 2009, John 2012) or by a least-squares approach (Powell et al. 1998). We provide examples of data reduction using this approach with the platinum stable isotope system.

\section{The double-spike technique}

Various methods are available for the correction of instrumental mass bias in isotope measurements. Reference material (RM) bracketing is one of the most commonly used, whereby analyses of unknowns are bracketed with measurements of a reference material (calibrator) of known composition, and the difference between the measured and reference ratios for the RM are used to determine the instrumental mass bias. The disadvantage of this method is that it requires many additional analyses of RMs to be made, which is time consuming, and often assumes that changes in mass bias are linear between bracketing calibrators. For some radiogenic isotope systems mass bias can be determined within the analysis of the unknown by assuming that some ratio is invariant in nature (e.g., two stable non-radiogenic isotopes). Alternatively, in some cases a sample may be doped with another element that exhibits similar mass bias behaviour and has a well constrained isotope ratio (e.g., $\mathrm{Tl}$ in $\mathrm{Pb}$ isotope work; Woodhead 2002, Baker et al. 2004), which is then be used to perform the same correction.

The double-spike technique is a variation on internal correction methods, whereby the presence of an invariant ratio is simulated by doping the sample with a solution that is enriched in two isotopes of the element in a known ratio, the so-called double-spike. The fundamentals of the DS approach have been well documented (Dodson 1963, 1970, Compston and Oversby 1969, Hamelin et al. 1985, Powell et al. 1998, Galer 1999, Johnson and Beard 1999, Rudge et al. 2009), and the technique has been used extensively in a range of isotopic systems - most notably $\mathrm{Pb}$, but also S, Ca, Cr, Zn, Fe, Mo, Cd, Ba and Os, and using both ICP-MS and TIMS. In practice it is impossible to produce a $100 \%$ pure DS (i.e., the spike will include both the enriched isotopes and a contribution from the naturally occurring isotopes), and the relative contributions of the spike and sample in the mixture must be taken into account. Mathematical solutions to this problem have been developed, and the sample contribution can be resolved from the spike contribution. With knowledge of the composition of the double-spike, it is therefore possible to

This article is protected by copyright. All rights reserved. 
calculate the instrumental mass bias from mass spectrometry data, and thus derive the natural fractionation of the sample.

The double-spike equations used here are derived by combining exponential mass bias laws describing the instrumental and natural isotopic fractionation, and a mixing relationship between the double-spike and the sample (a thorough treatment of the mathematics is provided in Rudge et al. 2009). The result is the following set of non-linear simultaneous equations:

$F_{i}(\lambda, \alpha, \beta, n, m, T)=\lambda T_{i}+(1-\lambda) n_{i} e^{-\alpha P_{i}}-m_{i} e^{-\beta P_{i}}=0$

where the subscript $i$ refers to the $i$ th isotopic ratio of the sample; $P$ is the natural logarithm of the ratio of atomic masses; $m$ and $n$ refer to the measured compositions of the sample-spike mixture and unspiked sample, respectively; $T$ represents the composition of the double-spike; $\lambda$ is related to the proportion in which the sample and double-spike are mixed; $\alpha$ and $\beta$ are the natural and instrumental mass fractionation factors, respectively. The double-spike inversion takes the values $n, m$ and $T$ for inputs, which are used for the solution of the equation above for the unknowns $\lambda$, $\alpha$, and $\beta$. Three isotope ratios are required to solve these equations, and thus the double-spike approach is limited to elements with four or more isotopes.

When the double-spike technique was first used, the equations involved were necessarily simplified to a linear form (Compston and Oversby 1969, Dodson 1970). Since then, complex methods for the computation of double-spike corrections have been developed utilising both algebraic (Albarède and Beard 2004, Rudge et al. 2009) and geometric (Russell 1971, Galer 1999, Johnson and Beard 1999, Siebert et al. 2001, Markey et al. 2003) approaches.

The DS method poses several additional advantages over some of the other methods described above. For stable isotope systems where mass-dependent fractionation is being studied, DScorrected isotope ratios can be obtained from a single analysis (Rudge et al. 2009). Additionally, the DS can potentially correct for any mass-dependent effects to the sample after addition of the DS. Thus if the addition of the DS to the sample is performed prior to chemical separation of the element, the DS will also correct for any mass-dependent fractionation such as might occur as a result of incomplete yields during chemical separation (Albarède and Beard 2004, Rudge et al. 2009).

This article is protected by copyright. All rights reserved. 


\section{Software}

The DS procedure presented here is called IsoSpike, and is freely available from www.isospike.org. IsoSpike was built as an add-on module for the non-commercial software package lolite (Paton et al. 2011), which is available from www.iolite.org.au. Iolite is a datareduction package for visualisation and processing of mass spectrometric data, which itself runs within the data analysis package Igor Pro (available from www.wavemetrics.com; see Paton et al. (2011) for further details). Here we provide an example using the platinum isotope system, although IsoSpike is applicable to any isotope system suitable for the DS method. The Iolite platform is capable of directly handling data from a variety of different mass spectrometers using the same data reduction steps, and can reduce large data sets quickly and easily, making it ideal for this purpose.

\section{Data reduction process}

Once data are imported into lolite they are displayed in a window versus time. The user can then manually select integration periods for each sample/reference material-DS mixture or use an automated process within Iolite to do so (Figure 1). A relevant Data Reduction Scheme (DRS) is then selected and run (e.g., the Pt_DS DRS, which has been written for Pt isotopes and is used in the example files). The DRS performs the initial processing steps of baseline subtraction and the calculation of raw isotope ratios, which are then used as inputs for IsoSpike. IsoSpike can be called from within the DRS or run manually to perform the DS calculations.

IsoSpike employs the algebraic method and terminology of Rudge et al. (2009). The required DS parameters are: the measured composition of the sample-DS mixture $(m)$; the measured value of the unspiked natural sample or the composition of a reference material $(n)$; the isotopic composition of the DS (T); and the natural logarithm of the ratio of the isotopic masses (P; Equation 1). These parameters can be input manually or loaded from a saved settings file. In the first instance, a user would typically go through the process of creating a DS settings file (Figure 2). This process is guided by the software, and IsoSpike contains a database of IUPAC recommended isotopic masses (Berglund and Wieser 2011) with an interface for the user to select which isotopes are used in the DS inversion, and the required mass ratios can be calculated automatically. Thus, to change to a new isotope system, the only information that must be input manually is the DS composition and, depending on the application, the composition of a reference material.

This article is protected by copyright. All rights reserved. 
Once all the parameters have been loaded, IsoSpike performs the DS inversion to solve for three unknowns: the sample-spike mixing proportion, $\lambda$, and the fractionation factors $\alpha$ and $\beta$ (Equation 1). Several iterative methods are available for finding roots of non-linear equations; IsoSpike employs the FindRoots function in Igor Pro, which uses the Jenkins-Traub algorithm (Jenkins and Traub 1975). IsoSpike performs these calculations on a integration-by-integration basis, allowing outlier rejection and calculation of statistics to be performed directly on calculated results (Figure 3). This procedure is capable of rapidly reducing a typical analytical session worth of data in a few seconds on a modern personal computer.

The integration-by-integration method employed here is compared with a commonly-used method in which DS calculations are performed on means of the three DS ratios for each analysis, which is hereafter referred to as the means-of-ratios approach. In mean-of-ratios methods, uncertainties cannot be quantified directly but can be estimated by one of several available methods (Monte Carlo simulation, linear error propagation, least squares). By performing the DS calculations on each integration within an analysis, the IsoSpike permits direct quantification of uncertainties. The potential advantages of this approach are discussed below.

\section{Unspiked run}

In general, the double-spike method involves an additional measurement of each sample without the DS added, the so-called 'unspiked run'. In isotope systems where mass-dependent isotopic fractionation is being studied, it is possible to compute DS-corrected isotope ratios from a single, spiked, measurement using the value of an isotopic RM for the natural 'unspiked' composition, and DS-corrected ratios are then expressed relative to that RM. IsoSpike can operate in either of these modes, although the pre-processing in the DRS is slightly different. Where an unspiked run is measured, the method employs a fitted spline in lolite so that the measured value of the unspiked sample exists in the same time interval as the spiked run. Where no unspiked run is required, the standard composition is defined in the DS settings file.

\section{Materials and analytical methods}

The materials and methods for $\mathrm{Pt}$ isotope measurements are described in detail in Creech et al. (2013). A short outline is also provided here.

This article is protected by copyright. All rights reserved. 


\section{Chemicals and standard solutions}

Optima ${ }^{\mathrm{TM}}$ grade nitric $\left(\mathrm{HNO}_{3}\right)$ and hydrochloric $(\mathrm{HCl})$ acids were used as supplied without further purification. Acid dilutions were performed with ultrapure (> $18 \mathrm{M} \Omega \mathrm{cm}$ ) water. All sample handling and preparation was performed in a class-100 ultra-clean laboratory at Victoria University of Wellington, New Zealand.

The Pt isotope reference material IRMM-010 was purchased from the Institute for Reference Materials and Measurements, Belgium (Wolff Briche et al. 2002). The reference material was supplied in the form of Pt wire segments, which were cleaned prior to digestion by sequentially rinsing in ultrapure water, analytical grade methanol and dilute $(5 \% \mathrm{v} / \mathrm{v}) \mathrm{HNO}$. The IRMM-010 material was carefully weighed, and then digested in aqua regia $\left(\mathrm{HCl}: \mathrm{HNO}_{3}\right.$ prepared in a volume ratio of 3:1) in a Savillex ${ }^{\mathrm{TM}}$ Teflon beaker. The IRMM-010 Pt solution was evaporated to dryness, refluxed with concentrated $\mathrm{HNO}_{3}$, evaporated to dryness, and finally redissolved in $20 \%$ $\mathrm{v} / \mathrm{v} \mathrm{HCl}$. Measurements of a Pt ICP-MS in-house reference solution (J.T. Baker $1000 \mathrm{\mu g} \mathrm{ml}^{-1}$ in $20 \%$ $\checkmark / \mathrm{v} \mathrm{HCl}$, Lot\# H44N77) are also included for comparison, which has been shown to have a small but reproducible offset from IRMM-010 (Creech et al. 2013).

The Pt double-spike used for the isotopic determinations was prepared using two single isotope spikes (196Pt and $198 \mathrm{Pt}$ ) obtained from the Oak Ridge National Laboratory, USA. Full details of the design and calibration of the double spike are given in Creech et al. (2013). Solutions of IRMM010 and the reference solution of J.T. Baker were spiked immediately prior to MC-ICP-MS measurement, spiking in optimal proportions as calculated using the Double Spike Toolbox of Rudge et al. (2009).

Analytical methods

Platinum isotope measurements were performed using a Nu Plasma HR MC-ICP-MS (NU Instruments) at Victoria University of Wellington, New Zealand. The MC-ICP-MS was operated in low-resolution mode, with a mass resolution of 1840 (M/AM; peak edge width from 5-95\% full peak height). A Nu Instruments DSN-100 desolvation nebuliser system with an ESI PFA micro-flow nebuliser was used for Pt solution introduction to the mass spectrometer. Measurements were typically made on $0.5 \mathrm{~mol} \mathrm{I}^{-1} \mathrm{HCl}$ solutions with $60 \mathrm{ng} \mathrm{ml}^{-1}$ of Pt from the sample, with an almost equivalent contribution from DS Pt. The sample uptake rate was ca. $80 \mu \mathrm{l} \mathrm{min-1}$, and the sensitivity of the instrument was typically ca. $280 \mathrm{~V}$ per $\mu \mathrm{g} \mathrm{ml} \mathrm{ml}^{-1}$ total Pt under these conditions. Peak centring was performed at the start of each session and monitored throughout. Each individual analysis comprised $100 \times 8 \mathrm{~s}$ integrations, corresponding to $13.3 \mathrm{~min}$ of data acquisition, and

This article is protected by copyright. All rights reserved. 
consumed ca. $80 \mathrm{ng}$ of natural Pt. Baselines were obtained by measuring on-peak for $100 \times 8 \mathrm{~s}$ integrations prior to each analysis. A washout time of ca. 30 min was applied between analyses and baseline measurements. Using this approach, background levels were typically $<<1 \mathrm{mV}$ on all Pt isotopes.

Results are reported using $\delta^{198} \mathrm{Pt}$ notation, representing the per mil deviation of the ${ }^{198} \mathrm{Pt} / 194 \mathrm{Pt}$ ratio from the same ratio for the IRMM-010 Pt isotope RM:

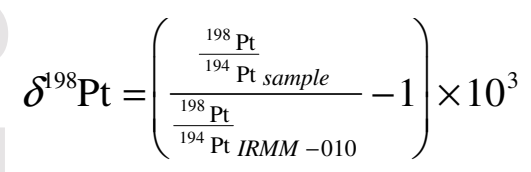

\section{Results and discussion}

The integration-by-integration processing of data within IsoSpike presents several advantages over traditional DS data reduction approaches using means-of-ratios and modelled uncertainty estimates. The differences between IsoSpike and traditional methods are explored below.

\section{DS-corrected ratios}

The integration-by-integration inversion approach used in IsoSpike was compared with data corrected from means-of-ratios from each analysis by performing the DS calculations on the same data using both methods. Means-of-ratios results were calculated using the same procedure in IsoSpike, but instead using the means-of-ratios for each analysis for inputs. Uncertainties on means-of-ratios data were determined using the linear uncertainty propagation routine (ERRORESTIMATE) within the Double Spike Toolbox of Rudge et al. (2009).

The mean DS-corrected $\delta^{198} \mathrm{Pt}$ values for each analysis were generally within ca. $5 \%$ (relative) between the two methods (Figure 4, Table 1). However, the integration-by-integration method resulted in decreased scatter between analyses, resulting in a slight apparent improvement in precision, as shown by the $2 s$ values of the analyses (Figure 4, Table 1). This subtle difference observed between the two methods may be attributable to small changes in instrumental mass bias within the analysis, which are not effectively accounted for by the means-of-ratios method.

This article is protected by copyright. All rights reserved. 


\section{Estimation of measurement repeatability}

Previous DS studies have estimated measurement uncertainties on DS-corrected ratios using Monte Carlo simulations (Fantle and Bullen 2009, John 2012), linear uncertainty propagation (Hamelin et al. 1985, Rudge et al. 2009) or by a least-squares approach (Powell et al. 1998). These uncertainties are generated from theoretical considerations and modelled probability distributions for the inputs to the DS equations, and take into account factors such as amplifier noise and counting statistics. However, those methods typically do not take the variance in measured ratios as an input, and the estimated measurement repeatability are essentially invariant for a given DS mixture.

Since IsoSpike calculates DS-corrected ratios for every time slice within an analysis, calculation of statistics to be performed directly on calculated results providing a direct assessment of internal errors (i.e., the 2SE of DS-corrected ratios). As these are based on real measurements they inherently incorporate all sources of measurement uncertainty. As with other methods, IsoSpike does not include uncertainty in the DS composition or the reference material composition, though these are considered to be fixed and do not change during the course of a mass spectrometer run (e.g., Rudge et al. 2009). Variations in measured ratios are propagated through to DS-corrected ratios, and the 2SE of an analysis provides a realistic assessment of the quality of the analysis. As the time interval for each analysis that is used in calculations can be adjusted in lolite, it is possible for the analyst to adjust the time interval to see how the resultant uncertainty on the DS-corrected result changes due to counting statistics. This allows the ideal sample measurement time for a given required level of precision to be determined. Additionally, by allowing the analyst to see each result, outliers may be manually identified and removed where justified. Alternatively, lolite removes outliers from the final result based on points that are greater than 3 s or $2 s$ from the mean. Similarly, no outlier rejection may also be selected.

Use of splines

In stable isotope studies, natural isotope fractionations are usually expressed as a relative difference in some ratio between the sample and a reference material, as for example in $\delta$ notation (i.e., Equation 2). It is common practice to make measurements of the RM during a mass spectrometer session against which measurements of unknowns are calibrated. In spreadsheet data reduction routines, this is generally done by either taking the average composition for the RM measured during the session, or by interpolating between measurements. Iolite uses splines to interpolate RM and baseline compositions between measurements, and a number of different spline fitting modes are available (e.g., averages,

This article is protected by copyright. All rights reserved. 
linear interpolation, smoothed cubic fitting), and their features are discussed in detail in Paton et al. (2011). Splines that use averages or linear interpolations are equivalent to the methods typically employed in spreadsheet data reduction, while the more sophisticated spline types may provide more accurate estimates of changing baseline or RM compositions over time.

The effect of using different spline types for DS data with IsoSpike were investigated by reprocessing data for the Pt reference solution of Baker (Figure 4 and Table 1) with some of the available spline types. The results are shown in Table 2. Combinations using an average_mean spline for reference materials resulted in an enlarged measurement uncertainty budget as they do not account for any drift in RM values. Other spline combinations can affect the precision by ca. $10 \%$. While the effect on this example data set is relatively minor, this might not be the case for all applications. For example, in applications where baseline corrections are larger, the choice of baseline interpolation may have a greater effect than that in the example presented here. Our aim here is simply to highlight that the choice of method of interpolation may have an impact on the final result. This choice lies with the analyst and should (ideally) represent an empirical best fit to processes occurring within the mass spectrometer. The interpolation method in lolite can easily be changed to examine this effect.

\section{Coherency and applicability}

One of the main advantages of the method presented here is the coherency of the data reduction and uncertainty estimation process. Reduction of DS data from a typical mass spectrometer session could be completed in less than one minute, from importing raw data to outputting finished DS-corrected results. This included a rigorous estimate of the internal precision that might otherwise be undertaken as a separate step or by applying a fixed precision to all analyses. The ability of Iolite to handle raw data files from a variety of mass spectrometers means that there is very little manual handling involved, removing the potential for operator error. At the same time, many functions within lolite are open source, including all data reduction schemes (DRS), statistical functions, and IsoSpike itself, providing complete transparency (and, if required, modification) of the data reduction process. IsoSpike is designed to be quickly and easily extended to new DS isotope systems, even by users with limited experience with lolite. The example files shown here are also available for download, and provide a template for those wishing to proceed further.

This article is protected by copyright. All rights reserved. 


\section{Conclusions}

The IsoSpike software for correction of DS data presented here provides a complete and intuitive data reduction process that can be applied to any DS system and is freely available for use by others. Data reduction is straightforward, fast, and provides rigorous estimates of internal errors and possible improvements in external reproducibility. The integration-by-integration approach of IsoSpike provides, at least, a faster and more consistent approach, and possibly a more accurate way to process DS data than commonly employed methods. Combined with the capabilities built into lolite such as data visualisation and exporting, this combination has the potential to be useful to the double-spike community.

\section{Acknowledgements}

The authors wish to thank Thomas Nägler and an anonymous reviewer for their insightful and constructive reviews, and Thomas Meisel for his editorial handling. We are also grateful to Chad Paton for helpful discussions during the preparation of this paper and Joel Baker for constructive comments on a draft version of this manuscript.

\section{References}

Albarède F. and Beard B. (2004)

Analytical methods for non-traditional isotopes. Reviews in Mineralogy and Geochemistry, 55, 113-152.

Anbar A.D. (2004)

Molybdenum stable isotopes: Observations, interpretations and directions. Reviews in Mineralogy and Geochemistry, 55, 429-454.

Baker J., Peate D., Waight T. and Meyzen C. (2004).

$\mathrm{Pb}$ isotopic analysis of standards and samples using a ${ }^{207} \mathrm{~Pb}-204 \mathrm{~Pb}$ double spike and thallium to correct for mass bias with a double-focusing MC-ICP-MS. Chemical Geology, 211, 275-303.

Berglund M. and Wieser M.E. (2011)

Isotopic compositions of the elements 2009 (IUPAC technical report). Pure and Applied Chemistry, 83, 397410.

This article is protected by copyright. All rights reserved. 


\section{Compston W. and Oversby V.M. (1969)}

Lead isotopic analysis using a double spike. Journal of Geophysical Research, 74, 4338-4348.

Creech J., Baker J., Handler M., Schiller M. and Bizzarro M. (2013)

Platinum stable isotope ratio measurements by double-spike multiple collector ICP-MS. Journal of Analytical Atomic Spectrometry, 28, 853-865.

\section{Dodson M. (1970)}

Simplified equations for double-spiked isotopic analyses. Geochimica et Cosmochimica Acta, 34, 12411244.

\section{Dodson M.H. (1963)}

A theoretical study of the use of internal standards for precise isotopic analysis by the surface ionization technique: Part i - general first-order algebraic solutions. Journal of Scientific Instruments, 40, $289-295$.

Fantle M.S. and Bullen T.D. (2009)

Essentials of iron, chromium, and calcium isotope analysis of natural materials by thermal ionization mass spectrometry. Chemical Geology, 258, 50-64.

Galer S.J.G. (1999)

Optimal double and triple spiking for high precision lead isotopic measurement. Chemical Geology, 157, 255-274.

Hamelin B., Manhès G., Albarède F. and Allègre C.J. (1985)

Precise lead isotope measurements by the double spike technique: A reconsideration. Geochimica et Cosmochimica Acta, 49, 173-182.

Jenkins M.A. and Traub J.F. (1975)

Principles for testing polynomial zerofinding programs. ACM Transactions on Mathematical Software, 1, 2634.

John S.G. (2012)

Optimizing sample and spike concentrations for isotopic analysis by double-spike ICP-MS. Journal of Analytical Atomic Spectrometry, 27, 2123-2131.

\section{Johnson C.M. and Beard B.L. (1999)}

Correction of instrumentally produced mass fractionation during isotopic analysis of Fe by thermal ionization mass spectrometry. International Journal of Mass Spectrometry, 193, 87-99.

This article is protected by copyright. All rights reserved. 
Maréchal C.N., Télouk P. and Albarède F. (1999)

Precise analysis of copper and zinc isotopic compositions by plasma-source mass spectrometry. Chemical Geology, 156, 251-273.

Markey R., Hannah J.L., Morgan J.W. and Stein H.J. (2003)

A double spike for osmium analysis of highly radiogenic samples. Chemical Geology, 200, 395-406.

Paton C., Hellstrom J., Paul B., Woodhead J. and Hergt J. (2011)

Iolite: Freeware for the visualisation and processing of mass spectrometric data. Journal of Analytical

Atomic Spectrometry, 26, 2508-2518.

Powell R., Woodhead J. and Hergt J. (1998)

Uncertainties on lead isotope analyses: Deconvolution in the double-spike method. Chemical Geology, 148, 95-104.

Rudge J.F., Reynolds B.C. and Bourdon B. (2009)

The double spike toolbox. Chemical Geology, 265, 420-431.

Russell R.D. (1971)

The systematics of double spiking. Journal of Geophysical Research, 76, 4949-4955.

Siebert C., Nägler T.F. and Kramers J.D. (2001)

Determination of molybdenum isotope fractionation by double-spike multicollector inductively coupled plasma-mass spectrometry. Geochemistry Geophysics Geosystems, 2, 1032.

Wolff Briche C., Held A., Berglund M., De Bièvre P. and Taylor P. (2002)

Measurement of the isotopic composition and atomic weight of an isotopic reference material of platinum, IRMM-010. Analytica Chimica Acta, 460, 41-47.

\section{Woodhead J.D. and Hergt J.M. (1997)}

Application of the 'double spike' technique to Pb-isotope geochronology. Chemical Geology, 138, 31 1321.

Woodhead J. (2002)

A simple method for obtaining highly accurate $\mathrm{Pb}$ isotope data by MC-ICP-MS. Journal of Analytical Atomic Spectrometry, 17, 1381-1385.

This article is protected by copyright. All rights reserved. 


\section{Figure captions}

Figure 1. Automatic integration selection window in lolite. Text strings can be used to quickly select groups of samples.

Figure 2. IsoSpike options window where the user can select how the DS parameters are determined. Settings can be stored in a plain text file, which can be loaded from the options menu or created through a guided process.

Figure 3. Iolite report window with processed DS-corrected Pt stable isotope data. Error bars reflect the internal precision (2SE), which is based on the distribution of the results of the doublespike inversion from each measurement integration within each analysis.

Figure 4. DS-corrected Pt stable isotope data for IRMM-010 and the Baker Pt reference solution. DS correction has been performed both on mean ratios for each analysis and by integration-byintegration correction of every integration. Error bars reflect either estimated 2SE uncertainties determined using the linear error propagation function in the Double Spike Toolbox (Rudge et al. 2009) for mean ratios data, or observed 2SE uncertainties for integration-by-integration corrected data.

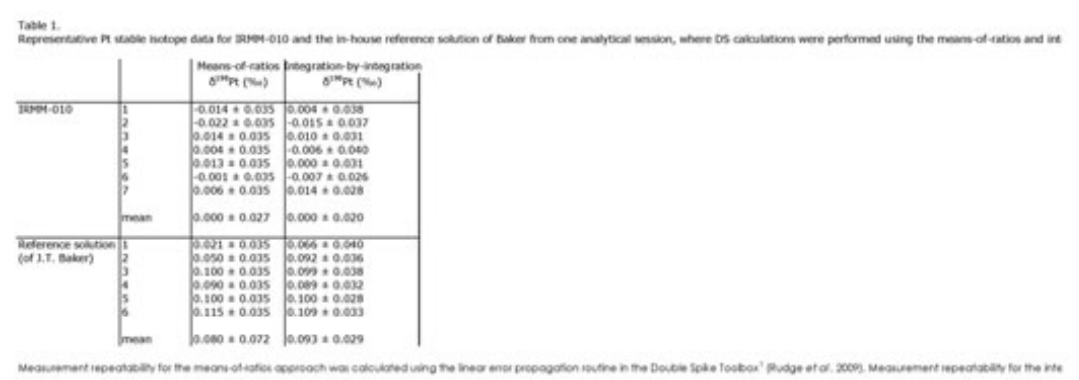

This article is protected by copyright. All rights reserved. 
Table 2.

Double-spike-corrected Pt stable isotope results for the reference solution of Baker using different spline types for the IRMM referenc

\begin{tabular}{|c|c|c|}
\hline \multicolumn{2}{|c|}{ Spline type } & \multirow{2}{*}{$\begin{array}{l}\text { Baker Pt refere } \\
8^{190} \mathrm{Pt}(\%)=\end{array}$} \\
\hline Baseline & RM & \\
\hline Average_mean & Average_mean & $0.090 \pm 0.051$ \\
\hline Linearfit & Linearfit & $0.093 \pm 0.032$ \\
\hline LinearInterpolation & LinearInterpolation & $0.098 \pm 0.034$ \\
\hline StepForward & Average_mean & $0.087 \pm 0.043$ \\
\hline StepForward & Linearfit & $0.090 \pm 0.030$ \\
\hline StepForward & Linearinterpolation & $0.087 \pm 0.034$ \\
\hline Stepforward & SmoothAuto & $0.090=0.030$ \\
\hline Smooth_auto & Average_mean & $0.089 \pm 0.044$ \\
\hline Smooth_auto & Unearfit & $0.092 \pm 0.031$ \\
\hline Smooth_auto & LinearInterpolation & $0.089 \pm 0.035$ \\
\hline Smooth_auto & SmoothAuto & $0.092 \pm 0.031$ \\
\hline LinearInterpolat & Average_mean & $0.088 \div 0.044$ \\
\hline LinearInterpolat & Linearfit & $0.091 \pm 0.030$ \\
\hline LinearInterpolation & LinearInterpolation & $0.088 \pm 0.034$ \\
\hline LinearInterpolation & SmoothAuto & $0.091 \pm 0.030$ \\
\hline
\end{tabular}

Uncertainties are $2 s$ of six analyses of the reference solution of Baker.

This article is protected by copyright. All rights reserved. 


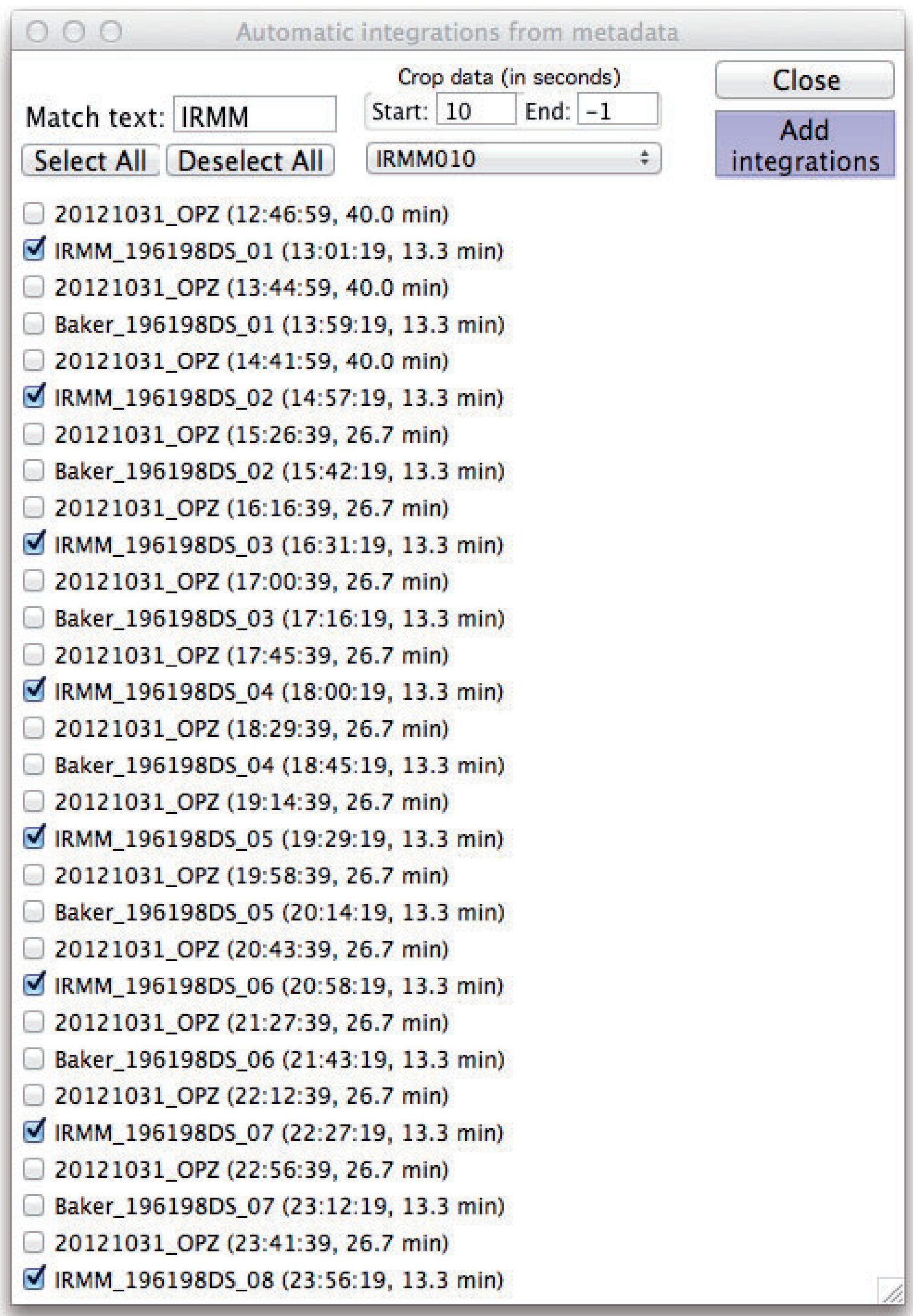

This article is protected by copyright. All rights reserved. 


\section{Double Spike Options}

Get DS_settings from:

Use current DS settings file

Load DS_settings file

Current DS settings file:

DS_Settings_290ct2012_VUW-IRMM_196198DS.txt

\}$\square$ Review DS parameters

\section{Create DS_settings file}

\section{Cancel}

\begin{tabular}{|c|c|c|c|c|c|c|c|}
\hline & \multicolumn{4}{|c|}{ Calculate statistics for the channel: Deltalnt198_194 } & \multicolumn{3}{|c|}{$\begin{array}{c}\text { Stats methods used } \\
\text { Baselines : Mean with } 2 \text { S.D. outlier reject } \\
\text { Normal integrations : Mean with } 2 \text { S.D. outlier reject }\end{array}$} \\
\hline & Integration Name & $\begin{array}{l}\text { Outlier rejection } \\
\text { None }\end{array}$ & $\begin{array}{l}\text { Average } \\
\text { mean }\end{array}$ & $\begin{array}{l}\text { Uncertainty } \\
2 \text { S.D. (absolute) }\end{array}$ & MSWD & Prob. of fit & $\begin{array}{l}\text { Propagated } \\
\text { uncertainties }\end{array}$ \\
\hline & Baseline_1 & 0 (of 0) & 0 & no value & $<5$ valid & $s<5$ valid valu & SNo \\
\hline \multirow{2}{*}{$\begin{array}{l}\text { Page } 1 \\
\text { (of } 1 \text { ) }\end{array}$} & Baker & 0 (of 6) & 0.092 & 0.030 & 0.65 & 0.66 & No \\
\hline & IRMM010 & 0 (of 8) & 0.001 & 0.027 & 0.55 & 0.8 & No \\
\hline
\end{tabular}

Baker $=0.092 \pm 0.030$

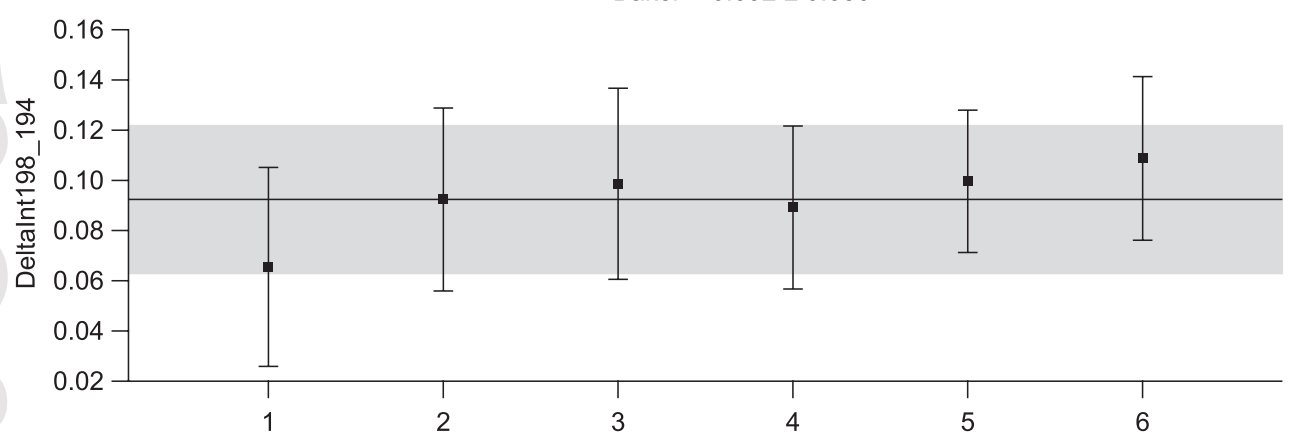

Plot options

Population statistics Average, "mean" Uncertainty, "2 S.D. (absolute)"

Sample statistics Internal (2 S.E.)

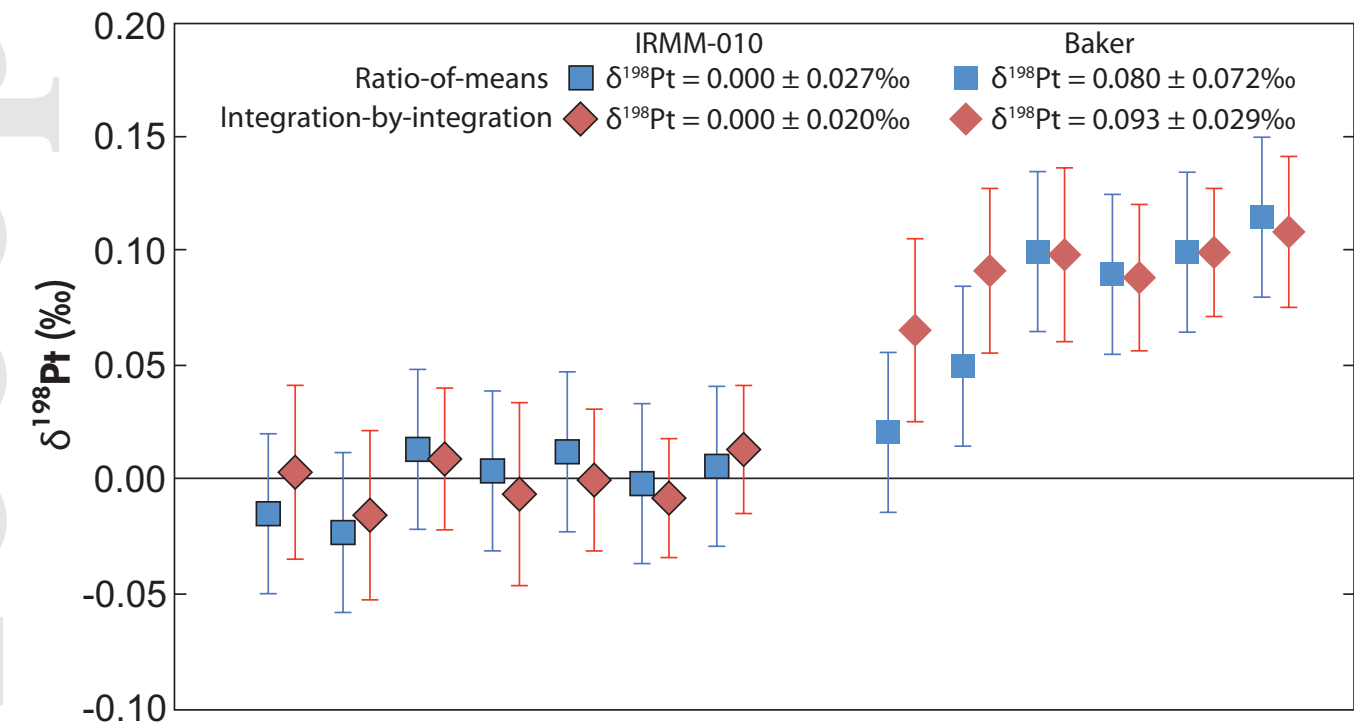

This article is protected by copyright. All rights reserved. 